\title{
Synthesis of Novel Heterocycles by Amide Activation and Umpolung Cyclization
}

Haoqi Zhang, ${ }^{+}$Margaux Riomet, ${ }^{+}$Alexander Roller and Nuno Maulide*

Institute of Organic Chemistry, Faculty of Chemistry, University of Vienna, Währinger Straße 38, 1090 Vienna, Austria.

Institute of Inorganic Chemistry, Faculty of Chemistry, University of Vienna, Währinger Straße 42, 1090 Vienna, Austria. 


\section{Contents}

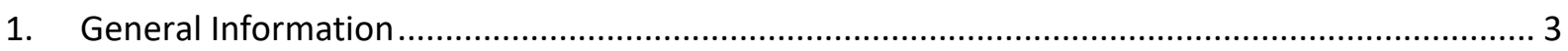

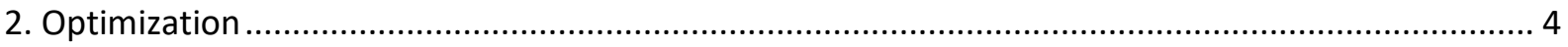

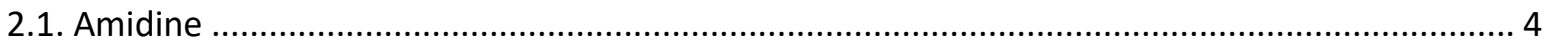

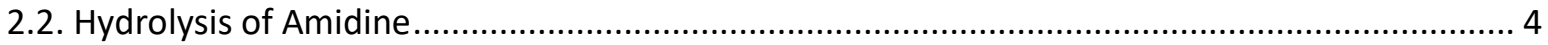

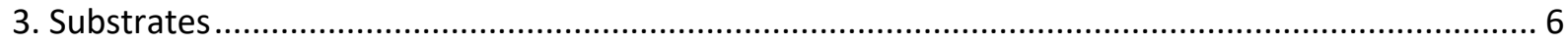

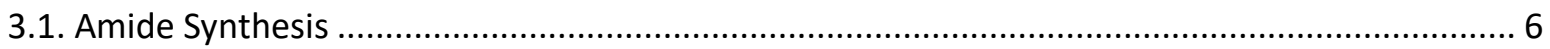

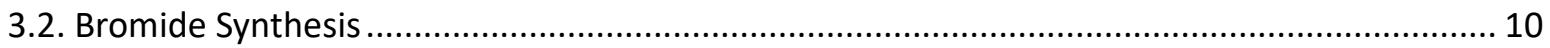

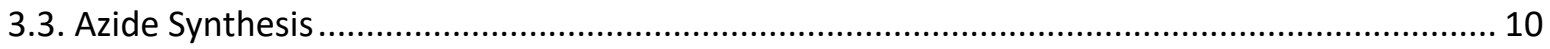

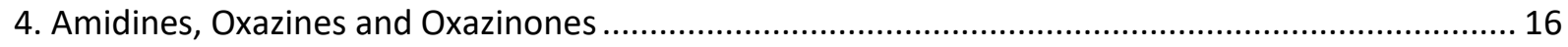

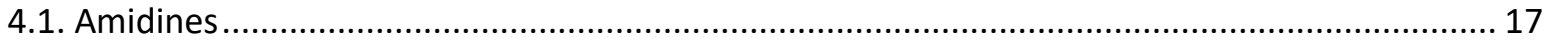

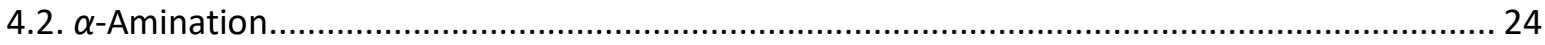

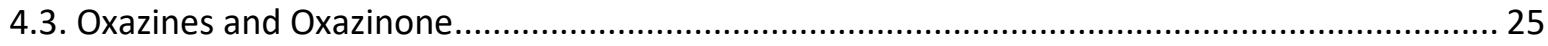

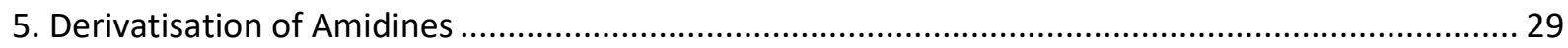

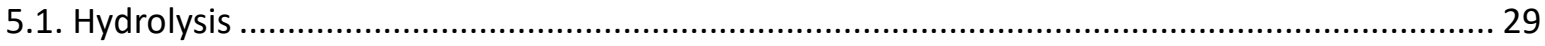

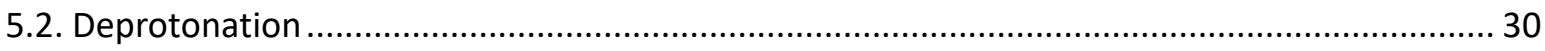

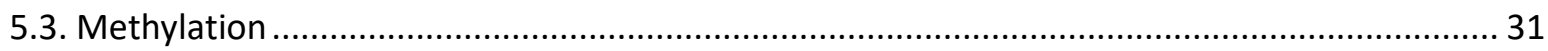

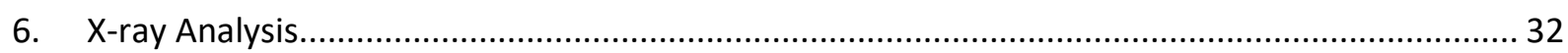

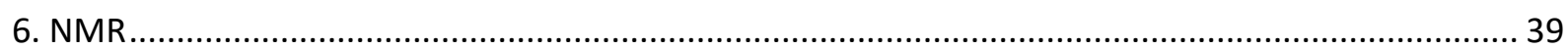




\section{General Information}

Unless otherwise stated, all glassware was flame-dried before use and all reactions were performed under an atmosphere of argon with anhydrous solvents. Triflic anhydride was distilled over $\mathrm{P}_{2} \mathrm{O}_{5}$ prior to use. All other reagents were used as received from commercial suppliers unless otherwise stated. Reaction requiring higher temperature were heated in an oil bath. Reaction progress was monitored by thin layer chromatography (TLC) performed on aluminium plates coated with silica gel F254 with $0.2 \mathrm{~mm}$ thickness. Chromatograms were visualized by fluorescence quenching with UV light at $254 \mathrm{~nm}$ or by staining using potassium permanganate. Flash column chromatography was performed using silica gel 60 (230-400 mesh, Merck and co.). DMA mixture for column chromatography was made of DCM, MeOH, concentrated ammonia (90:10:1). Neat infra-red spectra were recorded using a PerkinElmer Spectrum 100 FT-IR spectrometer. Wavenumbers (vmax) are reported in cm-1. Mass spectra were obtained using a Finnigan MAT 8200 or $(70 \mathrm{eV})$ or an Agilent $5973(70 \mathrm{eV})$ spectrometer, using electrospray ionization (ESI). All $1 \mathrm{H}$ NMR and $13 \mathrm{C}$ NMR spectra were recorded using a Bruker AV-400, AV-600 and AV-700 spectrometer at 300K. Chemical shifts were given in parts per million (ppm, $\delta$ ) and coupling constants $(J)$ are quoted in $\mathrm{Hz}$, referenced to the solvent peak of $\mathrm{CDCl} 3$, defined at $\delta=7.26$ ppm (1H NMR) and $\delta=77.16$ (13C NMR). Coupling constants are quoted in $\mathrm{Hz}(\mathrm{J})$. $1 \mathrm{H} \mathrm{NMR}$ splitting patterns were designated as singlet ( $(\mathrm{s})$, doublet (d), triplet ( $t)$, quartet (q), pentet ( $p)$. Splitting patterns that could not be interpreted or easily visualized were designated as multiplet $(\mathrm{m})$ or broad (br). Selected 13C NMR spectra were recorded using the attached proton test (APT) to facilitate the confirmation and assignment of the structure. 


\section{Optimization}

\subsection{Amidine}

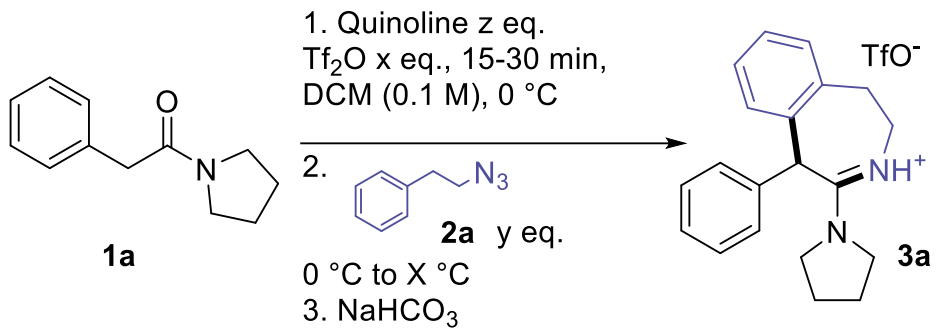

\begin{tabular}{|c|c|c|c|c|c|c|c|}
\hline Entry & $x(e q)$ & $t(\min )$ & y (eq) & Quinoline & $z$ (eq) & $\mathbf{T}$ & Yield \\
\hline 1 & 2 & 15 & 2 & & 5 & $\mathrm{rt}$ & $48 \%$ \\
\hline 2 & 2 & 15 & 2 & & 5 & $\mathrm{rt}$ & - \\
\hline 3 & 2 & 15 & 2 & & 5 & $\mathrm{rt}$ & - \\
\hline 4 & 2 & 15 & 2 & & 2 & $\mathrm{rt}$ & $63 \%$ \\
\hline 5 & 2 & 15 & 2 & & 2 & $\mathrm{rt}$ & $75 \%$ \\
\hline 6 & 2 & 15 & 2 & & 2 & $40^{\circ} \mathrm{C}$ & $63 \%$ \\
\hline 7 & 2 & 15 & 2 & & 2 & $\mathrm{rt}$ & $66 \%$ \\
\hline 8 & 2 & 15 & 1.1 & & 2 & $\mathrm{rt}$ & $78 \%$ \\
\hline 9 & 1.1 & 15 & 1.1 & & 2 & $\mathrm{rt}$ & $53 \%$ \\
\hline 10 & 1.1 & 30 & 1.1 & & 2 & $\mathrm{rt}$ & $59 \%$ \\
\hline 11 & 1.4 & 15 & 1.1 & & 2 & $\mathrm{rt}$ & $67 \%$ \\
\hline
\end{tabular}

Table S1 Reaction optimization. Yields refer to isolated product. Reactions were carried out on $0.2 \mathrm{mmol}$ scale.

\subsection{Hydrolysis of Amidine}

To a solution of $\mathbf{3 a}$ in [solvent] was added aqueous [base]. The mixture was stirred for [time] under reflux. After cooling down and quenching with $\mathrm{NH}_{4} \mathrm{Cl}$ (sat.), the layers were separated and the aqueous phase was washed with $\mathrm{DCM}(3 \mathrm{x})$. The combined organic layers were dried over $\mathrm{Na}_{2} \mathrm{SO}_{4}$ and 
concentrated under reduced pressure. The product was purified via column chromatography (silica, DMA-DCM gradient, 0 to $20 \%$ ).<smiles>c1ccc(C2C(N3CCCC3)=NCCc3ccccc32)cc1</smiles>

3a

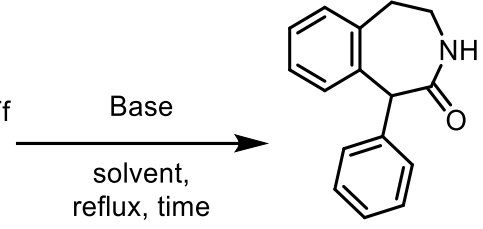

$6 \mathbf{a}$

\begin{tabular}{ccccc} 
Entry & Base & Solvent & Time & Yield $^{\text {a }}$ \\
\hline 1 & $1.0 \mathrm{~mL} \mathrm{NaOH}(1 \mathrm{M}, 5 \mathrm{eq})$ & $1.0 \mathrm{~mL} \mathrm{THF}$ & $15 \mathrm{~h}$ & $56 \%$ \\
2 & $2.0 \mathrm{~mL} \mathrm{NaOH}(1 \mathrm{M}, 10 \mathrm{eq})$ & $2.0 \mathrm{~mL} \mathrm{THF}$ & $24 \mathrm{~h}$ & $43 \%$ \\
3 & $1.0 \mathrm{~mL} \mathrm{NaOH}(5 \mathrm{M}, 25 \mathrm{eq})$ & $1.0 \mathrm{~mL} \mathrm{THF}$ & $24 \mathrm{~h}$ & $5 \%$ \\
4 & $1.0 \mathrm{~mL} \mathrm{NaOH}(1 \mathrm{M}, 5 \mathrm{eq})$ & $1.0 \mathrm{~mL} \mathrm{EtOH}$ & $15 \mathrm{~h}$ & $7 \%$ \\
5 & $1.0 \mathrm{~mL} \mathrm{KOH}(1 \mathrm{M}, 5 \mathrm{eq})$ & $1.0 \mathrm{~mL} \mathrm{THF}$ & $15 \mathrm{~h}$ & $10 \%$ \\
6 & $1.0 \mathrm{~mL} \mathrm{NaOH}(1 \mathrm{M}, 5$ eq) & $1.0 \mathrm{~mL} \mathrm{Dioxane}$ & $15 \mathrm{~h}$ & $22 \%$ \\
7 & $1.0 \mathrm{~mL} \mathrm{NaOH}(1 \mathrm{M}, 5$ eq) & $5.0 \mathrm{~mL} \mathrm{THF}$ & $15 \mathrm{~h}$ & $0 \%$ \\
8 & $1.0 \mathrm{~mL} \mathrm{NaOH}(1 \mathrm{M}, 5 \mathrm{eq})$ & $1.0 \mathrm{~mL} \mathrm{THF}$ & $15 \mathrm{~h}$ & $63 \%$
\end{tabular}

Table S2 Hydrolysis optimization. Yields refer to isolated product. Reactions were carried out on $0.2 \mathrm{mmol}$ scale.

Reaction conditions: A mixture of $3 a(0.2 \mathrm{mmol}, 1$ eq.), solvent and base were stirred under reflux for $15 \mathrm{~h}$. Isolated yields. 


\section{Substrates}

\subsection{Amide Synthesis}

\section{General Procedure A}

To a solution of the amine (1.0 eq.) and triethylamine $\left(2.0\right.$ eq.) in $\mathrm{DCM}(0.1 \mathrm{M})$ at $0{ }^{\circ} \mathrm{C}$, the corresponding acyl chloride (1.2 eq.) was added dropwise and the resulting reaction mixture was allowed to warm up to room temperature while stirring for $14 \mathrm{~h}$. Afterwards, the reaction was quenched with a saturated aqueous solution of sodium bicarbonate and the biphasic system was separated. After extraction of the aqueous phase with DCM $(2 x)$, the organic phases were combined and dried over anhydrous sodium sulfate. The dried solution was filtered and concentrated under reduced pressure. The resulting crude material was purified by flash column chromatography on silica gel (heptane/ethyl acetate, 40:60) to afford the desired compound.

\section{General Procedure B}

The acyl chloride was first prepared via addition of oxalyl chloride ( 3 eq.) to a mixture of carboxylic acid (1 eq.) and catalytic amount of $\mathrm{N}, \mathrm{N}$-dimethylformamide $(0.1$ eq.) in DCM $(0.2 \mathrm{M})$ at room temperature. After stirring for $14 \mathrm{~h}$, the solvent and excess of oxalyl chloride were removed under reduced pressure. The crude acyl chloride was directly dissolved in DCM (0.2 M) and pyrrolidine (5 eq.) was added. The mixture was stirred for $5 \mathrm{~h}$ at room temperature. The reaction was quenched with a saturated aqueous solution of sodium bicarbonate. After separation, the organic layer was washed with $\mathrm{HCl}(1 \mathrm{M}), \mathrm{NaHCO}_{3}$ (sat.) and brine. The product was dried over anhydrous sodium sulfate and the solvent was removed under reduced pressure. The pure product was used without further purification.

\section{General Procedure C}

To a solution of carboxylic acid (1.0 eq.), HOBt (1.0 eq.) and EDCl (1.0 eq.) in DCM (0.5 M) were added the secondary amine (1.0 eq.) and triethylamine (1.0 eq.). The mixture was stirred for $16 \mathrm{~h}$ under argon atmosphere at room temperature. The mixture was diluted with EtOAc (5 DCM volumes). The organic layer was washed with $\mathrm{HCl} 1 \mathrm{M}$. then a saturated solution of $\mathrm{NaHCO}_{3}$ then brine. The organic layer was dried over $\mathrm{MgSO}_{4}$, filtered and evaporated under reduced pressure.

\section{General Procedure D}

HATU (1.2 eq.) was added to a solution of secondary amine (1.2 eq.), carboxylic acid (1 eq.) and triethylamine (2.4 eq.) in 0.4 M DMF. The reaction was stirred overnight at room temperature before quenching with $1 \mathrm{M} \mathrm{NaOH}$. The mixture was then extracted with ether, washed with sat. $\mathrm{NH}_{4} \mathrm{Cl}$ solution, dried over $\mathrm{Na}_{2} \mathrm{SO}_{4}$, and then evaporated. 


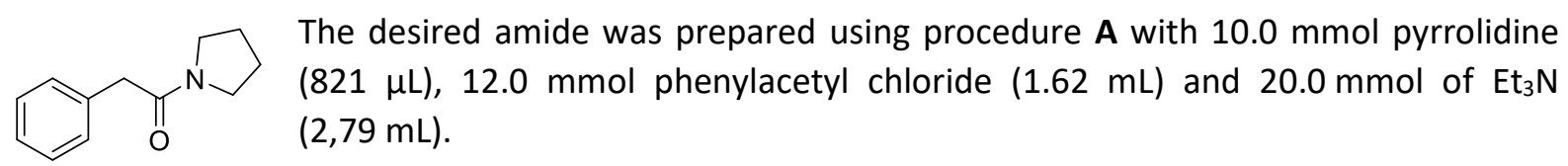

Yield (yellow-white solid): $91 \%$ (1.72 g).

Spectroscopic data are in agreement with the literature. ${ }^{1}$

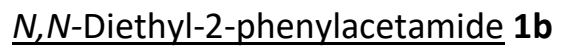<smiles>CCN(CC)C(=O)Cc1ccccc1</smiles>

The desired amide was prepared using procedure $\mathbf{C}$ with $7.34 \mathrm{mmol}$ diethylamine $(760 \mu \mathrm{L}), 7.34 \mathrm{mmol}$ phenylacetic acid $(1.00 \mathrm{~g}), 7.34 \mathrm{mmol}$ HOBt $(992 \mathrm{mg})$, $7.34 \mathrm{mmol} \mathrm{EDCl} . \mathrm{HCl}(1.41 \mathrm{~g})$ and $7.34 \mathrm{mmol}$ of $\mathrm{Et}_{3} \mathrm{~N}(1.02 \mathrm{~mL})$. The crude product was purified by column chromatography (Heptane /EtOAc, from $80 \% / 20 \%$ to

$0 \% / 100 \%$.$) .$

Yield (colourless oil): $82 \%$ (1.15 g).

Spectroscopic data are in agreement with the literature. ${ }^{2}$

1-(azetidin-1-yl)-2-phenylethan-1-one 1c

The desired amide was prepared using procedure $\mathrm{C}$ with $10 \mathrm{mmol}$ azetidine
hydrochloride $(936 \mu \mathrm{L}), 10 \mathrm{mmol}$ phenylacetic acid $(1.36 \mathrm{~g}), 10 \mathrm{mmol}$ HOBt $(1.35 \mathrm{~g})$,
$10 \mathrm{mmol} \operatorname{EDCl} . \mathrm{HCl}(1.92 \mathrm{~g})$ and $20 \mathrm{mmol}$ of $\mathrm{Et}_{3} \mathrm{~N}(2.79 \mathrm{~mL})$. The crude product was purified by column chromatography (Heptane /EtOAc, from $80 \% / 20 \%$ to $0 \% / 100 \%$.).

Yield (colourless oil): $29 \%$ (505 mg).

${ }^{1} \mathrm{H}$ NMR $\left(400 \mathrm{MHz}, \mathrm{CDCl}_{3}\right): \delta(\mathrm{ppm}) 7.35-7.21(\mathrm{~m}, 5 \mathrm{H}), 4.14-4.08(\mathrm{~m}, 2 \mathrm{H}), 4.06-4.00(\mathrm{~m}, 2 \mathrm{H}), 3.45(\mathrm{~s}, 2 \mathrm{H})$, $2.28-2.17(\mathrm{~m}, 2 \mathrm{H})$.

${ }^{13} \mathrm{C}$ NMR $\left(151 \mathrm{MHz}, \mathrm{CDCl}_{3}\right): \delta$ (ppm) 170.8, 134.7, 129.0 (2C), 128.6 (2C), 126.8, 50.6, 48.1, 39.1, 15.1.

HRMS (ESI) $\mathrm{m} / \mathrm{z}$ calculated for $[\mathrm{M}+\mathrm{H}]^{+} \mathrm{C}_{11} \mathrm{H}_{14} \mathrm{NO}^{+}: 176.1070$, found 176.1073 .

ATR-FTIR $\left(\mathrm{cm}^{-1}\right): 2951,1638,1424,1298,1118,720,695$.

\footnotetext{
${ }^{1}$ Pintori, D. G.; Greaney, M. F. Org. Lett. 2011, 13, 5713-5715.

2 Bannwart, L.; Abele, S.; Tortoioli, S. Synthesis 2016, 48, 2069-2078.
} 


\section{2-(2-bromophenyl)-1-(pyrrolidin-1-yl)ethan-1-one 1d}<smiles>O=C(Cc1ccccc1Br)N1CCCC1</smiles>

The desired amide was prepared using procedure $\mathbf{C}$ with $3.06 \mathrm{mmol}$ pyrrolidine (251 $\mu \mathrm{L}$ ), $3.06 \mathrm{mmol}$ 2-bromophenylacetic acid $(658 \mathrm{mg}), 3.06 \mathrm{mmol} \mathrm{HOBt}(468 \mathrm{mg})$, $3.06 \mathrm{mmol} \mathrm{EDCl} . \mathrm{HCl}(586 \mathrm{mg})$ and $3.06 \mathrm{mmol}$ of $\mathrm{Et}_{3} \mathrm{~N}(426 \mu \mathrm{L})$. The crude product was purified by column chromatography (Heptane /EtOAc, from $80 \% / 20 \%$ to $0 \% / 100 \%$.).

Yield (colourless oil): $97 \%$ (780 mg).

${ }^{1} \mathrm{H}$ NMR $\left(400 \mathrm{MHz}, \mathrm{CDCl}_{3}\right): \delta(\mathrm{ppm}) 7.55(\mathrm{~d}, J=8.0 \mathrm{~Hz}, 1 \mathrm{H}), 7.33(\mathrm{~d}, J=7.6 \mathrm{~Hz}, 1 \mathrm{H}), 7.27(\mathrm{dd}, J=10.8,3.4 \mathrm{~Hz}, 1 \mathrm{H})$, $7.13-7.09(\mathrm{~m}, 1 \mathrm{H}), 3.77(\mathrm{~s}, 2 \mathrm{H}), 3.52(\mathrm{t}, J=6.9 \mathrm{~Hz}, 2 \mathrm{H}), 3.48(\mathrm{t}, J=6.8 \mathrm{~Hz}, 2 \mathrm{H}), 1.96(\mathrm{~m}, 2 \mathrm{H}), 1.87(\mathrm{~m}, 2 \mathrm{H})$.

${ }^{13} \mathrm{C}$ NMR (151 MHz, $\left.\mathrm{CDCl}_{3}\right): \delta$ (ppm) 168.6, 135.4, 132.7, 131.1, 128.6, 127.7, 125.0, 47.0, 46.1, 42.2, 26.3, 24.6.

HRMS (ESI) $\mathrm{m} / \mathrm{z}$ calculated for $[\mathrm{M}+\mathrm{Na}]^{+} \mathrm{C}_{12} \mathrm{H}_{14} \mathrm{BrNNaO}^{+}: 290.0151$, found 290.0149 .

ATR-FTIR $\left(\mathrm{cm}^{-1}\right)$ : 2951, 2880, 1638, 1424, 1118, 720, 695.

\section{2-(4-methoxyphenyl)-1-(pyrrolidin-1-yl)ethan-1-one 1e}<smiles>COc1ccc(CC(=O)N2CCCC2)cc1</smiles>

The desired amide was prepared using procedure B with $1.0 \mathrm{mmol}$ 4Methoxyphenylacetic Acid (166 mg), $0.10 \mathrm{mmol}$ DMF (7.7 $\mu \mathrm{L}), 3.0 \mathrm{mmol}$ oxalyl chloride $(254 \mu \mathrm{L})$ and $5.0 \mathrm{mmol}$ pyrrolidine $(411 \mu \mathrm{L})$.

Yield (yellow oil): 95 \% (209 mg).

Spectroscopic data are in agreement with the literature. ${ }^{3}$

\section{$\underline{\text { 2-(4-nitrophenyl)-1-(pyrrolidin-1-yl)ethan-1-one } \mathbf{1 f}}$}<smiles>O=C(Cc1ccc([N+](=O)[O-])cc1)N1CCCC1</smiles>

The product was obtained using general procedure $\mathbf{D}$ from $5.0 \mathrm{mmol} 4$ nitrophenylacetic acid ( $906 \mathrm{mg}$ ), $6.0 \mathrm{mmol}$ pyrrolidine $(493 \mu \mathrm{L}), 6.0 \mathrm{mmol}$ HATU $(2.28 \mathrm{~g})$ and $12 \mathrm{mmol}$ triethylamine $(1.67 \mathrm{~mL})$. The crude product was purified by column chromatography (Heptane /EtOAc, from $80 \% / 20 \%$ to $0 \% / 100 \%$.).

Yield (yellow oil): $81 \%$ (945 mg)

Spectroscopic data are in agreement with the literature. ${ }^{4}$

\footnotetext{
${ }^{3}$ Moeller, K. D.; Wang, P. W.; Tarazi, S.; Marzabadi, M. R.; Wong, P. L. J. Org. Chem. 1991, 56, 1058-1067.

${ }^{4}$ Huh, D. H.; Jeong, J. S.; Lee, H. B.; Ryu, H.; Kim, Y. G. Tetrahedron 2002, 58, 9925-9932.
} 


\section{1-(pyrrolidin-1-yl)-2-(4-(trifluoromethyl)phenyl)ethan-1-one $\mathbf{1 g}$}<smiles>O=C(Cc1ccc(C(F)(F)F)cc1)N1CCCC1</smiles>

The desired amide was prepared using modified procedure $\mathbf{C}$ with THF as solvent and $3.76 \mathrm{mmol}$ pyrrolidine $(309 \mu \mathrm{L}), 2.89 \mathrm{mmol}$ 4-trifluorophenylacetic acid (590 mg), $3.76 \mathrm{mmol} \mathrm{HOBt} \mathrm{(508} \mathrm{mg),} 3.76 \mathrm{mmol} \mathrm{EDCl} . \mathrm{HCl}(720 \mathrm{mg}$ ) and $7.51 \mathrm{mmol}$ of $\mathrm{Et}_{3} \mathrm{~N}(1.05 \mathrm{~mL})$. The crude product was purified by column chromatography (Heptane /EtOAc, from $80 \% / 20 \%$ to $0 \% / 100 \%$.).

Yield (white solid): $88 \%$ (653 mg).

Spectroscopic data are in agreement with the literature. ${ }^{4}$

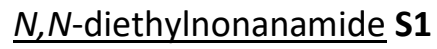<smiles>CCCCCCCCC(=O)N(CC)CC</smiles>

The desired amide was prepared using procedure $A$ with $6.0 \mathrm{mmol}$ diethylamine $(621 \mu \mathrm{L}), 5.0 \mathrm{mmol}$ nonanoyl chloride $(901 \mu \mathrm{L})$ and $10.0 \mathrm{mmol}$ of $\mathrm{Et}_{3} \mathrm{~N}(1.39 \mathrm{~mL})$.

Yield (yellow oil): quant. (1.01 g).

Spectroscopic data are in agreement with the literature. ${ }^{5}$

\section{$\underline{N}, N$-dimethyl-4-phenylbutanamide $\mathbf{S 2}$}<smiles>CN(C)C(=O)CCCc1ccccc1</smiles>

The product was prepared according to general procedure $\mathrm{C}$ from $2.46 \mathrm{~g}$ 4phenylbutyric acid and 4-nitrobenzenesulfonyl chloride $(15.0 \mathrm{mmol})$ and $7.5 \mathrm{~mL}$ dimethylamine (2.0 M in THF), (15.0 mmol). The crude product was purified by column chromatography (Heptane /EtOAc, from $80 \% / 20 \%$ to $0 \% / 100 \%$.).

Yield (yellow oil): $71 \%$ (2.04 g). in 71\% (2.04 g) yield.

Spectroscopic data are in agreement with the literature. ${ }^{6}$

\footnotetext{
${ }^{5}$ Fukuyama, T.; Nishitani, S.; Inouye, T.; Morimoto, K.; Ryu, I. Org. Lett. 2006, 8, 1383-1386.

${ }^{6}$ Zhou, X.; Zhang, G.; Gao, B.; Huang, Org. Lett. 2018, 20, 2208-2212.
} 


\subsection{Bromide Synthesis}

\section{2-bromo-1-(4-nitrophenyl)ethan-1-one S3}<smiles>O=C(CBr)c1ccc([N+](=O)[O-])cc1</smiles>

A solution of $6 \mathrm{mmol}$ bromine $(307 \mu \mathrm{L})$ in chloroform $(2 \mathrm{~mL})$ was slowly added to a solution of $5 \mathrm{mmol}$ 4-nitroacetophenone $(826 \mathrm{mg})$ in chloroform $(5 \mathrm{~mL})$ at $0^{\circ} \mathrm{C}$ under continuous stirring. The temperature of the reaction mixture was maintained at $0-5{ }^{\circ} \mathrm{C}$ during the addition. After stirring for $14 \mathrm{~h}$ at r.t., the solvent was removed under reduced pressure. The product was purified by recrystalization in EtOH (ca. $10 \mathrm{~mL}$ ) to yield pure compound.

Yield (yellow-white solid): $32 \%$ (386 mg).

Spectroscopic data are in agreement with the literature. ${ }^{7}$

\subsection{Azide Synthesis}

\section{General Procedure $\mathbf{E}$}

A solution of bromide (1 eq.) and $\mathrm{NaN}_{3}\left(1.5\right.$ eq.) in $\mathrm{DMF}(0.2 \mathrm{M})$ was stirred at $80{ }^{\circ} \mathrm{C}$ overnight. After $14 \mathrm{~h}$, the reaction mixture was cooled to r.t., diluted with $\mathrm{Et}_{2} \mathrm{O}$ or EtOAc. The biphasic system was separated and the organic layer was washed with ice-cooled $\mathrm{H}_{2} \mathrm{O}(4 \mathrm{x})$ and brine $(1 \mathrm{x})$. The pure product was obtained after drying over anhydrous $\mathrm{Na}_{2} \mathrm{SO}_{4}$ and evaporation of the solvent under reduced pressure to obtain the pure product which was used without further purifications.

\section{General Procedure F}

The corresponding halide was added to a solution of $\mathrm{NaN}_{3}$ (1.5 eq. or 3 eq.) in DMSO $(0.5 \mathrm{M})$ at $0{ }^{\circ} \mathrm{C}$ and the reaction mixture was stirred at $23{ }^{\circ} \mathrm{C}$ for $10 \mathrm{~min}$ or $14 \mathrm{~h}$. Ice-cooled $\mathrm{H}_{2} \mathrm{O}$ was added to the mixture and the mixture was extracted with diethyl ether $(3 \mathrm{x})$. The combined organic layers were washed with ice-cooled $\mathrm{H}_{2} \mathrm{O}(4 \mathrm{x})$ and brine (1x), dried over anhydrous $\mathrm{Na}_{2} \mathrm{SO}_{4}$ and the solvent was removed under reduced pressure to obtain the pure product which was used without further purifications.

\section{General Procedure G}

The corresponding chloride was added to a suspension of $\mathrm{NaN}_{3}$ (1.5 eq.) in acetone $(0.2 \mathrm{M})$ and stirred for $36 \mathrm{~h}$. Afterwards, the mixture was filtered. The filtrate was evaporated under reduced pressure to obtain the pure product which was used without further purification.

\footnotetext{
${ }^{7}$ Tada, N.; Ban, K.; Hirashima, S.; Miura, T.; Itoh, A. Direct Synthesis of $\alpha$-Bromoketones from Alkylarenes by Aerobic Visible Light Photooxidation. Org. Biomol. Chem. 2010, 8, 4701-4704.
} 


\section{(2-azidoethyl)benzene $2 \mathrm{a}$}

$\mathrm{N}_{3}$ The product was prepared using procedure $\mathrm{E}$ with $10 \mathrm{mmol}$ (2-Bromoethyl)benzene $(1.37 \mathrm{~mL})$ and $15 \mathrm{mmol}$ sodium azide $(975 \mathrm{mg})$ in DMF.

Yield (yellow oil): $87 \%$ (1.29 g).

Spectroscopic data are in agreement with the literature. ${ }^{8}$

\section{1-(2-azidoethyl)-2-methylbenzene $\mathbf{2 b}$}<smiles>Cc1ccccc1CCN</smiles>

The product was prepared using procedure $\mathbf{E}$ with $1 \mathrm{mmol}$ 2-Methylphenethyl bromide $(169 \mu \mathrm{L})$ and $1.5 \mathrm{mmol}$ sodium azide $(97.5 \mathrm{mg})$ in DMF.

Yield (yellow oil): $93 \%$ (150 mg).

Spectroscopic data are in agreement with the literature. ${ }^{9}$

\section{1-(2-azidoethyl)-3-methoxybenzene $2 c$}

$\mathrm{MeO} \mathrm{N}_{3}$ The product was prepared using procedure $\mathbf{E}$ with $1 \mathrm{mmol}$ 3-methoxyphenethyl bromide $(215 \mathrm{mg})$ and $1.5 \mathrm{mmol}$ sodium azide $(97.5 \mathrm{mg})$ in DMF.

Yield (clear oil): 90 \% (160 mg).

Spectroscopic data are in agreement with the literature. ${ }^{9}$

${ }^{8}$ Kalkeren, H. A. van; Bruins, J. J.; Rutjes, F. P. J. T.; van Delft, F. L. Advanced Synthesis \& Catalysis 2012, 354, 1417-1421.

${ }^{9}$ Suzuki, T.; Ota, Y.; Ri, M.; Bando, M.; Gotoh, A.; Itoh, Y.; Tsumoto, H.; Tatum, P. R.; Mizukami, T.; Nakagawa, H.; et al. J. Med. Chem. 2012, 55, 9562-9575. 


\section{1-(2-azidoethyl)-4-fluorobenzene $\mathbf{2 d}$}<smiles>[NH-]CCc1ccc(F)cc1</smiles>

To a $0.5 \mathrm{M}$ solution of $\mathrm{NaN}_{3}$ (1.1 eq., $2.2 \mathrm{mmol}, 143 \mathrm{mg}$ ) in $4 \mathrm{~mL}$ DMSO was added p-Fluorophenethyl bromide (1.0 eq., $2 \mathrm{mmol}, 0.28 \mathrm{~mL}$ ), and the mixture was stirred at $80{ }^{\circ} \mathrm{C}$ and periodically monitored by TLC. When the reaction was completed, the mixture was quenched with water and stirred until it cooled down to room temperature and then extracted with $\mathrm{Et}_{2} \mathrm{O}$. The organic layer was separated, washed with water and brine, and dried over $\mathrm{Na}_{2} \mathrm{SO}_{4}$. Filtration, concentration in vacuo, and purification of the residue by silica gel flash column chromatography gave the corresponding alkyl azide.

Yield (clear oil): quantitative $(330 \mathrm{mg})$.

Spectroscopic data are in agreement with the literature. ${ }^{9}$

\section{1-(2-azidoethyl)-3-nitrobenzene 2e}

$\mathrm{O}_{2} \mathrm{~N} \mathrm{~N}_{3}$ The product was prepared using procedure $\mathbf{E}$ with $1 \mathrm{mmol}$ bromide $(230 \mathrm{mg})$ and $1.5 \mathrm{mmol}$ sodium azide $(97.5 \mathrm{mg})$ in DMF.

Yield (yellow oil): $99 \%$ (191 mg).

Spectroscopic data are in agreement with the literature. ${ }^{9}$

\section{Benzyl azide $\mathbf{2 f}$}

The product was obtained using general procedure $\mathbf{E}$ from $10 \mathrm{mmol}$ benzyl bromide N $(1.19 \mathrm{~mL})$ and $15 \mathrm{mmol}$ sodium azide $(975 \mathrm{mg})$.

Yield (yellow oil): quantitative (1.35 g).

Spectroscopic data are in agreement with the literature. ${ }^{10}$

\section{1-(azidomethyl)-3-methoxybenzene $\mathbf{2 g}$}

$\mathrm{MeO} \mathrm{N}_{3}$ The product was prepared using procedure E with $1 \mathrm{mmol}$ 3-Methoxybenzyl bromide $(143 \mu \mathrm{L})$ and $1.5 \mathrm{mmol}$ sodium azide $(97.5 \mathrm{mg})$ in DMF.

Yield (clear oil): 93\% (151 mg).

Spectroscopic data are in agreement with the literature. ${ }^{11}$

\footnotetext{
${ }^{10}$ Alonso, F.; Moglie, Y.; Radivoy, G.; Yus, M. Eur. J. Org. Chem. 2010, 2010 (10), 1875-1884

${ }^{11}$ Montanari, S.; Scalvini, L.; Bartolini, M.; Belluti, F.; Gobbi, S.; Andrisano, V.; Ligresti, A.; Di Marzo, V.; Rivara, S.; Mor, M.; et al. J. Med. Chem. 2016, 59, 6387-6406.
} 


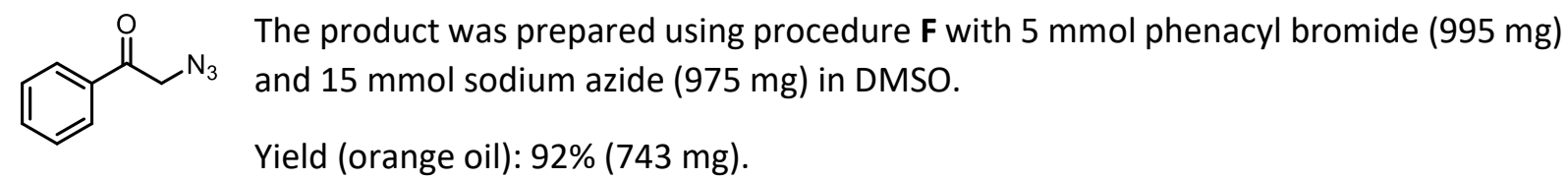

Spectroscopic data are in agreement with the literature. ${ }^{9}$

\section{2-azido-1-(naphthalen-2-yl)ethan-1-one 7b}<smiles>NCC(=O)c1ccc2ccccc2c1</smiles>

The product was prepared using procedure $\mathbf{F}$ with $1 \mathrm{mmol}$ 2-Bromo-2'acetonaphthone (249 $\mathrm{mg}$ ) and $3 \mathrm{mmol}$ sodium azide (195 $\mathrm{mg}$ ) in DMSO.

Yield (orange solid): $94 \%$ (198 mg).

Spectroscopic data are in agreement with the literature. ${ }^{12}$

\section{2-azido-1-(4-methoxyphenyl)ethan-1-one 7c}<smiles>COc1ccc(C(=O)CN)cc1</smiles>

The product was prepared using procedure $\mathbf{F}$ with $1 \mathrm{mmol}$ 2-Bromo-4'methoxyacetophenone $(229 \mathrm{mg})$ and $3 \mathrm{mmol}$ sodium azide $(195 \mathrm{mg})$ in DMSO.

Yield (yellow solid): $95 \%$ (181 mg).

Spectroscopic data are in agreement with the literature. ${ }^{13}$

\section{2-azido-1-(4-nitrophenyl)ethan-1-one 7d}

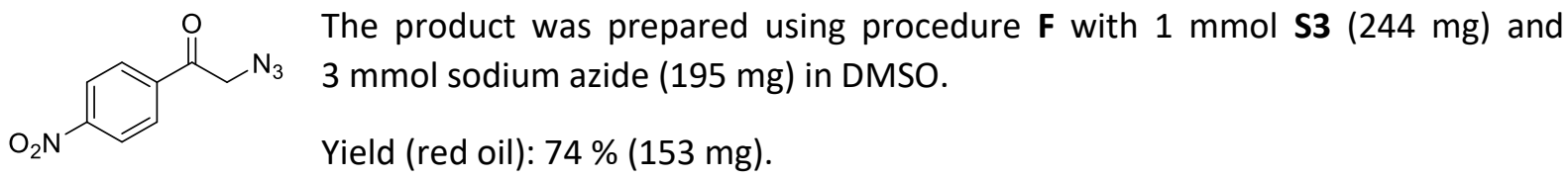

Spectroscopic data are in agreement with the literature. ${ }^{14}$

\footnotetext{
${ }^{12}$ Neyyappadath, R. M.; Chisholm, R.; Greenhalgh, M. D.; Rodríguez-Escrich, C.; Pericàs, M. A.; Hähner, G.; Smith, A. D. ACS Catal. 2018, 8, 1067-1075.

${ }^{13}$ Yokoi, T.; Tanimoto, H.; Ueda, T.; Morimoto, T.; Kakiuchi, K. J. Org. Chem. 2018, 83, 12103-12121.

${ }^{14}$ Gong, P. K.; Blough, B. E.; Brieaddy, L. E.; Huang, X.; Kuhar, M. J.; Navarro, H. A.; Carroll, F. I. J. Med. Chem. 2007, 50 (15), 3686-3695.
} 


\section{1-azido-3,3-dimethylbutan-2-one 7e}

The product was prepared using procedure $\mathbf{G}$ with $1 \mathrm{mmol}$ 1-Chloropinacoline $(131 \mu \mathrm{L})$ and $1.5 \mathrm{mmol}$ sodium azide $(97.5 \mathrm{mg})$ in acetone.

Yield (yellow oil): $79 \%$ (153 mg)

Spectroscopic data are in agreement with the literature. ${ }^{15}$

\section{3-azidobutan-2-one $7 f$}

The product was prepared using procedure $\mathbf{G}$ with $2 \mathrm{mmol}$ 3-Chloro-2-butanone $(202 \mu \mathrm{L})$ $\mathrm{N}_{3}$ and $3 \mathrm{mmol}$ sodium azide $(195 \mathrm{mg})$ in acetone.

Yield (yellow oil): $90 \%$ (203 mg).

Spectroscopic data are in agreement with the literature. ${ }^{16}$

\section{2-azidocyclohexan-1-one $\mathbf{7 g}$}

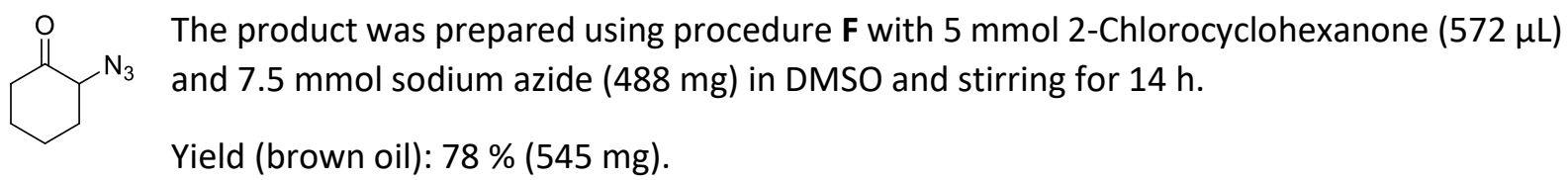

Spectroscopic data are in agreement with the literature. ${ }^{17}$

\footnotetext{
${ }^{15}$ Streefkerk, D. E.; Schmidt, M.; Ippel, J. H.; Hackeng, T. M.; Nuijens, T.; Timmerman, P.; van Maarseveen, J. H. Org. Lett. 2019, 21, 2095-2100.

${ }^{16}$ Peach, P.; Cross, D. J.; Kenny, J. A.; Mann, I.; Houson, I.; Campbell, L.; Walsgrove, T.; Wills, M. Tetrahedron 2006, 62 (8), 1864-1876.

${ }^{17}$ Myers, E. L.; Raines, R. T. A. Angew. Chem. Int. Ed. 2009, 48 (13), 2359-2363.
} 


\section{3-azido-1-phenylpropan-1-one $7 \mathrm{~h}$}

Nors

The product was prepared using procedure $\mathbf{F}$ with $5 \mathrm{mmol}$ 3-Chloropropiophenone $(843 \mathrm{mg}$ ) and $7.5 \mathrm{mmol}$ sodium azide $(488 \mathrm{mg}$ ) in DMSO and stirring for $14 \mathrm{~h}$. Additional purification via column chromatography on silica gel (heptane/EtOAc 9:1)

was performed.

Yield (yellow oil): 68 \% (598 mg).

Spectroscopic data are in agreement with the literature. ${ }^{18}$

\section{$\underline{\text { tert-butyl 2-azidoacetate } \mathbf{7 i}}$}

K I The product was prepared using procedure $\mathbf{F}$ with $5 \mathrm{mmol}$ tert-butyl bromoacetate $(729 \mu \mathrm{L})$ and $7.5 \mathrm{mmol}$ sodium azide $(488 \mathrm{mg})$ in DMSO and stirring for $14 \mathrm{~h}$.

Yield (yellow oil): $56 \%$ (442 mg).

Spectroscopic data are in agreement with the literature. ${ }^{19}$

\footnotetext{
${ }^{18}$ Singh, P. N. D.; Muthukrishnan, S.; Murthy, R. S.; Klima, R. F.; Mandel, S. M.; Hawk, M.; Yarbrough, N.; Gudmundsdóttir, A. D. Tetrahedron Lett. 2003, 44, 9169-9171.

${ }^{19}$ Asano, K.; Matsubara, S. Org. Lett. 2010, 12, 4988-4991.
} 


\section{Amidines, Oxazines and Oxazinones}

General Procedure H

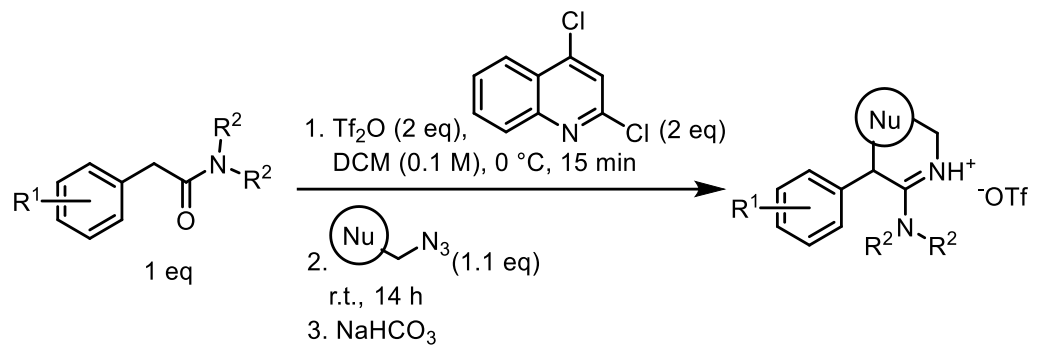

To a mixture of amide (1.0 eq.), 2,4-dichloroquinoline (2.0 eq.) in anhydrous DCM (0.1 M), triflic anhydride (2.0 eq.) was added dropwise under vigorous stirring at $0{ }^{\circ} \mathrm{C}$. After $15 \mathrm{~min}$, the azide (1.1 eq.) was added and the mixture was stirred at r.t. for $14 \mathrm{~h}$. The reaction was then quenched with a saturated aqueous solution of sodium bicarbonate for $1 \mathrm{~h}$. After separation of the biphasic system and extraction of the aqueous phase with DCM $(3 x)$, the organic phases were combined and dried over anhydrous sodium sulfate. The dried solution was filtered and concentrated under reduced pressure. 
5-phenyl-4-(pyrrolidin-1-yl)-2,5-dihydro-1H-benzo[d]azepin-3-ium-trifluoromethanesulfonate 3a<smiles></smiles>

The amidine was prepared using procedure $\mathbf{H}$ with $0.20 \mathrm{mmol}$ amide $1 \mathrm{a}(37.9 \mathrm{mg})$, $0.40 \mathrm{mmol} \mathrm{Tf} \mathrm{f}_{2} \mathrm{O}(67 \mu \mathrm{L}), 0.40 \mathrm{mmol}$ 2,4-dichloroquinoline $(79.2 \mathrm{mg})$ and $0.22 \mathrm{mmol}$ azide 2 a $(32.4 \mathrm{mg})$. Purification was performed with column chromatography on silica gel (DMA/DCM gradient, 5:95 to 30:70).

Yield (orange solid): $78 \%$ (69 mg).

\section{Gram Scale Reaction}

To a mixture of amide $1 \mathrm{a}$ (757 mg, $4.0 \mathrm{mmol}, 1.0$ eq.), 2,4-dichloroquinoline (1.58 g, $8.0 \mathrm{mmol}, 2.0 \mathrm{eq}$.) in anhydrous DCM $(40 \mathrm{~mL})$, triflic anhydride $(1.35 \mathrm{~mL}, 8.0 \mathrm{mmol}, 2.0$ eq.) was added dropwise under vigorous stirring at $0{ }^{\circ} \mathrm{C}$. After $15 \mathrm{~min}$, azide $2 \mathrm{a}(648 \mathrm{mg}, 4.4 \mathrm{mmol}, 1.1 \mathrm{eq}$.) was added and the mixture was stirred at r.t. for $14 \mathrm{~h}$. The reaction was then quenched with a saturated aqueous solution of sodium bicarbonate for $1 \mathrm{~h}$. After separation of the biphasic system and extraction of the aqueous phase with DCM $(3 \times)$, the organic phases were combined and dried over anhydrous sodium sulfate. The dried solution was filtered and concentrated under reduced pressure. Purification was performed with column chromatography on silica gel (DMA/DCM gradient, 5:95 to 30:70). The product was obtained as an orange solid (1.45 g, 83\%).

${ }^{1} \mathrm{H}$ NMR $\left(600 \mathrm{MHz}, \mathrm{CDCl}_{3}\right): \delta(\mathrm{ppm}) 8.94$ (br.s, $\left.1 \mathrm{H}\right), 7.38(\mathrm{~m}, 1 \mathrm{H}), 7.34(\mathrm{~m}, 2 \mathrm{H}), 7.31(\mathrm{t}, J=7.3 \mathrm{~Hz}, 2 \mathrm{H}), 7.27-7.23$ $(\mathrm{m}, 1 \mathrm{H}), 7.21(\mathrm{~d}, J=7.6 \mathrm{~Hz}, 1 \mathrm{H}), 6.88(\mathrm{~d}, J=8.0 \mathrm{~Hz}, 2 \mathrm{H}), 5.44(\mathrm{~s}, 1 \mathrm{H}), 4.01-3.86(\mathrm{~m}, 2 \mathrm{H}), 3.80(\mathrm{dt}, J=12.6,6.5 \mathrm{~Hz}$, $1 \mathrm{H}), 3.75-3.65(\mathrm{~m}, 1 \mathrm{H}), 3.53-3.41(\mathrm{~m}, 2 \mathrm{H}), 3.17(\mathrm{~m}, 1 \mathrm{H}), 2.96(\mathrm{dt}, J=17.8,3.5 \mathrm{~Hz}, 1 \mathrm{H}), 2.30-2.03(\mathrm{~m}, 4 \mathrm{H})$.

${ }^{13} \mathrm{C}$ NMR $\left(151 \mathrm{MHz}, \mathrm{CDCl}_{3}\right): \delta$ (ppm) 164.8, 137.7, 137.0, 133.0, 132.4, 129.8 (2C), 129.7, 129.6, 128.4, 127.6, $125.8(2 \mathrm{C}), 52.6,51.1,49.6,40.5,32.1,25.5,25.1$.

${ }^{19} \mathrm{~F}$ NMR $\left(565 \mathrm{MHz}, \mathrm{CDCl}_{3}\right): \delta(\mathrm{ppm})-78.2$.

HRMS (ESI) $\mathrm{m} / \mathrm{z}$ calculated for $[\mathrm{M}-\mathrm{TfO}]{ }^{+} \mathrm{C}_{20} \mathrm{H}_{23} \mathrm{~N}_{2}{ }^{+}: 291.1856$, found 291.1858 .

ATR-FTIR $\left(\mathrm{cm}^{-1}\right)$ : 3295, 3233, 3066, 2962, 2929, 1642, 1496, 1451, 1279, 1157, 1030, 672.

Mp.: $178-180^{\circ} \mathrm{C}$

\section{4-(diethylamino)-5-phenyl-2,5-dihydro-1H-benzo[d]azepin-3-ium-trifluoromethanesulfonate $3 \mathbf{b}$}<smiles>CCN(CC)C1C(c2ccccc2)c2ccccc2CC[NH+]1Br</smiles>

The amidine was prepared using procedure $\mathbf{H}$ with $0.20 \mathrm{mmol}$ amide $\mathbf{1 b}$ (38.3 $\mathrm{mg}$ ), $0.40 \mathrm{mmol} \mathrm{Tf}_{2} \mathrm{O}(67 \mu \mathrm{L}), 0.40 \mathrm{mmol}$ 2,4-dichloroquinoline $(79.2 \mathrm{mg}$ ) and $0.22 \mathrm{mmol}$ azide 2 a $(32.4 \mathrm{mg})$. Purification was performed with column chromatography on silica gel (DMA/DCM gradient, 5:95 to 30:70).

Yield (orange-brown oil): 51 \% (45 mg)

${ }^{1} \mathrm{H}$ NMR $\left(600 \mathrm{MHz}, \mathrm{CDCl}_{3}\right): \delta(\mathrm{ppm}) 9.13$ (br.s, $\left.1 \mathrm{H}\right), 7.43-7.38(\mathrm{~m}, 1 \mathrm{H}), 7.38-7.29(\mathrm{~m}, 4 \mathrm{H}), 7.28(\mathrm{~m}, 1 \mathrm{H}), 7.18(\mathrm{~d}$, $J=7.5 \mathrm{~Hz}, 1 \mathrm{H}), 6.84(\mathrm{~d}, J=7.7 \mathrm{~Hz}, 2 \mathrm{H}), 5.42(\mathrm{~s}, 1 \mathrm{H}), 3.95-3.76(\mathrm{~m}, 2 \mathrm{H}), 3.68(\mathrm{~m}, 1 \mathrm{H}), 3.61(\mathrm{~m}, 2 \mathrm{H}), 3.49-3.34$ $(\mathrm{m}, 1 \mathrm{H}), 3.32-3.16(\mathrm{~m}, 1 \mathrm{H}), 2.99-2.91(\mathrm{~m}, 1 \mathrm{H}), 1.43(\mathrm{t}, J=7.1 \mathrm{~Hz}, 3 \mathrm{H}), 1.38(\mathrm{t}, J=7.1 \mathrm{~Hz}, 3 \mathrm{H})$.

${ }^{13} \mathrm{C}$ NMR (151 MHz, CDCl $): \delta$ (ppm) 166.4, 137.7 (2C), 133.2, 132.8, 129.9 (2C), 129.7, 129.3, 128.4, 127.7, 125.5 (2C), 50.3, 47.7, 46.2, 40.0, 32.1, 14.9, 11.7.

${ }^{19} \mathrm{~F}$ NMR $\left(565 \mathrm{MHz}, \mathrm{CDCl}_{3}\right): \delta$ (ppm) -78.2. 
HRMS (ESI) m/z calculated for [M-TfO] ${ }^{+} \mathrm{C}_{20} \mathrm{H}_{25} \mathrm{~N}_{2}{ }^{+}:$293.2012, found 293.2013.

ATR-FTIR (cm-1): 3280, 3229, 2982, 2928, 16,31, 1584, 1495, 1449, 1243, 1224, 1198, 1154, 1029, 748, 636.

\section{4-(azetidin-1-yl)-5-phenyl-2,5-dihydro-1H-benzo[d]azepin-3-ium-trifluoromethanesulfonate $3 \mathrm{c}$}<smiles>[O-][NH+]1CCc2ccccc2C(c2ccccc2)C1N1CCC1</smiles>

The amidine was prepared using procedure $\mathbf{H}$ with $0.20 \mathrm{mmol}$ amide $1 \mathrm{c}(35.0 \mathrm{mg})$, $0.40 \mathrm{mmol} \mathrm{Tf}{ }_{2} \mathrm{O}(67 \mu \mathrm{L}), 0.40 \mathrm{mmol}$ 2,4-dichloroquinoline (79.2 $\mathrm{mg}$ ) and $0.22 \mathrm{mmol}$ azide $2 \mathrm{a}$ (32.4 mg). Purification was performed with column chromatography on silica gel (DMA/DCM gradient, 5:95 to 30:70).

Yield (orange solid): $69 \%$ (59 mg).

${ }^{1} \mathrm{H}$ NMR $\left(600 \mathrm{MHz}, \mathrm{CDCl}_{3}\right): \delta(\mathrm{ppm}) 8.90(\mathrm{~s}, 1 \mathrm{H}), 7.34-7.27(\mathrm{~m}, 3 \mathrm{H}), 7.25(\mathrm{~m}, 2 \mathrm{H}), 7.16(\mathrm{~d}, J=7.6 \mathrm{~Hz}, 1 \mathrm{H}), 7.12(\mathrm{~d}$, $J=7.5 \mathrm{~Hz}, 1 \mathrm{H}), 6.87(\mathrm{~d}, J=7.9 \mathrm{~Hz}, 2 \mathrm{H}), 4.86(\mathrm{~s}, 1 \mathrm{H}), 4.52(\mathrm{~m}, 3 \mathrm{H}), 4.43(\mathrm{~m}, 1 \mathrm{H}), 3.37(\mathrm{~m}, 2 \mathrm{H}), 3.00(\mathrm{ddd}, J=15.5$, $10.5,4.6 \mathrm{~Hz}, 1 \mathrm{H}), 2.92-2.85(\mathrm{~m}, 1 \mathrm{H}), 2.53(\mathrm{~m}, 1 \mathrm{H}), 2.48-2.37(\mathrm{~m}, 1 \mathrm{H})$.

${ }^{13} \mathrm{C}$ NMR (151 MHz, CDCl 3$): \delta$ (ppm) 164.6, 137.6, 136.5, 132.5, 132.1, 130.1, 129.7 (2C), 129.6, 128.4, 127.7, $126.2(2 \mathrm{C}), 53.2,52.3,49.3,40.8,32.0,14.8$.

${ }^{19}$ F NMR $\left(565 \mathrm{MHz}, \mathrm{CDCl}_{3}\right): \delta(\mathrm{ppm})-78.3$.

HRMS (ESI) m/z calculated for [M-TfO] ${ }^{+} \mathrm{C}_{19} \mathrm{H}_{21} \mathrm{~N}_{2}{ }^{+}:$277.1699, found 277.1697.

ATR-FTIR (cm $\left.{ }^{-1}\right)$ : 3219, 3092, 2950, 1656, 1495, 1448, 1244, 1157, 1029, 725, 636, 517.

Mp.: $163-165^{\circ} \mathrm{C}$.

5-(2-bromophenyl)-4-(pyrrolidin-1-yl)-2,5-dihydro-1H-benzo[d]azepin-3-ium-trifluoromethanesulfonate $3 d$<smiles>Brc1ccccc1C1C(N2CCCC2)=[NH+]CCc2ccccc21</smiles>

The amidine was prepared using procedure $\mathbf{H}$ with $0.20 \mathrm{mmol}$ amide $\mathbf{1 d}(53.6 \mathrm{mg})$, $0.40 \mathrm{mmol} \mathrm{Tf} \mathrm{O}_{2} \mathrm{O}(67 \mu \mathrm{L}), 0.40 \mathrm{mmol}$ 2,4-dichloroquinoline $(79.2 \mathrm{mg})$ and $0.22 \mathrm{mmol}$ azide 2 a (32.4 mg). Purification was performed with column chromatography on silica gel (DMA/DCM gradient, 5:95 to 30:70).

Yield (yellow solid): $70 \%$ (73 mg).

${ }^{1} \mathrm{H}$ NMR $\left(600 \mathrm{MHz}, \mathrm{CDCl}_{3}\right) \delta(\mathrm{ppm}) 8.81$ (br.s, $\left.1 \mathrm{H}\right), 7.67$ (d, J= 7.5 Hz, 1H), $7.46(\mathrm{t}, J=7.6 \mathrm{~Hz}, 1 \mathrm{H}), 7.34(\mathrm{~m}, 2 \mathrm{H})$, $7.28-7.22(\mathrm{~m}, 2 \mathrm{H}), 7.19(\mathrm{~d}, J=7.4 \mathrm{~Hz}, 1 \mathrm{H}), 6.76-6.61(\mathrm{~m}, 1 \mathrm{H}), 5.21(\mathrm{~s}, 1 \mathrm{H}), 4.29(\mathrm{~m}, 1 \mathrm{H}), 3.75(\mathrm{~m}, 2 \mathrm{H}), 3.63-$ $3.52(\mathrm{~m}, 2 \mathrm{H}), 3.44-3.30(\mathrm{~m}, 2 \mathrm{H}), 3.01(\mathrm{~m}, 1 \mathrm{H}), 2.23-2.01(\mathrm{~m}, 4 \mathrm{H})$.

${ }^{13} \mathrm{C}$ NMR $\left(151 \mathrm{MHz}, \mathrm{CDCl}_{3}\right): \delta$ (ppm) 163.8, 138.0, 137.1, 134.6, 133.0, 132.0, 131.1, 130.4, 130.1, 128.3, 128.1, $128.1,121.6,53.2,50.7,49.7,38.9,32.3,25.4,24.9$.

${ }^{19} \mathrm{~F}$ NMR $\left(565 \mathrm{MHz}, \mathrm{CDCl}_{3}\right): \delta(\mathrm{ppm})-78.2$.

HRMS (ESI) m/z calculated for [M-TfO] ${ }^{+} \mathrm{C}_{20} \mathrm{H}_{22} \mathrm{BrN}_{2}{ }^{+}: 371.0940$, found 371.0954 .

ATR-FTIR (cm $\left.{ }^{-1}\right)$ : 3287, 3220, 3071, 2980, 2960, 2929, 1637, 1466, 1244, 1157, 1029, 757, 729, 636.

Mp.: $212-215^{\circ} \mathrm{C}$. 
5-(4-methoxyphenyl)-4-(pyrrolidin-1-yl)-2,5-dihydro-1H-benzo[d]azepin-3-ium- trifluoromethanesulfonate $3 e$<smiles>COc1ccc(C2(N3CCCC3)Cc3ccccc3CC2O)cc1</smiles>
The amidine was prepared using procedure $\mathbf{H}$ with $0.20 \mathrm{mmol}$ amide $\mathbf{1 e}$ (43.9 $\mathrm{mg}$ ), $0.40 \mathrm{mmol} \mathrm{Tf} 2 \mathrm{O}(67 \mu \mathrm{L}$ ), $0.40 \mathrm{mmol}$ 2,4-dichloroquinoline (79.2 mg) and $0.22 \mathrm{mmol}$ azide $2 \mathrm{a}(32.4 \mathrm{mg})$. Purification was performed with column chromatography on silica gel (DMA/DCM gradient, 5:95 to 30:70).

Yield (orange-brown oil): $45 \%$ (42 mg)

${ }^{1} \mathrm{H}$ NMR $\left(400 \mathrm{MHz}, \mathrm{CDCl}_{3}\right): \delta(\mathrm{ppm}) 9.03$ (br.s, $\left.1 \mathrm{H}\right), 7.39(\mathrm{~m}, 1 \mathrm{H}), 7.33-7.22(\mathrm{~m}, 3 \mathrm{H}), 7.16(\mathrm{~d}, J=7.6 \mathrm{~Hz}, 1 \mathrm{H}), 6.87$ $(\mathrm{d}, J=8.8 \mathrm{~Hz}, 2 \mathrm{H}), 6.79(\mathrm{~d}, J=8.2 \mathrm{~Hz}, 2 \mathrm{H}), 5.36(\mathrm{~s}, 1 \mathrm{H}), 3.95-3.85(\mathrm{~m}, 2 \mathrm{H}), 3.85-3.80(\mathrm{~m}, 1 \mathrm{H}), 3.79(\mathrm{~s}, 3 \mathrm{H}), 3.76$ $(\mathrm{m}, 1 \mathrm{H}), 3.54(\mathrm{~m}, 2 \mathrm{H}), 3.24-3.10(\mathrm{~m}, 1 \mathrm{H}), 2.98(\mathrm{~m}, 1 \mathrm{H}), 2.26-2.06(\mathrm{~m}, 4 \mathrm{H})$.

${ }^{13} \mathrm{C}$ NMR $(151 \mathrm{MHz}, \mathrm{CDCl} 3): \delta$ (ppm) 164.9, 159.5, 137.8, 132.8, 132.4, 130.0, 129.6, 128.7, 127.5, 127.0 (2C), $115.1(2 \mathrm{C}), 55.5,52.0,51.0,49.6,40.5,32.1,25.50,25.1$.

${ }^{19} \mathrm{~F}$ NMR $\left(565 \mathrm{MHz}, \mathrm{CDCl}_{3}\right): \delta(\mathrm{ppm})-78.2$.

HRMS (ESI) $\mathrm{m} / \mathrm{z}$ calculated for [M-TfO] ${ }^{+} \mathrm{C}_{21} \mathrm{H}_{25} \mathrm{~N}_{2} \mathrm{O}^{+}: 321.1961$, found 321.1962 .

ATR-FTIR $\left(\mathrm{cm}^{-1}\right)$ : 3293, 3226, 3064, 2959, 2935, 1639, 1510, 1455, 1242, 1153, 1027, 732, 635, 516.

5-(4-nitrophenyl)-4-(pyrrolidin-1-yl)-2,5-dihydro-1H-benzo[d]azepin-3-ium- trifluoromethanesulfonate $\mathbf{3 f}$<smiles>O=[N+]([O-])c1ccc(C2c3ccccc3CC[NH+]2N2CCCC2)cc1</smiles>

The amidine was prepared using procedure $\mathbf{H}$ with $0.20 \mathrm{mmol}$ amide $1 \mathrm{f}$ (46.9 mg), $0.40 \mathrm{mmol} \mathrm{Tf} \mathrm{O}_{2} \mathrm{O}(67 \mu \mathrm{L}$ ), $0.40 \mathrm{mmol}$ 2,4-dichloroquinoline (79.2 mg) and $0.22 \mathrm{mmol}$ azide $2 \mathrm{a}(32.4 \mathrm{mg})$. Purification was performed with column chromatography on silica gel (DMA/DCM gradient, 0:100 to 30:70).

Yield (orange-brown oil): $13 \%$ (13 mg)

${ }^{1} \mathrm{H}$ NMR $\left(600 \mathrm{MHz}, \mathrm{CDCl}_{3}\right): \delta(\mathrm{ppm}) 9.18$ (br.s, $\left.1 \mathrm{H}\right), 8.20(\mathrm{~d}, J=12.5 \mathrm{~Hz}, 2 \mathrm{H}), 7.49-7.41(\mathrm{~m}, 1 \mathrm{H}), 7.36(\mathrm{dd}, J=7.5$, $J=7.5 \mathrm{~Hz}, 1 \mathrm{H}), 7.31(\mathrm{~d}, J=6.6 \mathrm{~Hz}, 1 \mathrm{H}), 7.21(\mathrm{~d}, J=10.1 \mathrm{~Hz}, 1 \mathrm{H}), 7.16(\mathrm{~d}, J=8.5 \mathrm{~Hz}, 2 \mathrm{H}), 5.46(\mathrm{~s}, 1 \mathrm{H}), 3.97(\mathrm{~m}, 1 \mathrm{H})$, $3.95-3.88(\mathrm{~m}, 2 \mathrm{H}), 3.81-3.72(\mathrm{~m}, 1 \mathrm{H}), 3.57(\mathrm{~m}, 1 \mathrm{H}), 3.34(\mathrm{~m}, 1 \mathrm{H}), 3.29-3.17(\mathrm{~m}, 1 \mathrm{H}), 2.98(\mathrm{~m}, 1 \mathrm{H}), 2.36-2.06$ $(\mathrm{m}, 4 \mathrm{H})$.

${ }^{13} \mathrm{C}$ NMR (151 MHz, CDCl $): \delta$ (ppm) 163.7, 147.8, 144.1, 137.6, 132.9 (2C), 130.4, 128.4, 128.0, 127.2 (2C), $124.9(2 \mathrm{C}), 52.5,51.3,50.0,40.7,32.1,25.5,25.1$.

${ }^{19} \mathrm{~F}$ NMR $\left(565 \mathrm{MHz}, \mathrm{CDCl}_{3}\right): \delta(\mathrm{ppm})-78.2$.

HRMS (ESI) $\mathrm{m} / \mathrm{z}$ calculated for $[\mathrm{M}-\mathrm{TfO}]^{+} \mathrm{C}_{20} \mathrm{H}_{22} \mathrm{~N}_{3} \mathrm{O}_{2}{ }^{+}: 336.1707$, found 336.1711 .

ATR-FTIR $\left(\mathrm{cm}^{-1}\right)$ : 3230, 3080, 2960, 2931, 16,45, 1521, 1347, 1159, 1030, 637. 


\section{2-(4-nitrophenyl)-2-(phenethylamino)-1-(pyrrolidin-1-yl)ethan-1-one S4}<smiles>O=C(NCCc1ccccc1)C(c1ccccc1)(c1ccc([N+](=O)[O-])cc1)N1CCCC1</smiles>

The $\alpha$-aminated product was formed as the major product during the synthesis of $\mathbf{3 f}$. Purification was performed with column chromatography on silica gel (DMA/DCM gradient, 0:100 to 30:70).

Yield (orange oil): $37 \%$ (26 mg).

${ }^{1} \mathrm{H}$ NMR $\left(600 \mathrm{MHz}, \mathrm{CDCl}_{3}\right): \delta(\mathrm{ppm}) 8.16(\mathrm{~d}, J=8.7 \mathrm{~Hz}, 2 \mathrm{H}), 7.47(\mathrm{~d}, J=8.7 \mathrm{~Hz}, 2 \mathrm{H}), 7.27(\mathrm{~m}, 2 \mathrm{H}), 7.23-7.12(\mathrm{~m}$, $3 \mathrm{H}), 4.43(\mathrm{~s}, 1 \mathrm{H}), 3.59-3.51(\mathrm{~m}, 1 \mathrm{H}), 3.47(\mathrm{~m}, 1 \mathrm{H}), 3.43-3.35(\mathrm{~m}, 1 \mathrm{H}), 3.09(\mathrm{~m}, 1 \mathrm{H}), 2.88-2.74(\mathrm{~m}, 3 \mathrm{H}), 2.65$ (m, 1H), $2.37-2.17$ (br.s, $1 \mathrm{H}), 1.98-1.71(\mathrm{~m}, 4 \mathrm{H})$.

${ }^{13} \mathrm{C}$ NMR $\left(151 \mathrm{MHz}, \mathrm{CDCl}_{3}\right): \delta$ (ppm) 169.4, 147.7, 146.1, 139.8, $128.9(2 \mathrm{C}), 128.8(2 \mathrm{C}), 128.6(2 \mathrm{C}), 126.4$, 124.1 (2C), 64.1, 49.3, 46.3, 46.2, 36.9, 26.1, 24.1.

HRMS (ESI) m/z calculated for $[\mathrm{M}-\mathrm{H}]^{+} \mathrm{C}_{20} \mathrm{H}_{24} \mathrm{~N}_{3} \mathrm{O}_{3}{ }^{+}: 354,1812$, found 354.1813 .

$\operatorname{ATR}-\mathrm{FTIR}\left(\mathrm{cm}^{-1}\right)$ : 3330, 3026, 2949, 2877, 1640, 1604, 1519, 1429, 1345, 1109, 842, 752, 731, 700.

4-(pyrrolidin-1-yl)-5-(4-(trifluoromethyl)phenyl)-2,5-dihydro-1H-benzo[d]azepin-3-ium- trifluoromethanesulfonate $3 g$<smiles></smiles>

The amidine was prepared using procedure $\mathbf{H}$ with $0.20 \mathrm{mmol}$ amide $\mathbf{1 g}$ (51.5 mg), $0.40 \mathrm{mmol} \mathrm{Tf} \mathrm{O}_{2} \mathrm{O}(67 \mu \mathrm{L}$ ), $0.40 \mathrm{mmol}$ 2,4-dichloroquinoline (79.2 mg) and $0.22 \mathrm{mmol}$ azide $2 \mathrm{a}(32.4 \mathrm{mg})$. Purification was performed with column chromatography on silica gel (DMA/DCM gradient, 5:95 to 30:70).

Yield (orange-brown oil): $45 \%$ (46 mg).

${ }^{1} \mathrm{H}$ NMR $\left(600 \mathrm{MHz}, \mathrm{CDCl}_{3}\right): \delta(\mathrm{ppm}) 9.01$ (br.s, $\left.1 \mathrm{H}\right), 7.56(\mathrm{~d}, J=8.3 \mathrm{~Hz}, 2 \mathrm{H}), 7.36(\mathrm{~m}, 1 \mathrm{H}), 7.27(\mathrm{~m}, 1 \mathrm{H}), 7.21(\mathrm{~d}, J=$ $6.4 \mathrm{~Hz}, 1 \mathrm{H}), 7.14(\mathrm{~d}, J=7.2 \mathrm{~Hz}, 1 \mathrm{H}), 7.02(\mathrm{~d}, J=8.2 \mathrm{~Hz}, 2 \mathrm{H}), 5.38(\mathrm{~s}, 1 \mathrm{H}), 3.96-3.73(\mathrm{~m}, 3 \mathrm{H}), 3.73-3.61(\mathrm{~m}, 1 \mathrm{H})$, $3.51-3.39(m, 1 H), 3.32(m, 1 H), 3.18-3.04(m, 1 H), 2.90(m, 1 H), 2.28-1.97(m, 4 H)$.

${ }^{13} \mathrm{C}$ NMR $\left(151 \mathrm{MHz}, \mathrm{CDCl}_{3}\right): \delta$ (ppm) 164.0, 141.1, 137.6, 132.9, 132.6, 130.8 (q, $J=33.2 \mathrm{~Hz}, 1 \mathrm{C}$ ), 130.1, 128.9, $126.7(q, J=3.0 \mathrm{~Hz}, 2 \mathrm{C}), 126.5(2 \mathrm{C}), 122.2(\mathrm{dd}, J=320.1 \mathrm{~Hz}, J=487.7 \mathrm{~Hz}, 1 \mathrm{C}), 52.5,51.2,49.9,40.7,32.0,25.4$, 25.1.

${ }^{19} \mathrm{~F}$ NMR $\left(565 \mathrm{MHz}, \mathrm{CDCl}_{3}\right): \delta(\mathrm{ppm})-62.76,-78.3$.

HRMS (ESI) $\mathrm{m} / \mathrm{z}$ calculated for $[\mathrm{M}-\mathrm{TfO}]^{+} \mathrm{C}_{21} \mathrm{H}_{22} \mathrm{~F}_{3} \mathrm{~N}_{2}{ }^{+}: 359,1730$, found 359.1729 .

$\operatorname{ATR-FTIR}\left(\mathrm{cm}^{-1}\right)$ : 3295, 3229, 3080, 2961, 2930, 1644, 1327, 1246, 1161, 1121, 1069, 1030, 638. 
9-methyl-5-phenyl-4-(pyrrolidin-1-yl)-2,5-dihydro-1H-benzo[d]azepin-3-ium- trifluoromethanesulfonate $3 h$

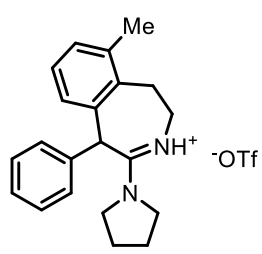

The amidine was prepared using procedure $\mathbf{H}$ with $0.20 \mathrm{mmol}$ amide $1 \mathbf{a}(37.9 \mathrm{mg})$, $0.40 \mathrm{mmol} \mathrm{Tf}_{2} \mathrm{O}(67 \mu \mathrm{L}), 0.40 \mathrm{mmol}$ 2,4-dichloroquinoline $(79.2 \mathrm{mg})$ and $0.22 \mathrm{mmol}$ azide $\mathbf{2 b}(35.5 \mathrm{mg})$. Purification was performed with column chromatography on silica gel (DMA/DCM gradient, 5:95 to 30:70).

Yield (orange-brown solid): $61 \%$ (55 mg).

${ }^{1} \mathbf{H}$ NMR $\left(600 \mathrm{MHz}, \mathrm{CDCl}_{3}\right): \delta(\mathrm{ppm}) 9.14$ (br.s, $\left.1 \mathrm{H}\right), 7.38-7.27(\mathrm{~m}, 4 \mathrm{H}), 7.22(\mathrm{dd}, J=17.4,9.8 \mathrm{~Hz}, 1 \mathrm{H}), 7.03(\mathrm{~d}$, $J=7.5 \mathrm{~Hz}, 1 \mathrm{H}), 6.89(\mathrm{~d}, J=8.1 \mathrm{~Hz}, 2 \mathrm{H}), 5.43(1 \mathrm{H}), 3.95(\mathrm{~m}, 1 \mathrm{H}), 3.89-3.79(\mathrm{~m}, 2 \mathrm{H}), 3.79-3.68(\mathrm{~m}, 1 \mathrm{H}), 3.59(\mathrm{~m}$, $1 \mathrm{H}), 3.52-3.43(\mathrm{~m}, 1 \mathrm{H}), 3.02(\mathrm{ddd}, J=17.9,12.8,5.0 \mathrm{~Hz}, 1 \mathrm{H}), 2.74(\mathrm{~m}, 1 \mathrm{H}), 2.32-2.23(\mathrm{~m}, 3 \mathrm{H}), 2.24-2.07(\mathrm{~m}$, $4 \mathrm{H})$.

${ }^{13} \mathrm{C}$ NMR (151 MHz, CDCl 3 ): $\delta$ (ppm) 165.0, 140.1, 137.3, 136.1, 131.7, 131.2, 129.8 (2C), 129.0, 128.4, 127.3, $125.7(2 \mathrm{C}), 53.0,50.9,49.6,39.9,30.0,25.5,25.2,20.7$.

${ }^{19} \mathrm{~F}$ NMR $\left(565 \mathrm{MHz}, \mathrm{CDCl}_{3}\right): \delta(\mathrm{ppm})-78.2$.

HRMS (ESI) m/z calculated for [M-TfO] ${ }^{+} \mathrm{C}_{21} \mathrm{H}_{25} \mathrm{~N}_{2}{ }^{+}: 305.2012$, found 305.2017.

ATR-FTIR (cm-1): 3220, 3060, 1632, 1443, 1243, 1158, 1029, 731, 700, 636, 517.

Mp.: $243-245^{\circ} \mathrm{C}$.

8-methoxy-5-phenyl-4-(pyrrolidin-1-yl)-2,5-dihydro-1H-benzo[d]azepin-3-ium-trifluoromethanesulfonate $\mathbf{3 i}$

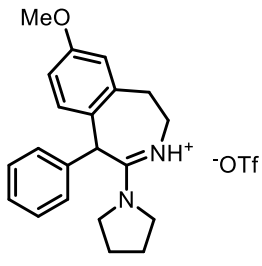

The amidine was prepared using procedure $\mathbf{H}$ with $0.20 \mathrm{mmol}$ amide $1 \mathrm{a}(37.9 \mathrm{mg})$, $0.40 \mathrm{mmol} \mathrm{Tf} \mathrm{O}_{2} \mathrm{O}(67 \mu \mathrm{L}), 0.40 \mathrm{mmol}$ 2,4-dichloroquinoline $(79.2 \mathrm{mg})$ and $0.22 \mathrm{mmol}$ azide $2 \mathrm{c}(39.0 \mathrm{mg})$. Purification was performed with column chromatography on silica gel (DMA/DCM gradient, 5:95 to 30:70).

Yield (orange-brown solid): $93 \%$ (88 mg).

${ }^{1} \mathrm{H}$ NMR $\left(600 \mathrm{MHz}, \mathrm{CDCl}_{3}\right): \delta(\mathrm{ppm}) 8.97$ (br.s, $\left.1 \mathrm{H}\right), 7.39-7.28(\mathrm{~m}, 3 \mathrm{H}), 7.10(\mathrm{~d}, J=8.5,1 \mathrm{H}), 6.88(\mathrm{~d}, J=9.4 \mathrm{~Hz}$, $2 \mathrm{H}), 6.84(\mathrm{dd}, J=8,5,2.8 \mathrm{~Hz}, 1 \mathrm{H}), 6.76(\mathrm{~d}, J=2.6 \mathrm{~Hz}, 1 \mathrm{H}), 5.38(\mathrm{~s}, 1 \mathrm{H}), 3.96-3.86(\mathrm{~m}, 2 \mathrm{H}), 3.82(\mathrm{~s}, 3 \mathrm{H}), 3.80(\mathrm{~m}$, $1 \mathrm{H}), 3.72(\mathrm{~m}, 1 \mathrm{H}), 3.55-3.35(\mathrm{~m}, 2 \mathrm{H}), 3.24-3.05(\mathrm{~m}, 1 \mathrm{H}), 2.94(\mathrm{~m}, 1 \mathrm{H}), 2.28-2.03(\mathrm{~m}, 4 \mathrm{H})$.

${ }^{13} \mathrm{C}$ NMR (151 MHz, CDCl $): \delta$ (ppm) 165.0, 160.3, 139.1, 137.4, 134.2, 129.7 (2C), 128.3, 125.8 (2C), 121.4, 116.9, $113.6,55.5,51.8,51.0,49.6,40.3,32.4,25.5,25.2$

${ }^{19} \mathrm{~F}$ NMR $\left(565 \mathrm{MHz}, \mathrm{CDCl}_{3}\right): \delta(\mathrm{ppm})-78.2$.

HRMS (ESI) m/z calculated for [M-TfO] ${ }^{+} \mathrm{C}_{21} \mathrm{H}_{25} \mathrm{~N}_{2} \mathrm{O}^{+}: 321.1961$, found 321.1961 .

$\operatorname{ATR}$-FTIR $\left(\mathrm{cm}^{-1}\right):$ 3291, 3229, 3062, 2960, 2937, 1639, 1499, 1451, 1242, 1156, 1028, 731, 700, 635, 517.

Mp.: $160-162^{\circ} \mathrm{C}$. 
7-fluoro-5-phenyl-4-(pyrrolidin-1-yl)-2,5-dihydro-1H-benzo[d]azepin-3-ium- trifluoromethanesulfonate $\mathbf{3 j}$<smiles>Fc1ccc2c(c1)C(c1ccccc1)C(N1CCCC1)NCC[O+]2</smiles>

The amidine was prepared using procedure $\mathbf{H}$ with $0.20 \mathrm{mmol}$ amide $1 \mathrm{a}(37.9 \mathrm{mg})$, $0.40 \mathrm{mmol} \mathrm{Tf} \mathrm{O}_{2} \mathrm{O}(67 \mu \mathrm{L}), 0.40 \mathrm{mmol}$ 2,4-dichloroquinoline $(79.2 \mathrm{mg})$ and $0.22 \mathrm{mmol}$ azide $\mathbf{2 d}(36.3 \mathrm{mg})$. Purification was performed with column chromatography on silica gel (DMA/DCM gradient, 5:95 to 30:70).

Yield (orange-brown oil): $39 \%$ (36 mg).

${ }^{1} \mathrm{H}$ NMR $\left(600 \mathrm{MHz}, \mathrm{CDCl}_{3}\right): \delta(\mathrm{ppm}) 9.13$ (br.s, $\left.1 \mathrm{H}\right), 7.43-7.29(\mathrm{~m}, 3 \mathrm{H}), 7.26-7.22(\mathrm{~m}, 1 \mathrm{H}), 7.12(\mathrm{~m}, 1 \mathrm{H}), 6.97-$ $6.92(\mathrm{~m}, 1 \mathrm{H}), 6.88(\mathrm{~m}, 2 \mathrm{H}), 5.37(\mathrm{~s}, 1 \mathrm{H}), 3.97-3.86(\mathrm{~m}, 2 \mathrm{H}), 3.81(\mathrm{~m}, 2 \mathrm{H}), 3.55(\mathrm{~m}, 1 \mathrm{H}), 3.46(\mathrm{~m}, 1 \mathrm{H}), 3.17(\mathrm{~m}, 1 \mathrm{H})$, $2.95(\mathrm{~m}, 1 \mathrm{H}), 2.33-2.02(\mathrm{~m}, 4 \mathrm{H})$.

$\left.{ }^{13} \mathrm{C} \mathrm{NMR} \mathrm{(151} \mathrm{MHz,} \mathrm{CDCl} 3\right): \delta(\mathrm{ppm}) 164.2,161.5$ (d, $\left.J=24.7 \mathrm{~Hz}, 1 \mathrm{C}\right), 136.3,134.2$ (d, $\left.J=7.5 \mathrm{~Hz}, 1 \mathrm{C}\right), 133.5$ (d, $J=$ $4.5 \mathrm{~Hz}, 1 \mathrm{C}$ ), 131.25 (d, J = 6.0 Hz, 1C), 130.0 (2C), 128.7, 125.7 (2C), 119.3 (d, J = 22.6 Hz, 1C), 116.9 (d, J = 21.1 $\mathrm{Hz}, 1 \mathrm{C}), 52.3,51.1,49.8,40.6,31.5,25.5,25.2$.

${ }^{19} \mathrm{~F}$ NMR $\left(565 \mathrm{MHz}, \mathrm{CDCl}_{3}\right): \delta(\mathrm{ppm})-78.3,-115.1$.

HRMS (ESI) $\mathrm{m} / \mathrm{z}$ calculated for [M-TfO] ${ }^{+} \mathrm{C}_{20} \mathrm{H}_{22} \mathrm{FN}_{2}{ }^{+}: 309,1762$, found 359.1773 .

ATR-FTIR $\left(\mathrm{cm}^{-1}\right)$ : 3295, 3229, 3064, 2961, 2928, 1640, 1498, 1450, 1241, 1152, 1060, 733, 699, 635, 516.

4-phenyl-3-(pyrrolidin-1-yl)-1,4-dihydroisoquinolin-2-ium-trifluoro-methanesulfonate 3 I

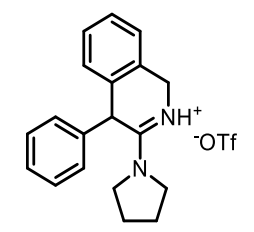

The amidine was prepared using procedure $\mathbf{H}$ with $0.40 \mathrm{mmol}$ amide $1 \mathrm{a}(75.7 \mathrm{mg})$, $0.80 \mathrm{mmol} \mathrm{Tf}_{2} \mathrm{O}(135 \mu \mathrm{L}), 0.80 \mathrm{mmol}$ 2,4-dichloroquinoline (158 $\mathrm{mg}$ ) and $0.44 \mathrm{mmol}$ azide $\mathbf{2 f}(64.8 \mathrm{mg})$. Purification was performed with column chromatography on silica gel (DMA/DCM gradient, 5:95 to 30:70).

Yield (orange-brown oil): $49 \%$ (87 mg).

${ }^{1} \mathrm{H}$ NMR $\left(600 \mathrm{MHz}, \mathrm{CDCl}_{3}\right): \delta$ (ppm) 9.74 (br.s, $\left.1 \mathrm{H}\right), 7.40-7.29(\mathrm{~m}, 6 \mathrm{H}), 7.24(\mathrm{~d}, J=7.3 \mathrm{~Hz}, 1 \mathrm{H}), 7.19(\mathrm{~d}, J=7.5 \mathrm{~Hz}$, $2 \mathrm{H}), 5.15(\mathrm{~s}, 1 \mathrm{H}), 4.86(\mathrm{~d}, J=15.9 \mathrm{~Hz}, 1 \mathrm{H}), 4.65(\mathrm{~d}, J=17.2 \mathrm{~Hz}, 1 \mathrm{H}), 3.87-3.78(\mathrm{~m}, 3 \mathrm{H}), 3.53-3.46(\mathrm{~m}, 1 \mathrm{H})$, $2.18-2.05(\mathrm{~m}, 3 \mathrm{H}), 2.00-1.93(\mathrm{~m}, 1 \mathrm{H})$.

${ }^{13} \mathrm{C}$ NMR (151 MHz, CDCl 13 ): $\delta$ (ppm) 161.7, 135.5, 132.0, 130.2 (2C), 129.8, 128.9, 128.8, 128.5, 127.8, 126.7 (2C), $126.5,49.9,49.5,47.9,45.4,25.5,24.9$.

${ }^{19} \mathrm{~F}$ NMR $\left(565 \mathrm{MHz}, \mathrm{CDCl}_{3}\right): \delta(\mathrm{ppm})-78.2$.

HRMS (ESI) $\mathrm{m} / \mathrm{z}$ calculated for [M-TfO] ${ }^{+} \mathrm{C}_{19} \mathrm{H}_{21} \mathrm{~N}_{2}{ }^{+}: 277.1699$, found 277.1712 .

ATR-FTIR $\left(\mathrm{cm}^{-1}\right):$ 3213. 2859, 1653, 1457, 1223, 1154, 1027, 910, 727, 601. 
methoxy-4-phenyl-3-(pyrrolidin-1-yl)-1,4-dihydroisoquinolin-2-ium trifluoromethanesulfonate (regioisomer mixture) $3 \mathrm{~m}$

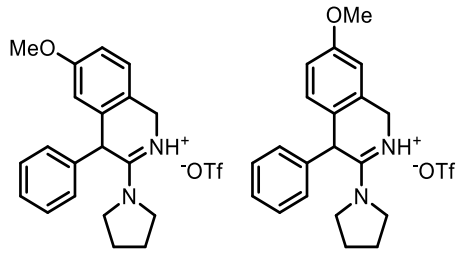

The amidine was prepared using procedure $\mathbf{H}$ with $0.40 \mathrm{mmol}$ amide 1a $(75.7 \mathrm{mg}), \quad 0.80 \mathrm{mmol} \quad \mathrm{Tf}_{2} \mathrm{O} \quad(135 \mu \mathrm{L}), 0.80 \mathrm{mmol} 2,4-$ dichloroquinoline (158 $\mathrm{mg}$ ) and $0.44 \mathrm{mmol}$ azide $2 \mathrm{f}(64.8 \mathrm{mg})$. Purification was performed with column chromatography on silica gel (DMA/DCM gradient, 5:95 to $30: 70$ ) to obtain a mixture of regioisomer.

Yield (orange-brown oil): $84 \%$ (77 mg).

${ }^{1} \mathrm{H}$ NMR $\left(600 \mathrm{MHz}, \mathrm{CDCl}_{3}\right) \delta(\mathrm{ppm}) 9.57(\mathrm{~s}, 0.4 \mathrm{H}), 9.54(\mathrm{~s}, 0.6 \mathrm{H}), 7.39-7.20(\mathrm{~m}, 5 \mathrm{H}), 7.17(\mathrm{~d}, J=7.4 \mathrm{~Hz}, 1 \mathrm{H}), 6.89$ $-6.82(\mathrm{~m}, 1.4 \mathrm{H}), 6.72(\mathrm{~m}, 0.6 \mathrm{H}), 5.55(\mathrm{~s}, 0.4 \mathrm{H}), 5.15(\mathrm{~s}, 0.6 \mathrm{H}), 4.81(\mathrm{~m}, 0.4 \mathrm{H}), 4.79(\mathrm{~m}, 0.6 \mathrm{H}), 4.65(\mathrm{~d}, J=5.6 \mathrm{~Hz}$, $0.6 \mathrm{H}), 4.62(\mathrm{~d}, J=5.5 \mathrm{~Hz}, 0.4 \mathrm{H}), 3.91(\mathrm{~s}, 1 \mathrm{H}), 3.89-3.82(\mathrm{~m}, 1 \mathrm{H}), 3.76(\mathrm{~s}, 4 \mathrm{H}), 3.55(\mathrm{~m}, 0.4 \mathrm{H}), 3.47(\mathrm{~m}, 0.6 \mathrm{H}), 2.14$ $-2.00(\mathrm{~m}, 3 \mathrm{H}), 2.00-1.88(\mathrm{~m}, 1 \mathrm{H})$.

${ }^{13} \mathrm{C}$ NMR (151 MHz, CDCl $) \delta(\mathrm{ppm})\{162.1\}, 162.0,159.5,(155.8), 136.2,(135.1),(131.7), 130.9,130.1$ (2C), (129.7), (129.5), 129.1, 128.7, (128.6), (127.3), 126.6 (2C), 124.0, (120.9), (118.5), 115.4, 110.7, (110.1), (55.8), 55.6, 50.0, (49.9), 49.3, 47.1, 45.5, (45.4), (41.4), 25.4, (25.4), (24.9), 24.8. When distinguishable, the signals of the second minor are reported in brackets.

${ }^{19} \mathrm{~F}$ NMR $\left(565 \mathrm{MHz}, \mathrm{CDCl}_{3}\right) \delta(\mathrm{ppm})-78.2$.

HRMS (ESI) $\mathrm{m} / \mathrm{z}$ calculated for [M-TfO] ${ }^{+} \mathrm{C}_{20} \mathrm{H}_{23} \mathrm{~N}_{2} \mathrm{O}^{+}: 307.1805$, found 307.1806 . 


\section{2. $\alpha$-Amination}

\section{$\underline{N}, \mathrm{~N}$-diethyl-2-(phenethylamino)nonanamide $\mathbf{S} 5$}<smiles>CCC(NCCc1ccccc1)C(=O)N(CC)CC</smiles>

The $\alpha$-aminated amide was prepared using procedure $\mathbf{H}$ with $0.20 \mathrm{mmol}$ amide $\mathbf{S 1}$ (42.7 mg), $0.40 \mathrm{mmol} \mathrm{Tf}_{2} \mathrm{O}(67 \mu \mathrm{L}), 0.40 \mathrm{mmol}$ 2,4-dichloroquinoline (79.2 $\mathrm{mg}$ ) and $0.22 \mathrm{mmol}$ azide $2 \mathrm{a}(32.4 \mathrm{mg})$. Purification was performed with column chromatography on silica gel (DMA/DCM gradient, 5:95 to 20:80).

Yield (orange-brown oil): 39\% (36 mg).

${ }^{1} \mathbf{H}$ NMR $\left(600 \mathrm{MHz}, \mathrm{CDCl}_{3}\right): \delta(\mathrm{ppm}) 7.26(\mathrm{~m}, 2 \mathrm{H}), 7.18(\mathrm{~m}, 3 \mathrm{H}), 3.54(\mathrm{~m}, 1 \mathrm{H}), 3.36(\mathrm{~m}, 2 \mathrm{H}), 3.26-3.13(\mathrm{~m}, 2 \mathrm{H})$, $2.86-2.75(\mathrm{~m}, 2 \mathrm{H}), 2.72(\mathrm{~m}, 1 \mathrm{H}), 2.65-2.56(\mathrm{~m}, 1 \mathrm{H}), 2.27-1.89(\mathrm{br} . \mathrm{s}, 1 \mathrm{H}), 1.57-1.45(\mathrm{~m}, 2 \mathrm{H}), 1.41(\mathrm{~m}, 1 \mathrm{H})$, $1.31-1.22(\mathrm{~m}, 9 \mathrm{H}), 1.20-1.13(\mathrm{t}, J=7.1 \mathrm{~Hz}, 3 \mathrm{H}), 1.11(\mathrm{t}, J=7.1 \mathrm{~Hz}, 3 \mathrm{H}), 0.87(\mathrm{t}, J=7.0 \mathrm{~Hz}, 3 \mathrm{H})$.

${ }^{13} \mathrm{C}$ NMR (151 MHz, CDCl 3 ): $\delta$ (ppm) 174.4, 140.3, 128.8 (2C), 128.4 (2C), 126.1, 58.1, 50.1, 41.4, 40.5, 37.2, $34.5,31.9,29.8,29.3,26.1,22.7,15.0,14.2,13.2$.

HRMS (ESI) m/z calculated for $[\mathrm{M}+\mathrm{H}]^{+} \mathrm{C}_{21} \mathrm{H}_{37} \mathrm{~N}_{2} \mathrm{O}^{+}: 333.2900$, found 333.2901.

$\operatorname{ATR-FTIR}\left(\mathrm{cm}^{-1}\right):$ 2954, 2926, 2854, 1636, 1454, 1427, 1378, 1362, 1259, 1128, 845, 749, 699.

\section{$\underline{N, N \text {-dimethyl-2-(phenethylamino)-4-phenylbutanamide S6 }}$}<smiles>CN(C)C(=O)C(CCc1ccccc1)NCCc1ccccc1</smiles>

The $\alpha$-aminated amide was prepared using procedure $\mathbf{H}$ with $0.20 \mathrm{mmol}$ amide $\mathbf{S 2}$ (35.5 mg), $0.40 \mathrm{mmol} \mathrm{Tf} 2 \mathrm{O}(67 \mu \mathrm{L}), 0.40 \mathrm{mmol}$ 2,4-dichloroquinoline $(79.2 \mathrm{mg}$ ) and $0.22 \mathrm{mmol}$ azide $2 \mathrm{a}(32.4 \mathrm{mg})$. Purification was performed with column chromatography on silica gel (DMA/DCM gradient, 5:95 to 20:80).

Yield (orange-brown oil): 63\% (39 mg).

${ }^{1}{ }_{H}$ NMR $\left(600 \mathrm{MHz}, \mathrm{CDCl}_{3}\right): \delta(\mathrm{ppm}) 7.32-7.24(\mathrm{~m}, 4 \mathrm{H}), 7.24-7.13(\mathrm{~m}, 6 \mathrm{H}), 3.40(\mathrm{dd}, J=7.6,5.4 \mathrm{~Hz}, 1 \mathrm{H}), 2.94$ $(\mathrm{s}, 3 \mathrm{H}), 2.87-2.79(\mathrm{~m}, 2 \mathrm{H}), 2.79-2.76(\mathrm{~m}, 1 \mathrm{H}), 2.75(\mathrm{~s}, 3 \mathrm{H}), 2.75-2.69(\mathrm{~m}, 2 \mathrm{H}), 2.58(\mathrm{~m}, 1 \mathrm{H}), 1.99(\mathrm{br} . \mathrm{s}, 1 \mathrm{H})$, $1.85-1.73(\mathrm{~m}, 2 \mathrm{H})$.

${ }^{13} \mathrm{C}$ NMR $\left(151 \mathrm{MHz}, \mathrm{CDCl}_{3}\right): \delta$ (ppm) 175.0, 141.8, 140.4, 128.9 (2C), 128.7 (2C), $128.5(2 \mathrm{C}), 128.4(2 \mathrm{C}), 126.1$, 126.0, 57.1, 50.2, 37.2, 36.6, 35.8, 35.2, 32.0.

HRMS (ESI) $\mathrm{m} / \mathrm{z}$ calculated for $[\mathrm{M}+\mathrm{H}]^{+} \mathrm{C}_{20} \mathrm{H}_{27} \mathrm{~N}_{2} \mathrm{O}^{+}: 311.2118$, found 311.2117.

$\operatorname{ATR}-\operatorname{FTIR}\left(\mathrm{cm}^{-1}\right):$ 3025, 2927, 2855, 1640, 1495, 1454, 1397, 1258, 1121, 1030, 750, 699 


\subsection{Oxazines and Oxazinone}

\section{2,6-diphenyl-3-(pyrrolidin-1-yl)-2H-1,4-oxazine 8a}<smiles></smiles>

The oxazine was prepared using procedure $\mathbf{H}$ with $0.20 \mathrm{mmol}$ amide $1 \mathrm{a}(37.9 \mathrm{mg})$, $0.40 \mathrm{mmol} \mathrm{Tf}_{2} \mathrm{O}(67 \mu \mathrm{L}), 0.40 \mathrm{mmol}$ 2,4-dichloroquinoline $(79.2 \mathrm{mg})$ and $0.22 \mathrm{mmol}$ azide $7 \mathrm{a}(35.5 \mathrm{mg})$. Purification was performed with column chromatography on silica gel (DMA/DCM gradient, 5:95 to 20:80).

Yield (yellow solid): 90\% (55 mg).

${ }^{1} \mathrm{H}$ NMR $\left(600 \mathrm{MHz}, \mathrm{CDCl}_{3}\right): \delta(\mathrm{ppm}) 7.47-7.43(\mathrm{~m}, 2 \mathrm{H}), 7.41(\mathrm{~m}, 2 \mathrm{H}), 7.36-7.30(\mathrm{~m}, 3 \mathrm{H}), 7.25-7.20(\mathrm{~m}, 2 \mathrm{H})$, $7.12(\mathrm{~m}, 1 \mathrm{H}), 6.78(\mathrm{~s}, 1 \mathrm{H}), 5.87(\mathrm{~s}, 1 \mathrm{H}), 3.80-3.05(\mathrm{~m}, 4 \mathrm{H}), 2.05-1.79(\mathrm{~m}, 4 \mathrm{H})$.

${ }^{13} \mathrm{C}$ NMR (151 MHz, CDCl 3 ): $\delta$ (ppm) 151.7, 135.5, 135.3, 134.4, 129.1, 128.8 (2C), 128.3 (2C), 128.0 (2C), 126.7, $123.5(2 \mathrm{C}), 116.0,71.6,46.5(2 \mathrm{C}), 25.1(2 \mathrm{C})$.

HRMS (ESI) m/z calculated for [M+H] ${ }^{+} \mathrm{C}_{20} \mathrm{H}_{21} \mathrm{~N}_{2} \mathrm{O}^{+}: 305,1648$, found 305,1651 .

ATR-FTIR $\left(\mathrm{cm}^{-1}\right)$ : 3071, 3025, 2962, 2872, 1599, 1567, 1488, 1447, 1342, 1055, 1026, 951, 761, 750, 690, 479.

Mp.: $218-221^{\circ} \mathrm{C}$.

\section{6-(naphthalen-2-yl)-2-phenyl-3-(pyrrolidin-1-yl)-2H-1,4-oxazine 8b}<smiles>c1ccc(C2N=C(N3CCCC3)C(c3ccc4ccccc4c3)O2)cc1</smiles>

The oxazine was prepared using procedure $\mathbf{H}$ with $0.20 \mathrm{mmol}$ amide $1 \mathbf{a}$ (37.9 mg), $0.40 \mathrm{mmol} \mathrm{Tf} \mathrm{O}_{2} \mathrm{O}(67 \mu \mathrm{L}), 0.40 \mathrm{mmol}$ 2,4-dichloroquinoline (79.2 mg) and $0.22 \mathrm{mmol}$ azide $\mathbf{7 b}(46.5 \mathrm{mg})$. Purification was performed with column chromatography on silica gel (DMA/DCM gradient, 5:95 to 20:80).

Yield (yellow solid): 81\% (57 mg).

${ }^{1} \mathrm{H}$ NMR $\left(600 \mathrm{MHz}, \mathrm{CDCl}_{3}\right): \delta(\mathrm{ppm}) 7.88(\mathrm{~s}, 1 \mathrm{H}), 7.78(\mathrm{~d}, J=8.1 \mathrm{~Hz}, 1 \mathrm{H}), 7.72(\mathrm{~d}, J=8.1 \mathrm{~Hz}, 1 \mathrm{H}), 7.66(\mathrm{~d}, J=8.7 \mathrm{~Hz}$, $1 \mathrm{H}), 7.53(\mathrm{dd}, J=8.7,1.4 \mathrm{~Hz}, 1 \mathrm{H}), 7.49(\mathrm{~d}, J=7.2 \mathrm{~Hz}, 2 \mathrm{H}), 7.42(\mathrm{~m}, 1 \mathrm{H}), 7.38-7.28(\mathrm{~m}, 4 \mathrm{H}), 6.94(\mathrm{~s}, 1 \mathrm{H}), 5.93(\mathrm{~s}$, $1 \mathrm{H}), 3.82-3.07(\mathrm{~m}, 4 \mathrm{H}), 2.07-1.81(\mathrm{~m}, 4 \mathrm{H})$.

${ }^{13} \mathrm{C}$ NMR $\left(151 \mathrm{MHz}, \mathrm{CDCl}_{3}\right): \delta$ (ppm) 152.0, 135.5, 135.3, 133.7, 132.5, 131.7, 129.1, 128.8 (2C), 128.1, 127.8 (2C), $127.7,127.6,126.1,125.4,121.8,121.4,116.9,71.7,46.4$ (2C), 24.9 (2C).

HRMS (ESI) $\mathrm{m} / \mathrm{z}$ calculated for $[\mathrm{M}+\mathrm{H}]^{+} \mathrm{C}_{24} \mathrm{H}_{23} \mathrm{~N}_{2} \mathrm{O}^{+}: 355,1805$, found 355,1798 .

ATR-FTIR $\left(\mathrm{cm}^{-1}\right)$ : 3058, 2968, 2869, 1561, 1448, 1338, 1212, 1060, 857, 817, 747, 699, 476.

Mp.: $179-181^{\circ} \mathrm{C}$. 


\section{6-(4-methoxyphenyl)-2-phenyl-3-(pyrrolidin-1-yl)-2H-1,4-oxazine 8c}<smiles>COc1ccc(C2=NN=C(N3CCCC3)C(c3ccccc3)O2)cc1</smiles>

The oxazine was prepared using procedure $\mathbf{H}$ with $0.20 \mathrm{mmol}$ amide $1 \mathrm{a}$ (37.9 mg), $0.40 \mathrm{mmol} \mathrm{Tf} \mathrm{O}_{2}(67 \mu \mathrm{L}), 0.40 \mathrm{mmol}$ 2,4-dichloroquinoline (79.2 mg) and $0.22 \mathrm{mmol}$ azide $7 \mathrm{c}(42.1 \mathrm{mg})$. Purification was performed with column chromatography on silica gel (DMA/DCM gradient, 5:95 to 20:80).

Yield (yellow solid): 56\% (37 mg).

${ }^{1} \mathrm{H}$ NMR $\left(600 \mathrm{MHz}, \mathrm{CDCl}_{3}\right): \delta 7.45(\mathrm{~d}, J=6.7 \mathrm{~Hz}, 2 \mathrm{H}), 7.35-7.29(\mathrm{~m}, 5 \mathrm{H}), 6.82-6.74(\mathrm{~m}, 2 \mathrm{H}), 6.63(\mathrm{~s}, 1 \mathrm{H}), 5.85(\mathrm{~s}$, $1 \mathrm{H}), 3.75(\mathrm{~s}, 3 \mathrm{H}), 3.37(\mathrm{~m}, 4 \mathrm{H}), 2.03-1.80(\mathrm{~m}, 4 \mathrm{H})$.

${ }^{13} \mathrm{C}$ NMR $(151 \mathrm{MHz}, \mathrm{CDCl})$ ): $\delta$ (ppm) 158.8, 151.3, 135.6, 135.4, 129.1, 128.8 (2C), 127.9 (2C), 127.2, 125.0 (2C), $114.4,113.8(2 \mathrm{C}), 71.6,55.4,46.4(2 \mathrm{C}), 24.9(2 \mathrm{C})$.

HRMS (ESI) $\mathrm{m} / \mathrm{z}$ calculated for $[\mathrm{M}+\mathrm{H}]^{+} \mathrm{C}_{21} \mathrm{H}_{23} \mathrm{~N}_{2} \mathrm{O}_{2}{ }^{+}: 335,1754$, found 335,1758 .

ATR-FTIR (cm $\left.{ }^{-1}\right)$ : 3061, 2966, 2870, 1606, 1576, 1509, 1458, 1446, 1339, 1248, 1175, 1059, 1031, 829, 699.

Mp.: $159-162{ }^{\circ} \mathrm{C}$.

6-(4-nitrophenyl)-2-phenyl-3-(pyrrolidin-1-yl)-2H-1,4-oxazine 8d<smiles></smiles>

The oxazine was prepared using procedure $\mathbf{H}$ with $0.2 \mathrm{mmol}$ amide $1 \mathrm{a}(37.9 \mathrm{mg})$, $0.4 \mathrm{mmol} \mathrm{Tf} 2 \mathrm{O}(67.3 \mu \mathrm{L}), 0.4 \mathrm{mmol}$ 2,4-dichloroquinoline $(79.2 \mathrm{mg}$ ) and 0.22 mmol azide 7d $(45.4 \mathrm{mg})$. Purification was performed with column chromatography on silica gel (DMA/DCM gradient, 5:95 to 20:80).

Yield (red solid): 81\% (57 mg).

${ }^{1} \mathrm{H}$ NMR $\left(600 \mathrm{MHz}, \mathrm{CDCl}_{3}\right): \delta(\mathrm{ppm}) 8.09-7.99(\mathrm{~m}, 2 \mathrm{H}), 7.51-7.46(\mathrm{~m}, 2 \mathrm{H}), 7.41-7.38(\mathrm{~m}, 2 \mathrm{H}), 7.36-7.32(\mathrm{~m}$, $3 \mathrm{H}), 7.01(\mathrm{~s}, 1 \mathrm{H}), 5.88(\mathrm{~s}, 1 \mathrm{H}), 3.70(\mathrm{~m}, 2 \mathrm{H}), 3.53(\mathrm{~m}, 1 \mathrm{H}), 3.19(\mathrm{~m}, 1 \mathrm{H}), 2.04-1.84(\mathrm{~m}, 4 \mathrm{H})$.

${ }^{13} \mathrm{C}$ NMR $\left(151 \mathrm{MHz}, \mathrm{CDCl}_{3}\right): \delta$ (ppm) 152.9, 145.6, 140.8, 134.5, 133.6, 129.5, 129.0 (2C), 127.8 (2C), 123.9 (2C), $122.7(2 \mathrm{C}), 120.8,71.6,47.1,46.5,25.9,24.5$.

HRMS (ESI) $\mathrm{m} / \mathrm{z}$ calculated for $[\mathrm{M}+\mathrm{H}]^{+} \mathrm{C}_{20} \mathrm{H}_{20} \mathrm{~N}_{3} \mathrm{O}_{3}{ }^{+}: 350,1499$, found 350,1500 .

ATR-FTIR $\left(\mathrm{cm}^{-1}\right)$ : 3061, 2960, 2878, 1559, 1503, 1460, 1333, 1257, 1182, 1112, 1058, 947, 838, 750, 694.

Mp.: $187-190^{\circ} \mathrm{C}$. 
<smiles>CC(C)(C)Oc1ncnc(C2CCCC2)n1</smiles>

The oxazine was prepared using procedure $\mathbf{H}$ with $0.20 \mathrm{mmol}$ amide $1 \mathrm{a}(37.9 \mathrm{mg})$, $0.40 \mathrm{mmol} \mathrm{Tf} 2 \mathrm{O}(67 \mu \mathrm{L}), 0.40 \mathrm{mmol}$ 2,4-dichloroquinoline $(79.2 \mathrm{mg}$ ) and $0.22 \mathrm{mmol}$ azide 7 e $(31.1 \mathrm{mg})$. Purification was performed with column chromatography on silica gel (DMA/DCM gradient, 5:95 to 20:80).

Yield (yellow oil): $88 \%$ (50 mg).

${ }^{1} \mathrm{H}$ NMR $\left.\left(600 \mathrm{MHz}, \mathrm{CDCl}_{3}\right): \delta 8 \mathrm{ppm}\right) 7.39-7.31(\mathrm{~m}, 5 \mathrm{H}), 5.99(\mathrm{~s}, 1 \mathrm{H}), 5.69(\mathrm{~s}, 1 \mathrm{H}), 3.50(\mathrm{~m}, 4 \mathrm{H}), 2.01-1.69(\mathrm{~m}$, $4 \mathrm{H}), 0.83(\mathrm{~s}, 9 \mathrm{H})$.

$\left.{ }^{13} \mathrm{C} \mathrm{NMR} \mathrm{(151} \mathrm{MHz,} \mathrm{CDCl} 3\right): \delta(p p m)$ 150.5, 145.3, 134.2, 129.3, 128.7 (2C), 128.6 (2C), 109.8, 71.6, 46.6 (2C), 33.0, $27.3(3 \mathrm{C}), 25.0(2 \mathrm{C})$.

HRMS (ESI) $\mathrm{m} / \mathrm{z}$ calculated for $[\mathrm{M}+\mathrm{H}]^{+} \mathrm{C}_{18} \mathrm{H}_{25} \mathrm{~N}_{2} \mathrm{O}^{+}: 285,1861$, found 285.1866 .

ATR-FTIR $\left(\mathrm{cm}^{-1}\right)$ : 2963, 2869, 1659, 1623, 1582, 1447, 1341, 1358, 1341, 1158, 1030, 762, 699, 638, 518.

\section{5,6-dimethyl-2-phenyl-3-(pyrrolidin-1-yl)-2H-1,4-oxazine 8f}<smiles>Cc1nc(N2CCCC2)c(-c2ccccc2)o1</smiles>

The oxazine was prepared using procedure $\mathbf{H}$ with $0.20 \mathrm{mmol}$ amide $1 \mathrm{1a}(37.9 \mathrm{mg})$, $0.40 \mathrm{mmol} \mathrm{Tf}_{2} \mathrm{O}(67 \mu \mathrm{L}), 0.40 \mathrm{mmol}$ 2,4-dichloroquinoline $(79.2 \mathrm{mg})$ and $0.22 \mathrm{mmol}$ azide 7f $(24.9 \mathrm{mg})$. Purification was performed with column chromatography on silica gel (DMA/DCM gradient, 5:95 to 20:80).

Yield (yellow oil): $59 \%$ (30 mg).

${ }^{1} \mathrm{H} \mathrm{NMR}\left(400 \mathrm{MHz}, \mathrm{CDCl}_{3}\right): \delta(\mathrm{ppm}) 7.42-7.28(\mathrm{~m}, 5 \mathrm{H}), 5.64(\mathrm{~s}, 1 \mathrm{H}), 3.51(\mathrm{~m}, 4 \mathrm{H}), 2.04-1.86(\mathrm{~m}, 4 \mathrm{H}), 1.85$ (app.d, $J=0.8 \mathrm{~Hz}, 3 \mathrm{H}$ ), 1.66 (app.d, $J=0.8 \mathrm{~Hz}, 3 \mathrm{H}$ ).

${ }^{13} \mathrm{C}$ NMR (151 MHz, DMSO): $\delta$ (ppm) 150.2, 136.3, 128.7, 128.6 (2C), 128.1, 127.2 (2C), 118.7, 69.9, 46.6 (2C), $24.7(2 \mathrm{C}), 17.1,15.4$.

HRMS (ESI) $\mathrm{m} / \mathrm{z}$ calculated for $[\mathrm{M}+\mathrm{H}]^{+} \mathrm{C}_{16} \mathrm{H}_{21} \mathrm{~N}_{2} \mathrm{O}^{+}: 257.1648$, found 257.1650 .

ATR-FTIR $\left(\mathrm{cm}^{-1}\right)$ : 2950, 2920, 2869, 1725, 1659, 1585, 1444, 1340, 1279, 1156, 1140, 1031, 752, 737, 638.

\section{2-phenyl-3-(pyrrolidin-1-yl)-5,6,7,8-tetrahydro-2H-benzo[b][1,4]oxazine 8g}<smiles></smiles>

The oxazine was prepared using procedure $\mathbf{H}$ with $0.20 \mathrm{mmol}$ amide $1 \mathrm{a}(37.9 \mathrm{mg})$, $0.40 \mathrm{mmol} \mathrm{Tf} \mathrm{T}_{2} \mathrm{O}(67 \mu \mathrm{L}), 0.40 \mathrm{mmol}$ 2,4-dichloroquinoline $(79.2 \mathrm{mg}$ ) and $0.22 \mathrm{mmol}$ azide $7 \mathrm{~g}$ (30.6 mg). Purification was performed with column chromatography on silica gel (DMA/DCM gradient, 5:95 to 20:80).

Yield (yellow oil): $70 \%$ (40 mg).

${ }^{1} \mathrm{H}$ NMR $\left(600 \mathrm{MHz}, \mathrm{CDCl}_{3}\right): \delta(\mathrm{ppm}) 7.41-7.28(\mathrm{~m}, 5 \mathrm{H}), 5.59(\mathrm{~s}, 1 \mathrm{H}), 3.37(\mathrm{~m}, 4 \mathrm{H}), 2.35-2.25(\mathrm{~m}, 1 \mathrm{H}), 2.15(\mathrm{~m}$, $1 \mathrm{H}), 2.06(\mathrm{~m}, 1 \mathrm{H}), 1.97-1.88(\mathrm{~m}, 2 \mathrm{H}), 1.87-1.77(\mathrm{~m}, 3 \mathrm{H}), 1.70-1.64(\mathrm{~m}, 1 \mathrm{H}), 1.61-1.47(\mathrm{~m}, 2 \mathrm{H}), 1.42-1.31$ $(\mathrm{m}, 1 \mathrm{H})$.

${ }^{13} \mathrm{C} \mathrm{NMR}\left(151 \mathrm{MHz}, \mathrm{CDCl}_{3}\right): \delta$ (ppm) 150.9, 136.0, 131.4, 128.9, 128.7 (2C), 127.7 (2C), 121.6, 71.5, 46.7 (2C), 28.2, 26.5, $25.1(2 \mathrm{C}), 23.2,23.0$. 
HRMS (ESI) $\mathrm{m} / \mathrm{z}$ calculated for $[\mathrm{M}+\mathrm{H}]^{+} \mathrm{C}_{18} \mathrm{H}_{23} \mathrm{~N}_{2} \mathrm{O}^{+}: 283.1805$, found 283.1808.

ATR-FTIR (cm $\left.{ }^{-1}\right):$ 2928, 2857, 1656, 1578, 1491, 1442, 1341, 1246, 1145, 1029, 913, 735, 699, 637, 517.

2,7-diphenyl-3-(pyrrolidin-1-yl)-2,5-dihydro-1,4-oxazepin-4-ium-trifluoromethanesulfonate 8h<smiles>C1=C(c2ccccc2)OC(c2ccccc2)C(N2CCCC2)=NC1</smiles>

The oxazine was prepared using procedure $\mathbf{H}$ with $0.20 \mathrm{mmol}$ amide 1 a $(37.9 \mathrm{mg})$, $0.40 \mathrm{mmol} \mathrm{Tf} 2 \mathrm{O}(67 \mu \mathrm{L}), 0.40 \mathrm{mmol}$ 2,4-dichloroquinoline (79.2 $\mathrm{mg}$ ) and $0.22 \mathrm{mmol}$ azide $7 \mathrm{~h}$ (38.5 mg). Purification was performed with column chromatography on silica gel (DMA/DCM gradient, 5:95 to 30:70).

Yield (yellow solid): $72 \%$ (67 mg).

${ }^{1} \mathrm{H}$ NMR $\left(600 \mathrm{MHz}, \mathrm{CDCl}_{3}\right): \delta(\mathrm{ppm}) 9.48$ (br.s, $\left.1 \mathrm{H}\right), 7.54(\mathrm{~m}, 2 \mathrm{H}), 7.47(\mathrm{~m}, 3 \mathrm{H}), 7.42-7.34(\mathrm{~m}, 5 \mathrm{H}), 6.15(\mathrm{~s}, 1 \mathrm{H})$, $5.78(\mathrm{dd}, J=6.9,4.4 \mathrm{~Hz}, 1 \mathrm{H}), 4.22(\mathrm{dd}, J=16.9,6.9 \mathrm{~Hz}, 1 \mathrm{H}), 4.11(\mathrm{dd}, J=16.9,4.4 \mathrm{~Hz}, 1 \mathrm{H}), 3.83(\mathrm{~m}, 2 \mathrm{H}), 3.66(\mathrm{~m}$, $1 \mathrm{H}), 3.28(\mathrm{~m}, 1 \mathrm{H}), 2.15-1.95(\mathrm{~m}, 4 \mathrm{H})$.

${ }^{13} \mathrm{C}$ NMR $\left(151 \mathrm{MHz}, \mathrm{CDCl}_{3}\right): \delta$ (ppm) 161.5, 154.7, 133.9, 132.5, 130.5, $130.0(2 \mathrm{C}), 129.7,128.8(2 \mathrm{C}), 126.1(2 \mathrm{C})$, $125.1(2 \mathrm{C}), 105.0,77.5,50.6,50.1,40.6,25.7,24.6$.

${ }^{19} \mathrm{~F}$ NMR $\left(565 \mathrm{MHz}, \mathrm{CDCl}_{3}\right): \delta(\mathrm{ppm})-78.2$.

HRMS (ESI) m/z calculated for [M-TfO] ${ }^{+} \mathrm{C}_{21} \mathrm{H}_{23} \mathrm{~N}_{2} \mathrm{O}^{+}: 319.1805$, found 319.1804.

$\operatorname{ATR-FTIR}\left(\mathrm{cm}^{-1}\right):$ 3218, 3080, 2956, 1653, 1452, 1246, 1159, 1030, 757, 697, 574, 518

Mp.: $217-219^{\circ} \mathrm{C}$.

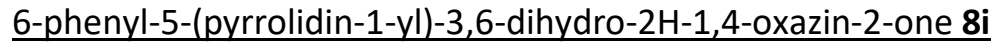<smiles>O=C1CN=C(N2CCCC2)C(c2ccccc2)O1</smiles>

The oxazinone was prepared using procedure $\mathbf{H}$ with $0.20 \mathrm{mmol}$ amide $\mathbf{1 a}(37.9 \mathrm{mg})$, $0.40 \mathrm{mmol} \mathrm{Tf}_{2} \mathrm{O}(67 \mu \mathrm{L}), 0.40 \mathrm{mmol}$ 2,4-dichloroquinoline $(79.2 \mathrm{mg})$ and $0.22 \mathrm{mmol}$ azide $7 \mathbf{i}(37.6 \mathrm{mg})$. Due to its high sensitivity, the purification was performed by dissolving the crude product in ACN and washing with heptane (5x).

Yield (yellow oil): $70 \%$ (34 mg).

${ }^{1} \mathbf{H}$ NMR $\left(600 \mathrm{MHz}, \mathrm{CDCl}_{3}\right): \delta(\mathrm{ppm}) 7.41(\mathrm{~m}, 3 \mathrm{H}), 7.37-7.32(\mathrm{~m}, 2 \mathrm{H}), 6.07(\mathrm{~s}, 1 \mathrm{H}), 4.30(\mathrm{~d}, J=19.9 \mathrm{~Hz}, 1 \mathrm{H}), 3.81$ $(\mathrm{d}, J=19.9 \mathrm{~Hz}, 1 \mathrm{H}), 3.41(\mathrm{~m}, 4 \mathrm{H}), 2.01-1.84(\mathrm{~m}, 4 \mathrm{H})$.

${ }^{13} \mathrm{C}$ NMR (151 MHz, $\left.\mathrm{CDCl}_{3}\right): \delta(\mathrm{ppm}) 170.1,156.1,133.4,129.9,129.6(2 \mathrm{C}), 127.4(2 \mathrm{C}), 77.3,49.0,46.9(2 \mathrm{C})$, $25.4(2 \mathrm{C})$.

HRMS (ESI) $\mathrm{m} / \mathrm{z}$ calculated for $[\mathrm{M}+\mathrm{H}]^{+} \mathrm{C}_{14} \mathrm{H}_{17} \mathrm{~N}_{2} \mathrm{O}_{2}{ }^{+}: 245.1285$, found 245.1286.

$\operatorname{ATR}-\mathrm{FTIR}\left(\mathrm{cm}^{-1}\right)$ : 2968, 2870, 2106, 1744, 1620, 1446, 1312, 1236, 1186, 1028, 911, 739, 699, 637, 517. 


\section{Derivatisation of Amidines}

\subsection{Hydrolysis}

\section{General Procedure I}

A mixture of amidine ( $0.20 \mathrm{mmol}, 1$ eq.), THF ( $1 \mathrm{~mL}), \mathrm{NaOH}(1 \mathrm{~mL}, 5 \mathrm{M}, 5$ eq.) and TPA-OH $(4 \mu \mathrm{L}$, $0.02 \mathrm{mmol}, 0.1 \mathrm{eq}$ ) were stirred under reflux for $15 \mathrm{~h}$. Purification was performed via addition of $\mathrm{NH}_{4} \mathrm{Cl}$ and extraction with (3x) DCM. The combined organic layers were dried over anhydrous $\mathrm{Na}_{2} \mathrm{SO}_{4}$ and concentrated under reduced pressure.

\section{1-phenyl-1,3,4,5-tetrahydro-2H-benzo[d]azepin-2-one 4a}<smiles>O=C1NCCc2ccccc2C1c1ccccc1</smiles>

The benzoazepinone was obtained using procedure I with amidine $\mathbf{3 a}(88.1 \mathrm{mg})$. The crude product was further purified by flash chromatography on silica gel (DMA/DCM gradient, 0:100 to 10:90).

Yield (yellow oil): $63 \%$ (30 mg).

${ }^{1} \mathrm{H}$ NMR $\left(600 \mathrm{MHz}, \mathrm{CDCl}_{3}\right): \delta(\mathrm{ppm}) 7.28(\mathrm{~m}, 3 \mathrm{H}), 7.27-7.24(\mathrm{~m}, 1 \mathrm{H}), 7.23(\mathrm{~m}, 1 \mathrm{H}), 7.20(\mathrm{~m}, 1 \mathrm{H}), 7.18-7.16(\mathrm{~m}$, $1 \mathrm{H}), 7.12-7.08(\mathrm{~m}, 2 \mathrm{H}), 6.71$ (br.s, $1 \mathrm{H}), 5.15(\mathrm{~s}, 1 \mathrm{H}), 3.37$ (ddd, $J=19.2,9.6,4.7 \mathrm{~Hz}, 1 \mathrm{H}), 3.12-3.05(\mathrm{~m}, 1 \mathrm{H})$, $3.04-2.95(\mathrm{~m}, 2 \mathrm{H})$.

${ }^{13} \mathrm{C}$ NMR $\left(151 \mathrm{MHz}, \mathrm{CDCl}_{3}\right): \delta$ (ppm) 175.1, 139.8, 137.8, 133.5, 132.9, 131.2, 128.8 (2C), 127.9, 127.1, 126.9, $126.9(2 \mathrm{C}), 60.5,39.7,33.8$.

HRMS (ESI) $\mathrm{m} / \mathrm{z}$ calculated for $[\mathrm{M}+\mathrm{H}]^{+} \mathrm{C}_{16} \mathrm{H}_{16} \mathrm{NO}^{+}: 238.1226$, found 238.1228 .

ATR-FTIR $\left(\mathrm{cm}^{-1}\right)$ : 3199, 3081, 2935, 1666, 1493, 1406, 1342, 806, 759, 749, 706.

\section{7-methoxy-4-phenyl-1,4-dihydroisoquinolin-3(2H)-one 4ba}<smiles>COc1ccc2c(c1)CNC(=O)C2c1ccccc1</smiles>

The isolated, hydrolysed regioisomer was obtained using procedure I with isomeremixture $3 \mathrm{~m}$. The crude product was further purified by flash chromatography on silica gel (DMA/DCM gradient, 0:100 to 10:90).

Yield (brown amorphous solid): $17 \%$ (8.6 mg).

${ }^{1} \mathrm{H}$ NMR $\left(600 \mathrm{MHz}, \mathrm{CDCl}_{3}\right): \delta(\mathrm{ppm}) 7.28(\mathrm{~m}, 2 \mathrm{H}), 7.23(\mathrm{~m}, 1 \mathrm{H}), 7.19-7.13(\mathrm{~m}, 2 \mathrm{H}), 7.03(\mathrm{~d}, J=8.5 \mathrm{~Hz}, 1 \mathrm{H}), 6.84$ (dd, $J=8.5,2.5 \mathrm{~Hz}, 1 \mathrm{H}), 6.75(\mathrm{~d}, J=2.5 \mathrm{~Hz}, 1 \mathrm{H}), 6.71(\mathrm{br} . \mathrm{s}, 1 \mathrm{H}), 4.76(\mathrm{~s}, 1 \mathrm{H}), 4.54(\mathrm{~d}, J=15.8 \mathrm{~Hz}, 1 \mathrm{H}), 4.35$ (dd, $J$ $=15.8,4.1 \mathrm{~Hz}, 1 \mathrm{H}), 3.82(\mathrm{~s}, 3 \mathrm{H})$.

${ }^{13} \mathrm{C}$ NMR (151 MHz, CDCl $\left.)_{3}\right): \delta$ (ppm) 173.0, 158.8, 139.0, 132.9, 130.1, 128.8 (2C), 128.1 (2C), 127.4, 127.3, 113.9, $110.7,55.5,51.8,45.4$.

HRMS (ESI) $\mathrm{m} / \mathrm{z}$ calculated for $[\mathrm{M}+\mathrm{H}]^{+} \mathrm{C}_{16} \mathrm{H}_{16} \mathrm{NO}_{2}{ }^{+}: 254.1176$, found 254.1177.

ATR-FTIR $\left(\mathrm{cm}^{-1}\right):$ 3219, 3061, 2930, 2838, 1668, 1613, 1493, 1324, 1272, 1240, 1035, 738, 700. 


\section{6-methoxy-4-phenyl-1,4-dihydroisoquinolin-3(2H)-one 4bb}

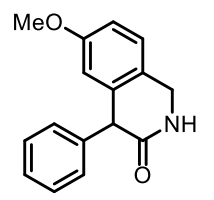

The isolated, hydrolysed regioisomere was obtained using procedure I with isomeremixture $\mathbf{3 m}$. The crude product was further purified by flash chromatography on silica gel (DMA/DCM gradient, 0:100 to 10:90).

Yield (white amorphous solid): 7 \% (3.5 mg).

${ }^{1} \mathrm{H}$ NMR $\left(600 \mathrm{MHz}, \mathrm{CDCl}_{3}\right): \delta(\mathrm{ppm}) 7.28(\mathrm{~m}, 1 \mathrm{H}), 7.24(\mathrm{~m}, 2 \mathrm{H}), 7.21(\mathrm{~m}, 3 \mathrm{H}), 6.84(\mathrm{~m}, 2 \mathrm{H}), 6.27(\mathrm{br} . \mathrm{s}, 1 \mathrm{H}), 5.17(\mathrm{~s}$, $1 \mathrm{H}), 4.61(\mathrm{~d}, J=15.7 \mathrm{~Hz}, 1 \mathrm{H}), 4.32(\mathrm{dd}, J=15.7,4.8 \mathrm{~Hz}, 1 \mathrm{H}), 3.75(\mathrm{~s}, 3 \mathrm{H})$.

${ }^{13} \mathrm{C}$ NMR $\left(151 \mathrm{MHz}, \mathrm{CDCl}_{3}\right): \delta$ (ppm) 172.8, 156.9, 138.3, 133.3, 128.7 (2C), 128.4, 127.7 (2C), 127.2, 124.1, 117.7, $109.6,55.7,46.6,45.3$.

HRMS (ESI) $\mathrm{m} / \mathrm{z}$ calculated for $[\mathrm{M}+\mathrm{H}]^{+} \mathrm{C}_{16} \mathrm{H}_{16} \mathrm{NO}_{2}{ }^{+}: 254.1176$, found 254.1177 .

ATR-FTIR $\left(\mathrm{cm}^{-1}\right)$ : 3210, 3059, 2931, 2839, 1671, 1595, 1471, 1256, 1072, 782, 745, 700.

\subsection{Deprotonation}

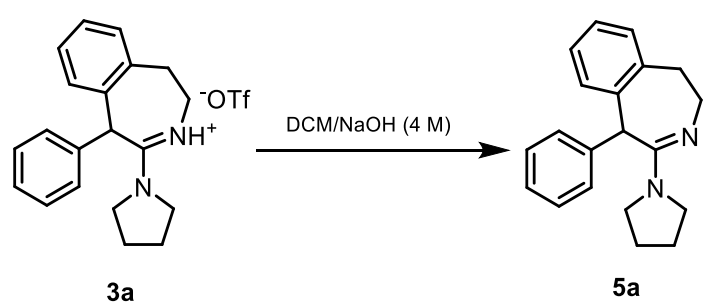

5-phenyl-4-(pyrrolidin-1-yl)-2,5-dihydro-1H-benzo[d]azepine 5a

The free base of 3a was obtained by extraction $\mathrm{DCM} / \mathrm{NaOH} 4 \mathrm{M}$. The organic layer was dried over $\mathrm{K}_{2} \mathrm{CO}_{3}$, filtered and evaporated.

Yield (brown oil): 99\% (58 mg).

${ }^{1} \mathrm{H}$ NMR $\left(600 \mathrm{MHz}, \mathrm{CDCl}_{3}\right): \delta(\mathrm{ppm}) 7.26(\mathrm{~m}, 3 \mathrm{H}), 7.23-7.16(\mathrm{~m}, 3 \mathrm{H}), 7.12(\mathrm{~d}, J=7.2 \mathrm{~Hz}, 1 \mathrm{H}), 7.04(\mathrm{~d}, J=8.1 \mathrm{~Hz}$, $2 \mathrm{H}), 5.24(\mathrm{~s}, 1 \mathrm{H}), 3.63-3.52(\mathrm{~m}, 5 \mathrm{H}), 3.32(\mathrm{ddd}, J=13.8,4.4,4.4 \mathrm{~Hz}, 1 \mathrm{H}), 2.99-2.92(\mathrm{~m}, 2 \mathrm{H}), 1.99-1.91(\mathrm{~m}, 4 \mathrm{H})$.

${ }^{13} \mathrm{C}$ NMR (151 MHz, CDCl $)$ : $\delta$ (ppm) 162.1, 142.2, 140.6, 134.5, 132.6, 131.7, 128.6 (2C), 127.6, 126.4 (2C), 126.3, $125.9,53.2,47.5(2 \mathrm{C}), 45.1,33.9,25.7$.

HRMS (ESI) $\mathrm{m} / \mathrm{z}$ calculated for $[\mathrm{M}+\mathrm{H}]^{+} \mathrm{C}_{20} \mathrm{H}_{23} \mathrm{~N}_{2}{ }^{+}: 291.1856$, found 291.1858 .

ATR-FTIR (cm ${ }^{-1}$ ): 3058, 3022, 2921, 2867, 1611, 1493, 1421, 1362, 1336, 1263, 950, 909, 748, 721, 696, 636, 569. 


\subsection{Methylation}

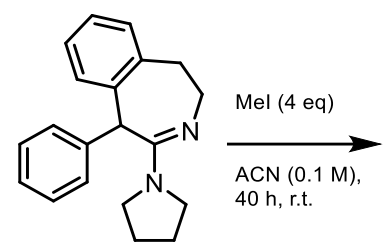

5 a<smiles>C[N+]1(F)CCc2ccccc2C1c1ccccc1</smiles>

$6 a$

3-methyl-5-phenyl-4-(pyrrolidin-1-yl)-2,5-dihydro-1H-benzo[d]azepin-3-ium 6a

The methylated product $6 \mathrm{a}$ was obtained by dropwise addition of Mel $(50 \mu \mathrm{L}, 0.80 \mathrm{mmol}, 3.0 \mathrm{eq})$ to a mixture of $5 \mathrm{a}(47.5 \mathrm{mg}, 0.20 \mathrm{mmol}, 1.0 \mathrm{eq})$ in ACN (0.1 M). The reaction progress was followed by LCMS. After 40h, the mixture was diluted with $\mathrm{MeOH}$ and concentrated under reduced pressure. Purification was performed by flash column chromatography on silica gel (DMA/DCM gradient, 0:100 to $20: 80)$.

Yield (brown-yellow oil): $56 \%$ (48 mg); 61\% brsm

${ }^{1} \mathrm{H}$ NMR $\left(600 \mathrm{MHz}, \mathrm{CDCl}_{3}\right): \delta(\mathrm{ppm}) 7.35(\mathrm{~m}, 3 \mathrm{H}), 7.32-7.26(\mathrm{~m}, 2 \mathrm{H}), 7.25-7.20(\mathrm{~m}, 2 \mathrm{H}), 7.03(\mathrm{~d}, J=7.8 \mathrm{~Hz}, 2 \mathrm{H})$, $5.67(\mathrm{~s}, 1 \mathrm{H}), 4.30(\mathrm{~m}, 1 \mathrm{H}), 4.18-3.88(\mathrm{~m}, 4 \mathrm{H}), 3.58(\mathrm{~s}, 3 \mathrm{H}), 3.39-3.29(\mathrm{~m}, 1 \mathrm{H}), 3.20(\mathrm{~m}, 1 \mathrm{H}), 3.01(\mathrm{~m}, 1 \mathrm{H}), 2.33$ (m, 1H), $2.12(\mathrm{~m}, 3 \mathrm{H})$.

${ }^{13} \mathrm{C}$ NMR $\left(151 \mathrm{MHz}, \mathrm{CDCl}_{3}\right): \delta$ (ppm) 170.0, 137.6, 136.8, 133.1, 131.8, 129.8 (2C), 129.7, 129.5, 128.2, 127.7, $126.2(2 \mathrm{C}), 55.8,53.3,51.4,51.3,45.2,31.0,25.4,25.3$.

HRMS (ESI) $\mathrm{m} / \mathrm{z}$ calculated for $[\mathrm{M}]^{+} \mathrm{C}_{21} \mathrm{H}_{25} \mathrm{~N}_{2}{ }^{+}: 305.2012$, found 305.2014.

ATR-FTIR $\left(\mathrm{cm}^{-1}\right):$ 3144, 3042, 2954, 2924, 2874, 1726, 1634, 1494, 1447, 1338, 1268, 1159, 759, 731, 701 


\section{X-ray Analysis}

The X-ray intensity data were measured on Bruker D8 Venture diffractometer equipped with multilayer monochromators, Mo K/ $\alpha$ INCOATEC micro focus sealed tubes and Oxford system. The structure was solved by direct methods and refined by full-matrix least-squares techniques. Non-hydrogen atoms were refined with anisotropic displacement parameters. Hydrogen atoms were inserted at calculated positions and refined with riding model. The following software was used: Bruker SAINT software package $e^{20}$ using a narrow-frame algorithm for frame integration, $S A D A B S^{21}$ for absorption correction, OLEX $2^{22}$ for structure solution, refinement, molecular diagrams and graphical user-interface, Shelxle ${ }^{23}$ for refinement and graphical user-interface SHELXS-2015 ${ }^{24}$ for structure solution, SHELXL-2015 ${ }^{25}$ for refinement, Platon $^{26}$ for symmetry check and $\pi-\pi$ Interactions. Experimental data and CCDC-Codes (Available online: http://www.ccdc.cam.ac.uk/conts/retrieving.html) can be found in Table 1. Crystal data, data collection parameters and structure refinement details are given in Tables 2 to 5. Crystal structures and Packing views are visualized in Figures 1 to 4.

Table S3 Experimental parameter and CCDC-Codes.

\begin{tabular}{|l|c|c|c|c|c|c|c|c|}
\hline \multicolumn{1}{|c|}{ Sample } & Machine & Source & Temp. & $\begin{array}{c}\text { Detector } \\
\text { Distance }\end{array}$ & $\begin{array}{c}\text { Time/ } \\
\text { Frame }\end{array}$ & \#Frames & $\begin{array}{c}\text { Frame } \\
\text { width }\end{array}$ & CCDC \\
\hline & Bruker & & {$[\mathrm{K}]$} & {$[\mathrm{mm}]$} & {$[\mathrm{s}]$} & & {$\left[^{\circ}\right]$} & \\
\hline $\mathbf{8 a}$ & D8 & Mo & 120 & 40 & 15 & 1176 & 0.500 & 1983046 \\
\hline 3a & D8 & Mo & 100 & 40 & 10 & 360 & 0.500 & 1983045 \\
\hline
\end{tabular}

\footnotetext{
${ }^{20}$ Bruker SAINT v8.38A/B \& SAINT v7.56/7.68A Copyright (C) 2005-2019 Bruker AXS.

${ }^{21}$ Krause, L.; Herbst-Irmer, R.; Sheldrick G. M.; Stalke D. J. Appl. Cryst., 2015, 48, 3-10.

${ }^{22}$ Dolomanov, O.V.; Bourhis, L.J.; Gildea, R.J; Howard, J.A.K.; Puschmann, H. OLEX2, J. Appl. Cryst. 2009, 42, $339-341$.

${ }^{23}$ Huebschle, C. B.; Sheldrick G. M.; Dittrich B.; ShelXle: a Qt graphical user interface for SHELXL, J. Appl. Cryst. 2011, 44 , 1281-1284.

${ }^{24}$ Sheldrick, G. M. Acta Cryst. 2008, A64, 112-122.

${ }^{25}$ Sheldrick, G. M. Acta Cryst. 2015, C71, 3-8.

${ }^{26}$ A. L. Spek, Acta Cryst. 2009, D65, 148-155.
} 


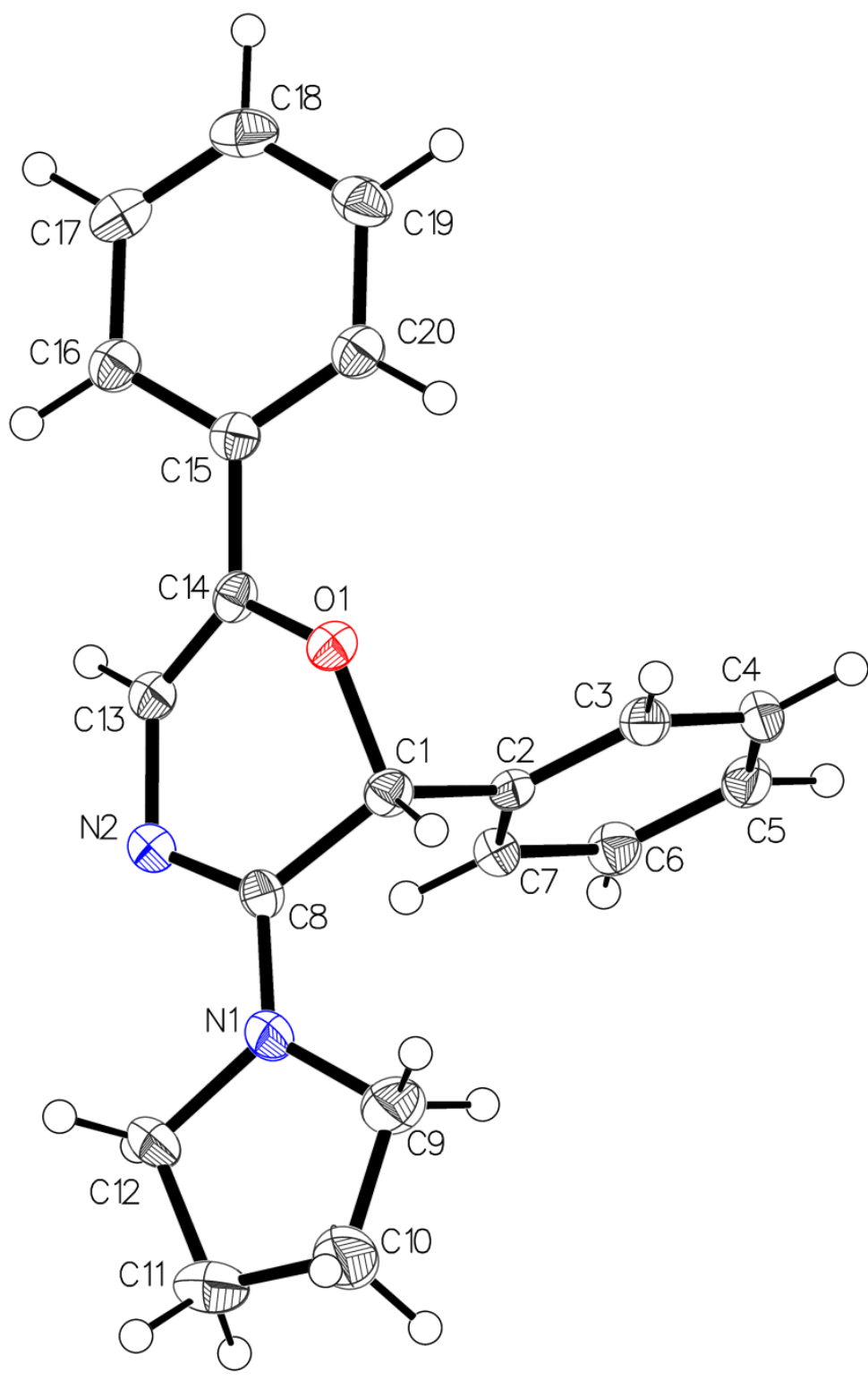

Figure S1 Crystal structure, drawn with $50 \%$ displacement ellipsoid. The bond precision for C-C single bonds is $0.0032 \AA$. 


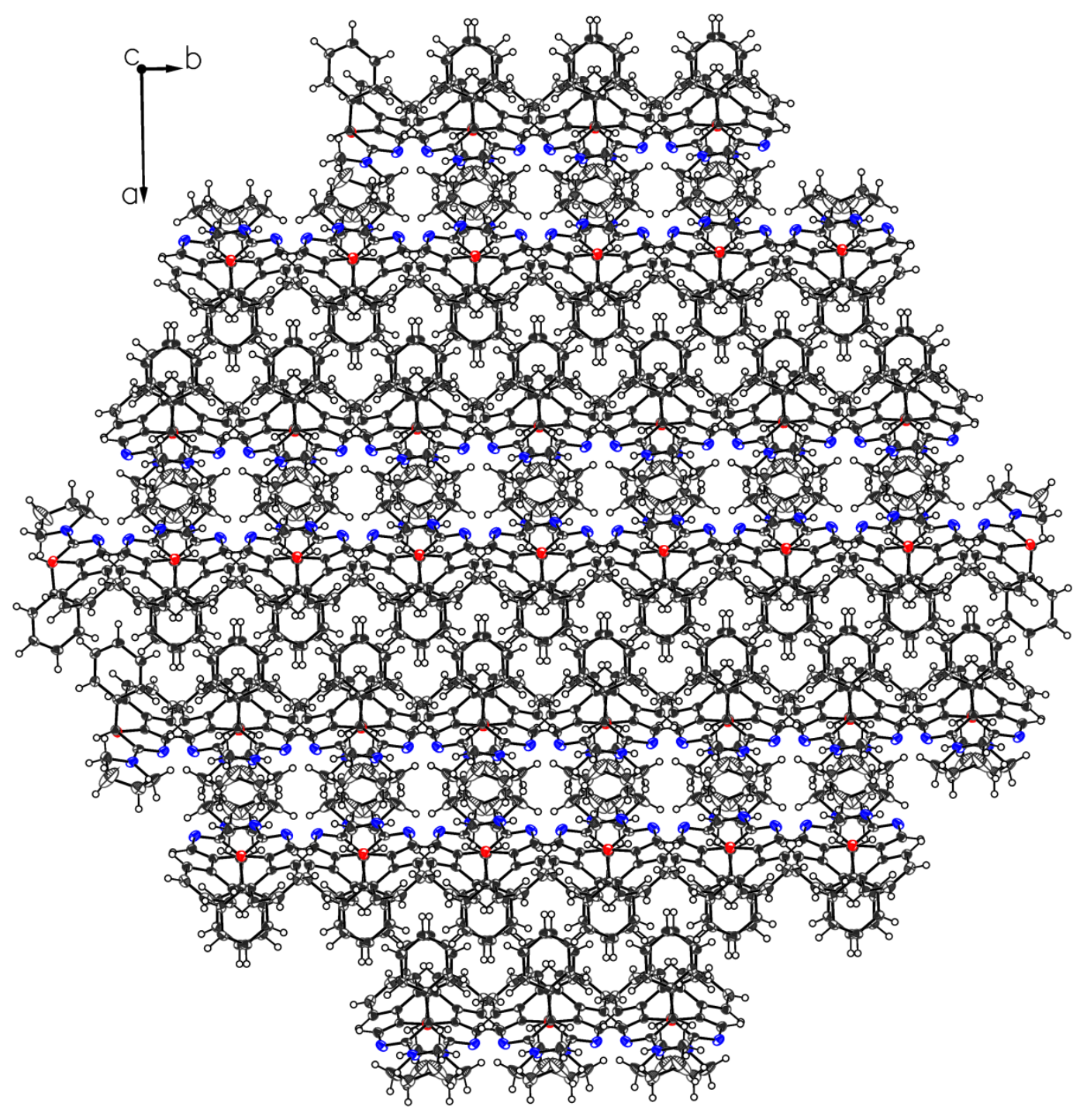

Figure S2 Packing view. 
Table S4 Sample and crystal data.

\begin{tabular}{|c|c|c|c|c|}
\hline Chemical formula & $\mathrm{C} 20 \mathrm{H} 20 \mathrm{~N} 2 \mathrm{O}$ & Crystal system & \multicolumn{2}{|c|}{ monoclinic } \\
\hline Formula weight $[\mathrm{g} / \mathrm{mol}]$ & 304.38 & Space group & \multicolumn{2}{|c|}{$C 2 / c$} \\
\hline Temperature [K] & 120 & $\mathbf{Z}$ & \multicolumn{2}{|c|}{8} \\
\hline Measurement method & $\backslash$ f and $\backslash$ w scans & Volume $\left[\AA^{3}\right]$ & \multicolumn{2}{|c|}{$3128.3(3)$} \\
\hline $\begin{array}{l}\text { Radiation (Wavelength } \\
[\AA \AA]]\end{array}$ & $\operatorname{MoK} \alpha(\lambda=0.71073)$ & $\begin{array}{l}\text { Unit cell } \\
\text { dimensions }[\AA \AA] \\
\text { and }\left[{ }^{\circ}\right] \\
\end{array}$ & $31.2597(15)$ & 90 \\
\hline Crystal size / $\left[\mathrm{mm}^{3}\right]$ & $0.581 \times 0.148 \times 0.046$ & & $5.7581(3)$ & $116.0874(16)$ \\
\hline Crystal habit & clear yellow plate & & $19.3514(9)$ & 90 \\
\hline $\begin{array}{l}\text { Density (calculated) / } \\
{\left[\mathrm{g} / \mathrm{cm}^{3}\right]}\end{array}$ & 1.293 & $\begin{array}{l}\text { Absorption } \\
\text { coefficient } /\left[\mathrm{mm}^{-1}\right]\end{array}$ & \multicolumn{2}{|c|}{0.08} \\
\hline Abs. correction Tmin & 0.3486 & $\begin{array}{l}\text { Abs. correction } \\
\text { Tmax }\end{array}$ & \multicolumn{2}{|c|}{0.746} \\
\hline Abs. correction type & multiscan & $\mathbf{F}(000)\left[\mathrm{e}^{-}\right]$ & \multicolumn{2}{|c|}{1296} \\
\hline
\end{tabular}

Table S5 Data collection and structure refinement.

\begin{tabular}{|c|c|c|c|c|}
\hline Index ranges & $\begin{array}{c}-37 \leq \mathrm{h} \leq 37,-6 \leq \mathrm{k} \leq 6 \\
-23 \leq 1 \leq 21\end{array}$ & $\begin{array}{l}\text { Theta range for } \\
\text { data collection }\left[{ }^{\circ}\right]\end{array}$ & \multicolumn{2}{|r|}{4.688 to 50.678} \\
\hline Reflections number & 26343 & $\begin{array}{l}\text { Data / restraints / } \\
\text { parameters }\end{array}$ & \multicolumn{2}{|r|}{$2846 / 6 / 212$} \\
\hline Refinement method & Least squares & \multirow{2}{*}{ Final $R$ indices } & all data & $\mathrm{R} 1=0.0682, \mathrm{wR} 2=0.1648$ \\
\hline Function minimized & $\Sigma \mathrm{w}\left(\mathrm{F}_{\mathrm{o}}^{2}-\mathrm{F}_{\mathrm{c}}^{2}\right)^{2}$ & & $\mathrm{I}>2 \sigma(\mathrm{I})$ & $\mathrm{R} 1=0.0625, \mathrm{wR} 2=0.1588$ \\
\hline Goodness-of-fit on $\mathbf{F}^{2}$ & 1.054 & \multirow[b]{2}{*}{ Weighting scheme } & \multicolumn{2}{|c|}{$\mathrm{w}=1 /[\sigma 2(\mathrm{Fo} 2)+(0.0747 \mathrm{P}) 2+7.3558 \mathrm{P}]$} \\
\hline $\begin{array}{l}\text { Largest diff. peak and } \\
\text { hole }\left[\mathrm{e} \AA^{-3}\right]\end{array}$ & $0.65 /-0.57$ & & \multicolumn{2}{|c|}{ where $\mathrm{P}=\left(\mathrm{F}_{\mathrm{o}}^{2}+2 \mathrm{~F}_{\mathrm{c}}^{2}\right) / 3$} \\
\hline
\end{tabular}



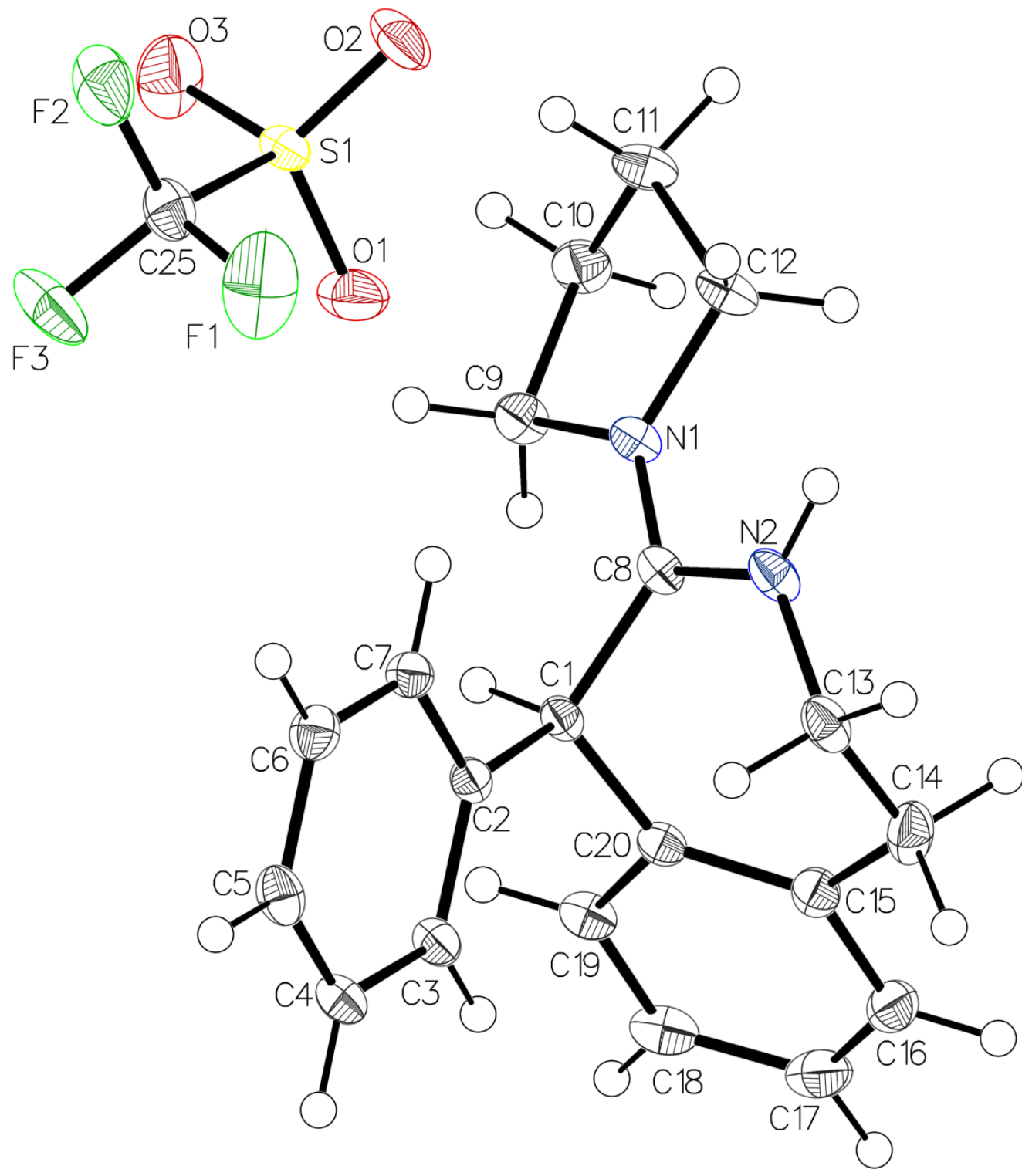

Figure S3 Crystal structure, drawn with 50\% displacement ellipsoid. The bond precision for C-C single bonds is $0.0043 \AA$. 


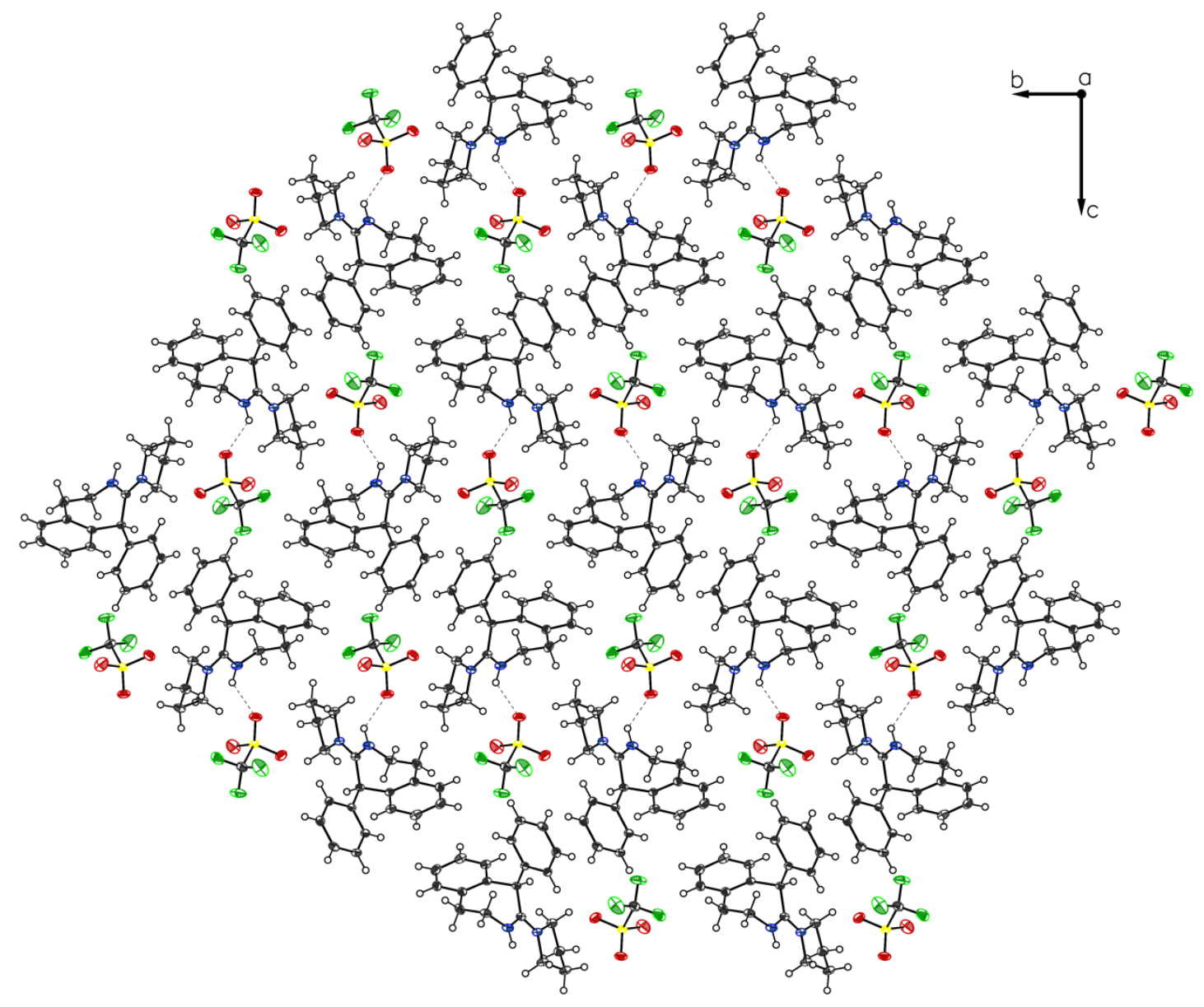

Figure S4 In the packing view one hydrogen bond on trifluoromethanesulfonate was detected with a bond length $2.083 \AA$.

Table S6 Sample and crystal data.

\begin{tabular}{|c|c|c|c|c|}
\hline Chemical formula & $\mathrm{C} 21 \mathrm{H} 23 \mathrm{~F} 3 \mathrm{~N} 2 \mathrm{O} 3 \mathrm{~S}$ & Crystal system & \multicolumn{2}{|c|}{ orthorhombic } \\
\hline Formula weight $[\mathrm{g} / \mathrm{mol}]$ & 440.47 & Space group & \multicolumn{2}{|r|}{$P 212121$} \\
\hline Temperature [K] & 100 & $\mathbf{Z}$ & \multicolumn{2}{|r|}{4} \\
\hline Measurement method & $\backslash$ f and $\backslash w$ scans & Volume $\left[\AA^{3}\right]$ & \multicolumn{2}{|c|}{$2034.84(8)$} \\
\hline $\begin{array}{l}\text { Radiation (Wavelength } \\
[\AA \AA]]\end{array}$ & $\operatorname{MoK} \alpha(\lambda=0.71073)$ & $\begin{array}{l}\text { Unit cell } \\
\text { dimensions }[\AA]] \\
\text { and }\left[{ }^{\circ}\right]\end{array}$ & $6.48180(10)$ & 90 \\
\hline Crystal size / $\left[\mathrm{mm}^{3}\right]$ & $0.579 \times 0.244 \times 0.144$ & & $12.5595(3)$ & 90 \\
\hline Crystal habit & clear colourless block & & $24.9955(6)$ & 90 \\
\hline $\begin{array}{l}\text { Density (calculated) / } \\
{\left[\mathrm{g} / \mathrm{cm}^{3}\right]}\end{array}$ & 1.438 & $\begin{array}{l}\text { Absorption } \\
\text { coefficient } /\left[\mathrm{mm}^{-1}\right]\end{array}$ & \multicolumn{2}{|r|}{0.212} \\
\hline Abs. correction Tmin & 0.6553 & $\begin{array}{l}\text { Abs. correction } \\
\text { Tmax }\end{array}$ & \multicolumn{2}{|r|}{0.7467} \\
\hline Abs. correction type & multiscan & $\mathbf{F}(000)\left[\mathrm{e}^{-}\right]$ & \multicolumn{2}{|r|}{920} \\
\hline
\end{tabular}


Table S7 Data collection and structure refinement.

\begin{tabular}{|c|c|c|c|c|}
\hline Index ranges & $\begin{array}{c}-9 \leq \mathrm{h} \leq 8,-17 \leq \mathrm{k} \leq 19 \\
-38 \leq 1 \leq 39\end{array}$ & $\begin{array}{l}\text { Theta range for } \\
\text { data collection }\left[{ }^{\circ}\right]\end{array}$ & \multicolumn{2}{|c|}{4.598 to 68.018} \\
\hline Reflections number & 21905 & $\begin{array}{l}\text { Data / restraints / } \\
\text { parameters }\end{array}$ & \multicolumn{2}{|r|}{$7198 / 0 / 271$} \\
\hline Refinement method & Least squares & \multirow{2}{*}{ Final $\mathrm{R}$ indices } & all data & $\mathrm{R} 1=0.0615, \mathrm{wR} 2=0.1365$ \\
\hline Function minimized & $\Sigma \mathrm{w}\left(\mathrm{F}_{\mathrm{o}}^{2}-\mathrm{F}_{\mathrm{c}}^{2}\right)^{2}$ & & $\mathrm{I}>2 \sigma(\mathrm{I})$ & $\mathrm{R} 1=0.0433, \mathrm{wR} 2=0.0970$ \\
\hline Goodness-of-fit on $\mathbf{F}^{2}$ & 1.185 & \multirow[b]{2}{*}{ Weighting scheme } & \multicolumn{2}{|c|}{$\mathrm{w}=1 /[\sigma 2(\mathrm{Fo} 2)+2.4717 \mathrm{P}]$} \\
\hline $\begin{array}{l}\text { Largest diff. peak and } \\
\text { hole }\left[e \AA^{-3}\right]\end{array}$ & $0.59 /-0.72$ & & \multicolumn{2}{|c|}{ where $\mathrm{P}=\left(\mathrm{F}_{\mathrm{o}}^{2}+2 \mathrm{~F}_{\mathrm{c}}^{2}\right) / 3$} \\
\hline
\end{tabular}


6. NMR

${ }^{1} \mathbf{H}$ NMR (400 MHz, $\mathrm{CDCl}_{3}$ )

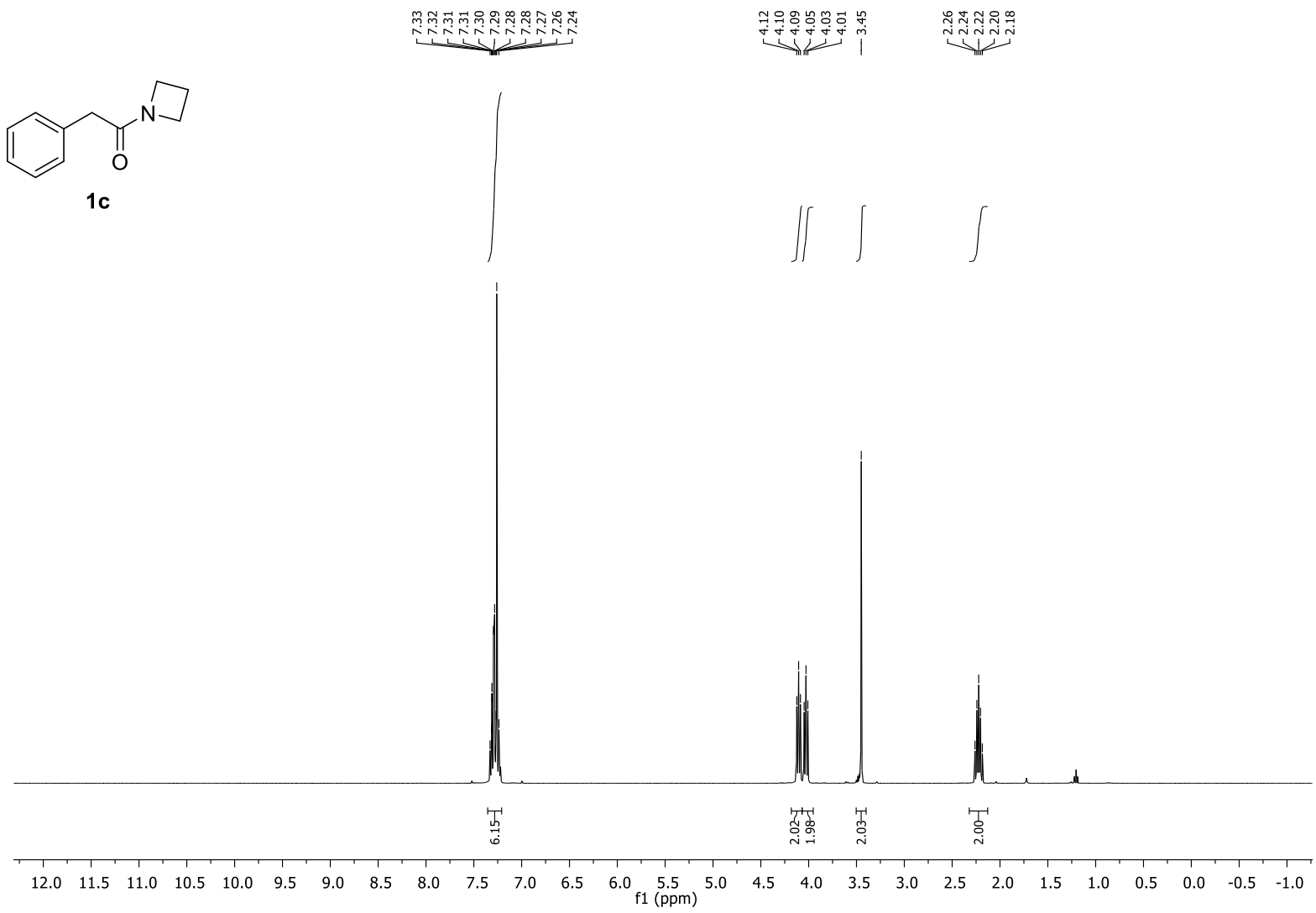

${ }^{13} \mathrm{C}$ NMR (151 MHz, $\left.\mathrm{CDCl}_{3}\right)$

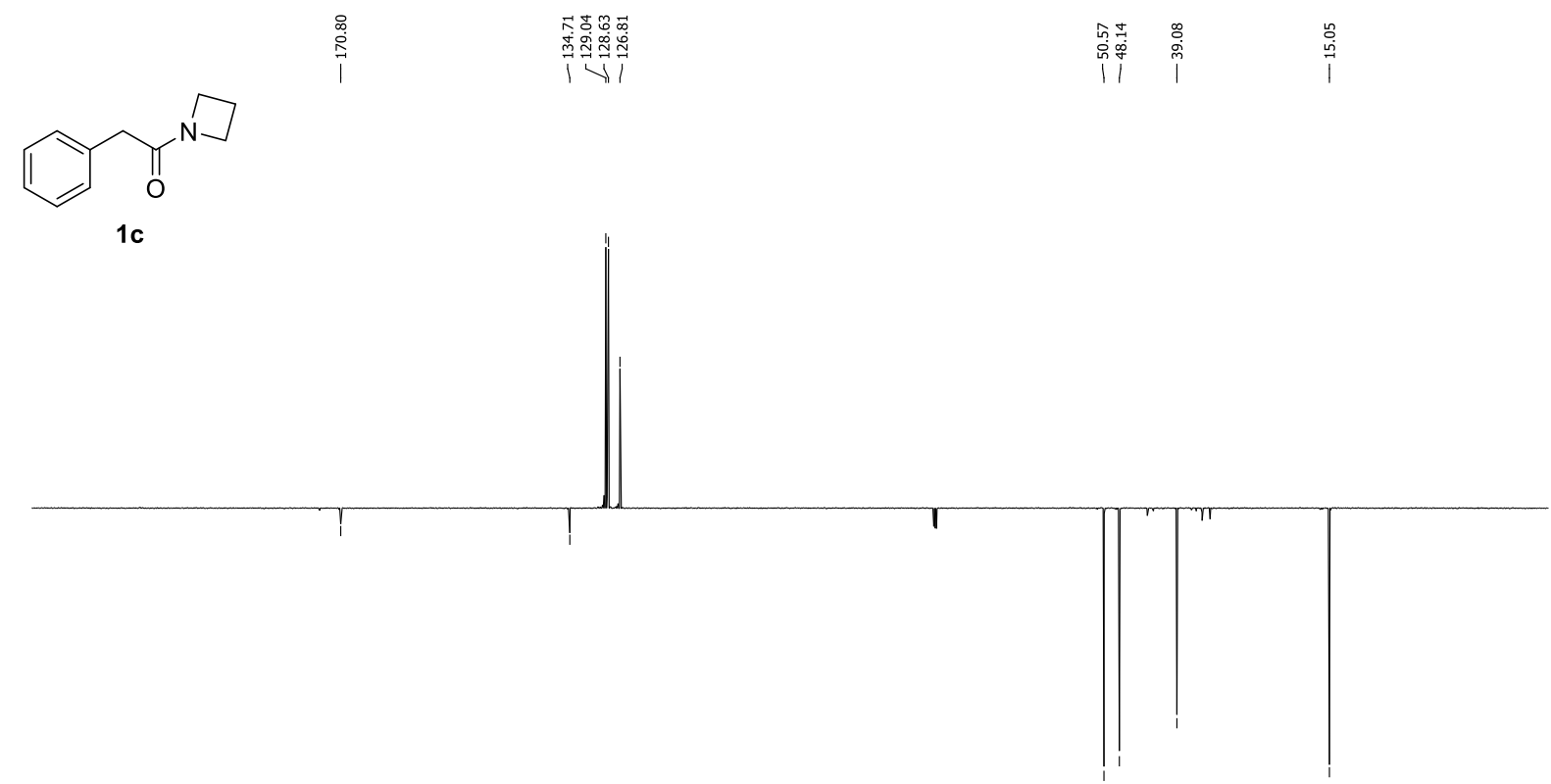

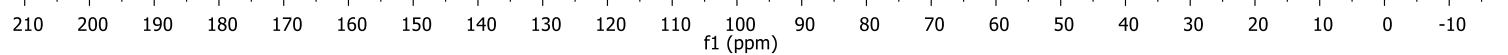


${ }^{1} \mathrm{H}$ NMR $\left(400 \mathrm{MHz}, \mathrm{CDCl}_{3}\right)$

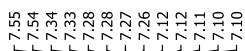

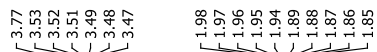<smiles>O=C(Cc1ccccc1Br)N1CCCC1</smiles>

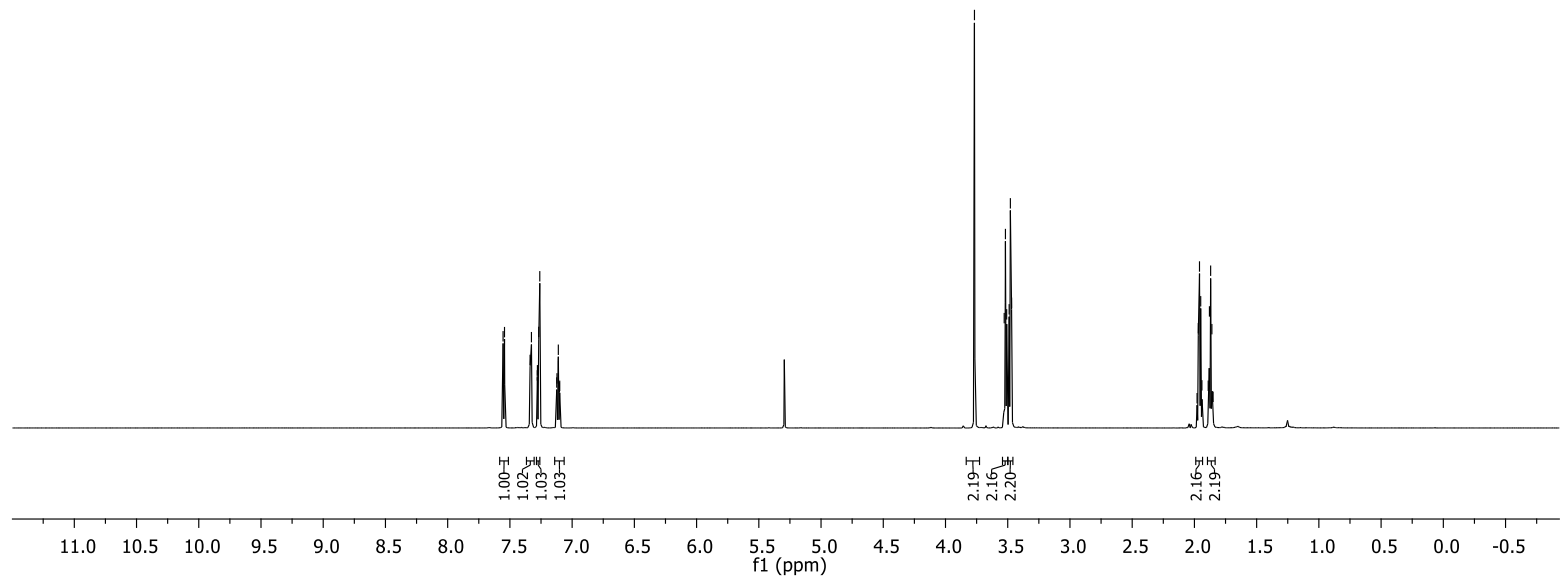

${ }^{13} \mathrm{C}$ NMR $\left(151 \mathrm{MHz}, \mathrm{CDCl}_{3}\right)$

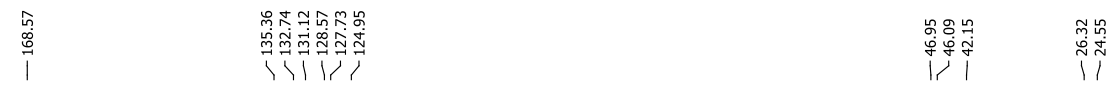<smiles>O=C(Cc1ccccc1Br)N1CCCC1</smiles>

1d

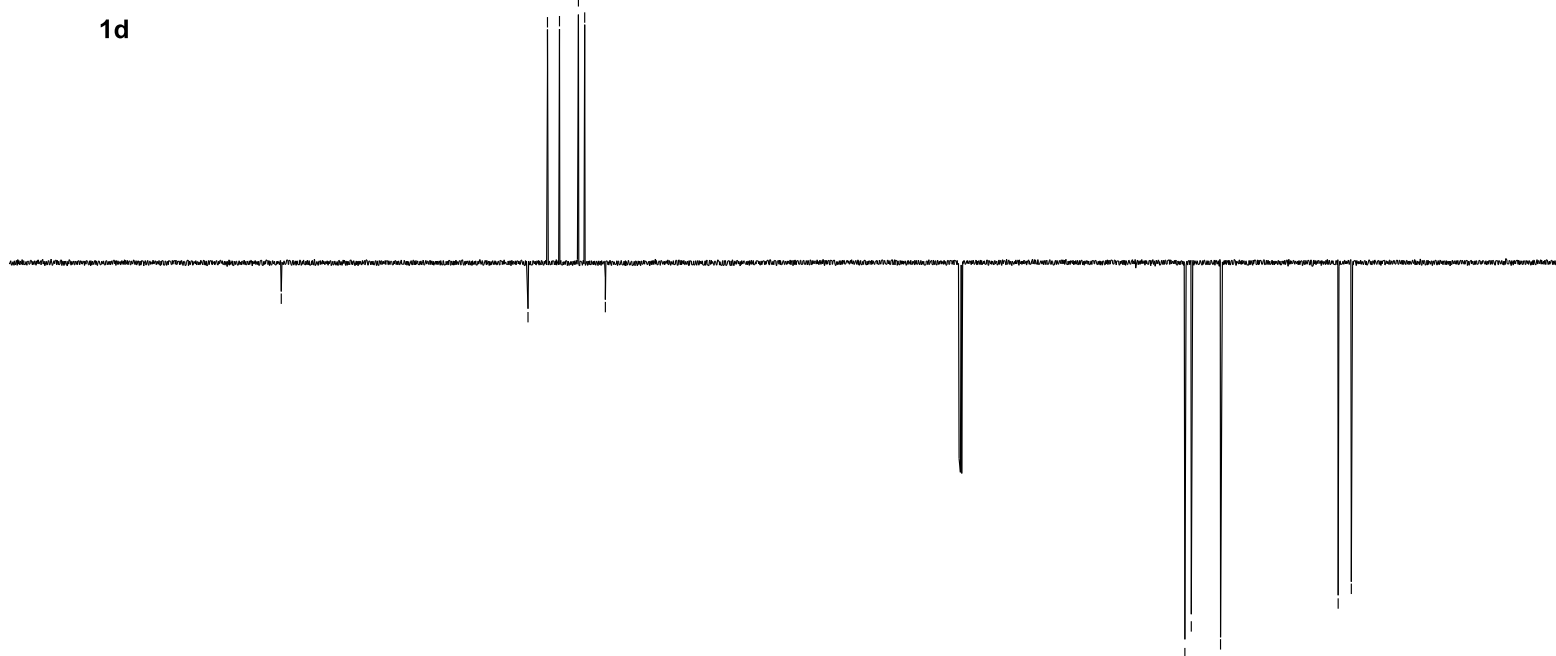

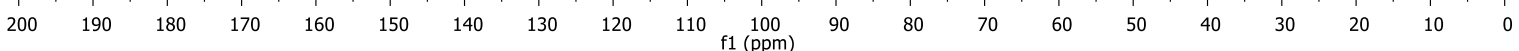


${ }^{1} \mathbf{H}$ NMR $\left(600 \mathrm{MHz}, \mathrm{CDCl}_{3}\right)$

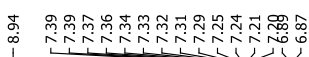

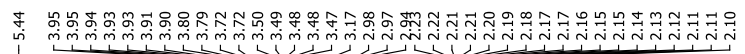

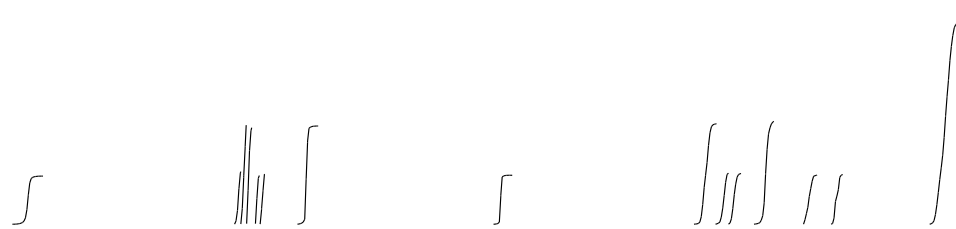<smiles>[O-][NH+]1CCc2ccccc2C(c2ccccc2)C1N1CCCC1</smiles>

${ }^{13} \mathrm{C}$ NMR (151 MHz, $\left.\mathrm{CDCl}_{3}\right)$

$\begin{array}{ll}0 & \\ 0 & 0\end{array}$

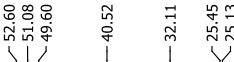<smiles>[O-][NH+]1CCc2ccccc2C(c2ccccc2)C1N1CCCC1</smiles>

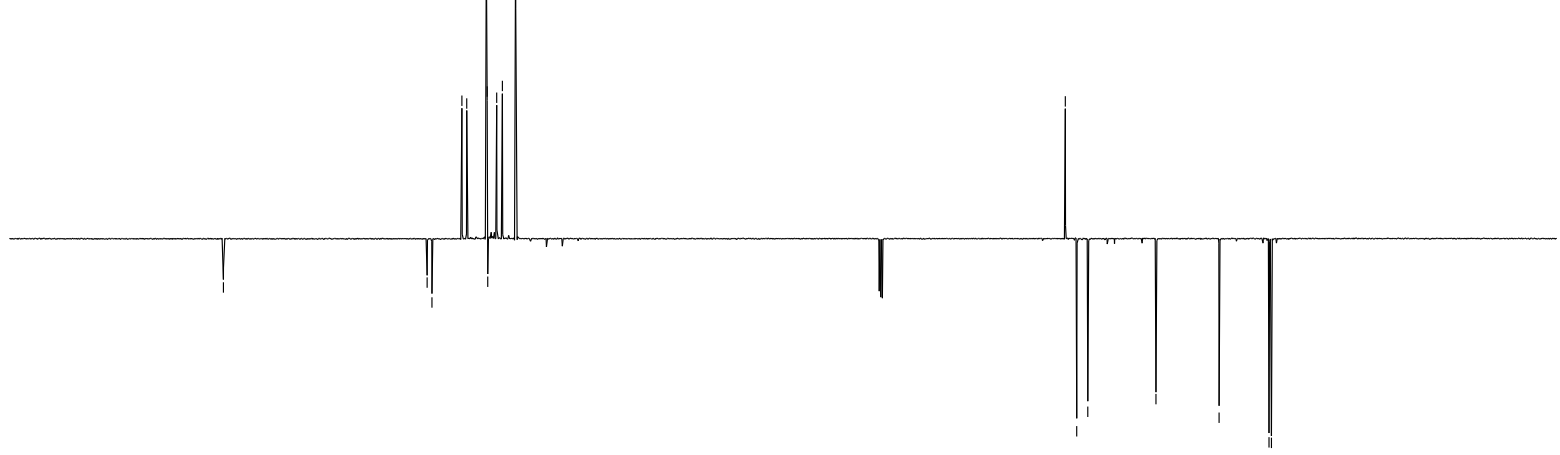

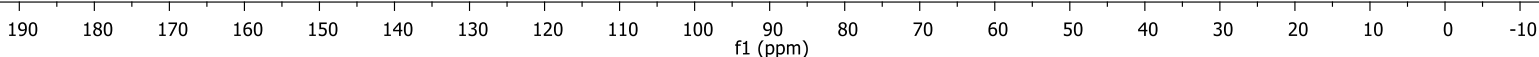


${ }^{19}$ F NMR (565 MHz, $\mathrm{CDCl}_{3}$ )
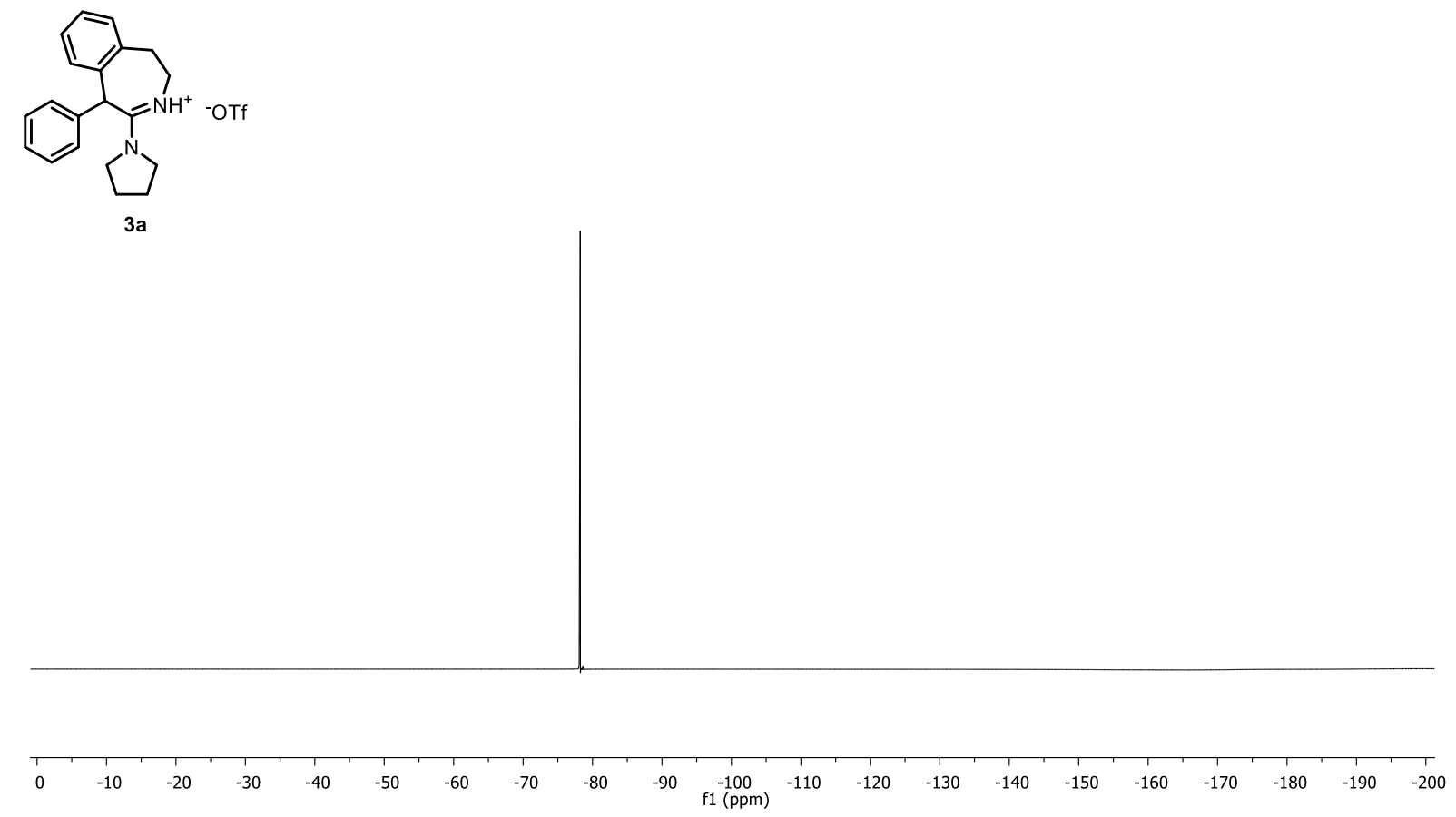

${ }^{1} \mathbf{H} \mathbf{N M R}\left(600 \mathrm{MHz}, \mathrm{CDCl}_{3}\right)$

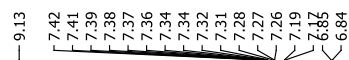

|

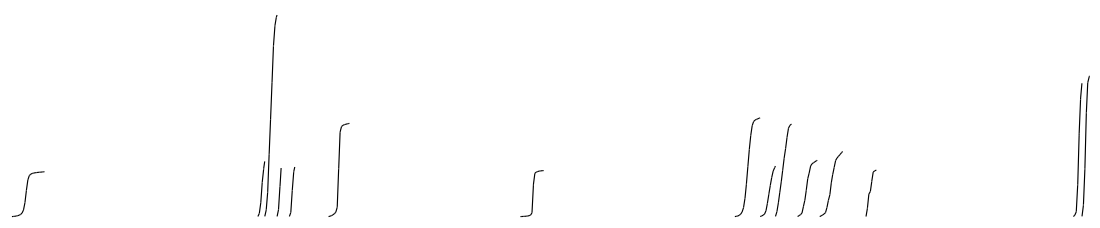<smiles>CCN(CC)C1=[NH+]CCc2ccccc2C1c1ccccc1</smiles>

$3 b$

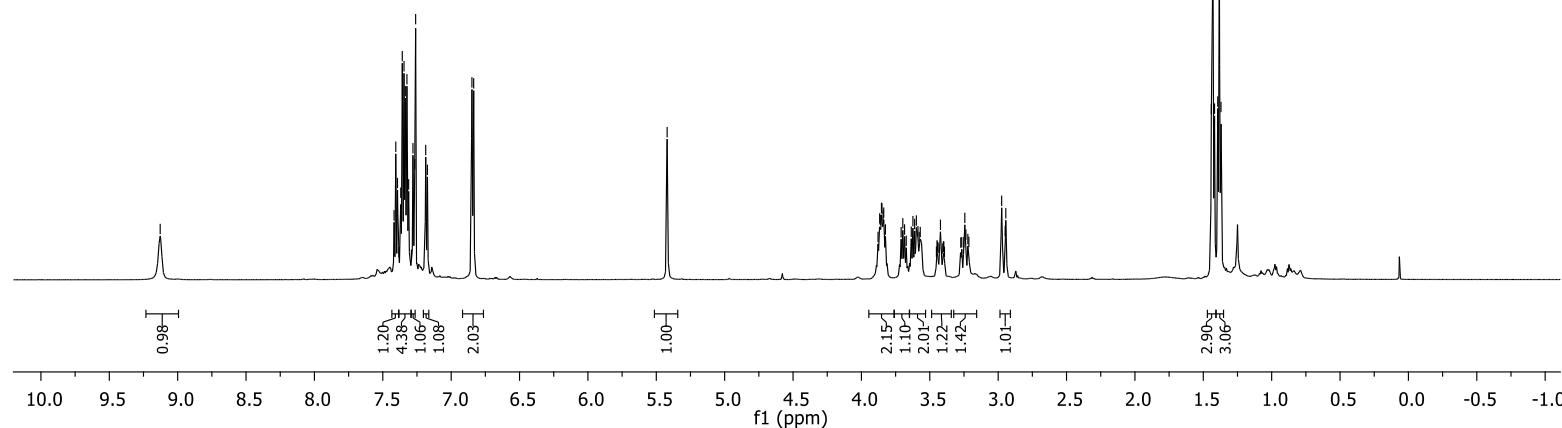


${ }^{13} \mathrm{C}$ NMR $\left(151 \mathrm{MHz}, \mathrm{CDCl}_{3}\right)$

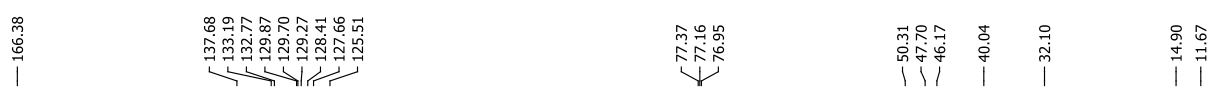<smiles>CCN(CC)C1=[NH+]CCc2ccccc2C1c1ccccc1</smiles>

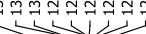

|广।

I

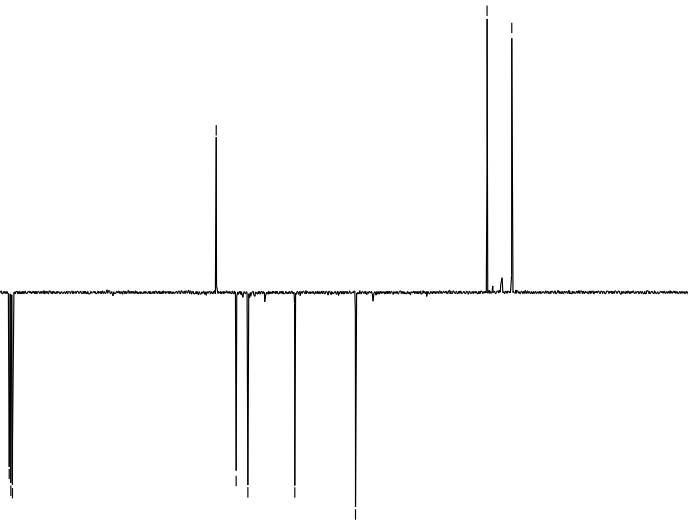

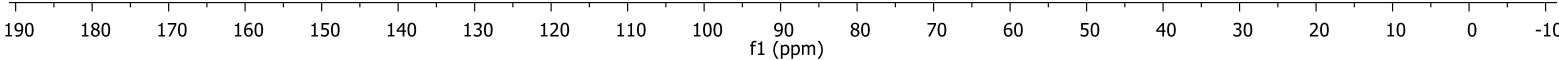

${ }^{19} \mathrm{~F}$ NMR $\left(565 \mathrm{MHz}, \mathrm{CDCl}_{3}\right)$

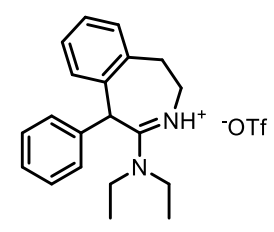

3b
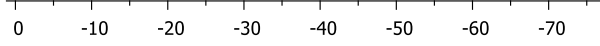
${ }^{1} \mathrm{H}$ NMR (600 MHz, $\mathrm{CDCl}_{3}$ )
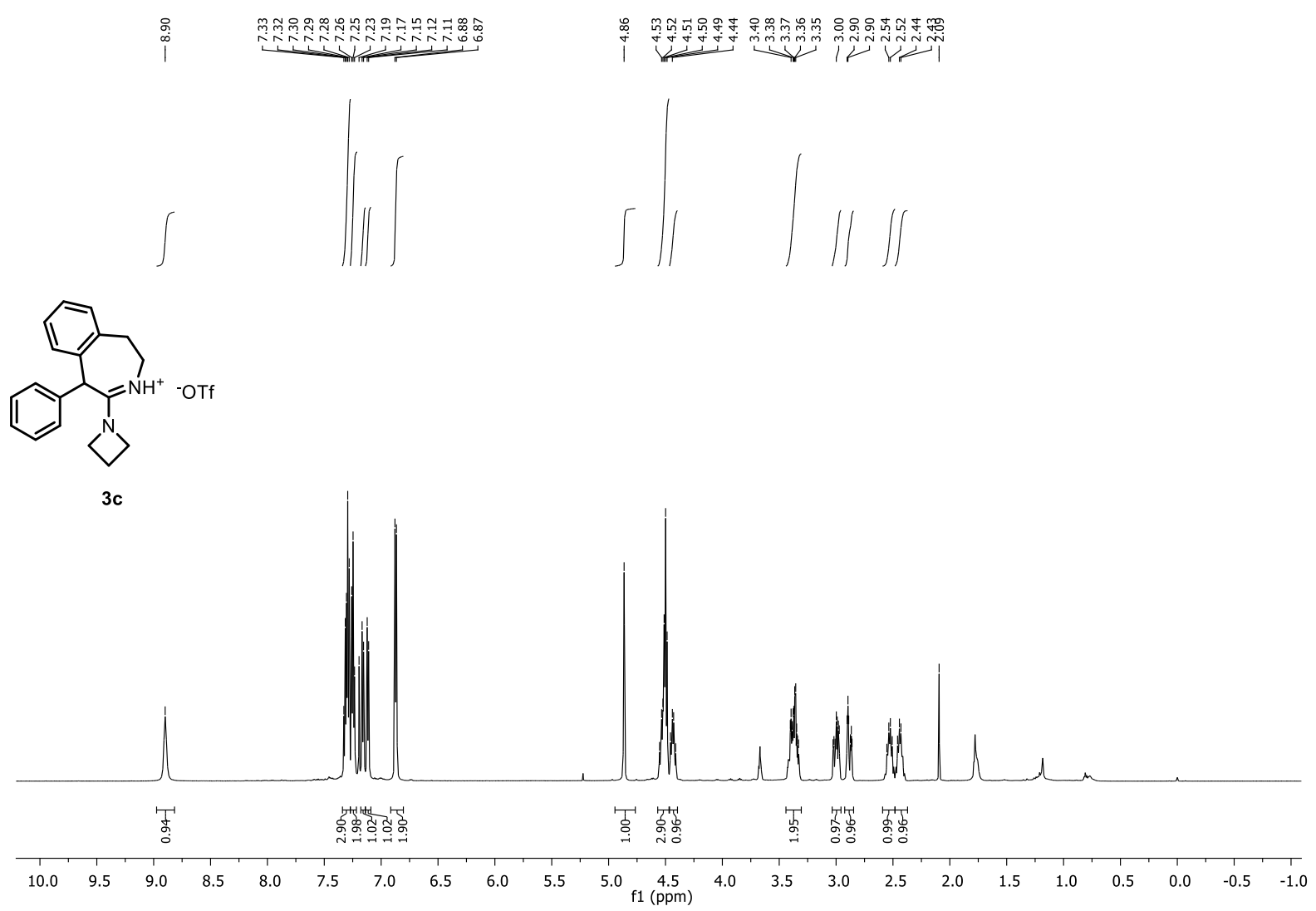

${ }^{13}$ C NMR (151 MHz, $\left.\mathrm{CDCl}_{3}\right)$

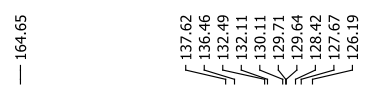

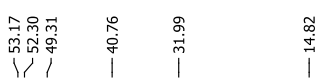<smiles>[O-][NH+]1CCc2ccccc2C(c2ccccc2)C1N1CCC1</smiles>

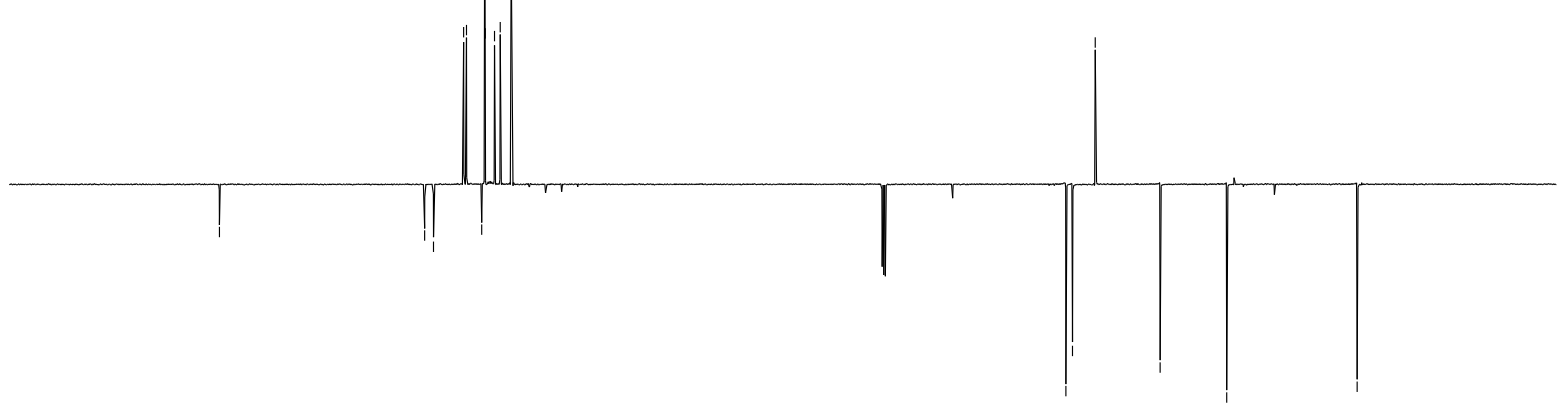

190

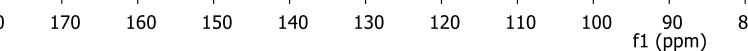


${ }^{19} \mathrm{~F}$ NMR (565 MHz, $\mathrm{CDCl}_{3}$ )<smiles>[O-][NH+]1CCc2ccccc2C(c2ccccc2)C1N1CCC1</smiles>

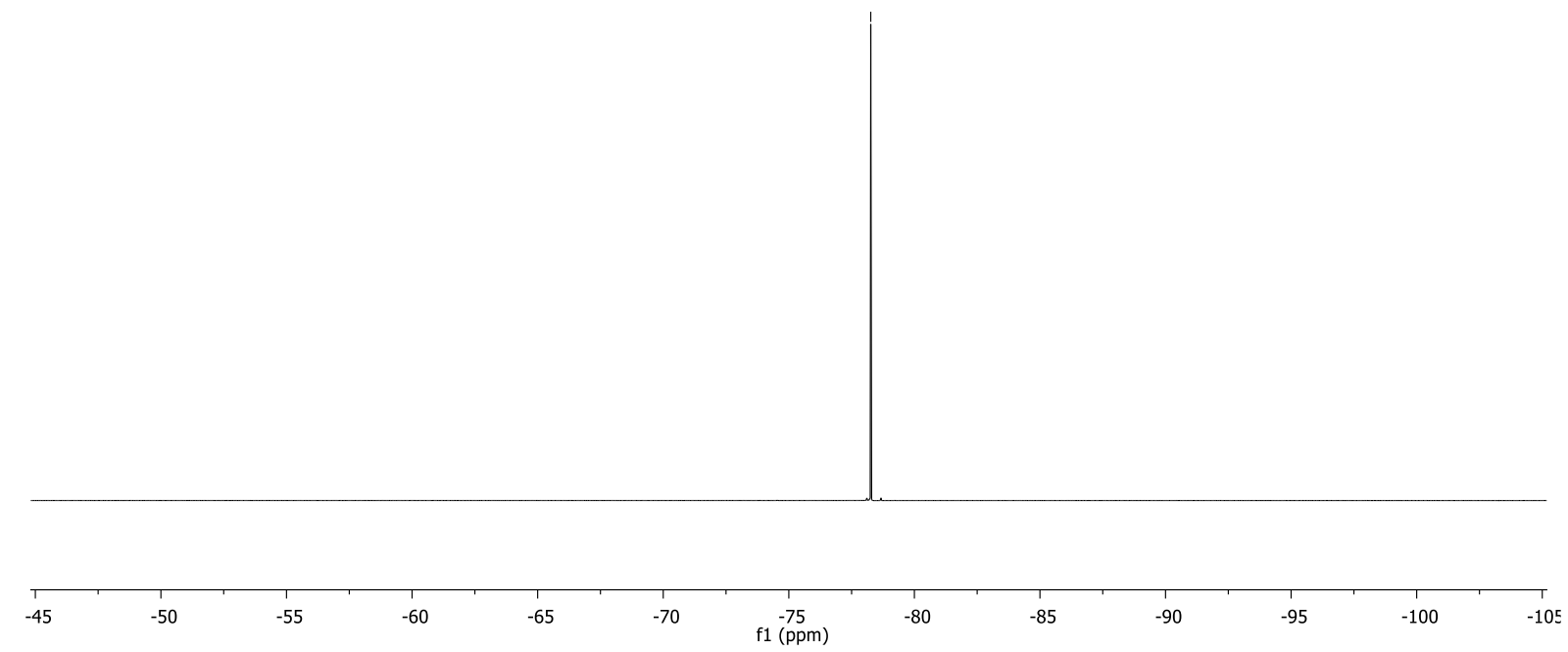

${ }^{1} \mathbf{H} \mathbf{N M R}\left(600 \mathrm{MHz}, \mathrm{CDCl}_{3}\right)$
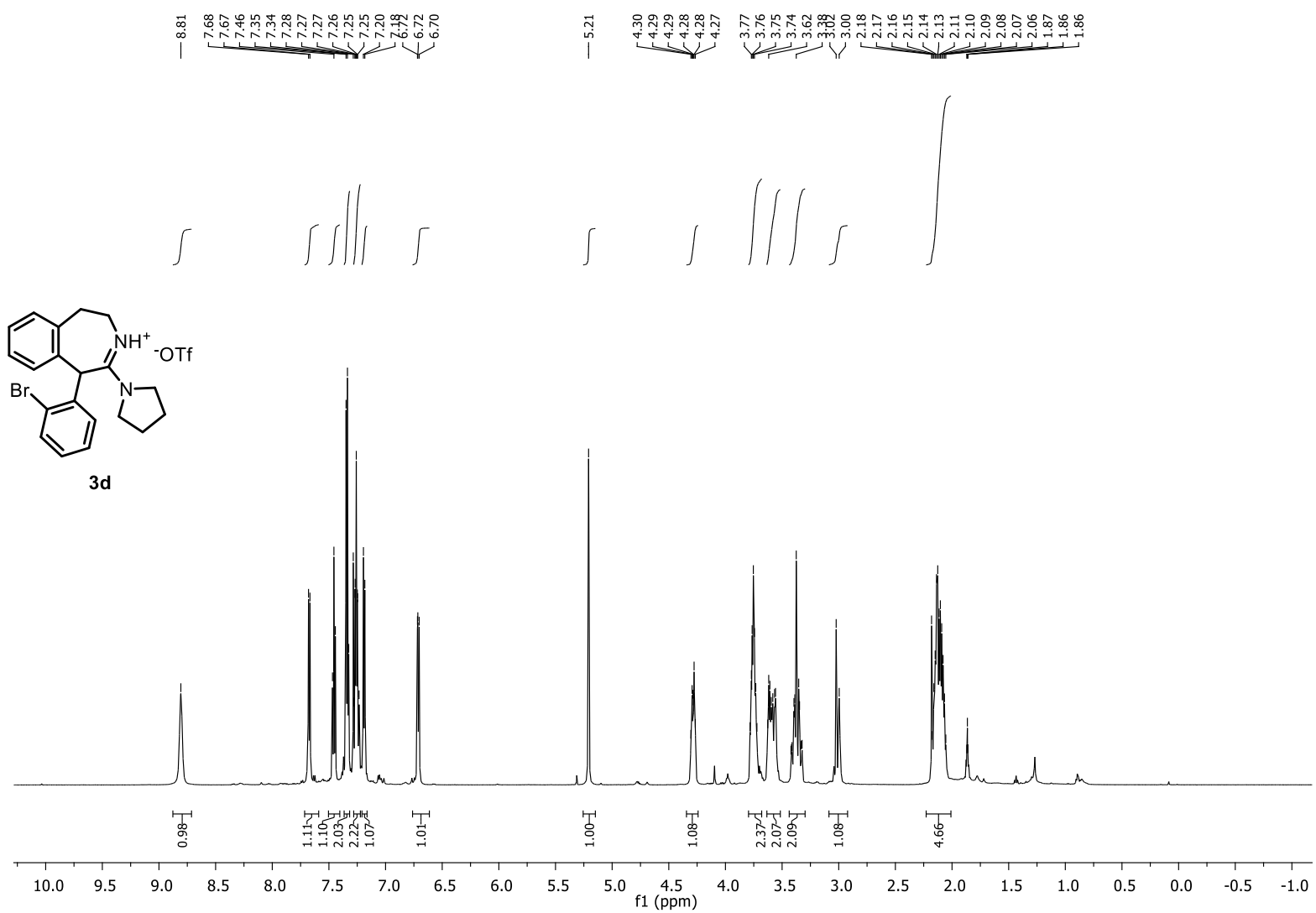
${ }^{13}$ C NMR (151 MHz, $\left.\mathrm{CDCl}_{3}\right)$

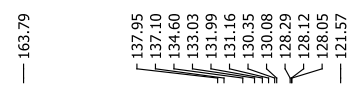

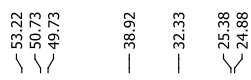

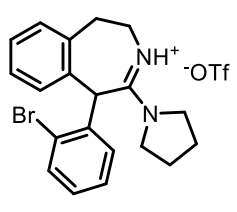

3d
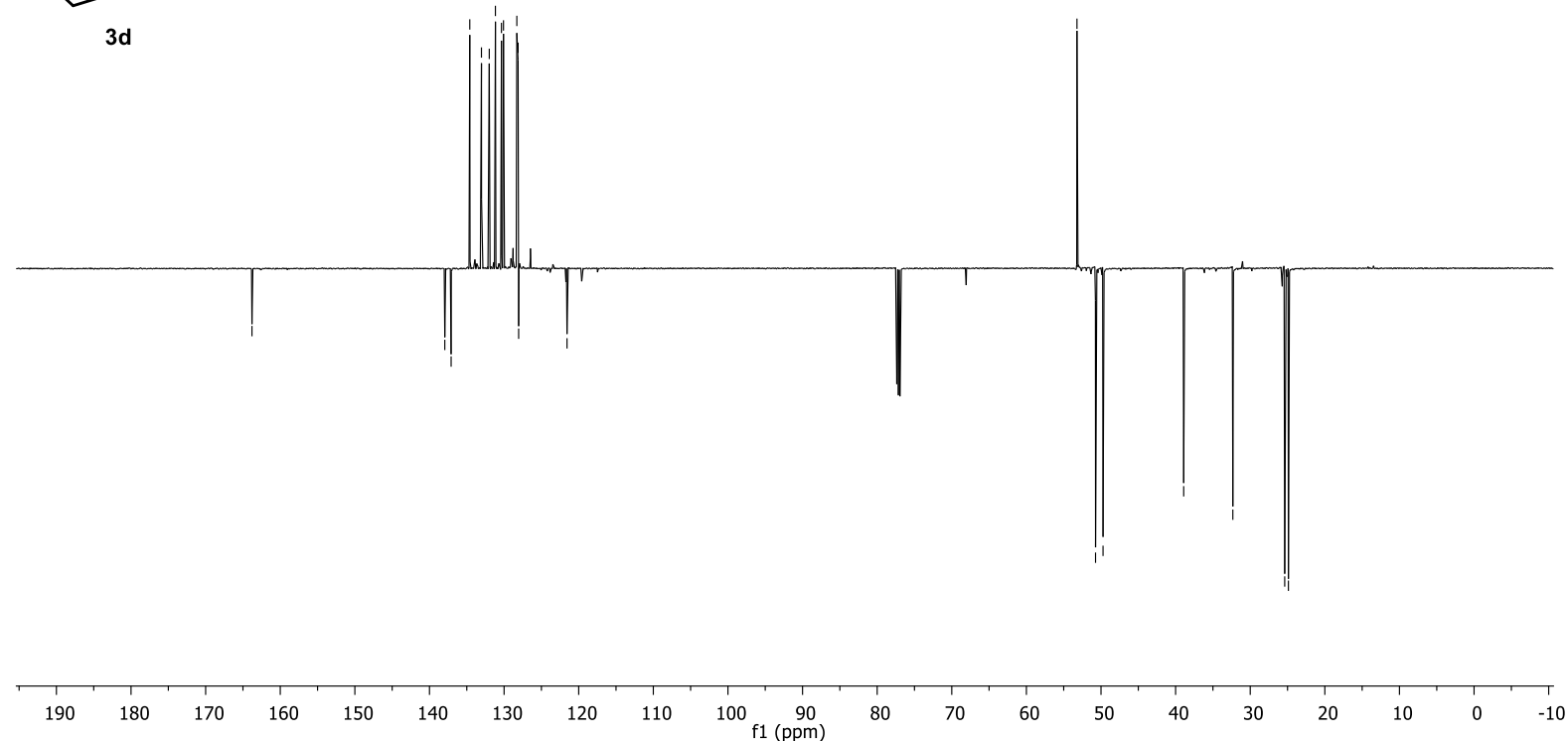

${ }^{19}$ F NMR (565 MHz, $\left.\mathrm{CDCl}_{3}\right)$

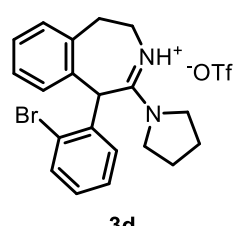

3d

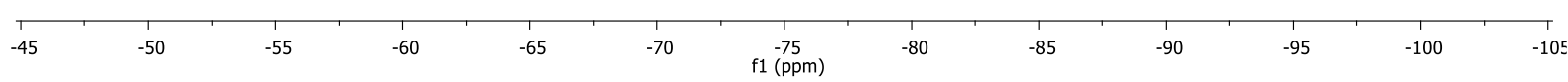


${ }^{1} \mathrm{H}$ NMR (400 MHz, $\left.\mathrm{CDCl}_{3}\right)$

|
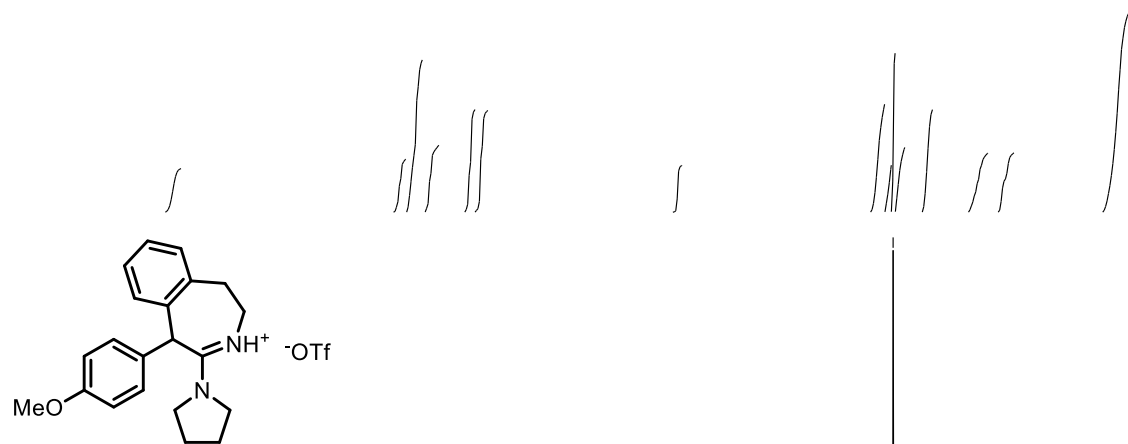

$3 e$

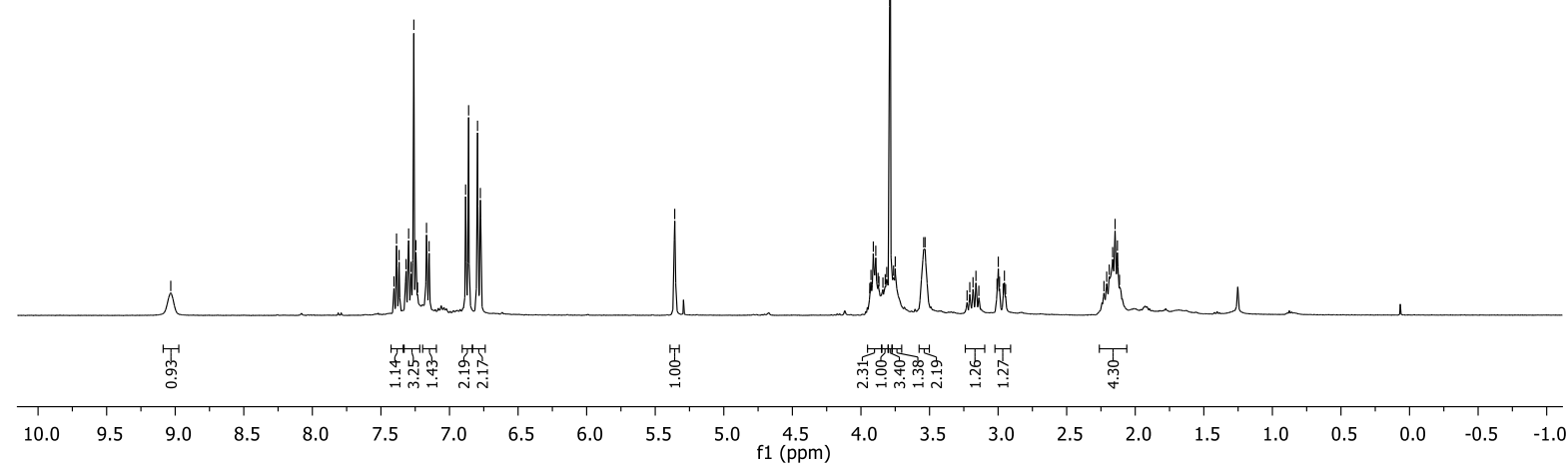

${ }^{13} \mathrm{C}$ NMR (151 MHz, $\left.\mathrm{CDCl}_{3}\right)$

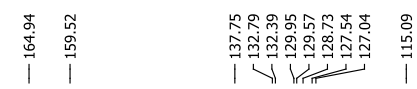

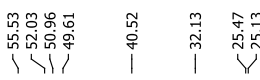
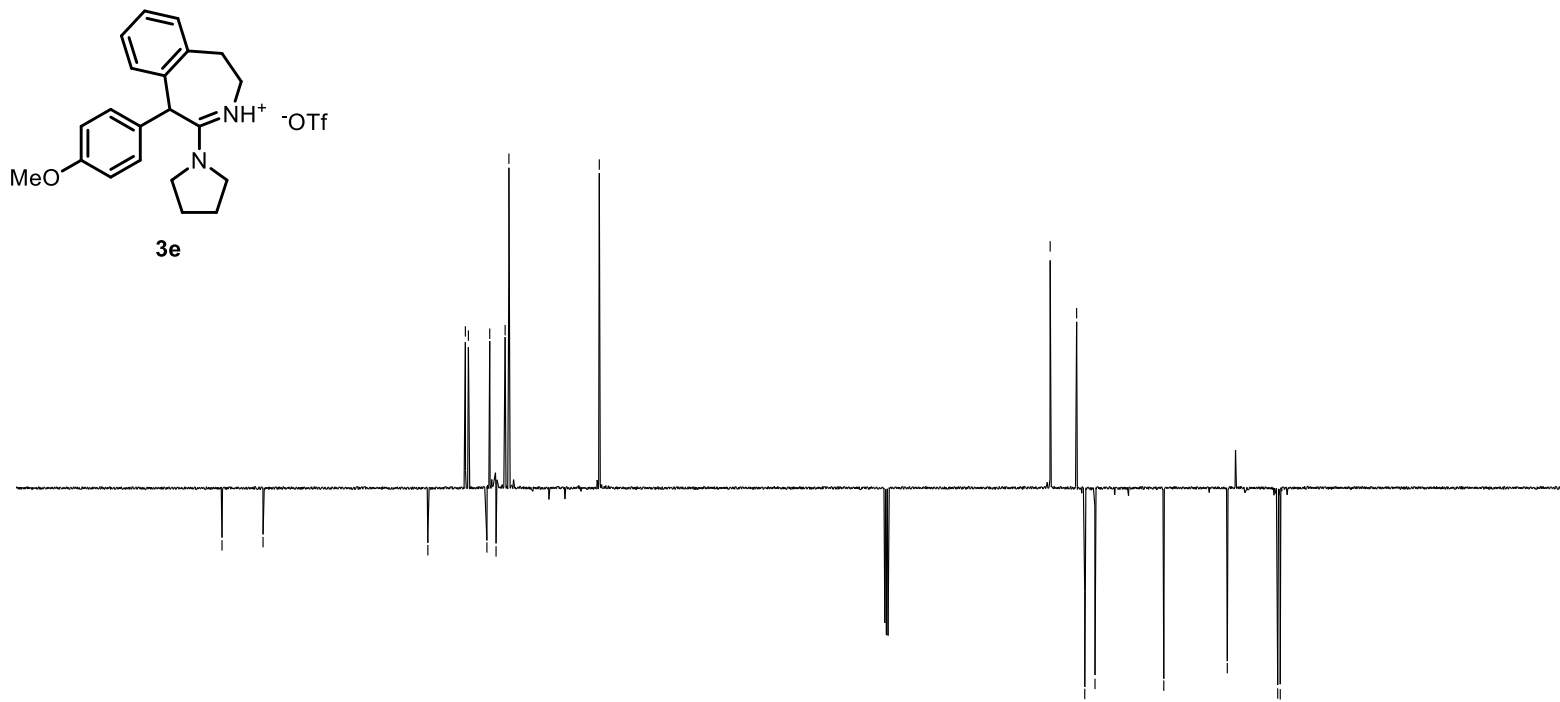

190

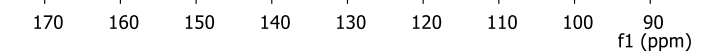


${ }^{19} \mathrm{~F}$ NMR $\left(565 \mathrm{MHz}, \mathrm{CDCl}_{3}\right)$

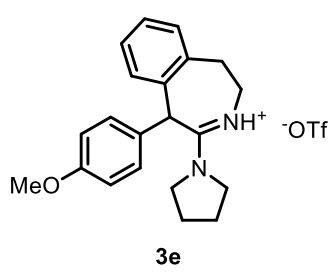

10

$\begin{array}{ccccccccc}-10 & -20 & -30 & -40 & -50 & -60 & -70 & -80 & -90 \quad \begin{array}{c}-100 \\ \mathrm{f} 1(\mathrm{ppm})\end{array}\end{array}$

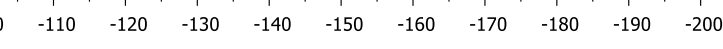

${ }^{1} \mathbf{H}$ NMR (600 $\left.\mathrm{MHz}, \mathrm{CDCl}_{3}\right)$

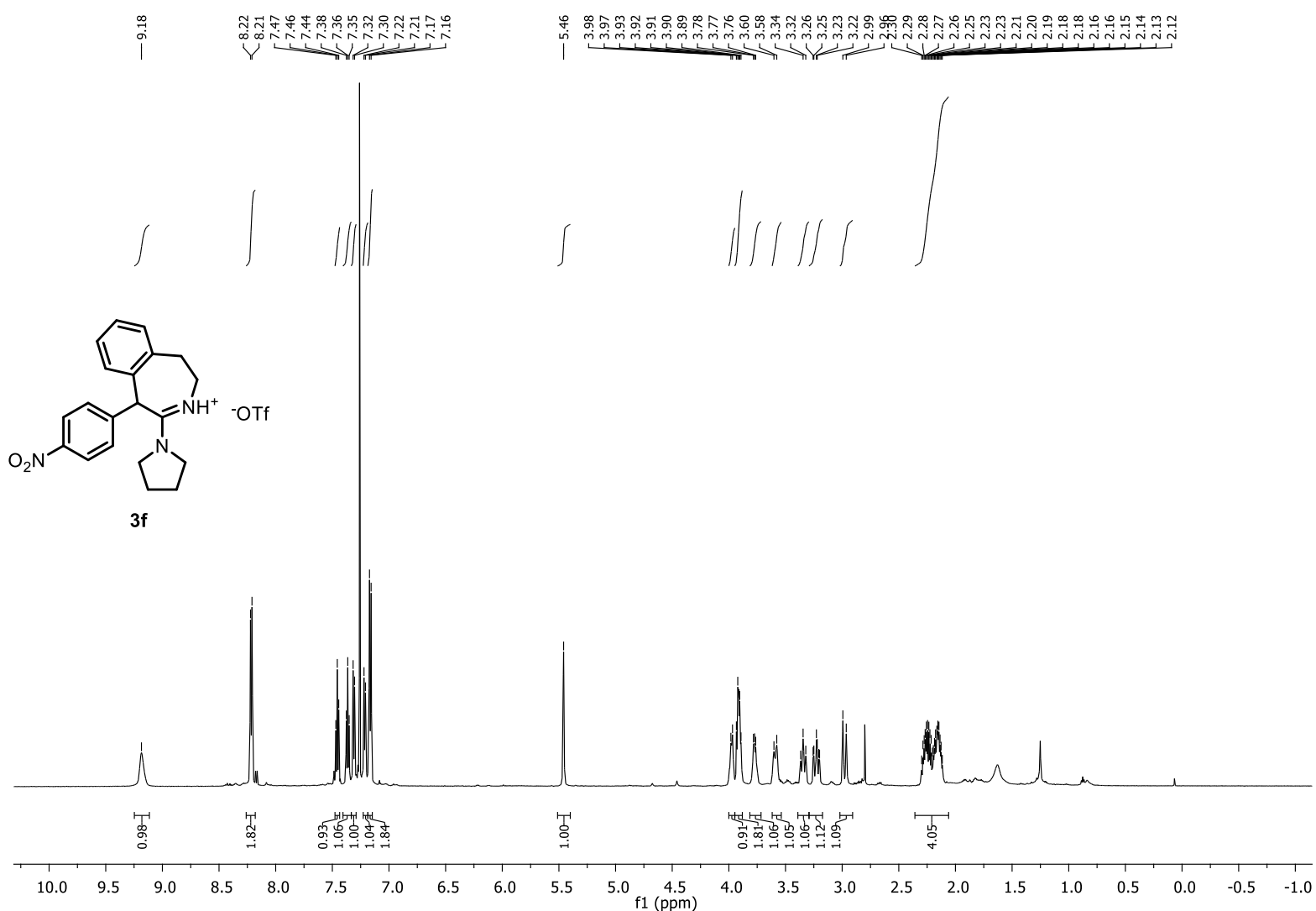


${ }^{13} \mathrm{C}$ NMR $\left(151 \mathrm{MHz}, \mathrm{CDCl}_{3}\right)$

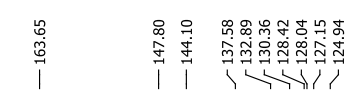

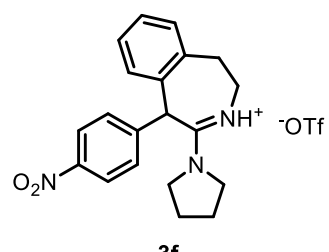

$3 f$

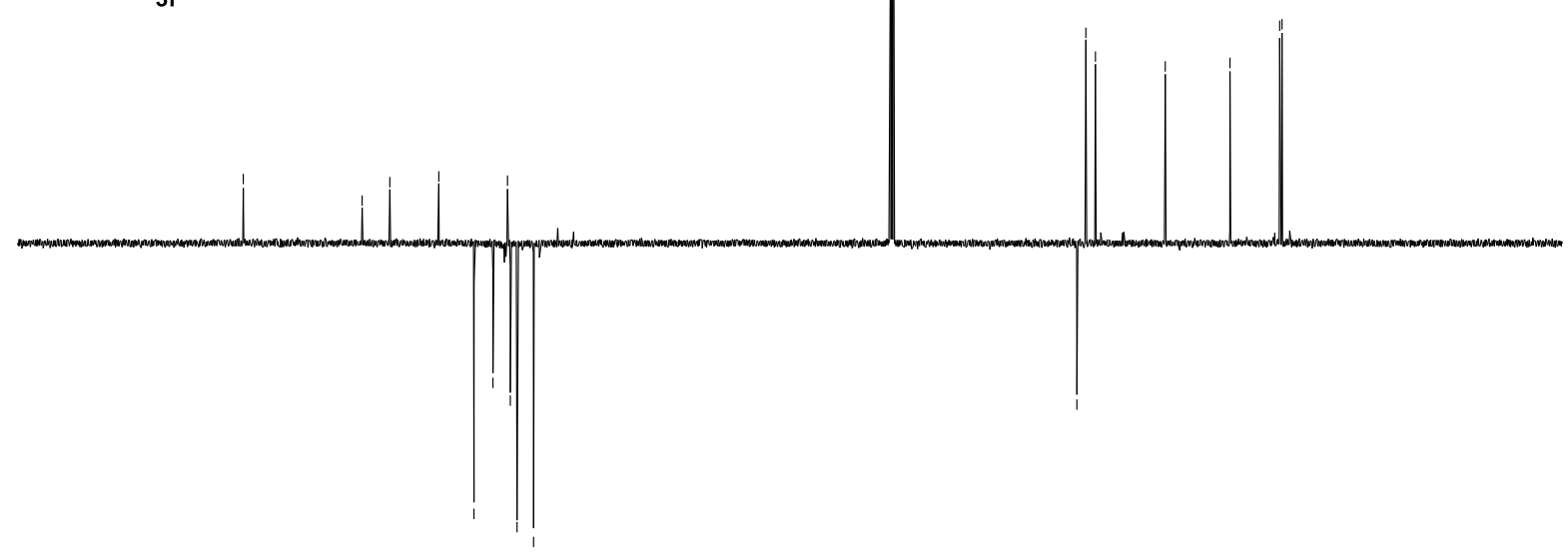

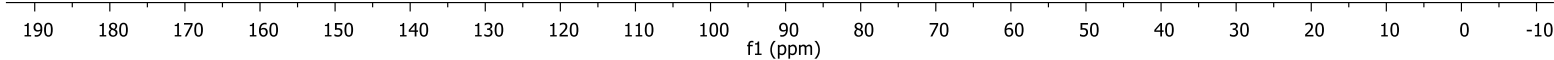

${ }^{19} \mathrm{~F}$ NMR $\left(565 \mathrm{MHz}, \mathrm{CDCl}_{3}\right)$

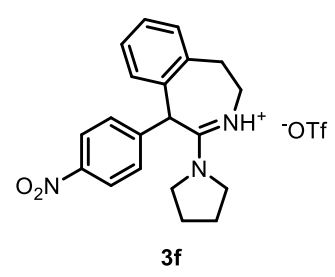


${ }^{1} \mathbf{H}$ NMR $\left(600 \mathrm{MHz}, \mathrm{CDCl}_{3}\right)$

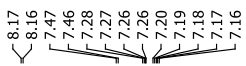

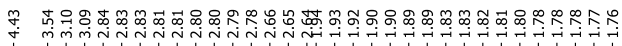<smiles>O=C(C(NCCc1ccccc1)c1ccc([N+](=O)[O-])cc1)N1CCCC1</smiles><smiles>C1=CCCC1</smiles>

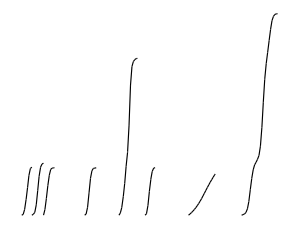

s4

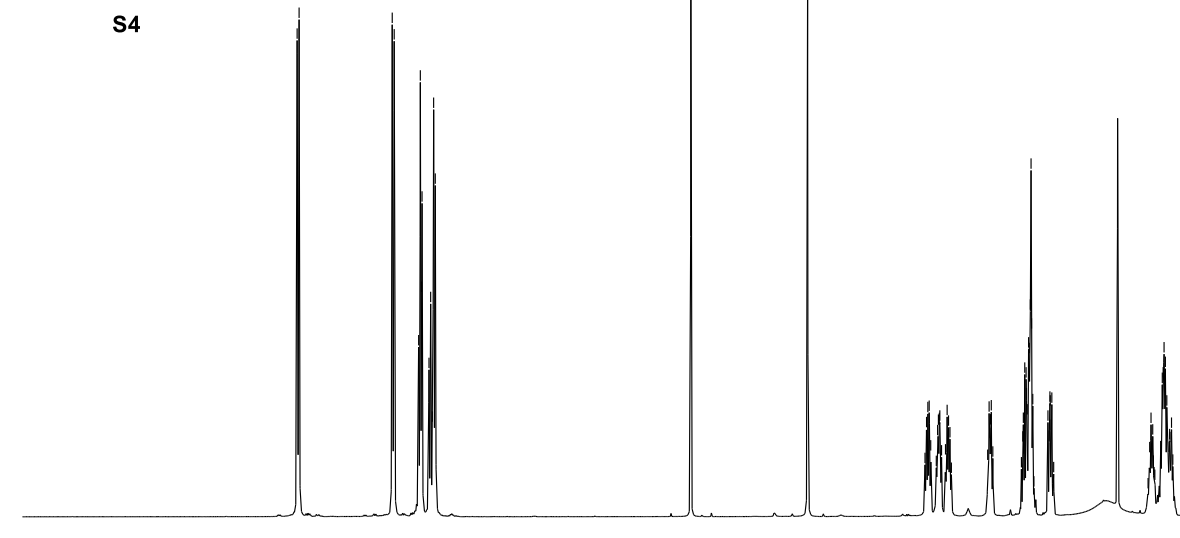

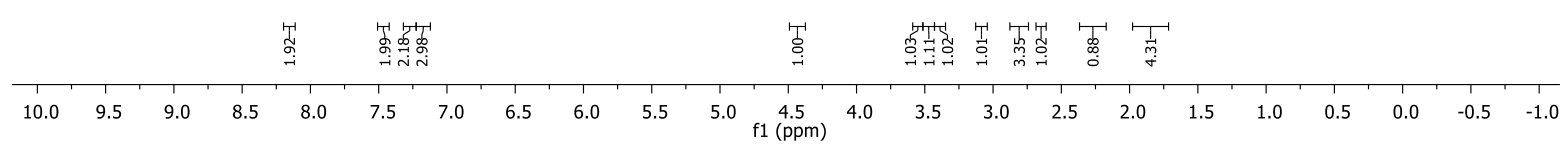

${ }^{13}$ C NMR (151 MHz, $\left.\mathrm{CDCl}_{3}\right)$<smiles>O=C(C(NCCc1ccccc1)c1ccc([N+](=O)[O-])cc1)N1CCCC1</smiles>

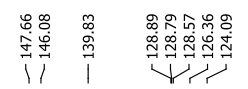

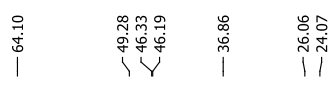

S4

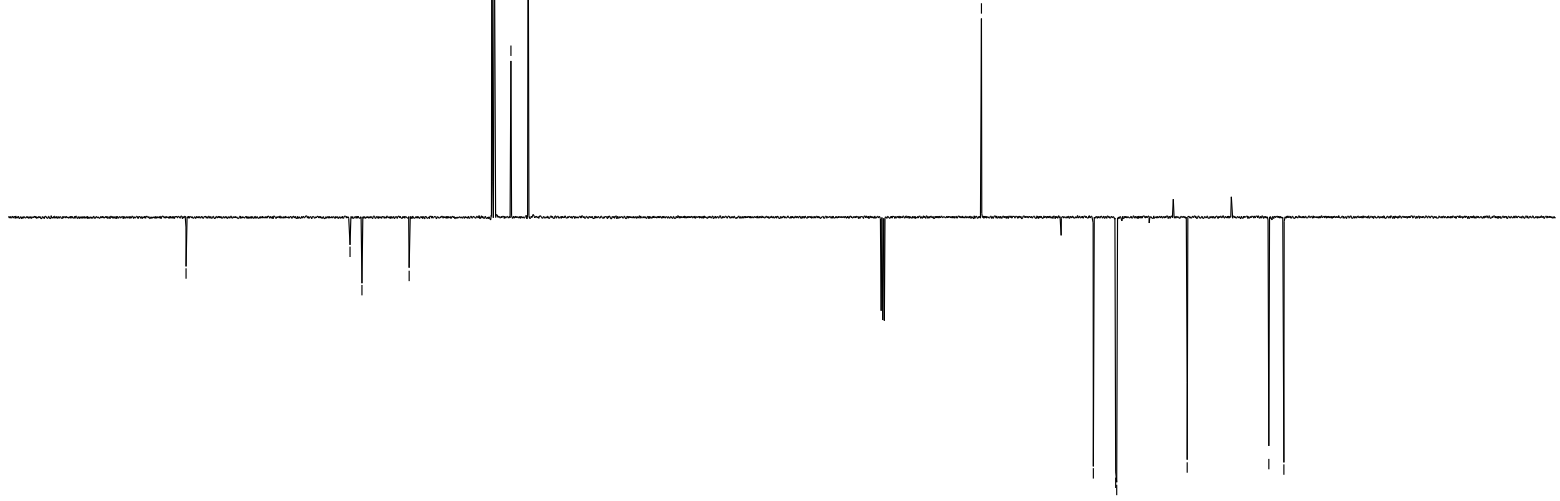


${ }^{1} \mathbf{H}$ NMR $\left(600 \mathrm{MHz}, \mathrm{CDCl}_{3}\right)$

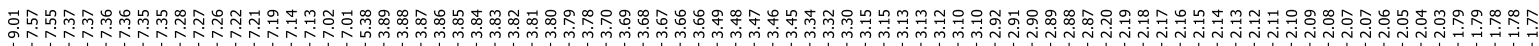

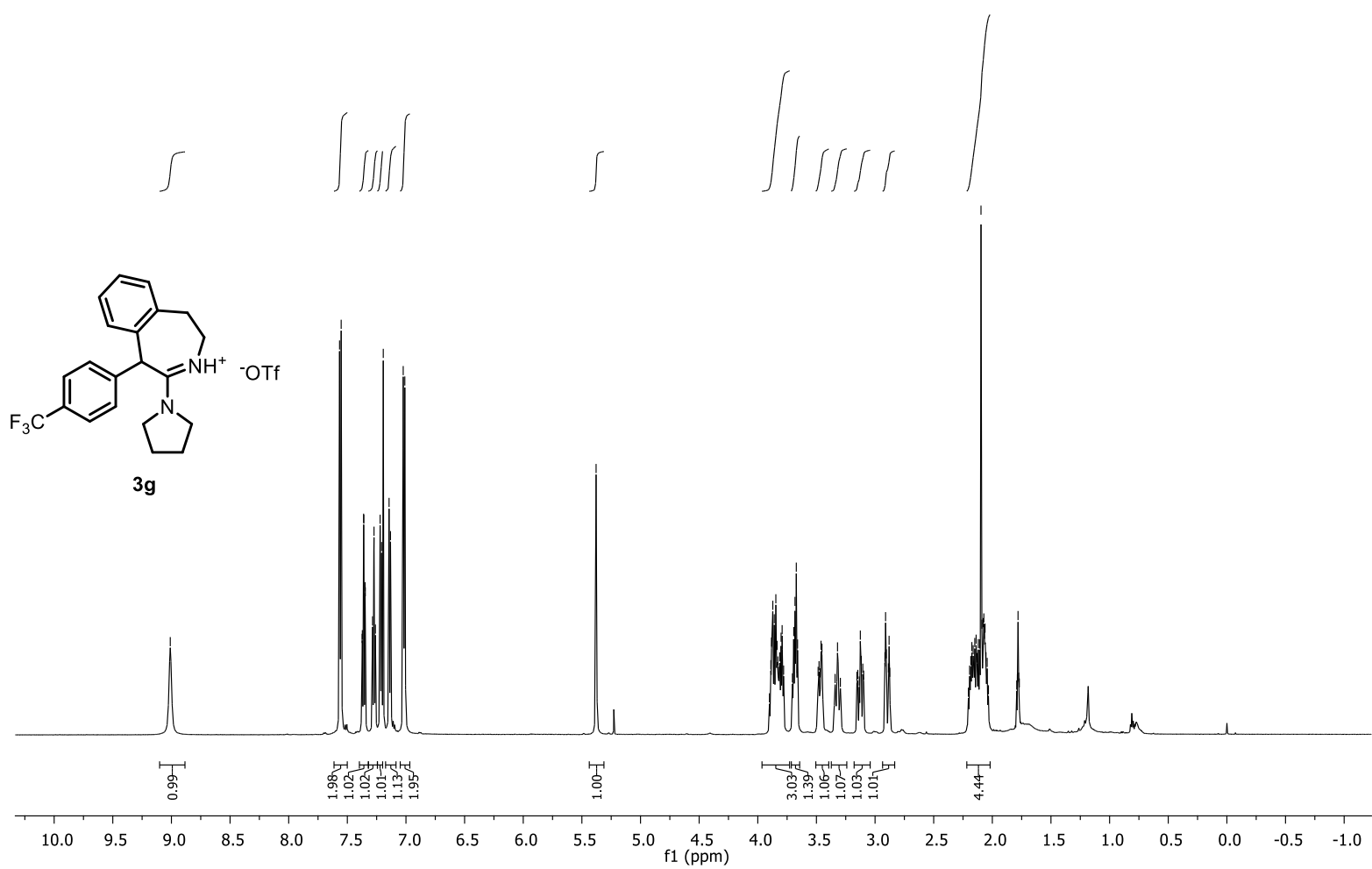

${ }^{13} \mathrm{C}$ NMR $\left(151 \mathrm{MHz}, \mathrm{CDCl}_{3}\right)$

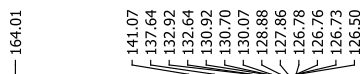

\丶j

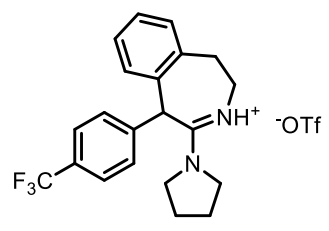

$3 g$

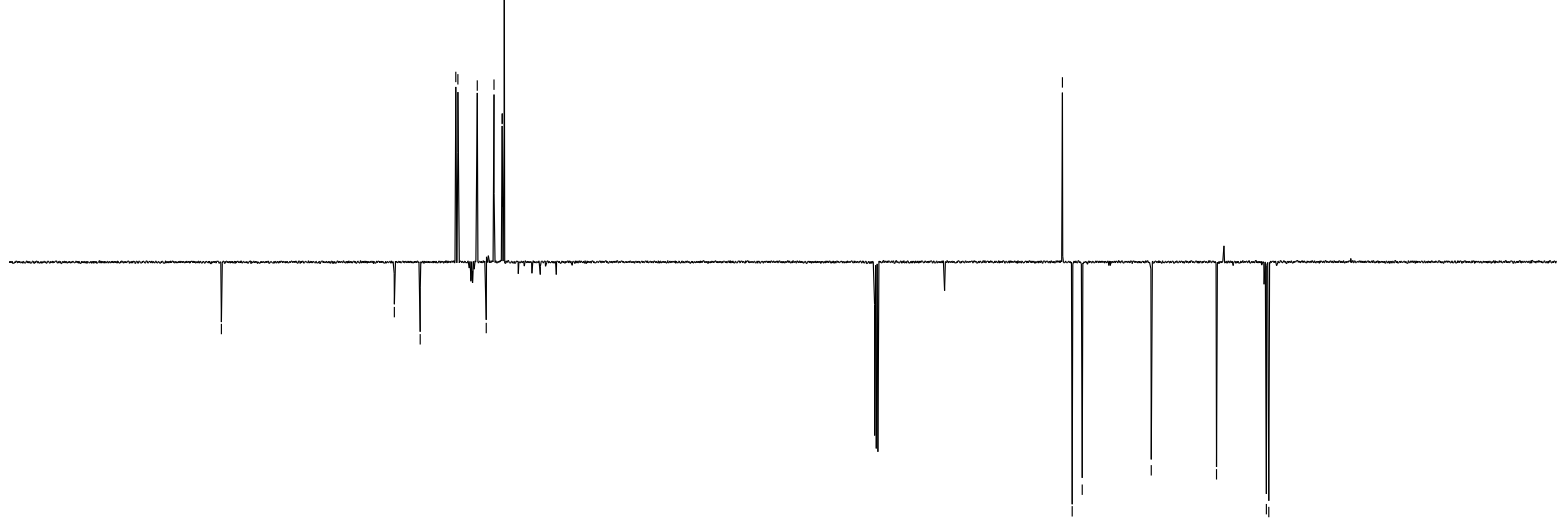


${ }^{19}$ F NMR (565 MHz, $\left.\mathrm{CDCl}_{3}\right)$
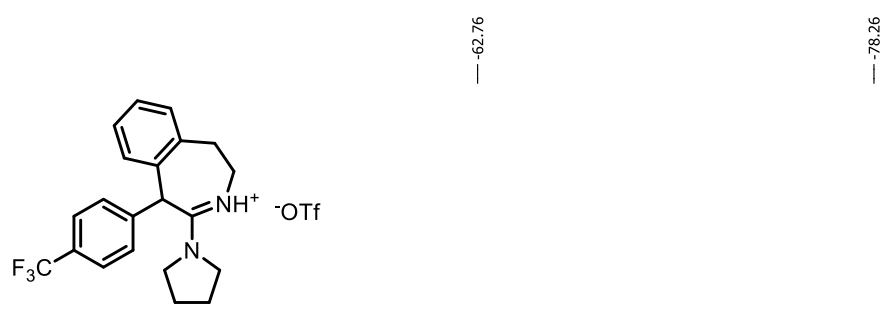

$3 \mathrm{~g}$

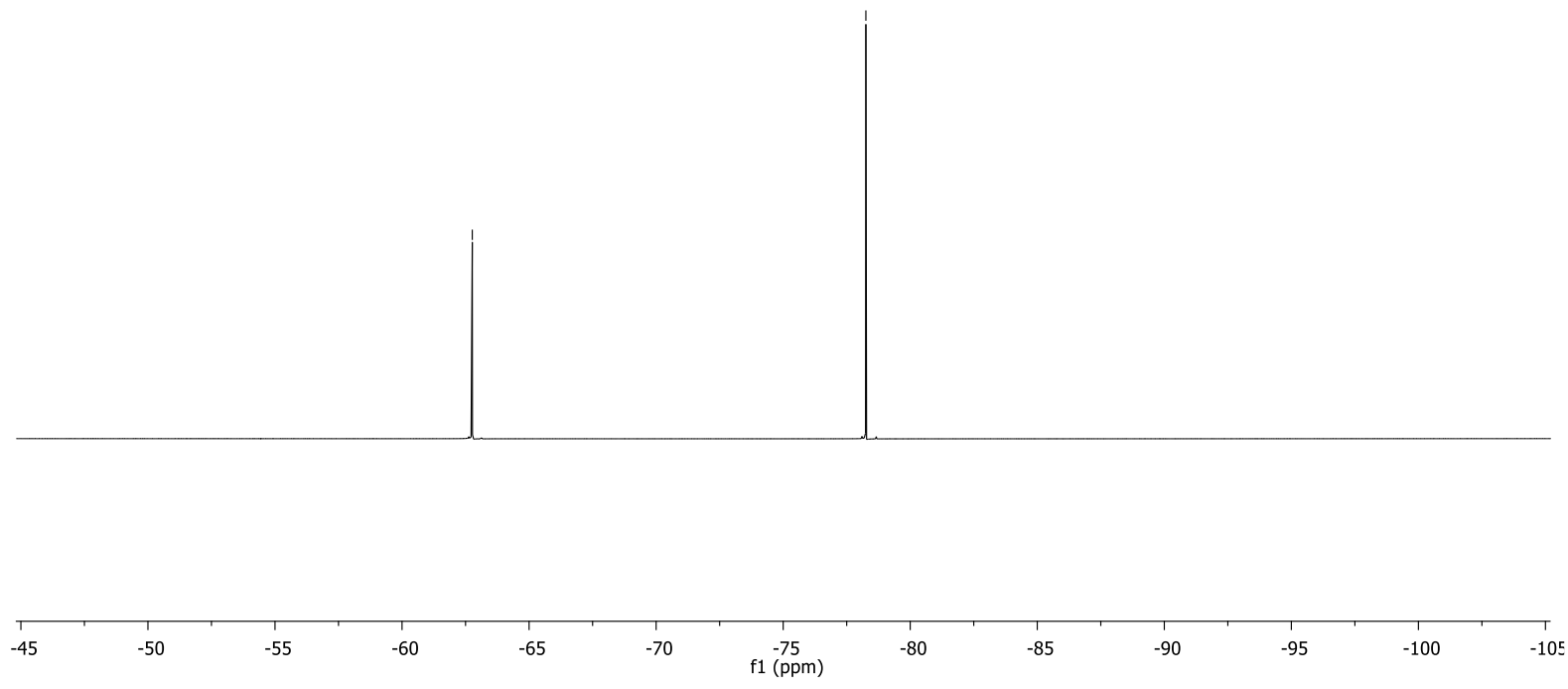

${ }^{1} \mathrm{H}$ NMR $\left(600 \mathrm{MHz}, \mathrm{CDCl}_{3}\right)$

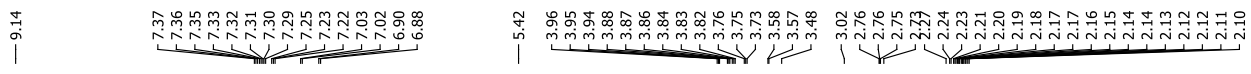
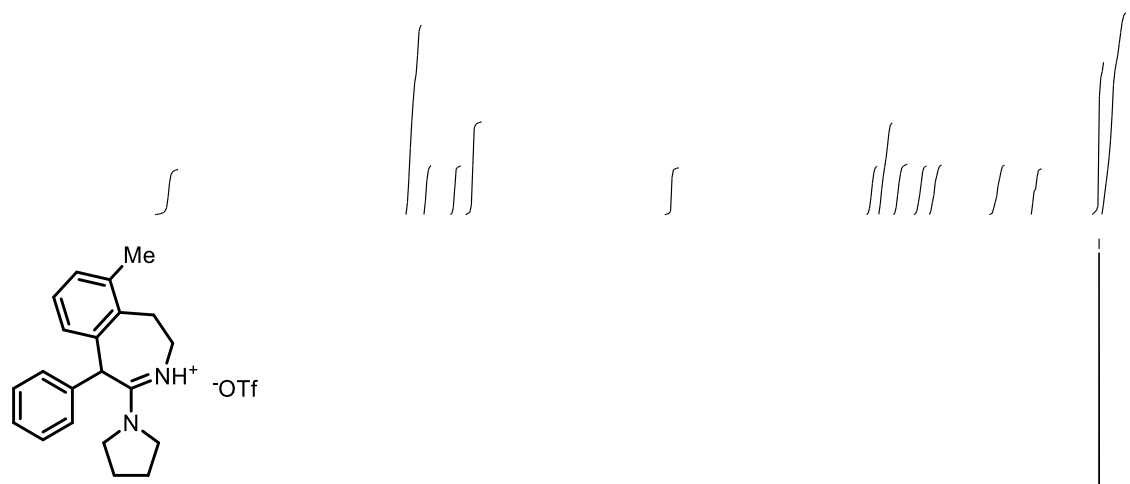

3h

ind it

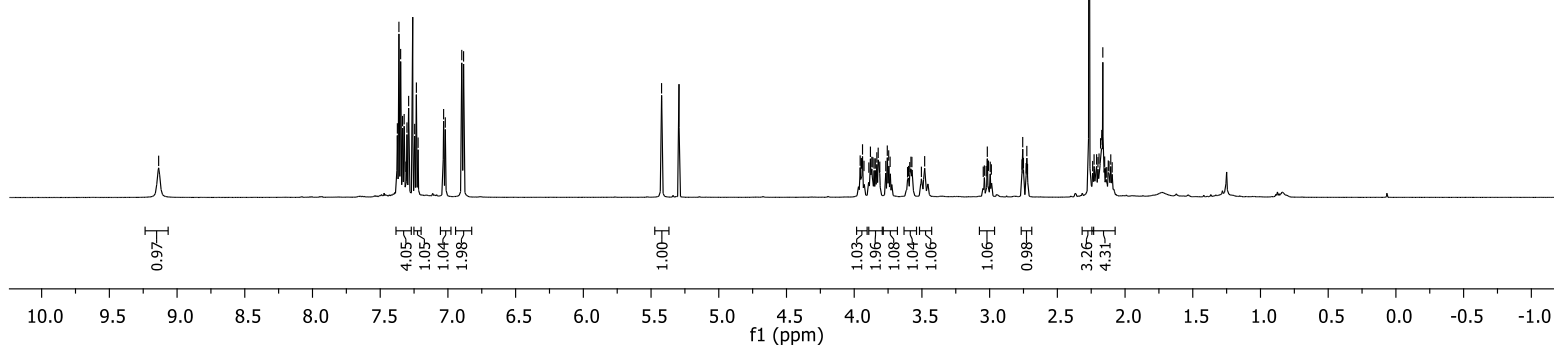


${ }^{13} \mathrm{C}$ NMR $\left(151 \mathrm{MHz}, \mathrm{CDCl}_{3}\right)$

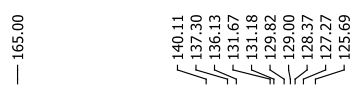

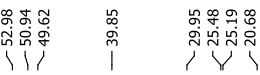
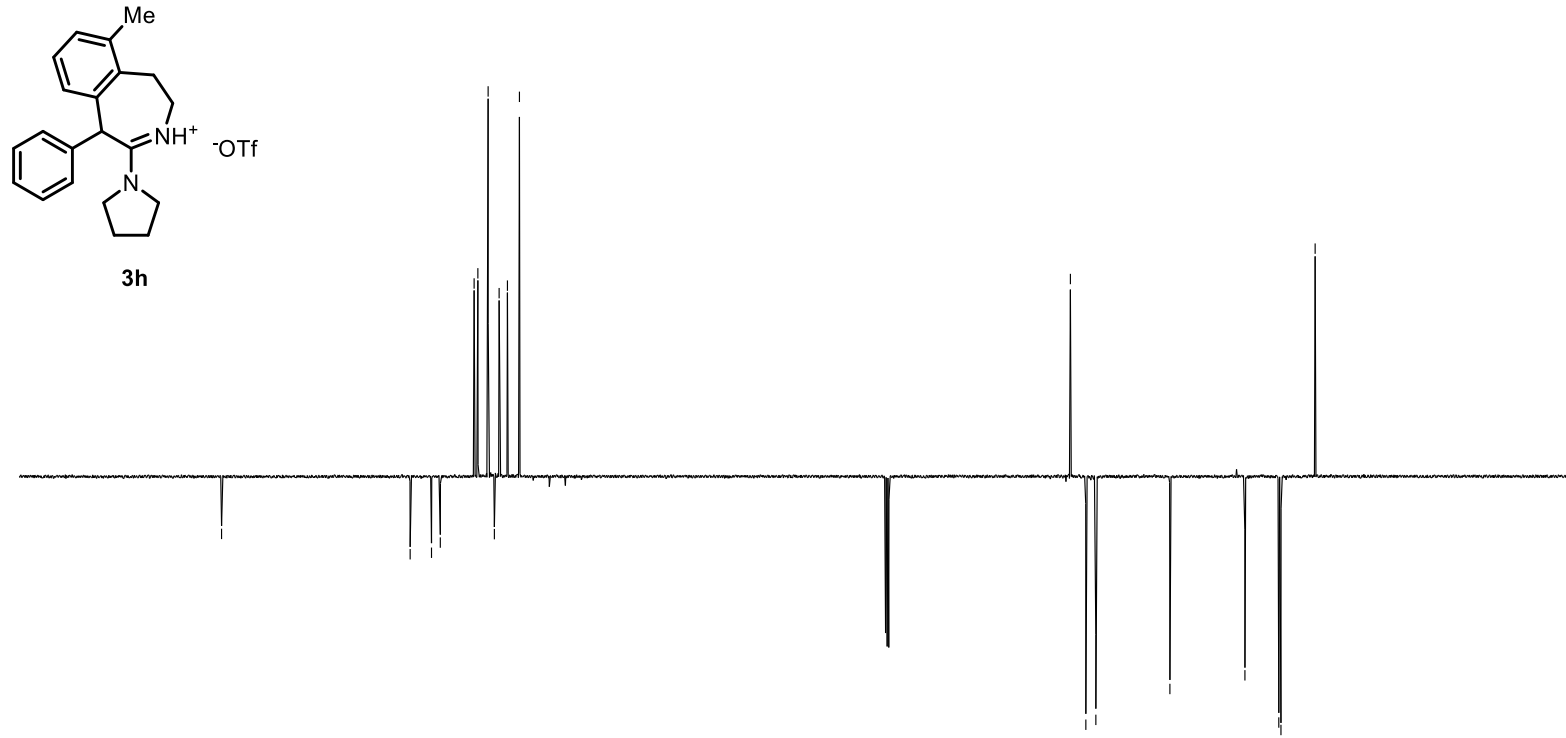

$\begin{array}{llllllllllllllllllllll}190 & 180 & 170 & 160 & 150 & 140 & 130 & 120 & 110 & 100 & \underset{\mathrm{f} 1}{9(\mathrm{ppm})} & 80 & 70 & 60 & 50 & 40 & 30 & 20 & 10 & 0 & -10\end{array}$

${ }^{19} \mathrm{~F}$ NMR $\left(565 \mathrm{MHz}, \mathrm{CDCl}_{3}\right)$

$$
\underset{\substack{\text { } \\ \stackrel{1}{i}}}{1}
$$<smiles>Cc1cccc2c1CC[NH+]=C(N1CCCC1)C2c1ccccc1</smiles>

3h

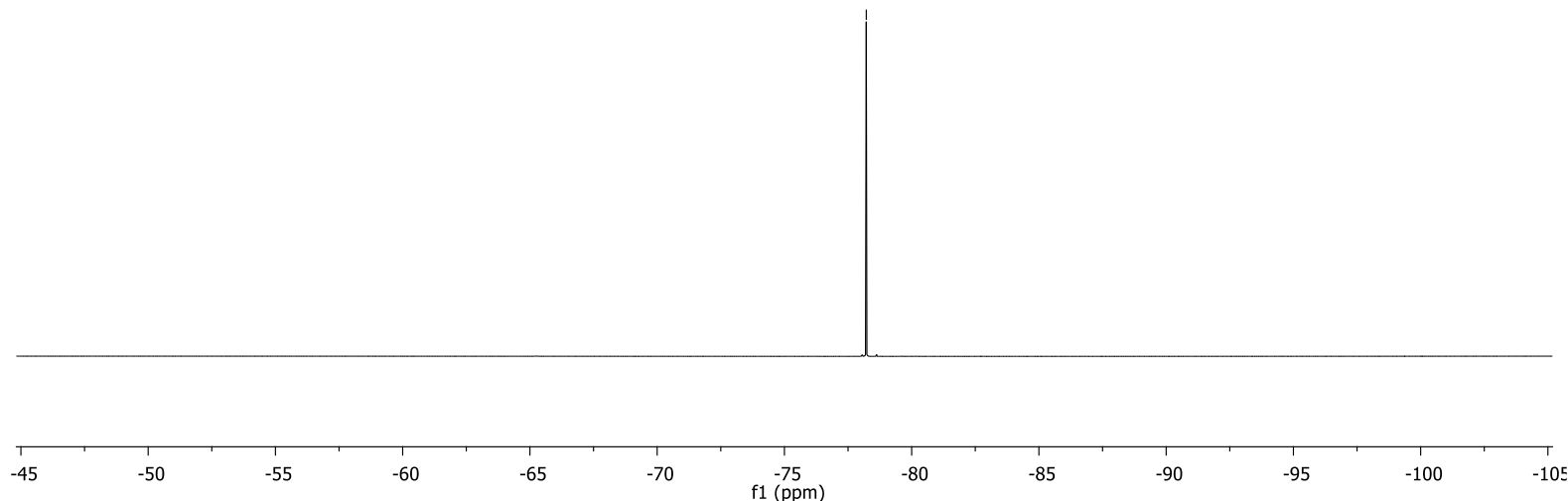


${ }^{1} \mathbf{H}$ NMR $\left(600 \mathrm{MHz}, \mathrm{CDCl}_{3}\right)$

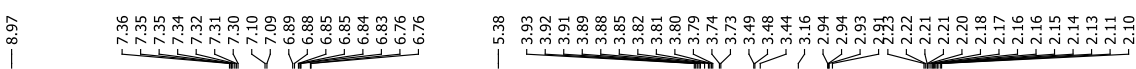

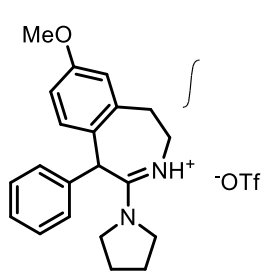<smiles>C1#CCCC#C1</smiles><smiles>CC#CC(C)CCCC</smiles>

$3 \mathbf{i}$

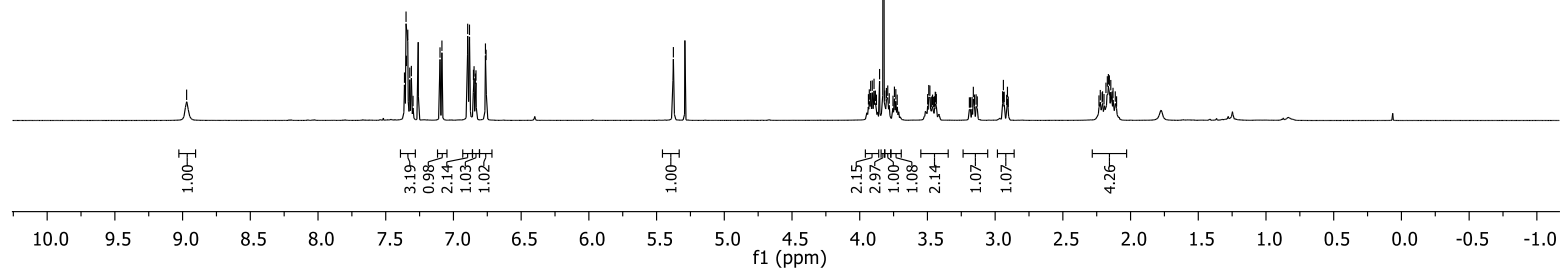

${ }^{13} \mathrm{C}$ NMR (151 MHz, $\left.\mathrm{CDCl}_{3}\right)$

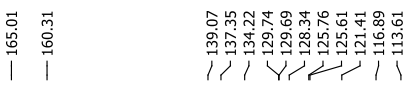

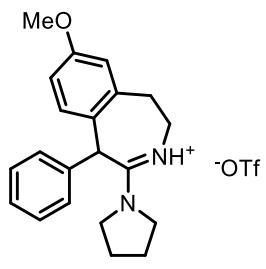

3i

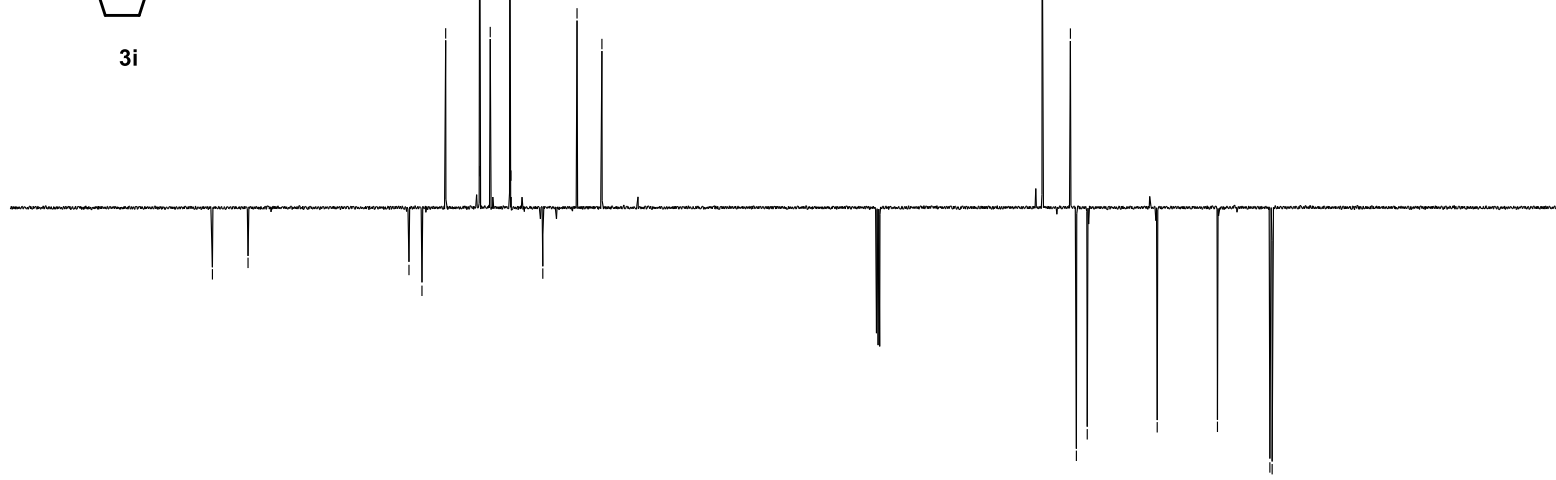

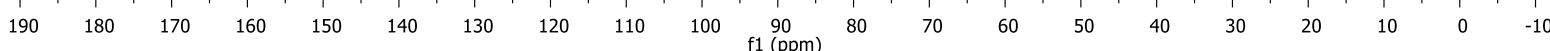


${ }^{19}$ F NMR $\left(565 \mathrm{MHz}, \mathrm{CDCl}_{3}\right)$

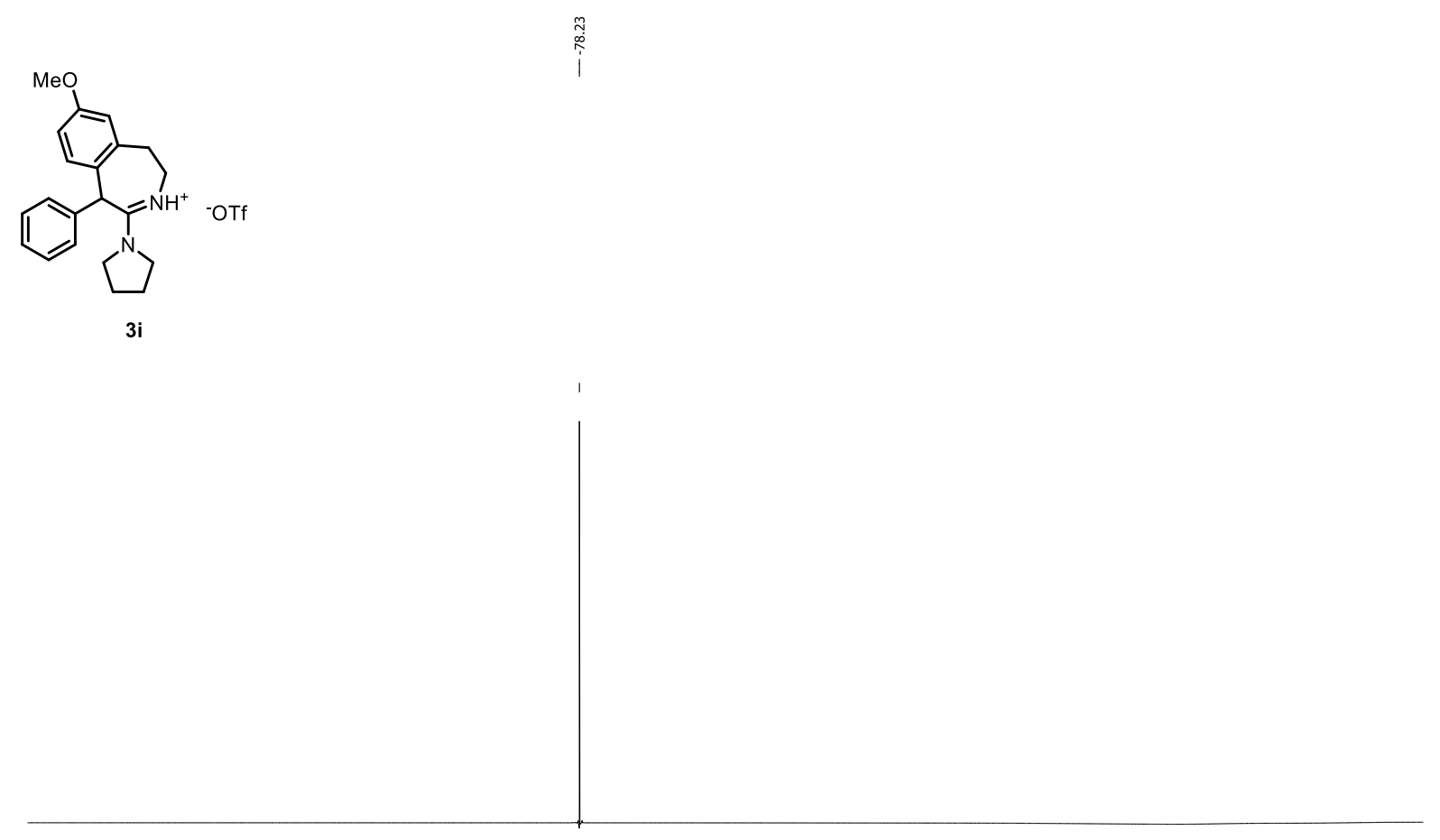

\begin{tabular}{lllllllllllllllllllllllllllll}
\hline & 0 & -10 & -20 & -30 & -40 & -50 & -60 & -70 & -80 & -90 & -100 & -110 & -120 & -130 & -140 & -150 & -160 & -170 & -180 & -190 & -200
\end{tabular}

${ }^{1} \mathbf{H}$ NMR (600 MHz, $\left.\mathrm{CDCl}_{3}\right)$

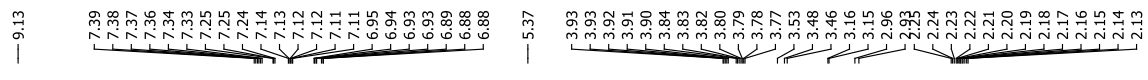

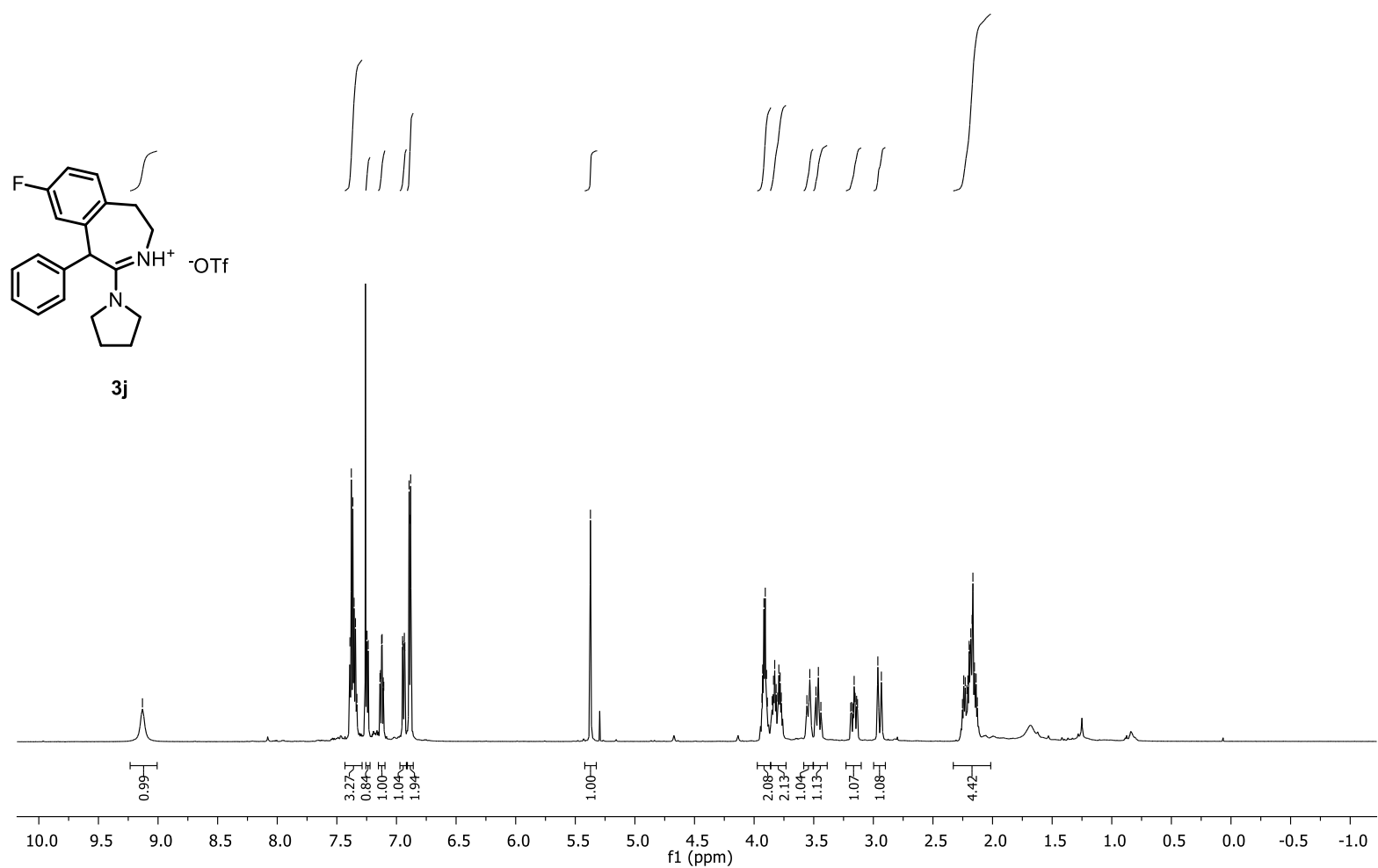


${ }^{13} \mathrm{C}$ NMR $\left(151 \mathrm{MHz}, \mathrm{CDCl}_{3}\right)$

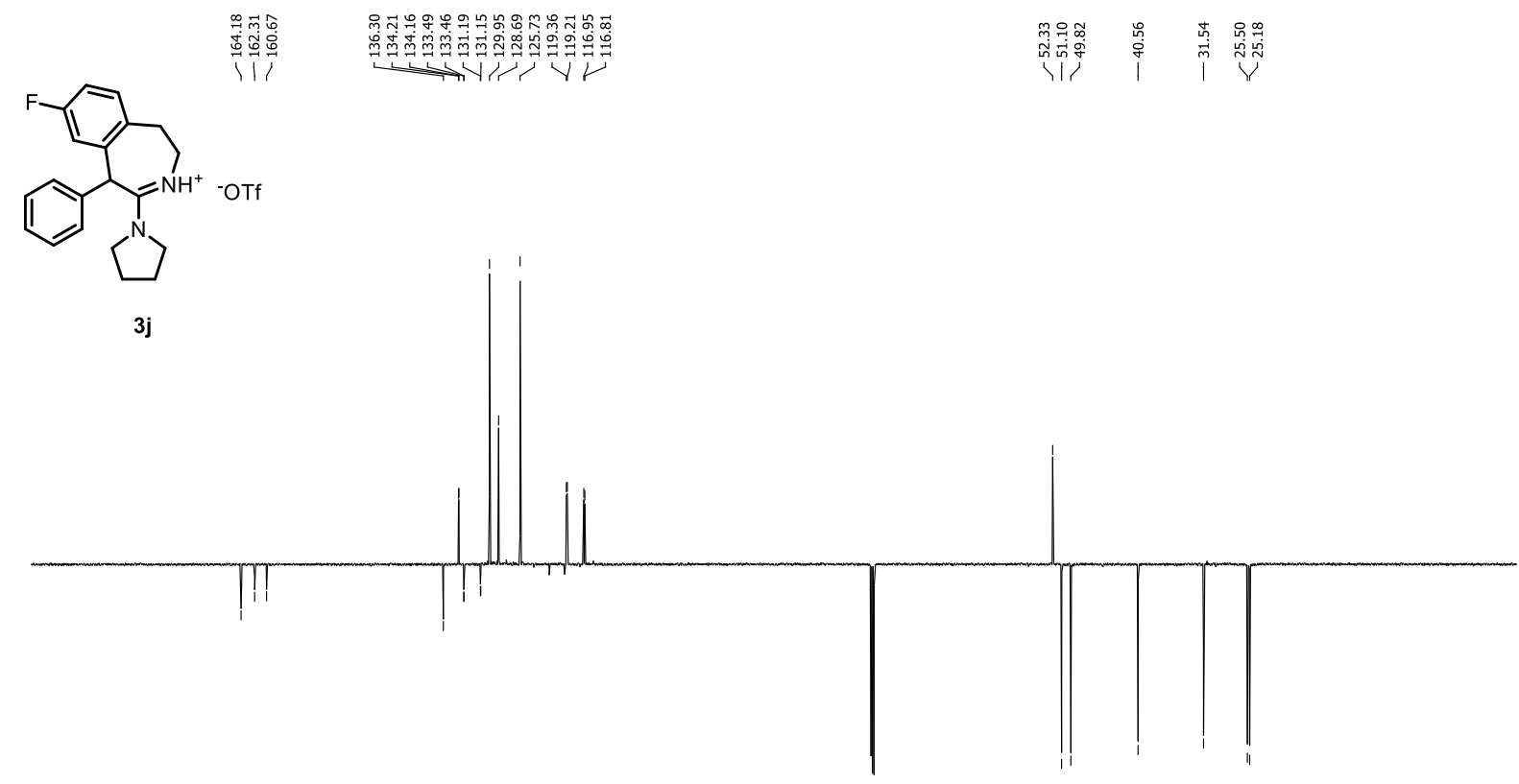

\begin{tabular}{lllllllllllllllllllllllllllll}
\hline 190 & 180 & 170 & 160 & 150 & 140 & 130 & 120 & 110 & 100 & $\begin{array}{c}90 \\
\mathrm{f} 1(\mathrm{ppm})\end{array}$ & 80 & 70 & 60 & 50 & 40 & 30 & 20 & 10 & 0 & -10
\end{tabular}

${ }^{19}$ F NMR $\left(565 \mathrm{MHz}, \mathrm{CDCl}_{3}\right)$
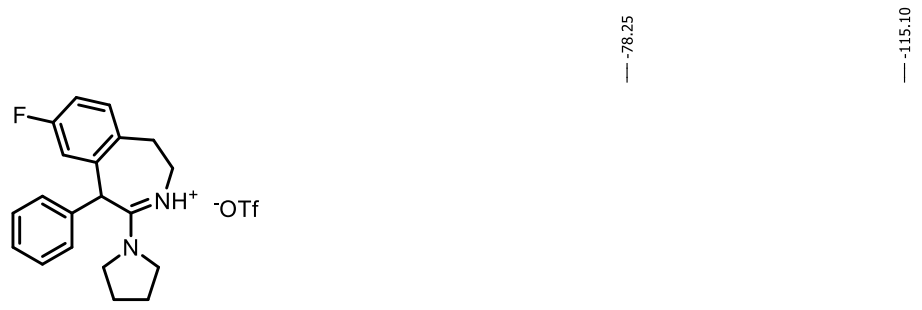

3j

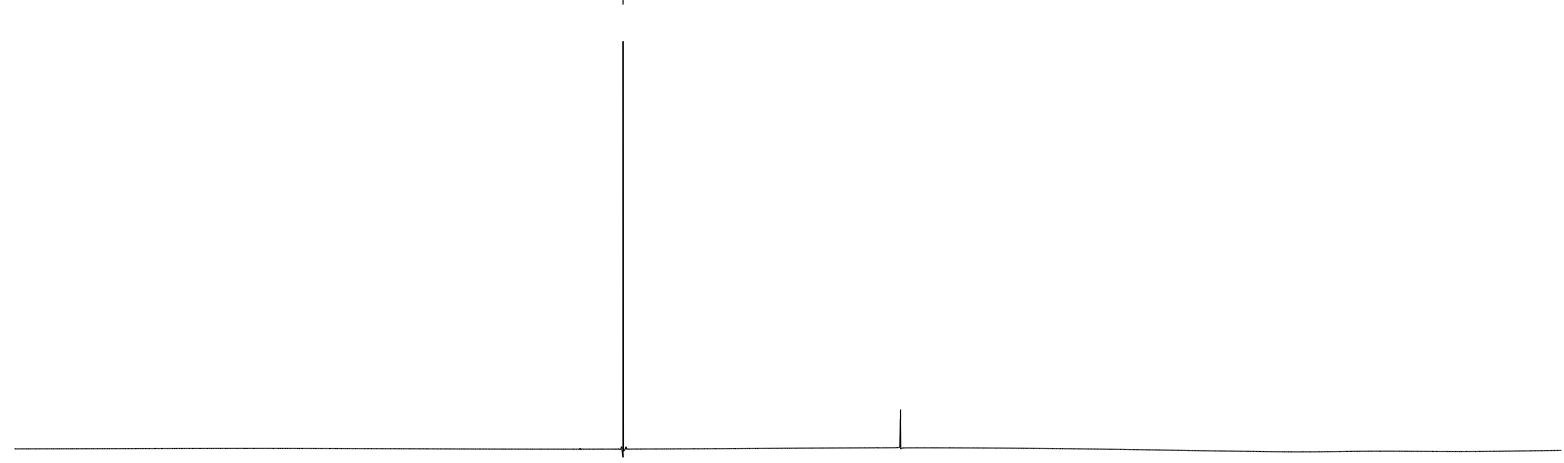

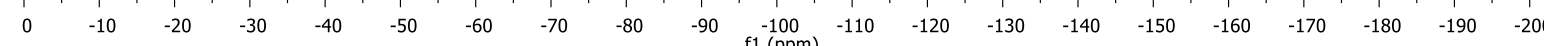


${ }^{1} \mathrm{H}$ NMR (600 MHz, $\left.\mathrm{CDCl}_{3}\right)$

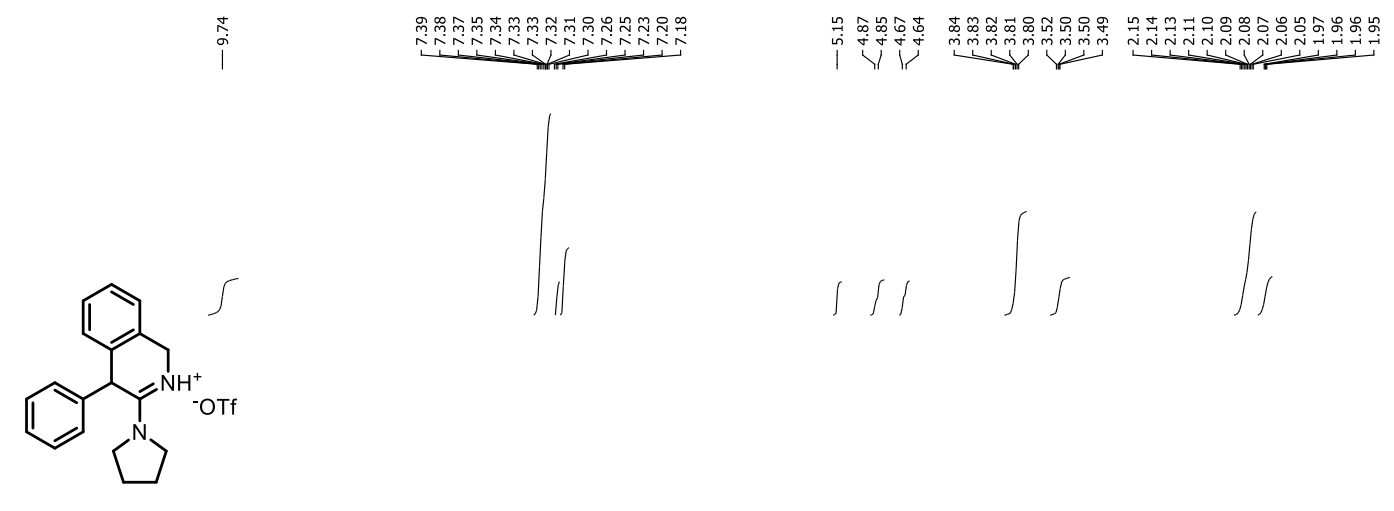

3I

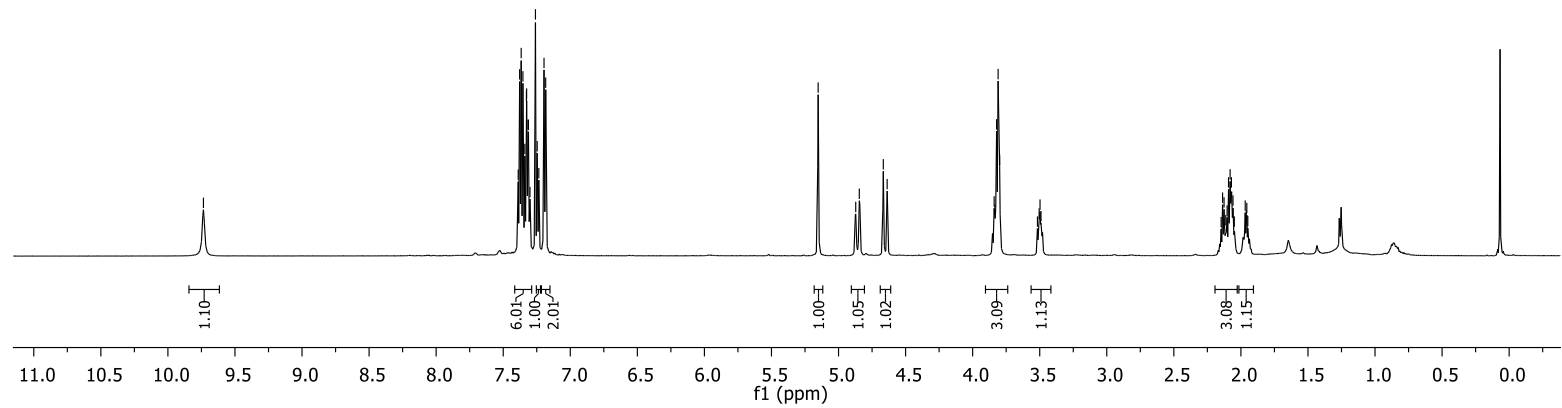

${ }^{13}$ C NMR (151 MHz, $\left.\mathrm{CDCl}_{3}\right)$

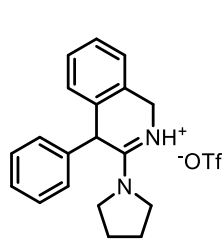

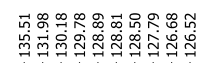

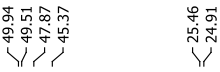

31

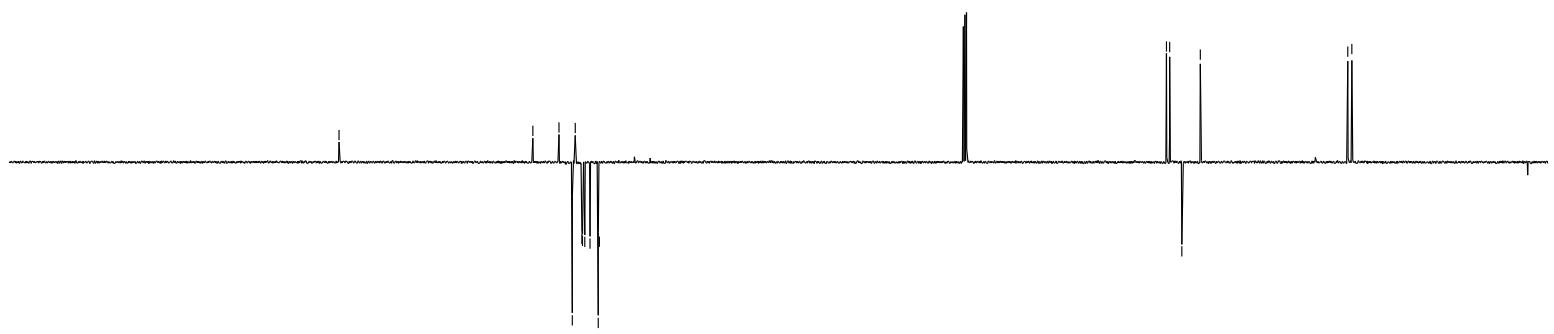

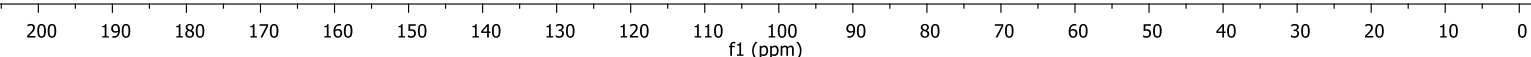


${ }^{19}$ F NMR $\left(565 \mathrm{MHz}, \mathrm{CDCl}_{3}\right)$

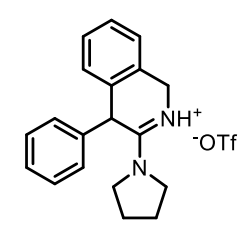

31

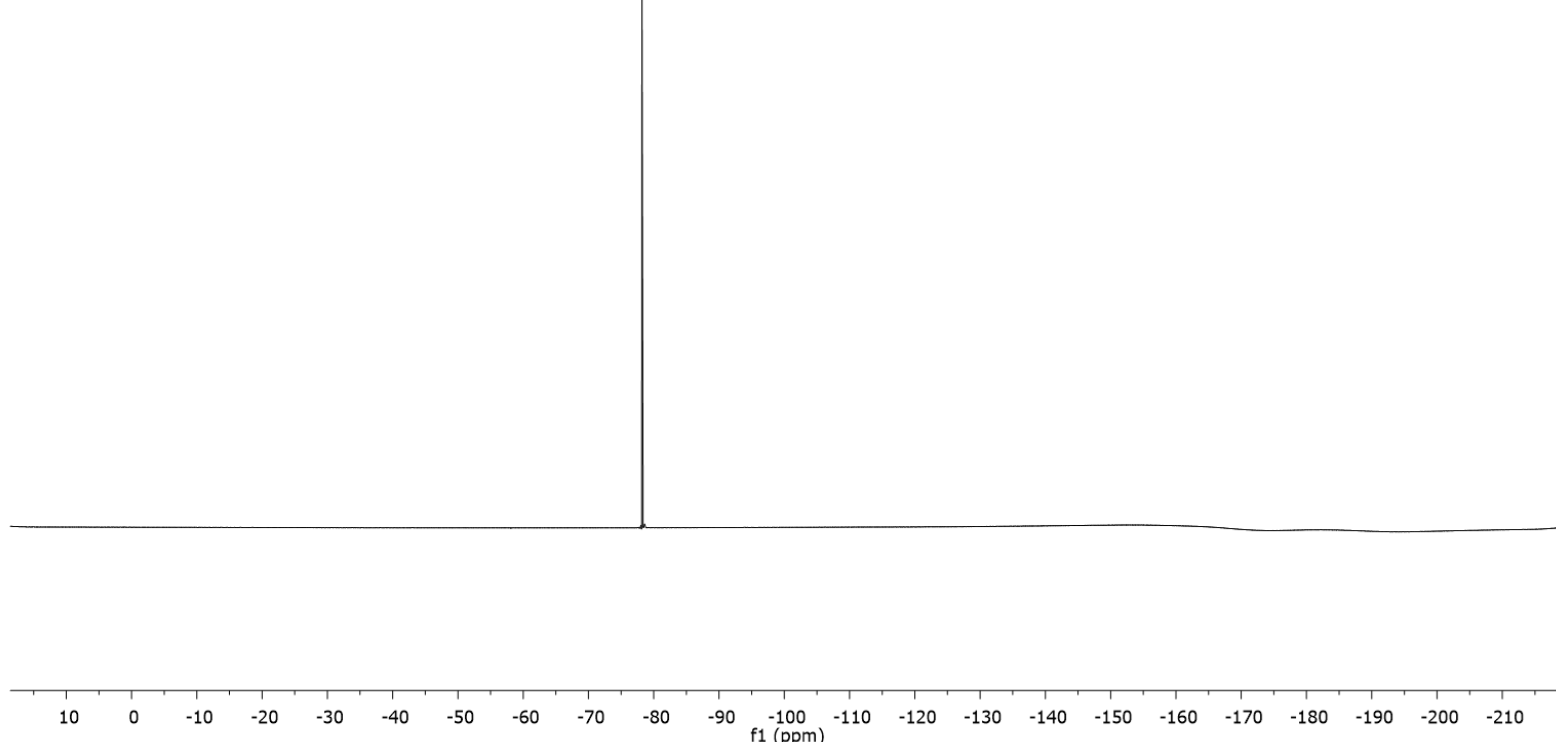

${ }^{1} \mathbf{H} \mathbf{N M R}\left(600 \mathrm{MHz}, \mathrm{CDCl}_{3}\right)$

年<smiles>COc1ccc2c(c1)C[NH+]([O-])C(N1CCCC1)C2c1ccccc1</smiles>

$3 m$
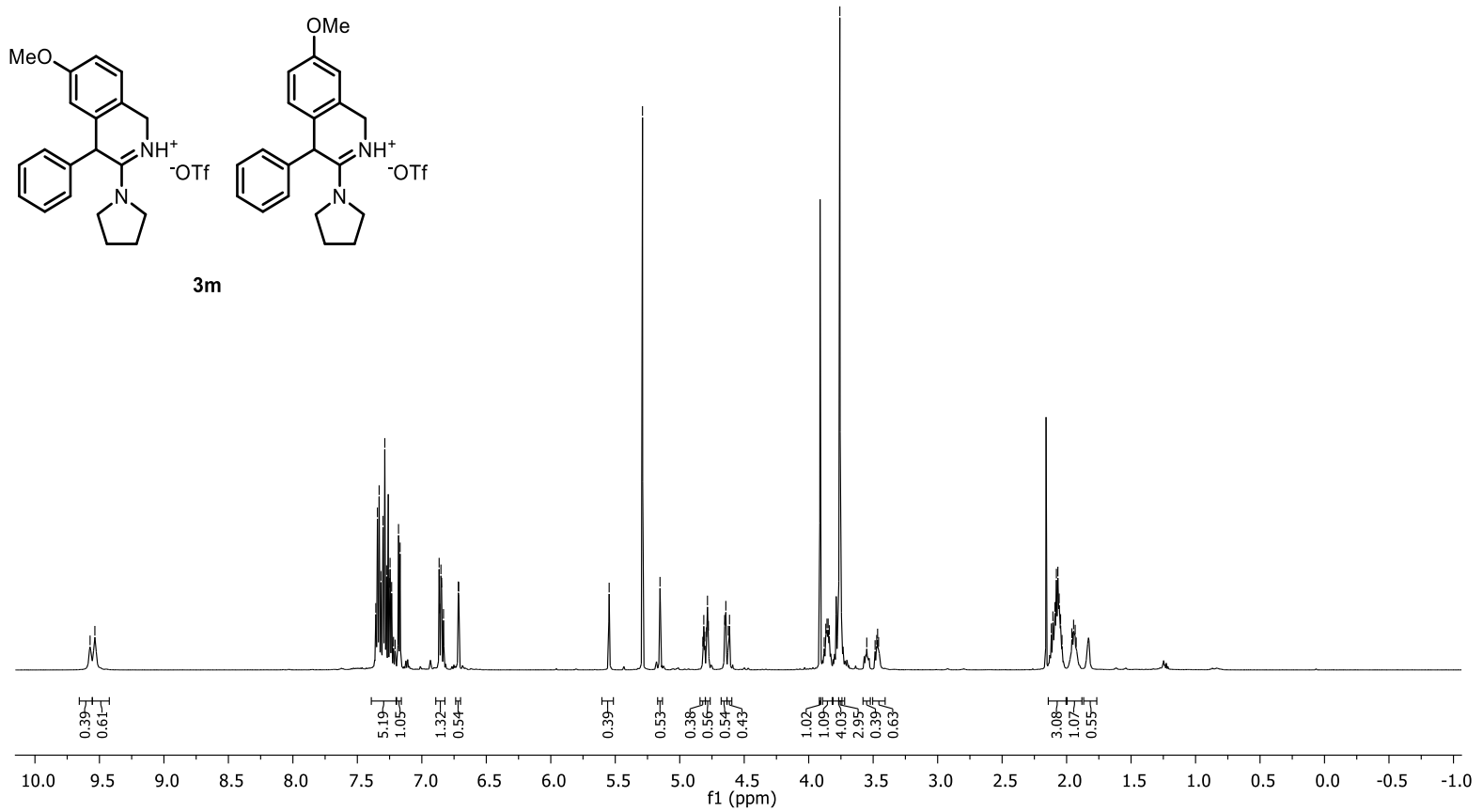
${ }^{13} \mathrm{C}$ NMR (151 MHz, $\left.\mathrm{CDCl}_{3}\right)$

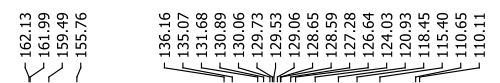

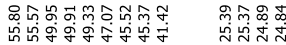

पर

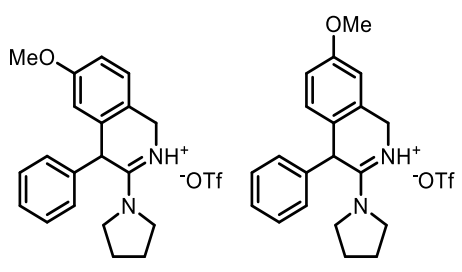

$3 m$

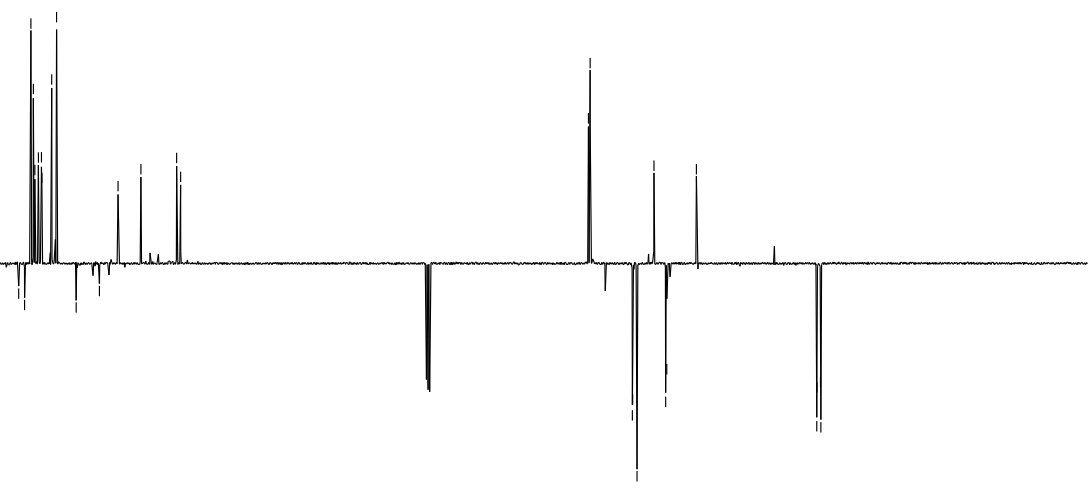

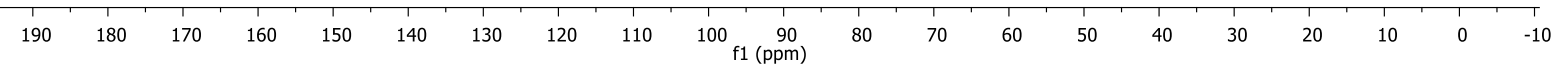

${ }^{19} \mathrm{~F}$ NMR $\left(565 \mathrm{MHz}, \mathrm{CDCl}_{3}\right)$

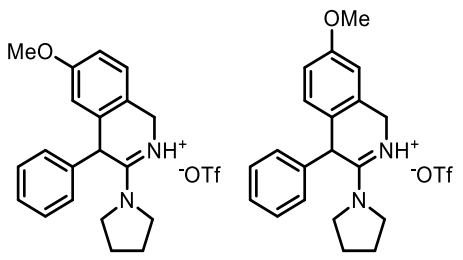

$3 m$

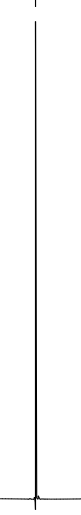

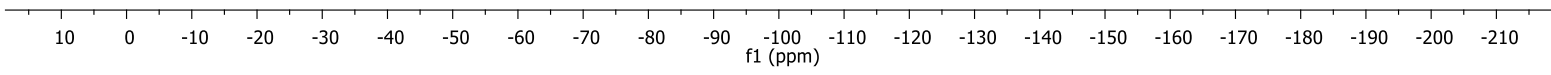


${ }^{1} \mathbf{H}$ NMR $\left(600 \mathrm{MHz}, \mathrm{CDCl}_{3}\right)$

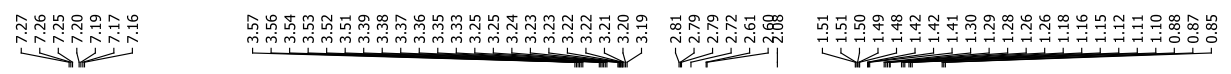<smiles>CCC(NCCc1ccccc1)C(=O)N(CC)CC</smiles>

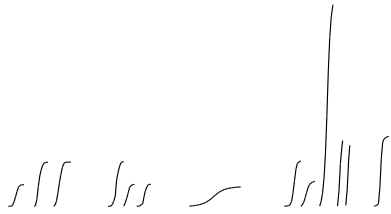

s5
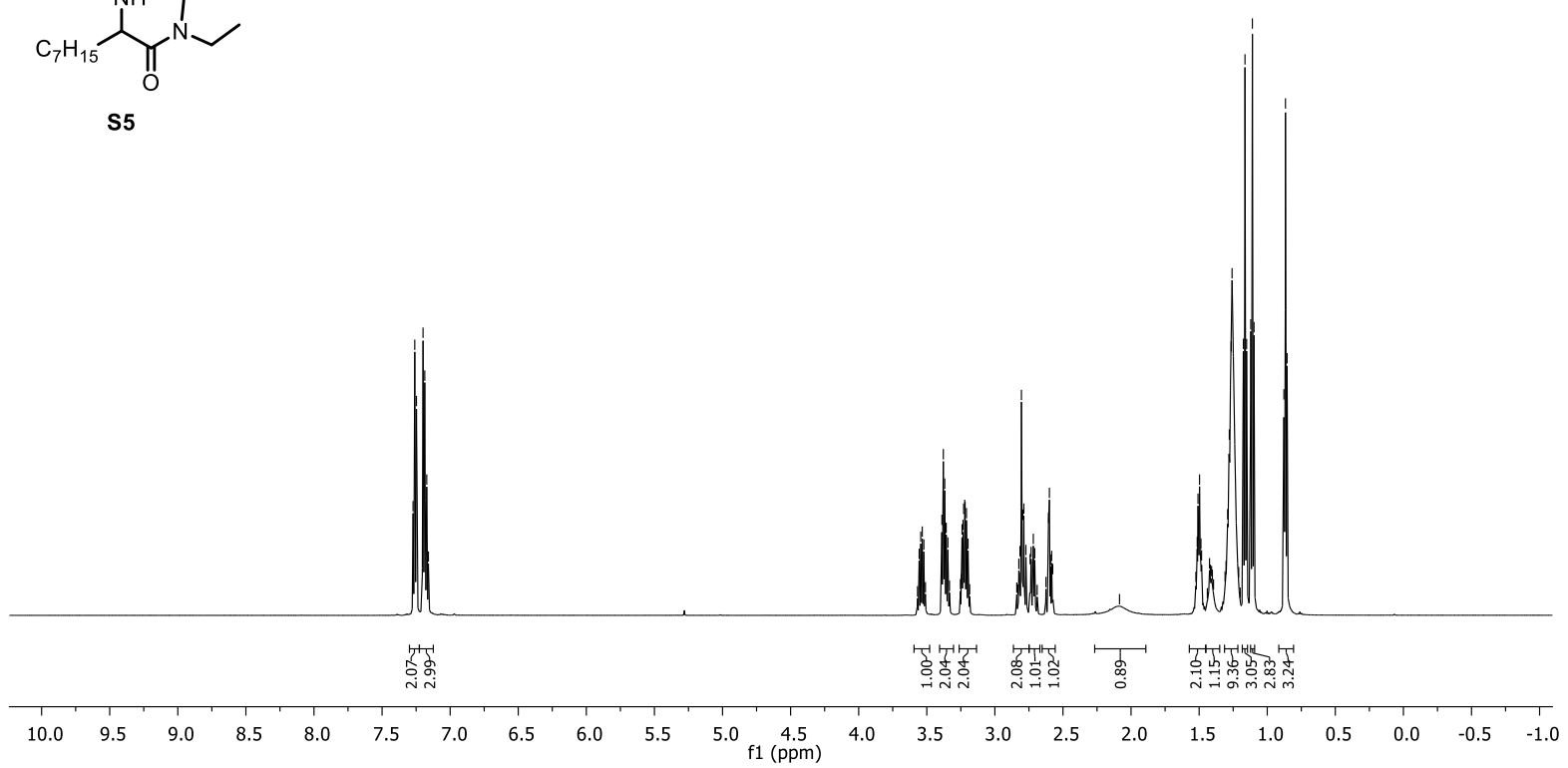

${ }^{13} \mathrm{C}$ NMR (151 MHz, $\left.\mathrm{CDCl}_{3}\right)$

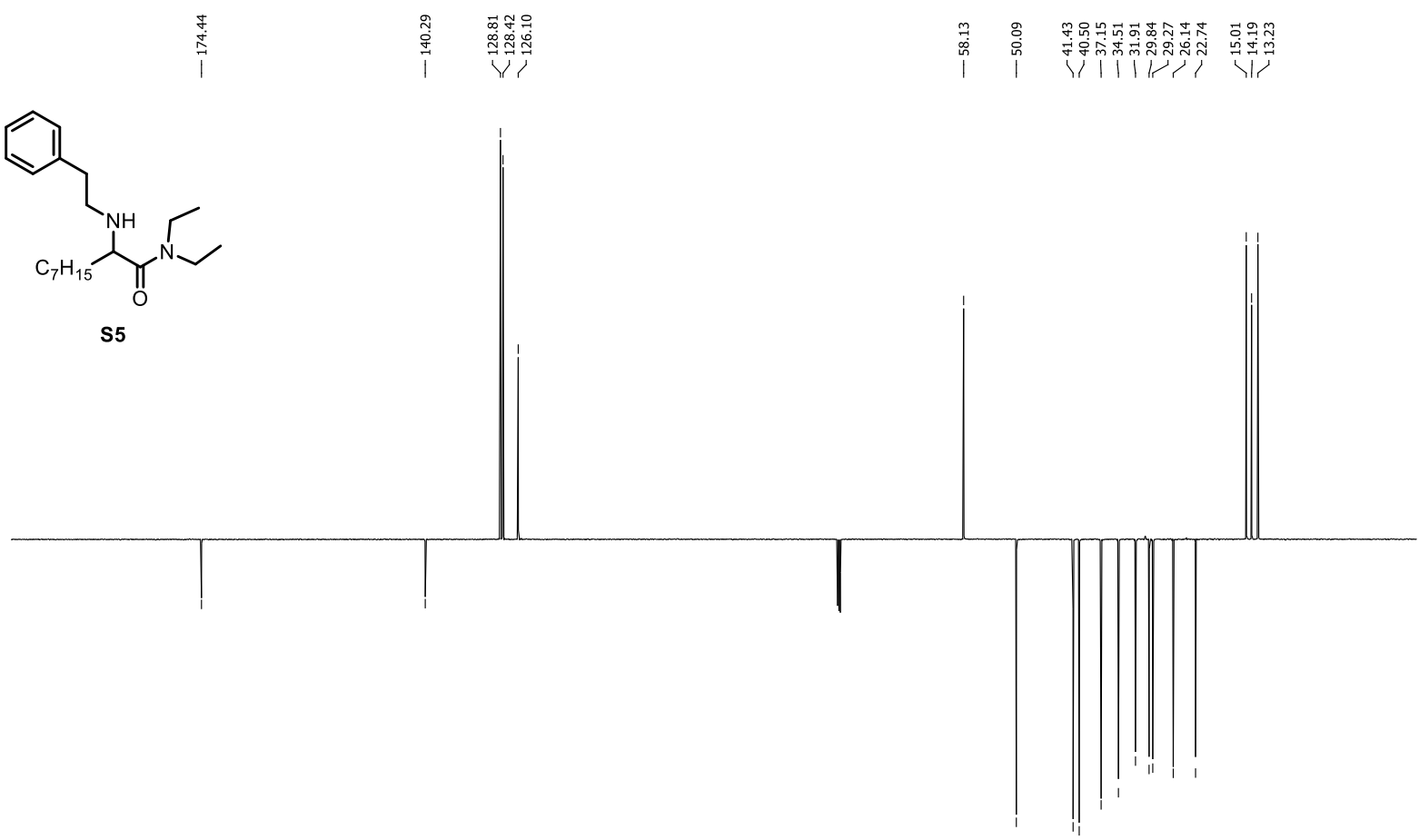

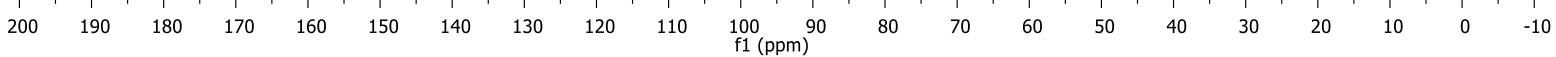


${ }^{1} \mathbf{H}$ NMR $\left(600 \mathrm{MHz}, \mathrm{CDCl}_{3}\right)$

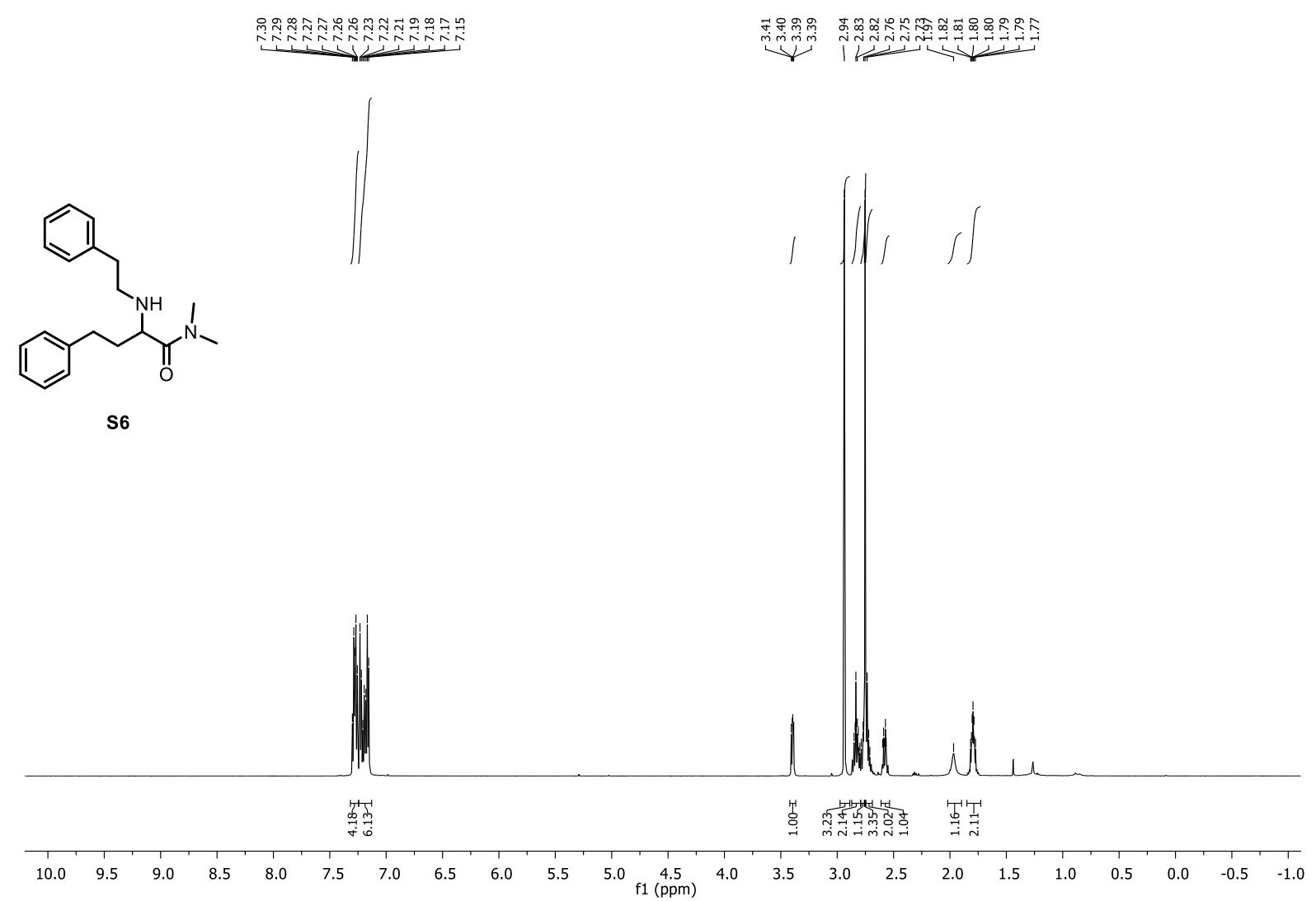

${ }^{13}$ C NMR (151 MHz, $\left.\mathrm{CDCl}_{3}\right)$
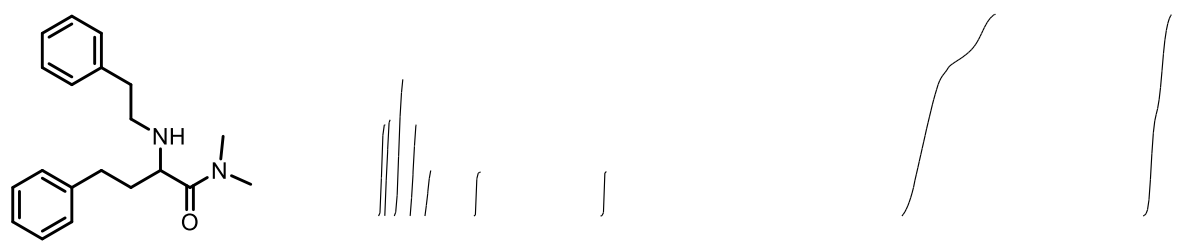

s6

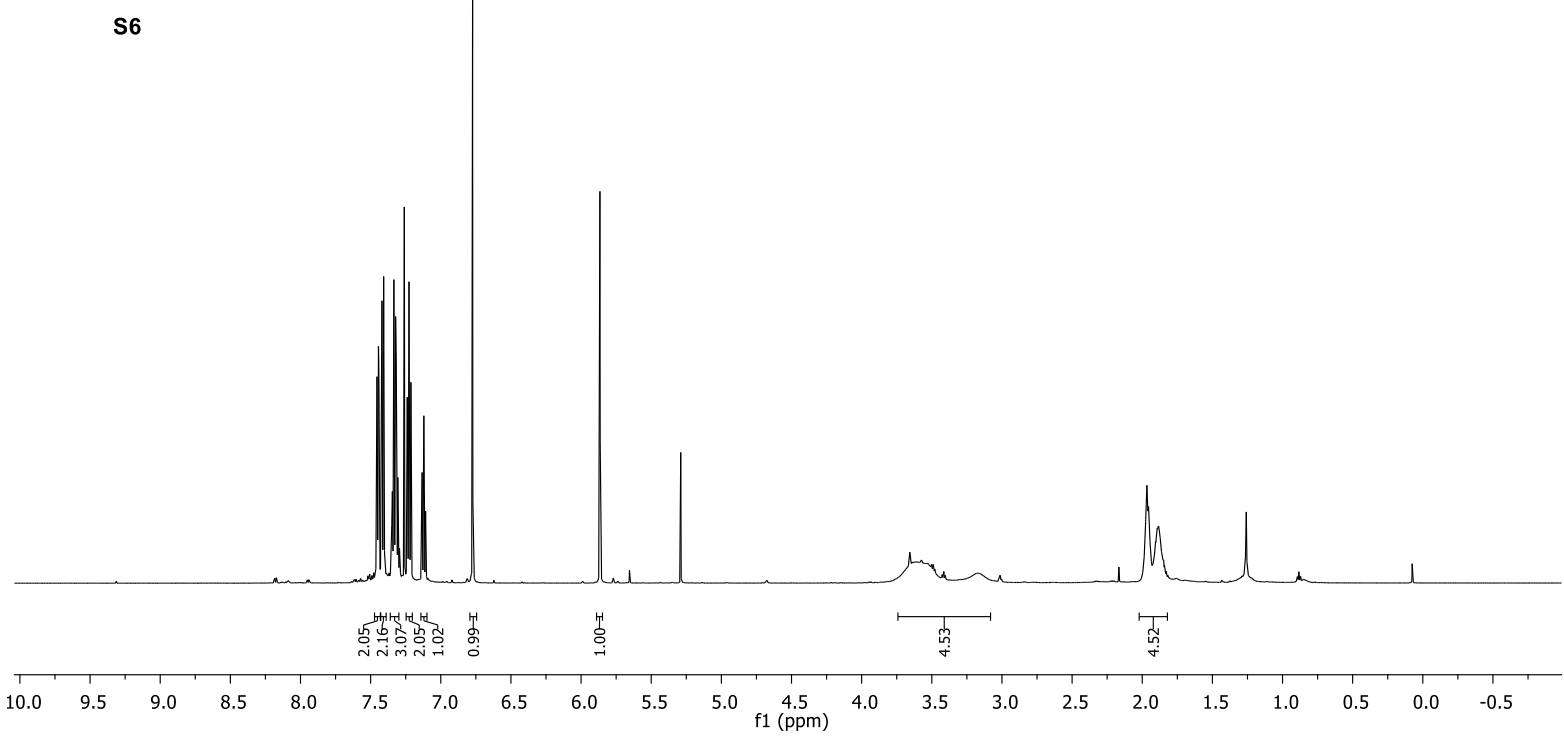


${ }^{1} \mathrm{H}$ NMR $\left(600 \mathrm{MHz}, \mathrm{CDCl}_{3}\right)$

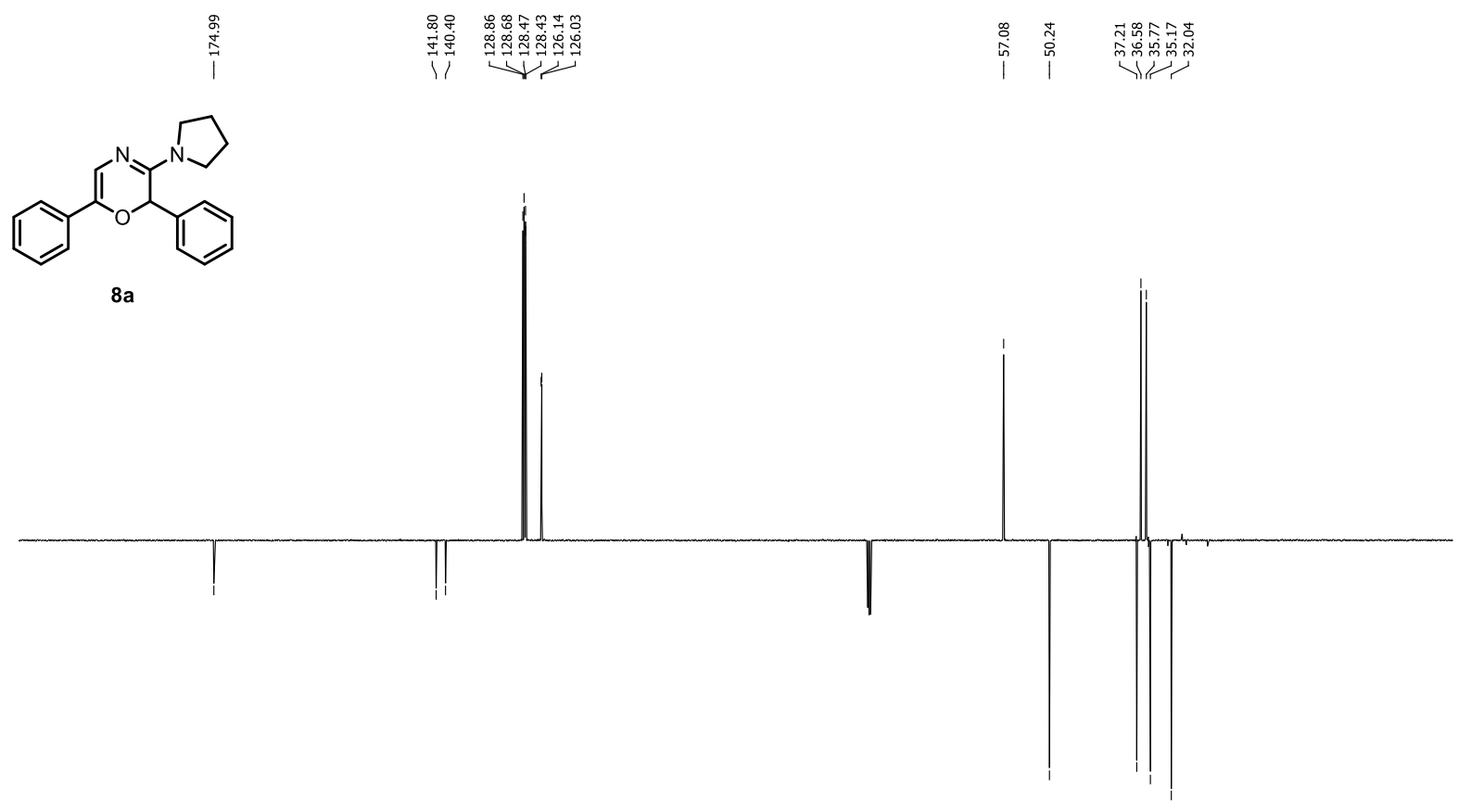

\begin{tabular}{llllllllllllllllllllllll}
\hline & 190 & 180 & 170 & 160 & 150 & 140 & 130 & 120 & 110 & $\underset{\mathrm{f} 1(\mathrm{ppm})}{100}$ & 80 & 70 & 60 & 50 & 40 & 30 & 20 & 10 & 0
\end{tabular}

${ }^{13} \mathrm{C}$ NMR $\left(151 \mathrm{MHz}, \mathrm{CDCl}_{3}\right)$
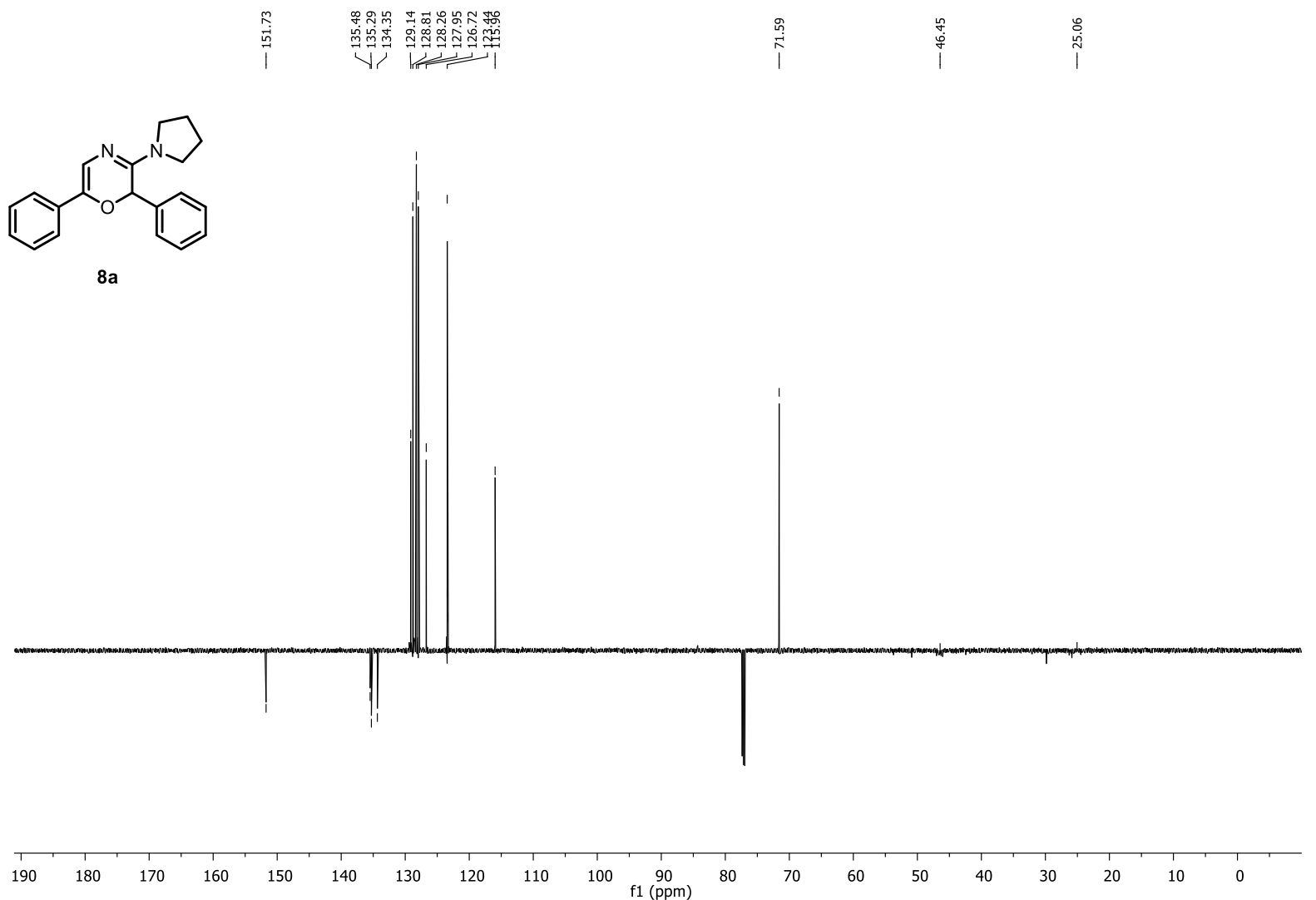
HSQC

${ }^{1} \mathbf{H}$ NMR $\left(600 \mathrm{MHz}, \mathrm{CDCl}_{3}\right),{ }^{13} \mathrm{C}$ NMR (151 MHz, CDCl 3 )
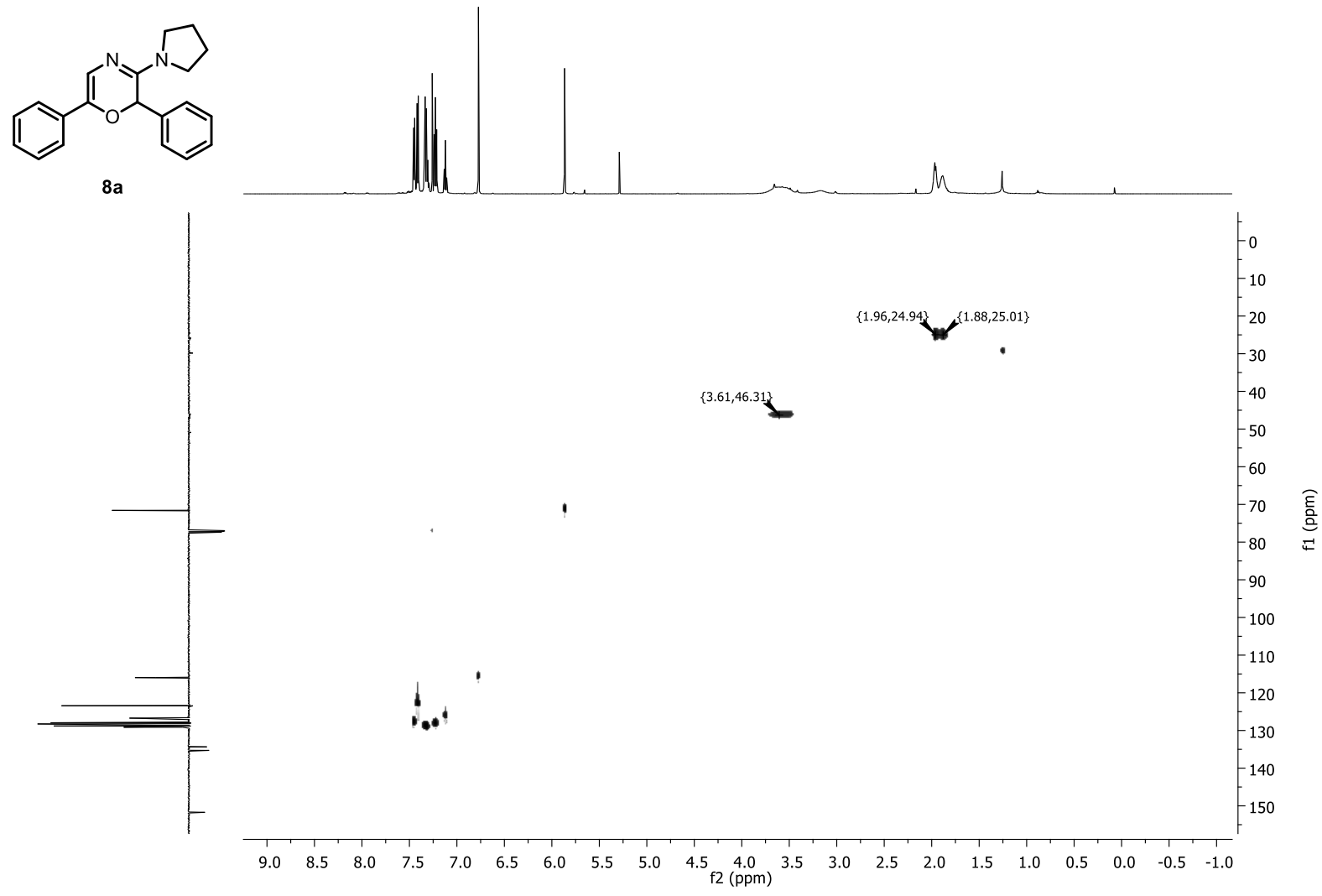

${ }^{1} \mathbf{H}$ NMR $\left(600 \mathrm{MHz}, \mathrm{CDCl}_{3}\right)$

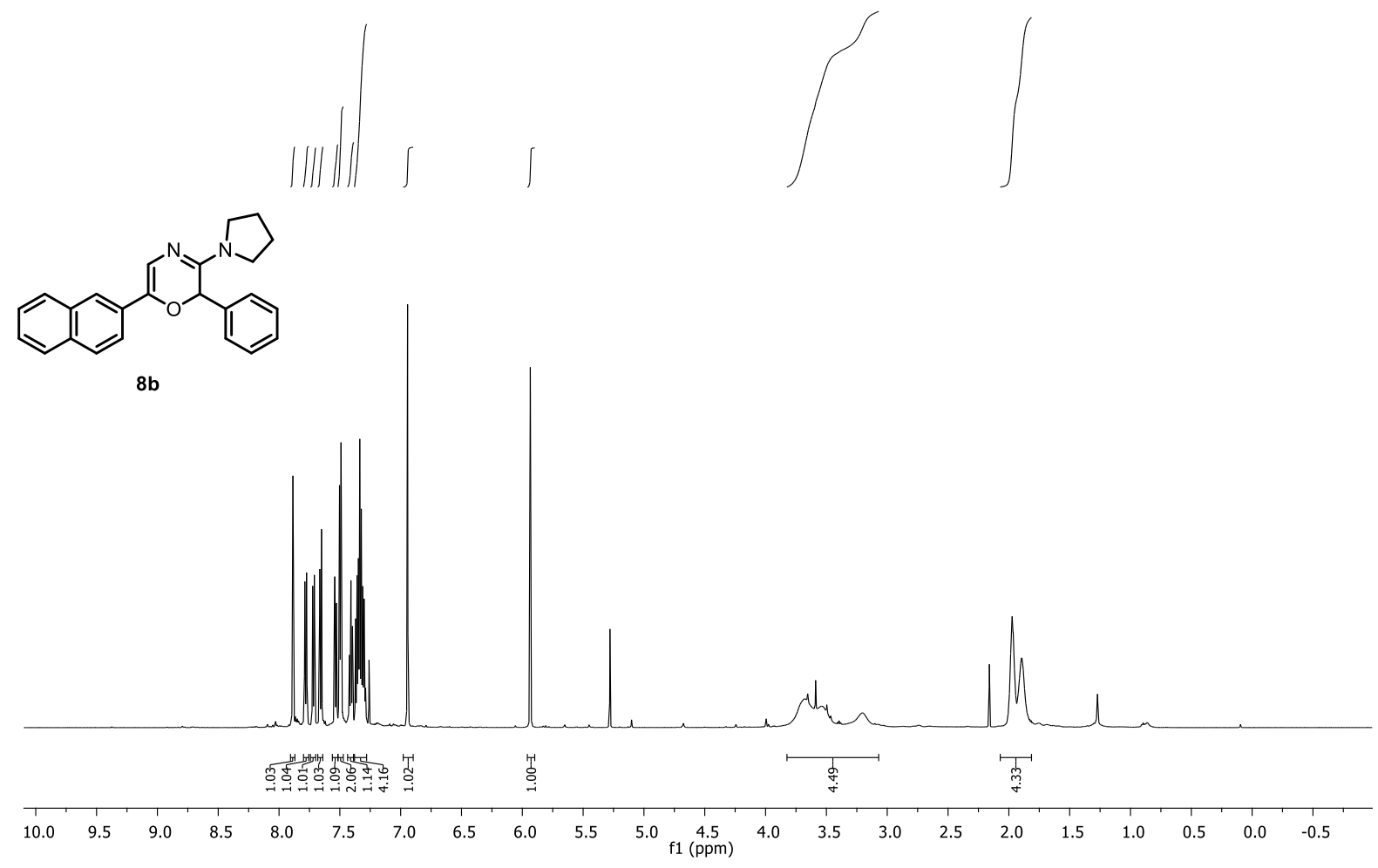

63 
${ }^{13} \mathrm{C}$ NMR (151 MHz, $\left.\mathrm{CDCl}_{3}\right)$
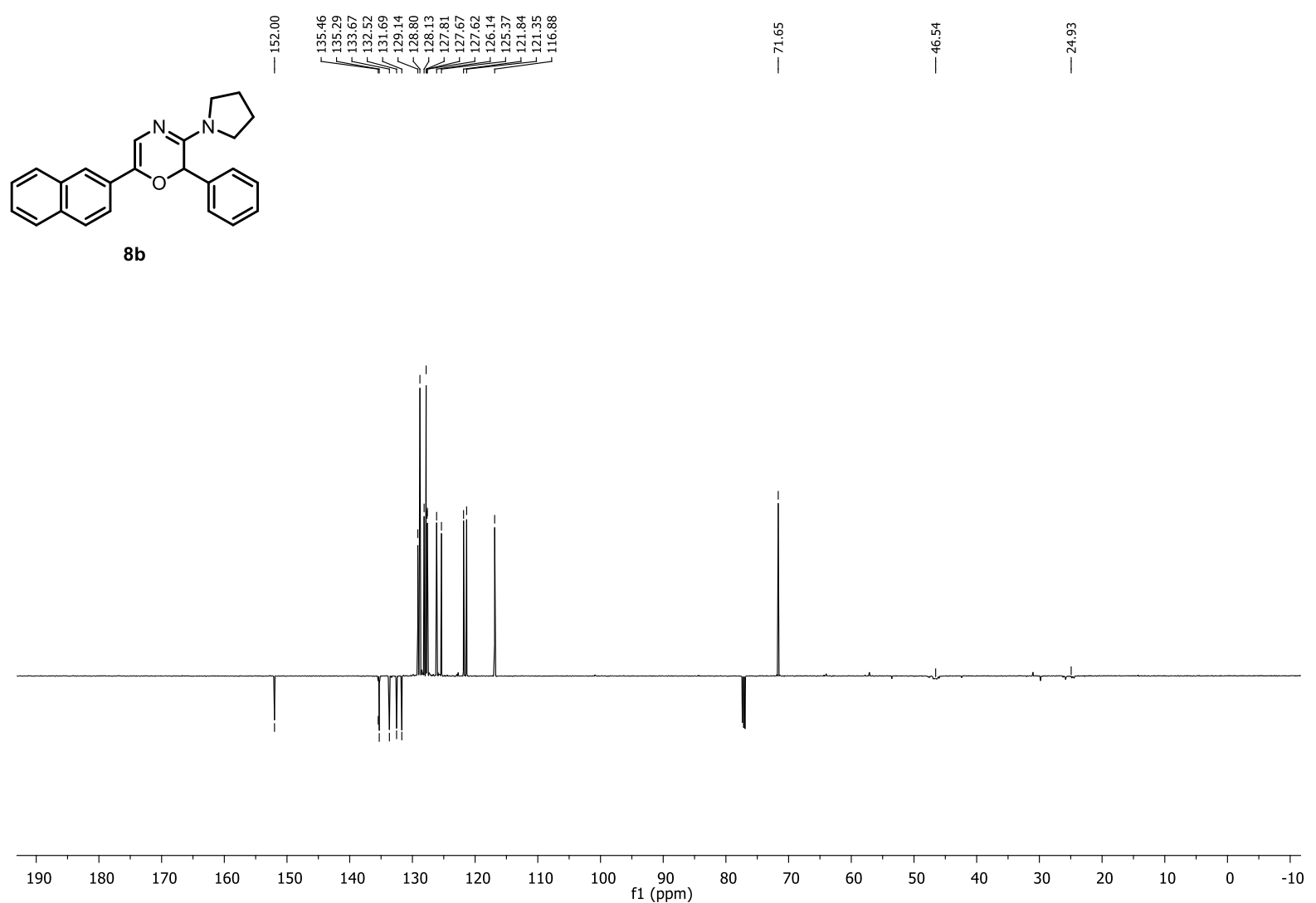

\section{HSQC}

${ }^{1} \mathbf{H}$ NMR $\left(600 \mathrm{MHz}, \mathrm{CDCl}_{3}\right),{ }^{13} \mathrm{C}$ NMR $\left(151 \mathrm{MHz}, \mathrm{CDCl}_{3}\right)$<smiles>C1=C(N2CCCC2)N=C(c2ccc3ccccc3c2)OC1c1ccccc1</smiles>

$8 b$
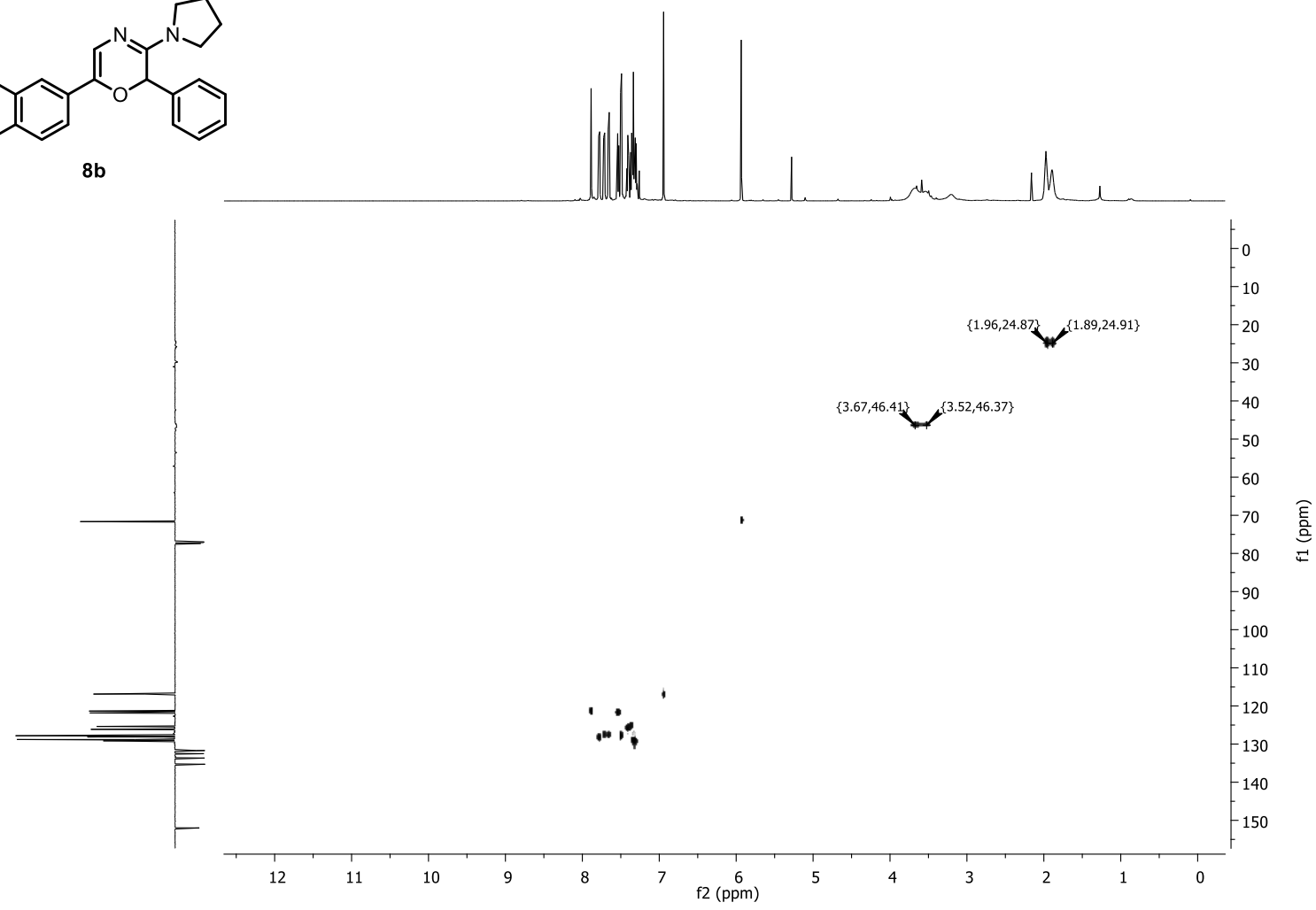
${ }^{1} \mathrm{H}$ NMR $\left(600 \mathrm{MHz}, \mathrm{CDCl}_{3}\right)$

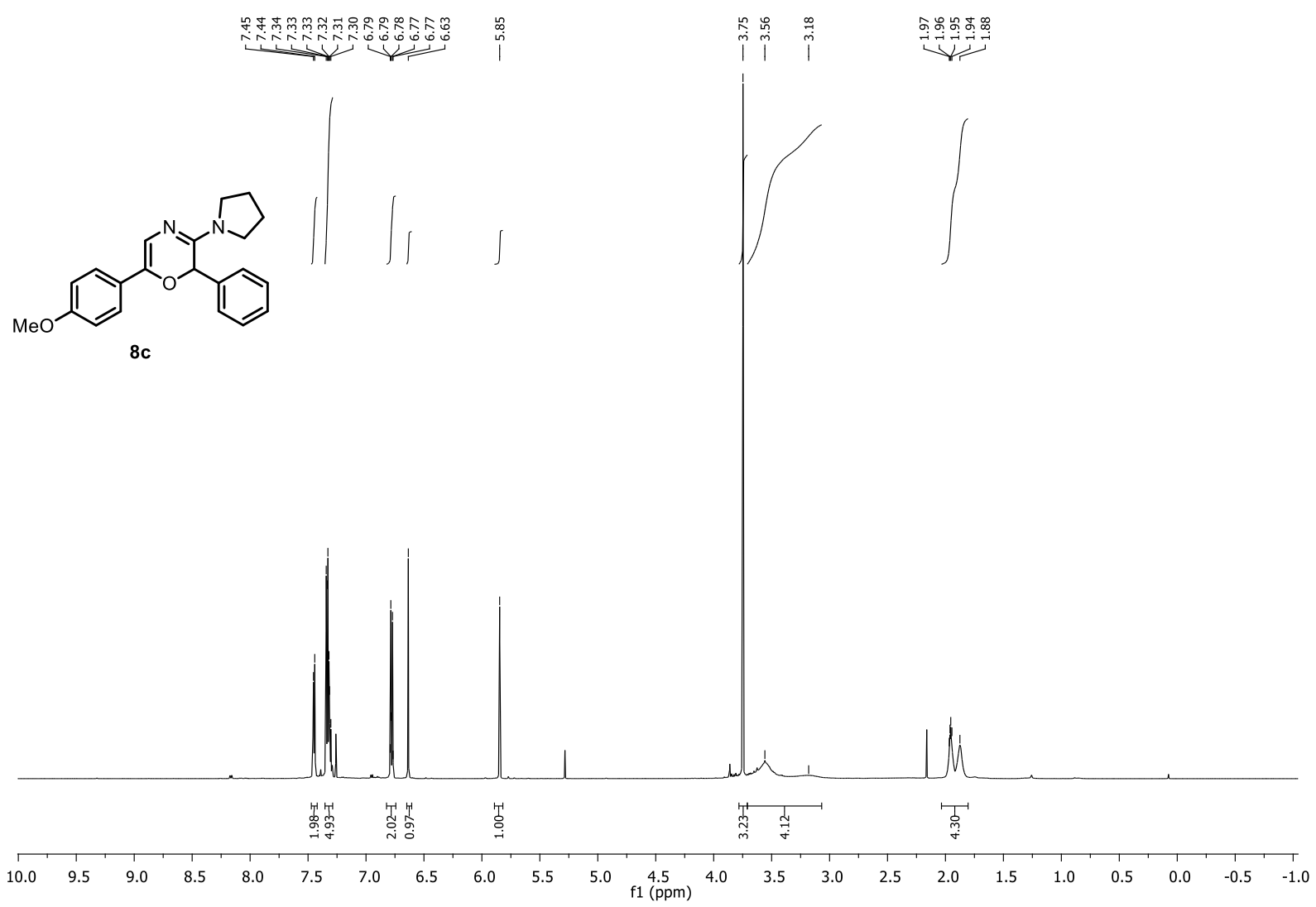

${ }^{13} \mathrm{C}$ NMR $\left(151 \mathrm{MHz}, \mathrm{CDCl}_{3}\right)$

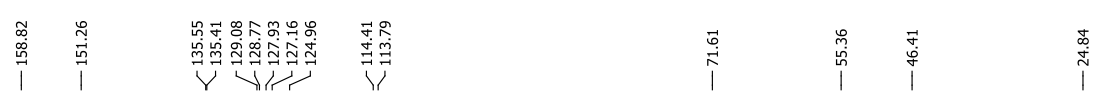

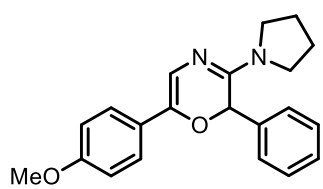

$8 c$

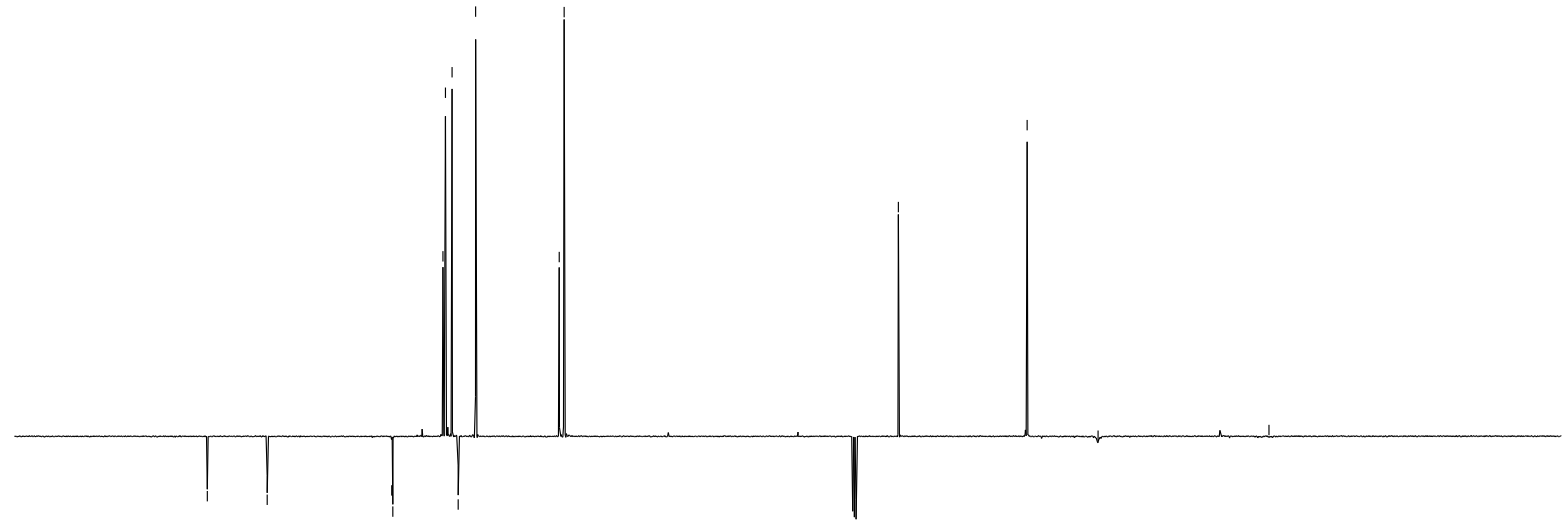

$\begin{array}{lllllllllllllllllll}180 & 170 & 160 & 150 & 140 & 130 & 120 & 110 & 100 & \underset{\mathrm{f} 1(\mathrm{ppm})}{90} \mathbf{8 0} & 70 & 60 & 50 & 40 & 30 & 20 & 10 & 0 & -10\end{array}$ 
HSQC

${ }^{\mathbf{1}} \mathbf{H}$ NMR $\left(600 \mathrm{MHz}, \mathrm{CDCl}_{3}\right),{ }^{13} \mathbf{C}$ NMR (151 MHz, $\left.\mathrm{CDCl}_{3}\right)$

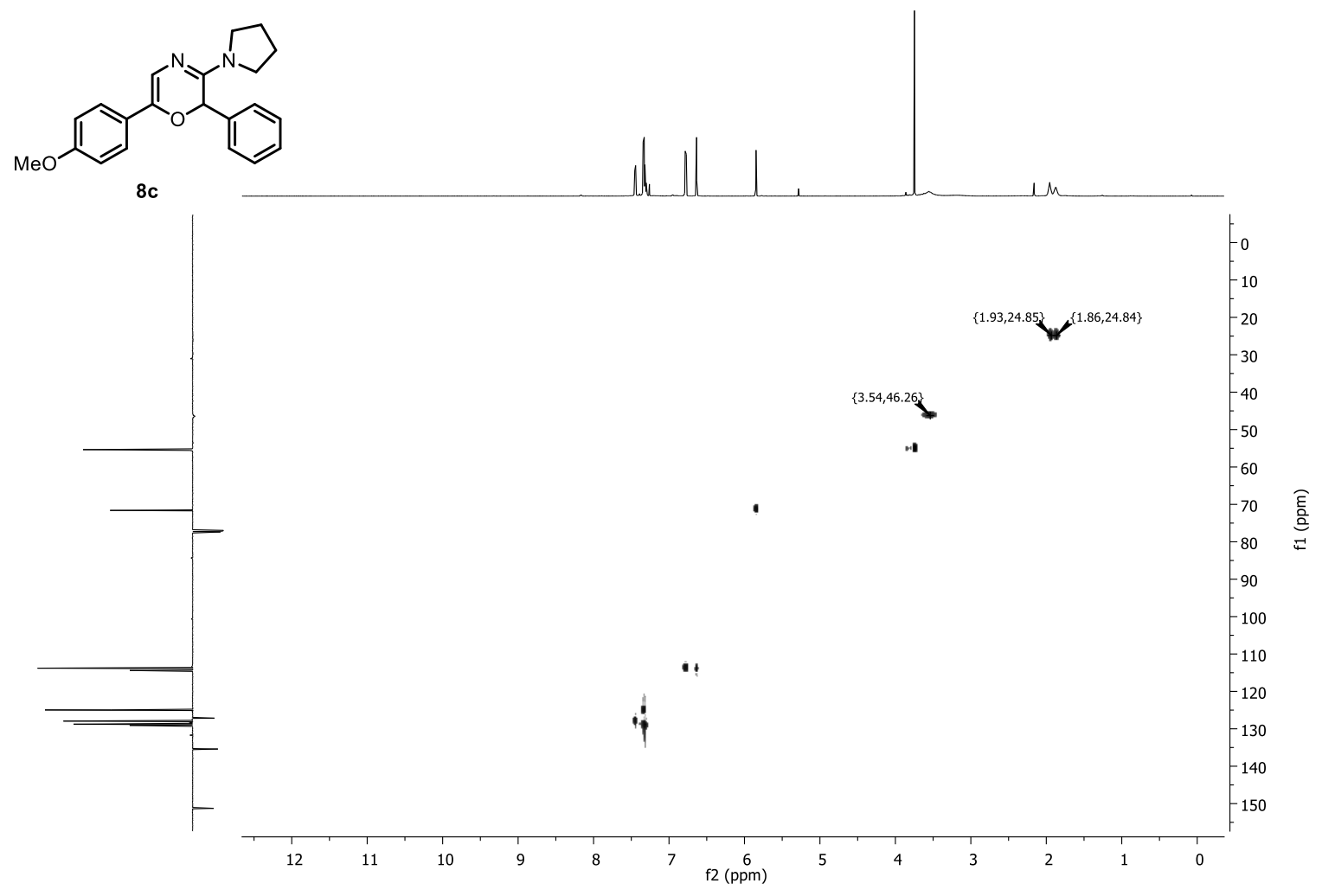

${ }^{1} \mathbf{H} \mathbf{N M R}\left(600 \mathrm{MHz}, \mathrm{CDCl}_{3}\right)$

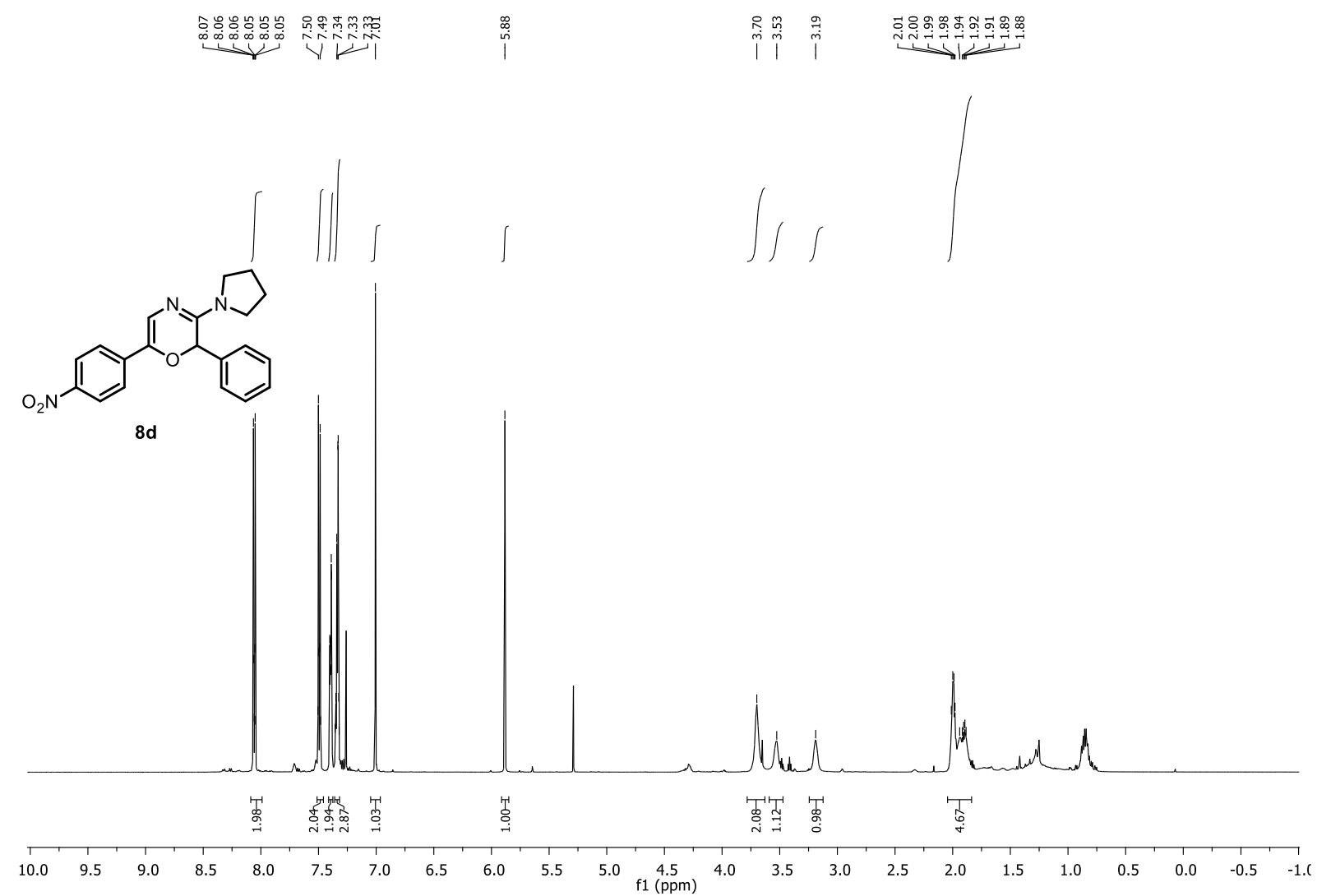


${ }^{13} \mathrm{C}$ NMR (151 MHz, CDCl 3$)$
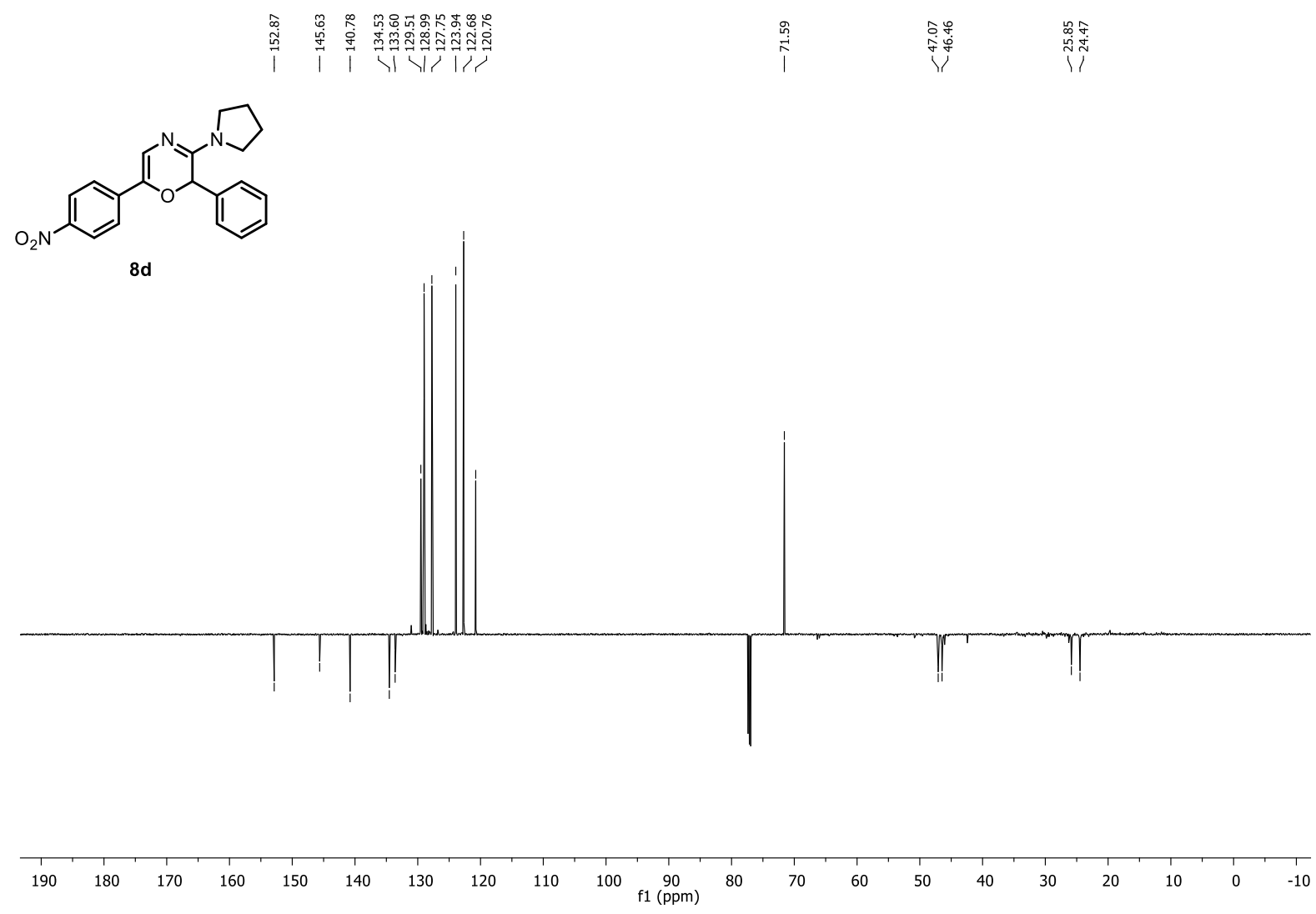

${ }^{1} \mathbf{H}$ NMR (600 MHz, $\left.\mathrm{CDCl}_{3}\right)$

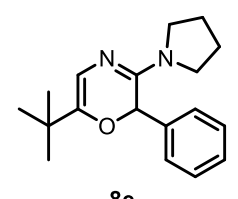

$8 e$
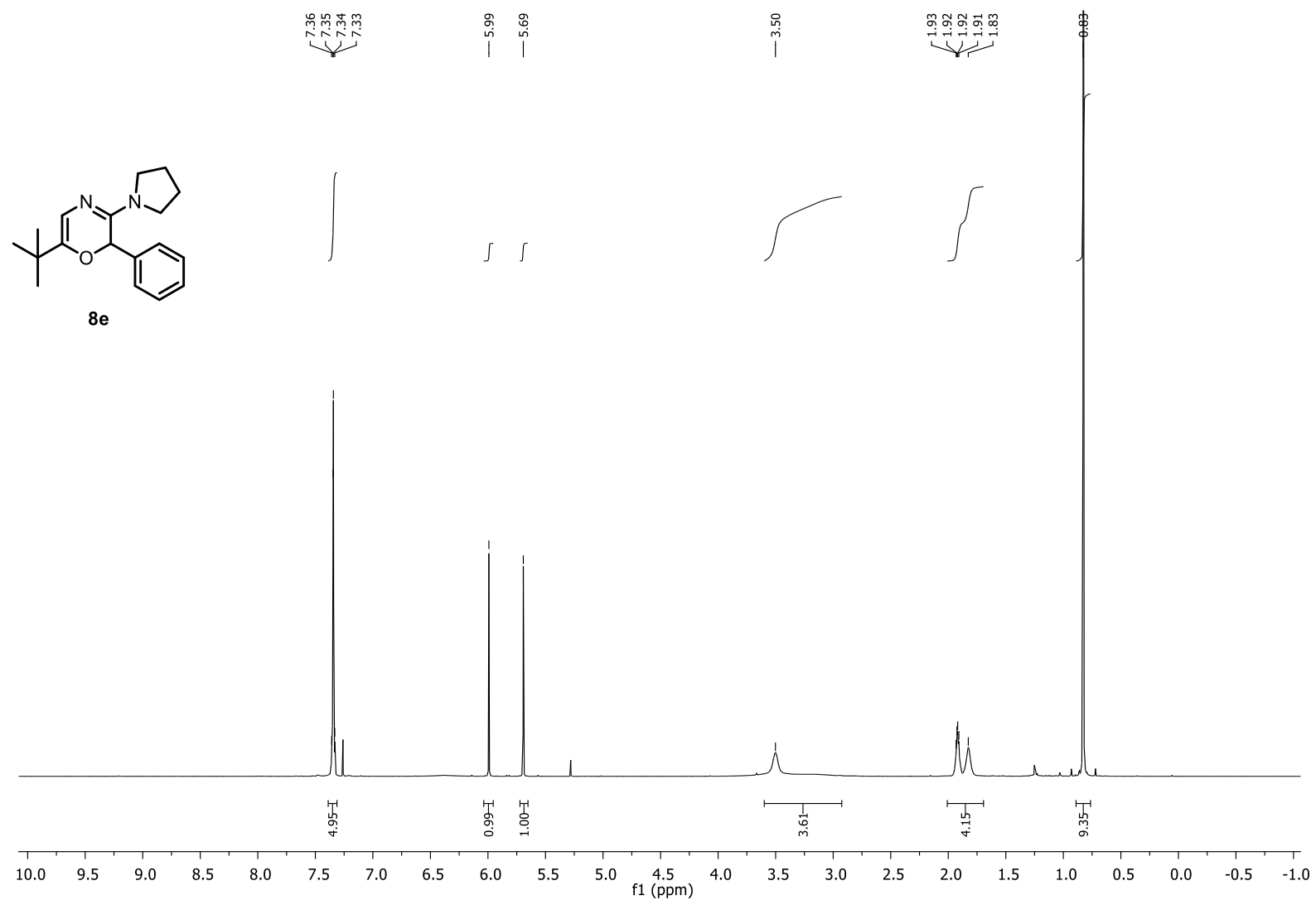

67 
${ }^{13} \mathrm{C}$ NMR (151 MHz, $\left.\mathrm{CDCl}_{3}\right)$
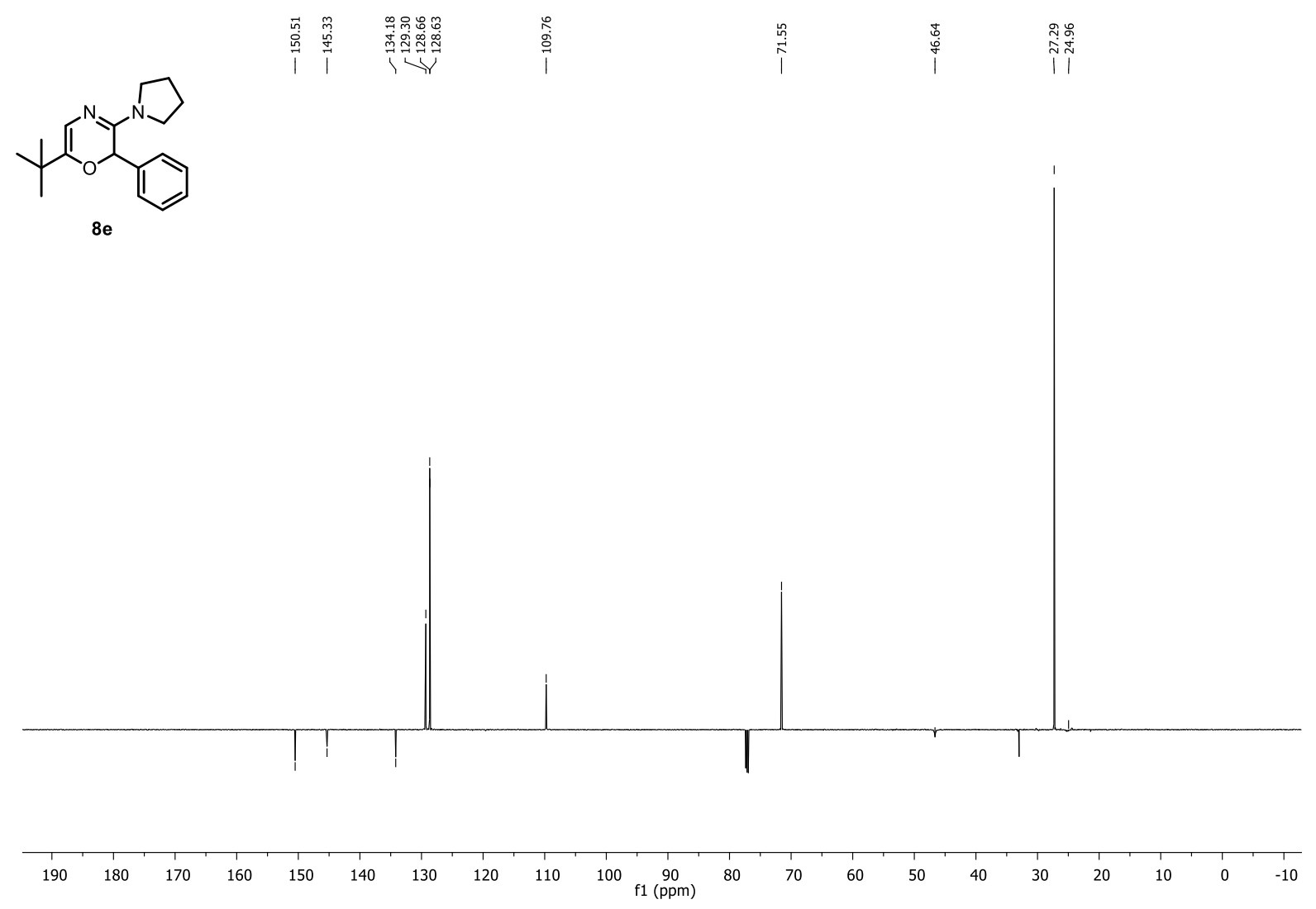

HSQC

${ }^{1} \mathbf{H}$ NMR (600 MHz, CDCl 3$),{ }^{13} \mathrm{C}$ NMR (151 MHz, CDCl 3 )

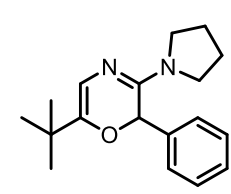

$8 e$

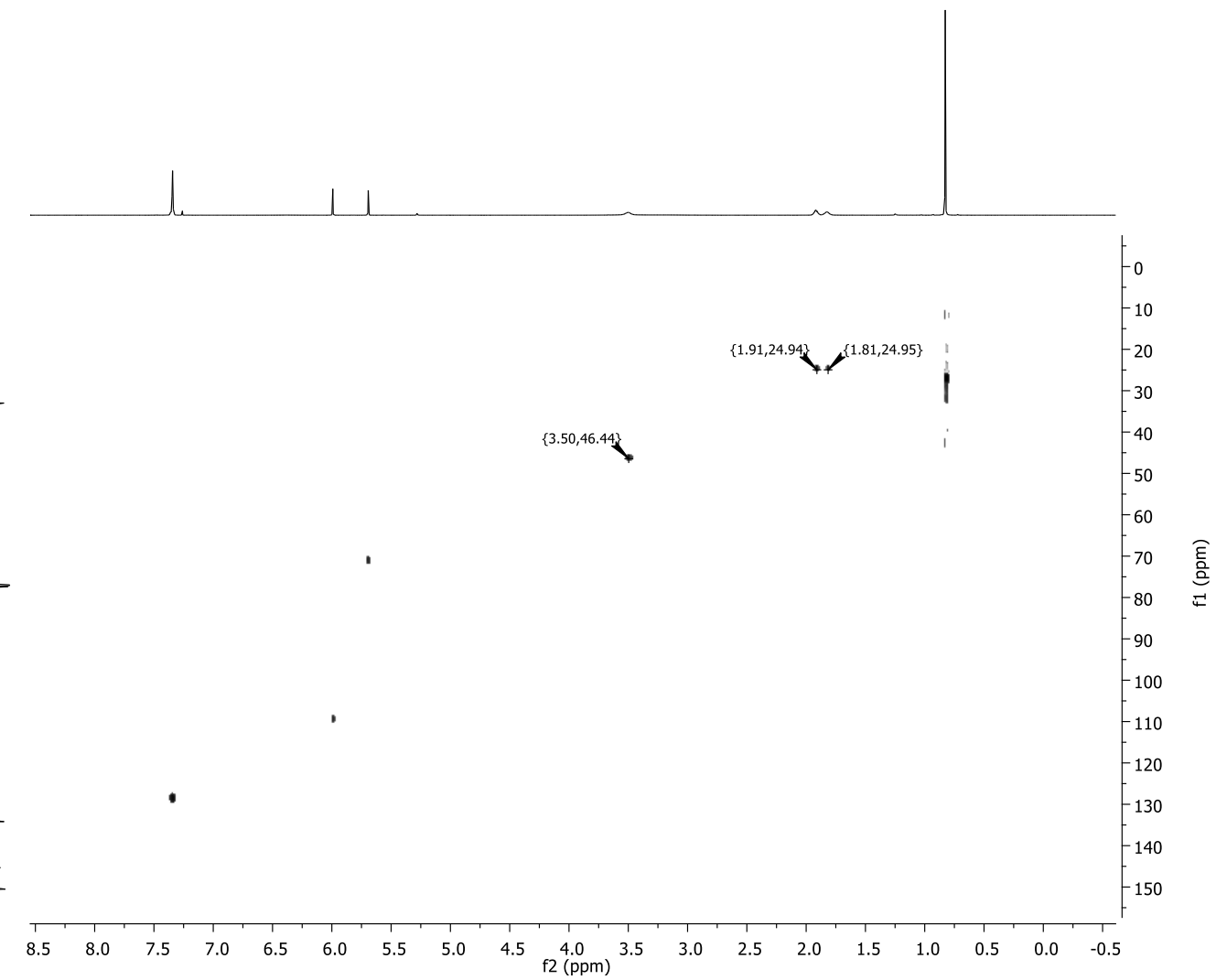

68 
${ }^{1} \mathrm{H}$ NMR $\left(400 \mathrm{MHz}, \mathrm{CDCl}_{3}\right)$

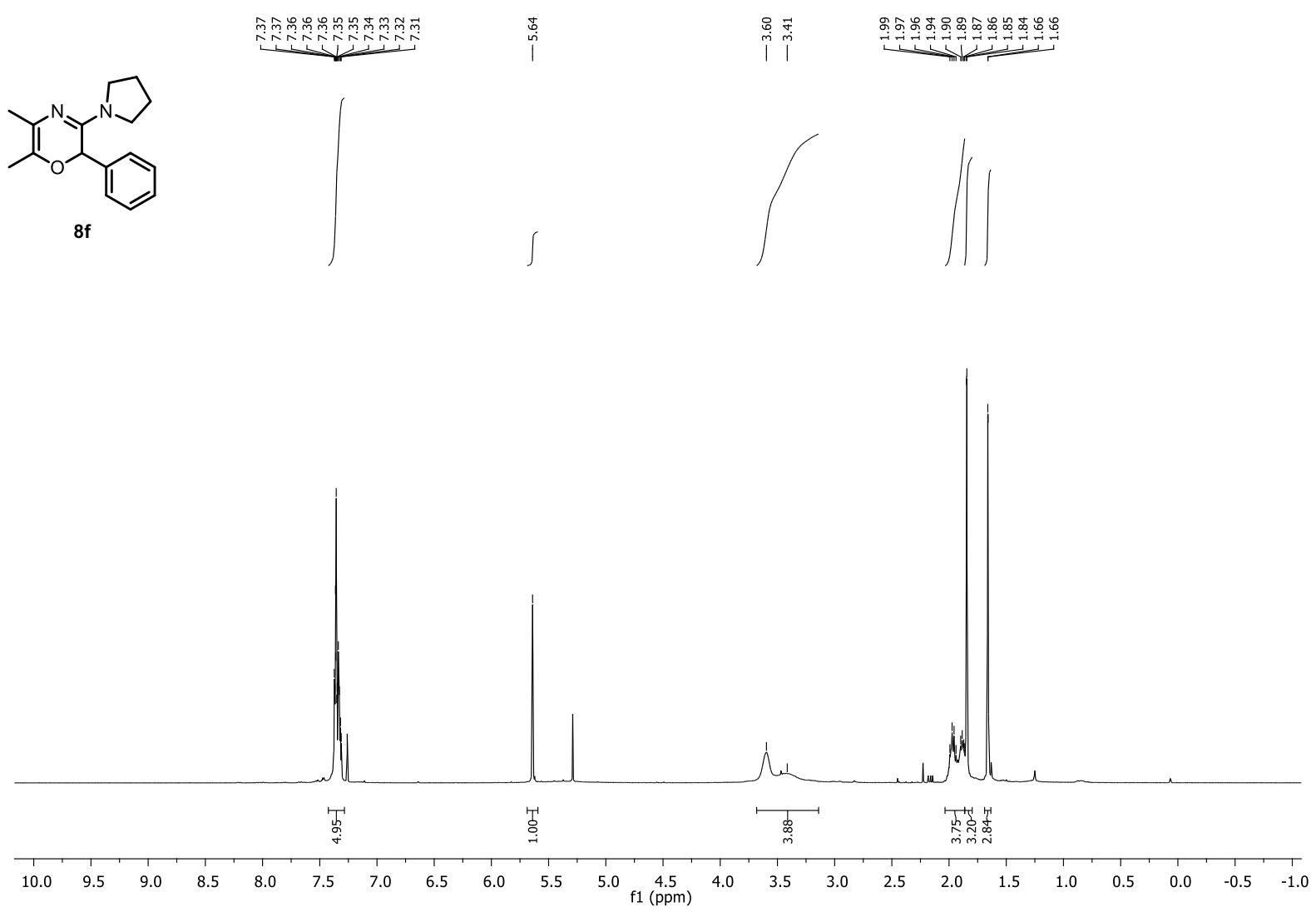

${ }^{13}$ C NMR (151 MHz, DMSO)
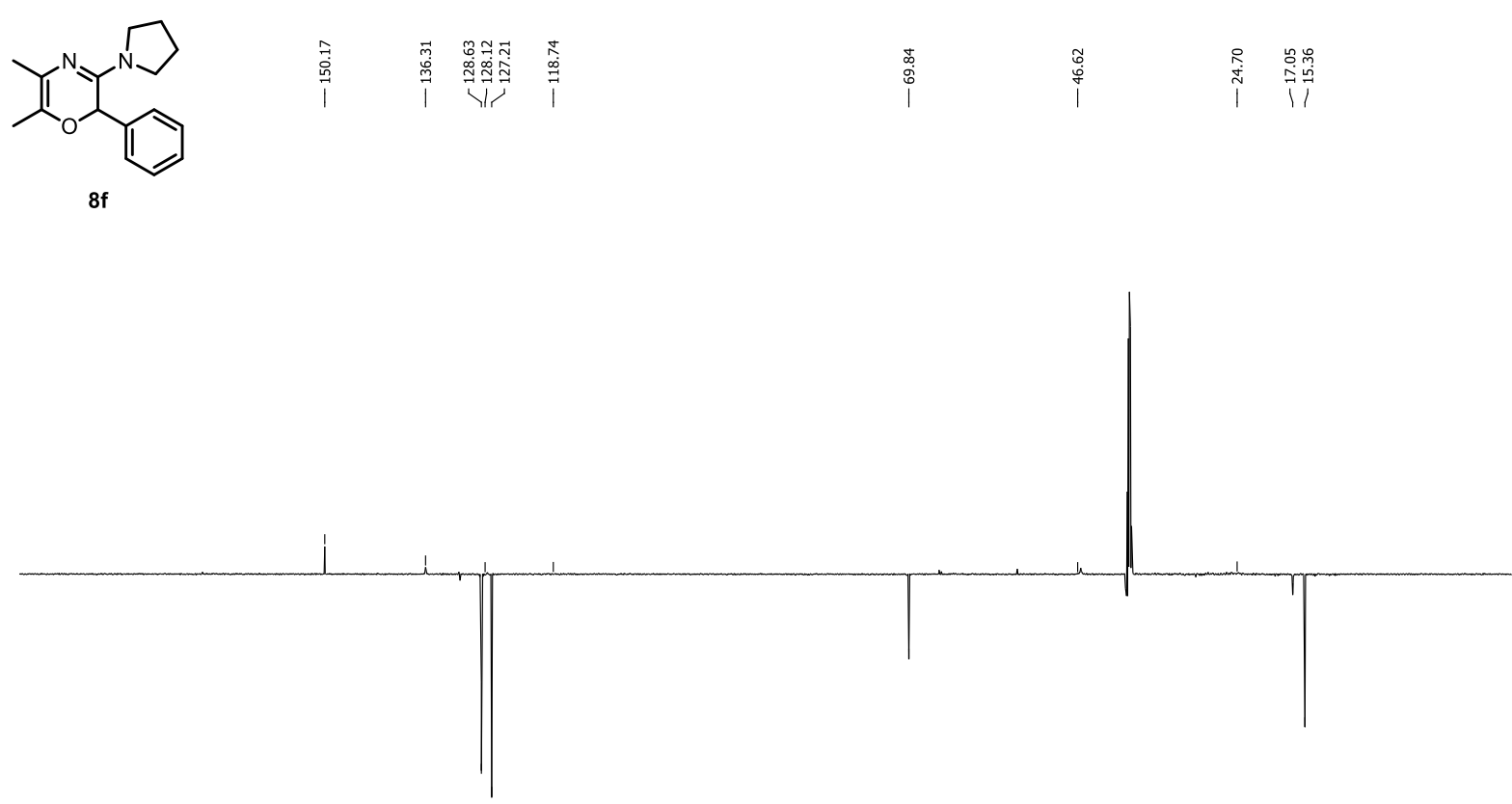

\begin{tabular}{lllllllllllllllllllllll}
\hline 190 & 180 & 170 & 160 & 150 & 140 & 130 & 120 & 110 & 100 & $\underset{\mathrm{f} 1(\mathrm{ppm})}{90}$ & 80 & 70 & 60 & 50 & 40 & 30 & 20 & 10 & 0 & -10
\end{tabular} 
HSQC

${ }^{1} \mathbf{H}$ NMR (600 MHz, CDCl $)$, ${ }^{13} \mathbf{C}$ NMR (151 MHz, DMSO)<smiles>CC1=C(C)OC(c2ccccc2)C(N2CCCC2)=N1</smiles>

$8 f$

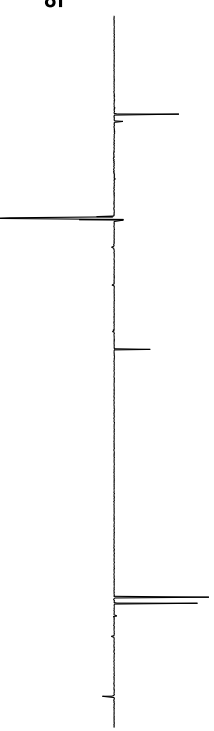

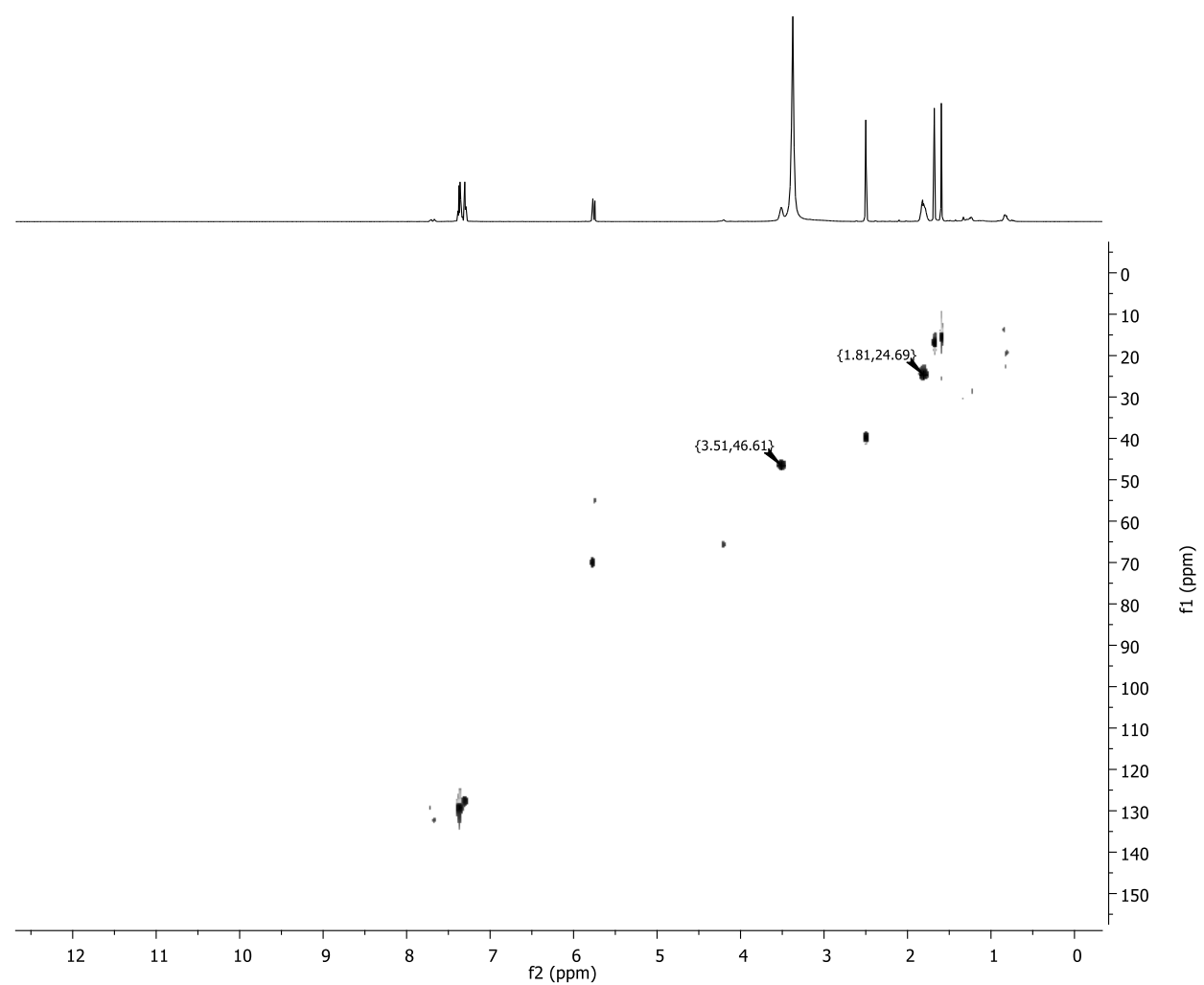

\section{HMBC}

${ }^{1} \mathrm{H}$ NMR $\left(600 \mathrm{MHz}, \mathrm{CDCl}_{3}\right),{ }^{13} \mathrm{C}$ NMR (151 MHz, DMSO)<smiles>Cc1ccnc(-c2nc(C)c(C)o2)n1</smiles>
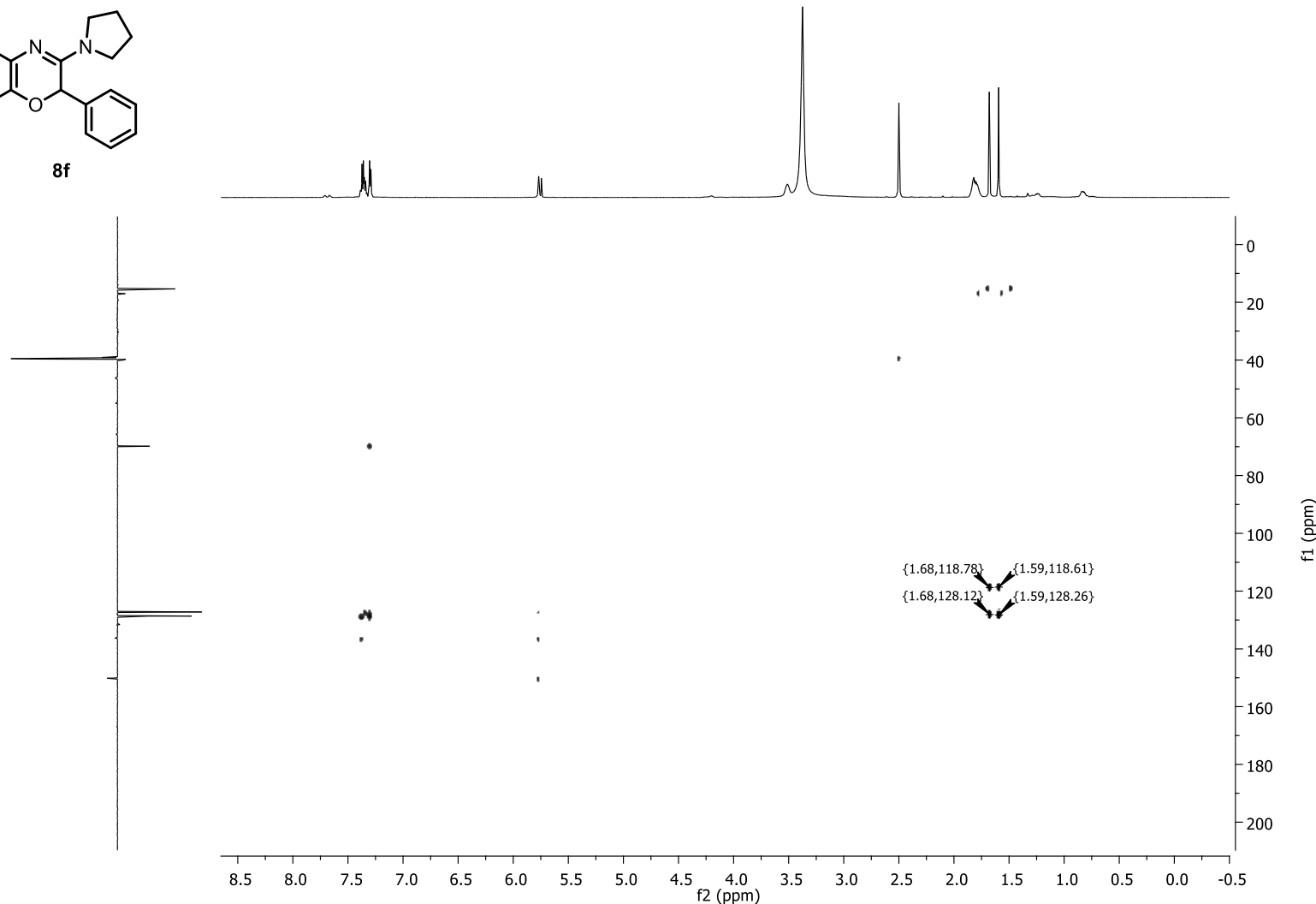
${ }^{1} \mathbf{H}$ NMR $\left(600 \mathrm{MHz}, \mathrm{CDCl}_{3}\right)$

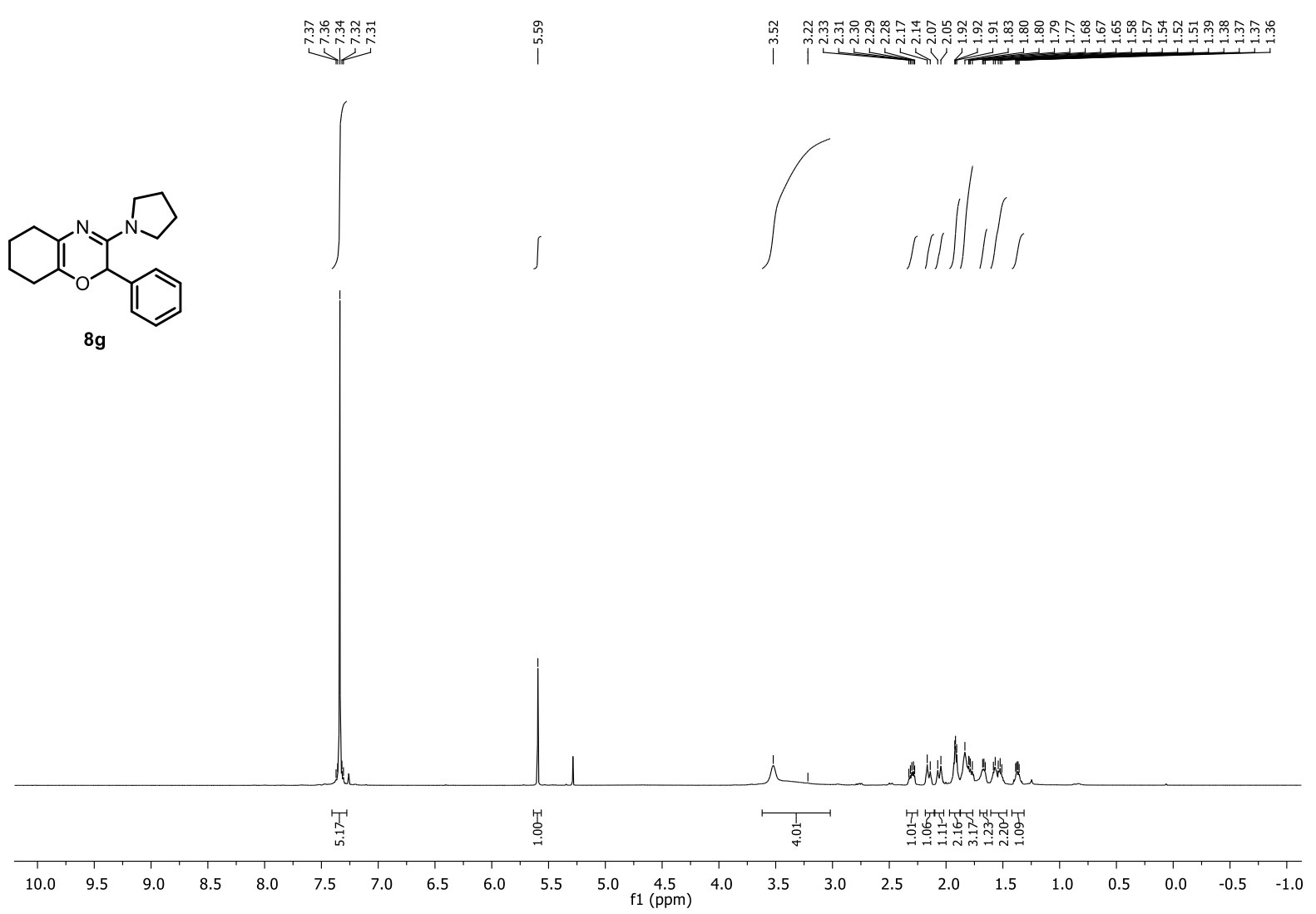

${ }^{13}$ C NMR (151 MHz, $\left.\mathrm{CDCl}_{3}\right)$<smiles>c1ccc(C2OC3=C(CCCC3)N=C2N2CCCC2)cc1</smiles>

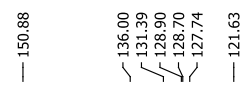

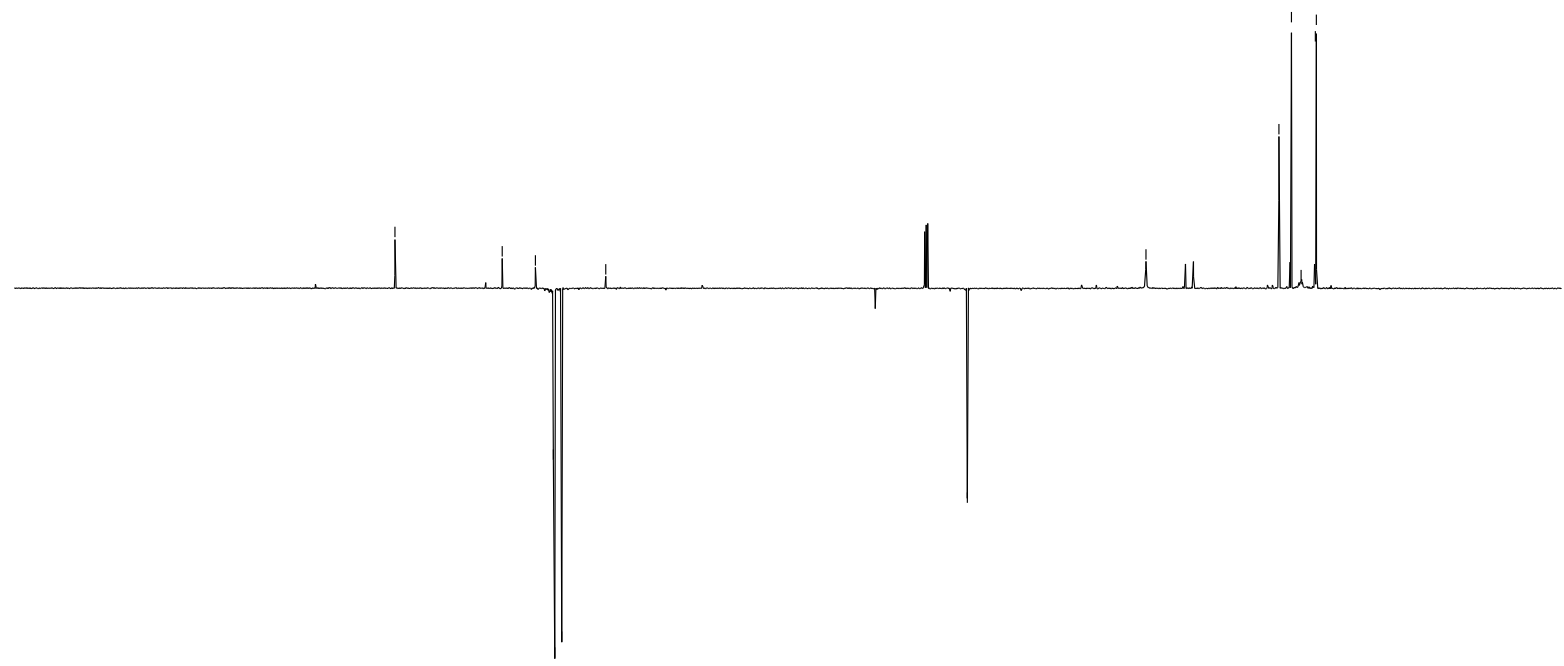

$\begin{array}{lllll}160 & 150 & 140 & 130\end{array}$

$\begin{array}{lll}110 & 100 & 90\end{array}$

80

60

40

$\begin{array}{lllll}30 & 20 & 10 & 0 & -10\end{array}$ 
${ }^{1} \mathbf{H}$ NMR (600 MHz, $\left.\mathrm{CDCl}_{3}\right)$

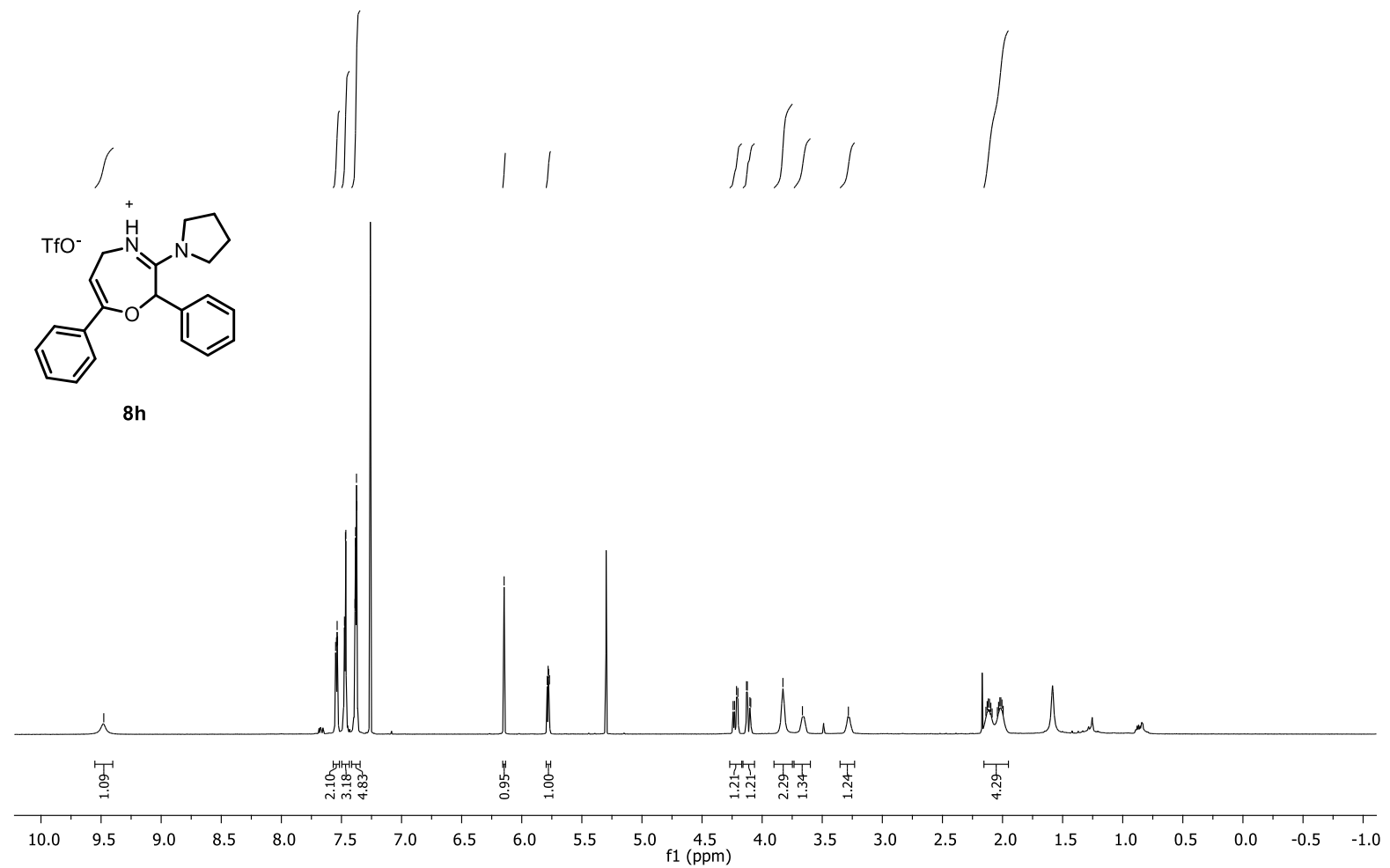

${ }^{13} \mathrm{C}$ NMR (151 MHz, $\left.\mathrm{CDCl}_{3}\right)$

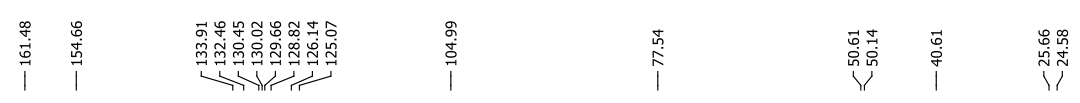<smiles>C1=C(c2ccccc2)OC(c2ccccc2)C(N2CCCC2)=NC1</smiles>

$8 h$

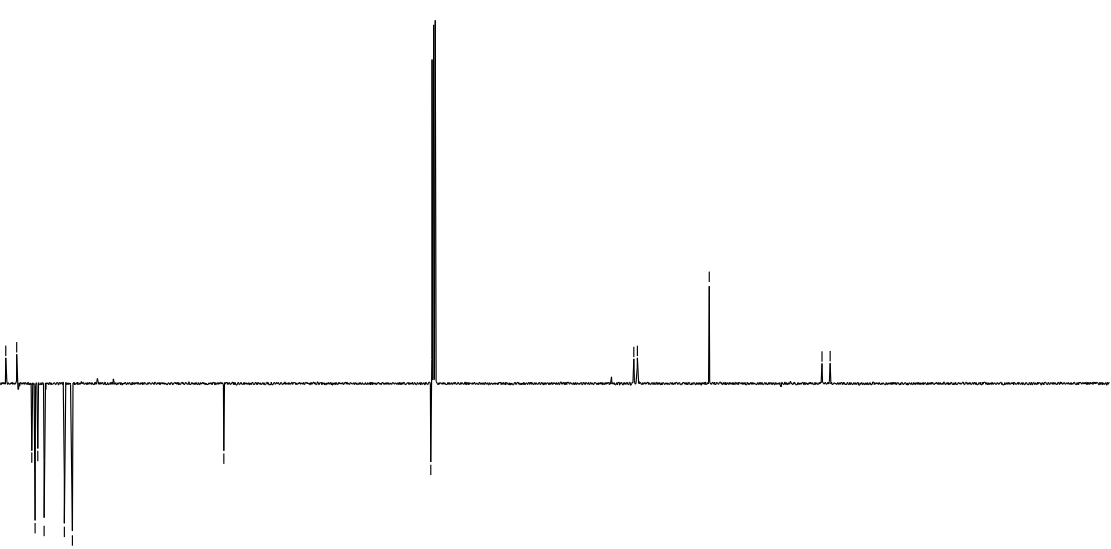

$\begin{array}{lllllllllllllllllllllllllllllllll}190 & 180 & 170 & 160 & 150 & 140 & 130 & 120 & 110 & 100 & 90 & 80 & 70 & 60 & 50 & 40 & 30 & 20 & 10 & 0 & -10\end{array}$ 
${ }^{19}$ F NMR (565 MHz, $\mathrm{CDCl}_{3}$ )

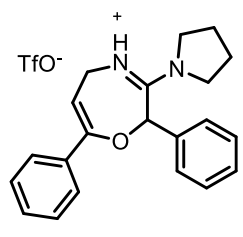

$8 h$

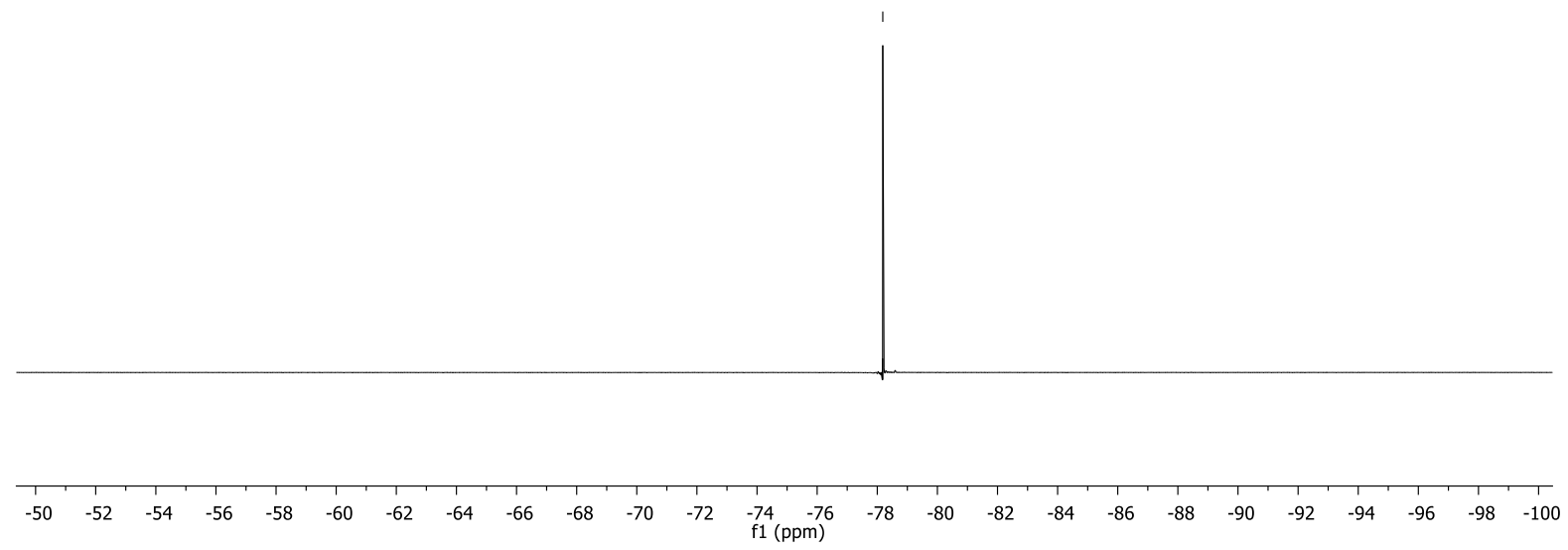

${ }^{1} \mathbf{H}$ NMR (600 MHz, $\mathrm{CDCl}_{3}$ )<smiles>O=C1CN=C(N2CCCC2)C(c2ccccc2)O1</smiles>

8i

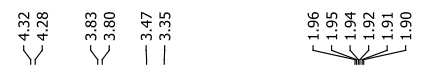

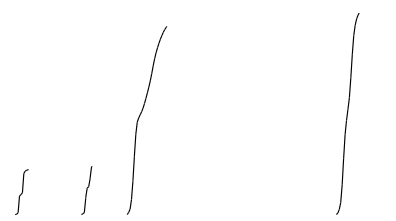

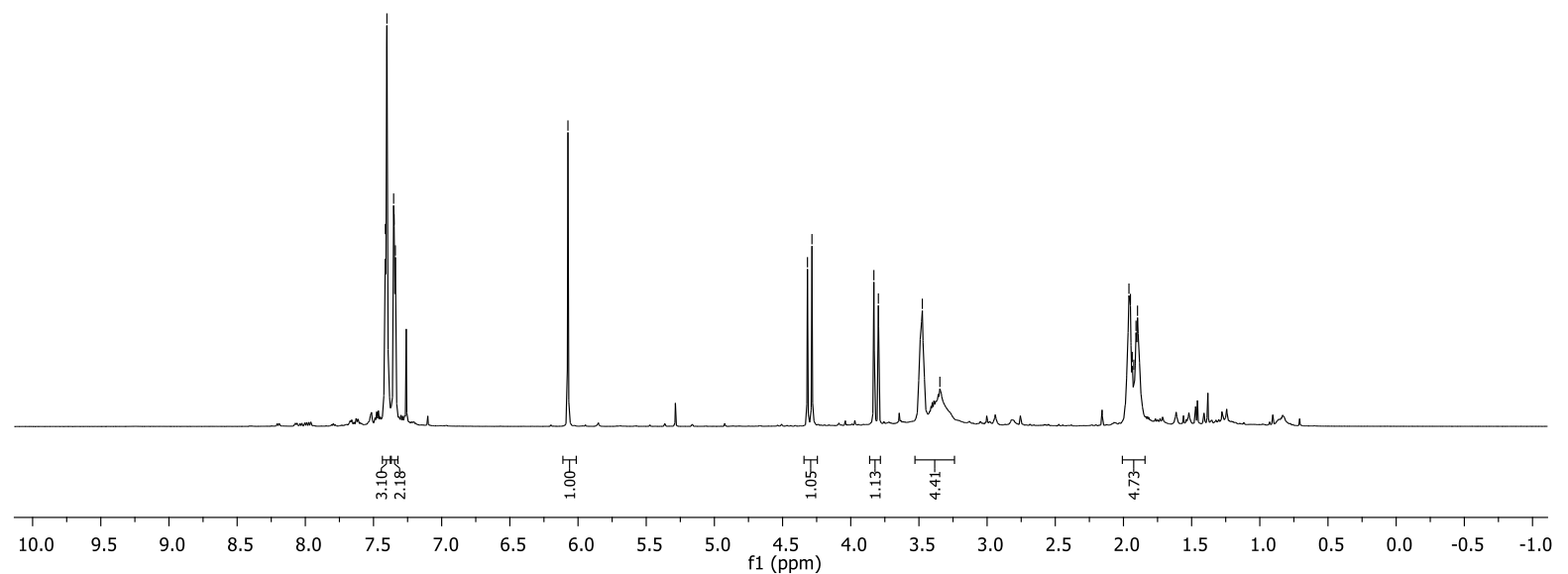


${ }^{13} \mathrm{C}$ NMR $\left(151 \mathrm{MHz}, \mathrm{CDCl}_{3}\right)$

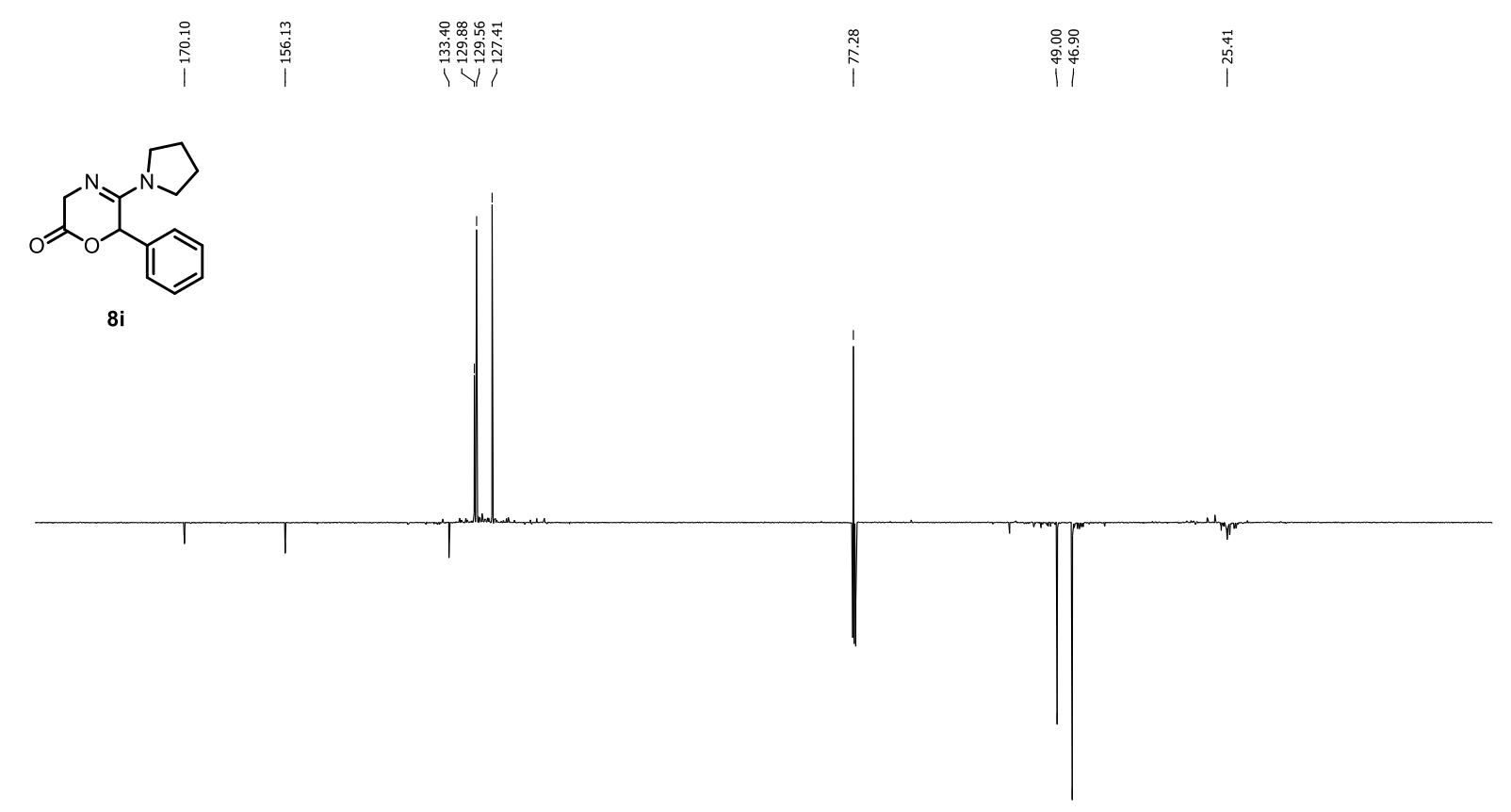

\begin{tabular}{rllllllllllllllllllllll}
\hline 190 & 180 & 170 & 160 & 150 & 140 & 130 & 120 & 110 & 100 & $\begin{array}{c}90 \\
\mathrm{f} 1(\mathrm{ppm})\end{array}$ & 80 & 70 & 60 & 50 & 40 & 30 & 20 & 10 & 0 & -10
\end{tabular}

${ }^{1} \mathbf{H}$ NMR $\left(600 \mathrm{MHz}, \mathrm{CDCl}_{3}\right)$

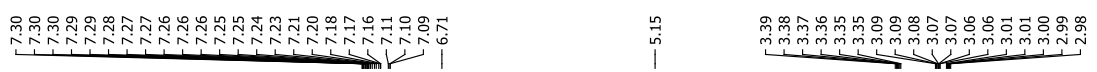<smiles>O=C1NCCc2ccccc2C1c1ccccc1</smiles>

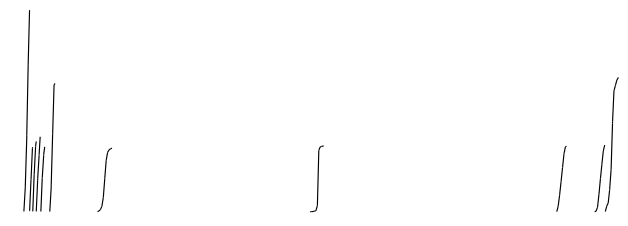

$4 a$

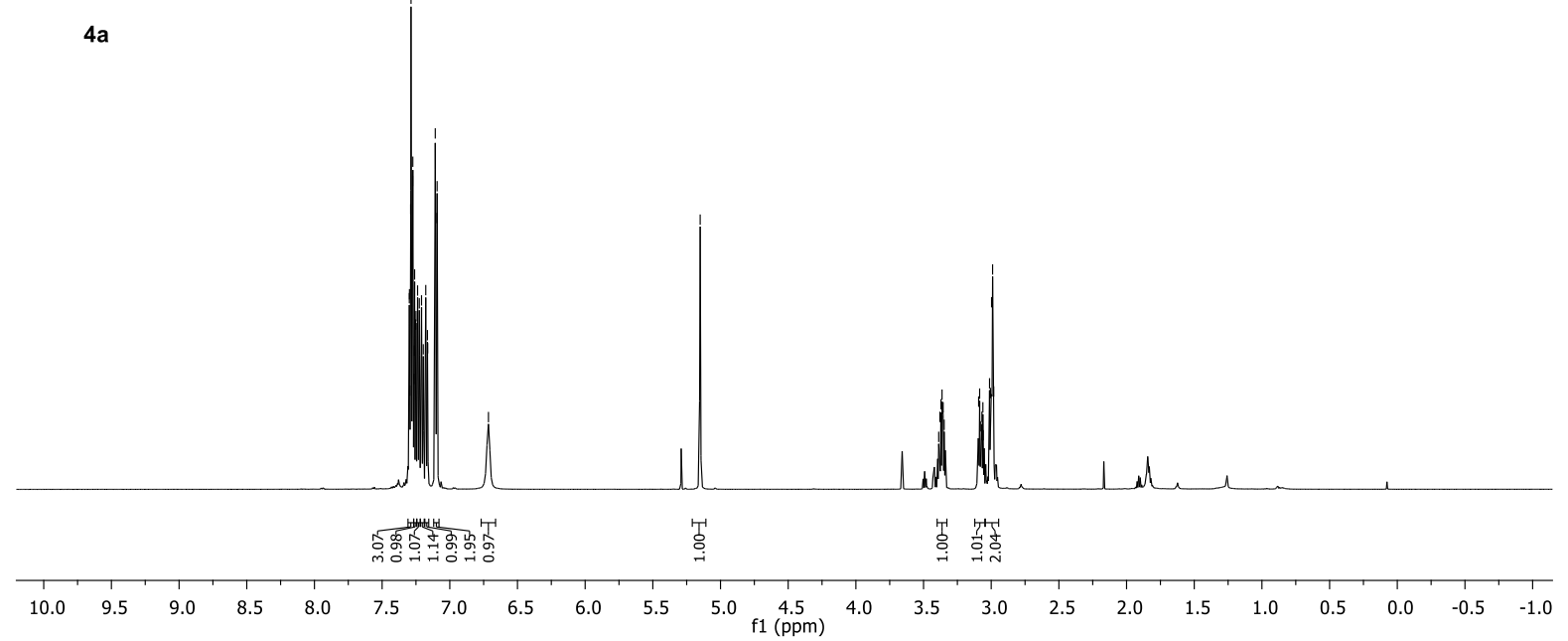


${ }^{13} \mathrm{C}$ NMR $\left(151 \mathrm{MHz}, \mathrm{CDCl}_{3}\right)$

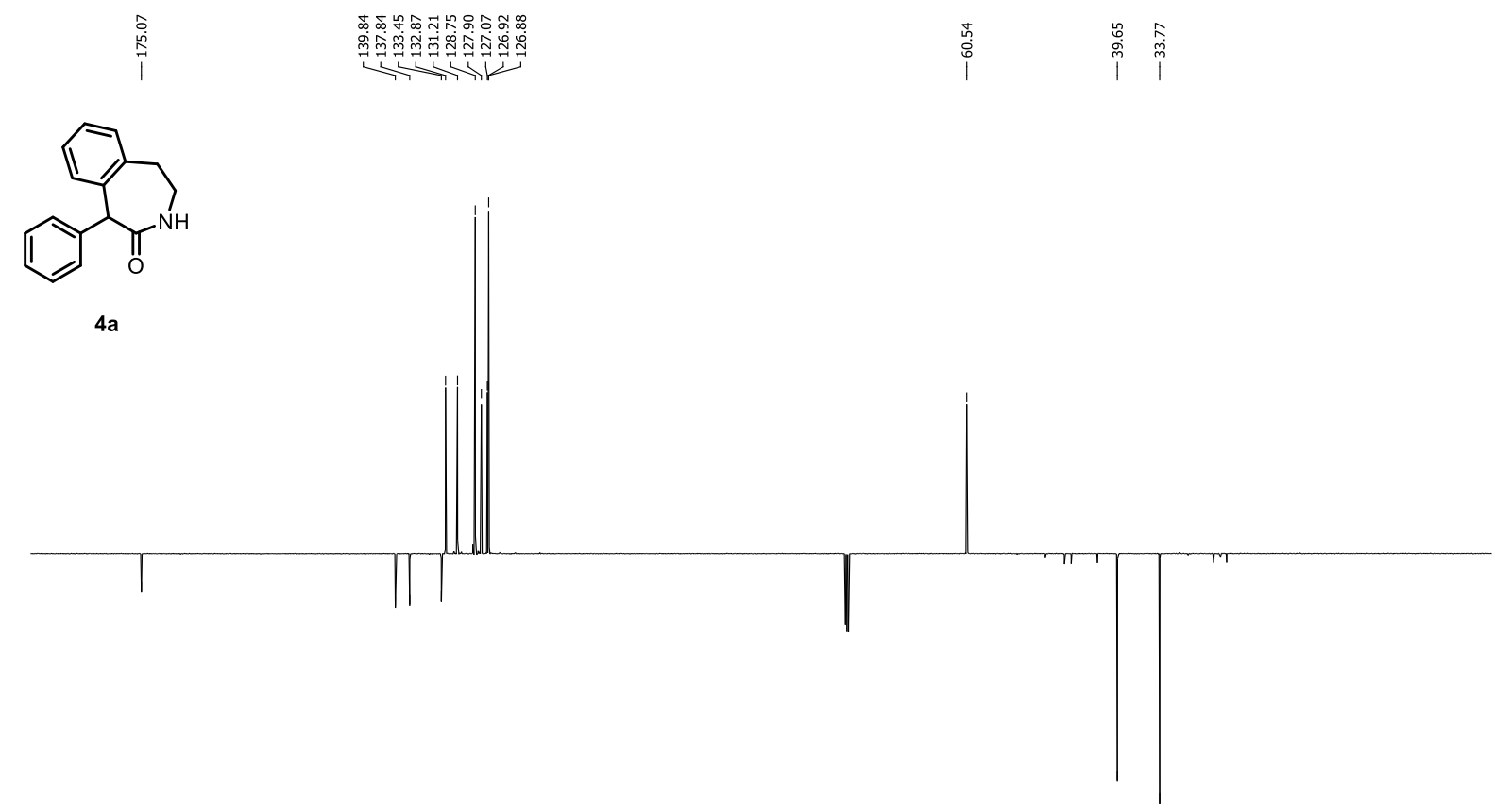

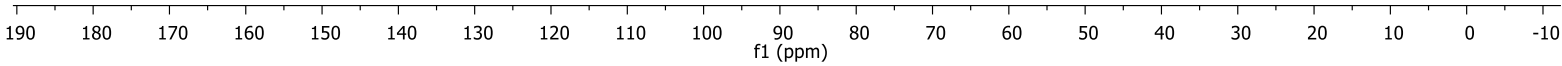

${ }^{1} \mathbf{H}$ NMR $\left(600 \mathrm{MHz}, \mathrm{CDCl}_{3}\right)$

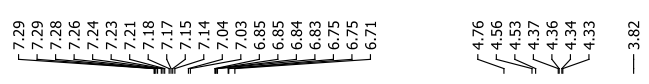<smiles>COc1ccc2c(c1)CNC(=O)C2c1ccccc1</smiles>

$\|||||$

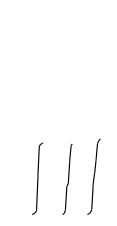

$4 \mathrm{ba}$

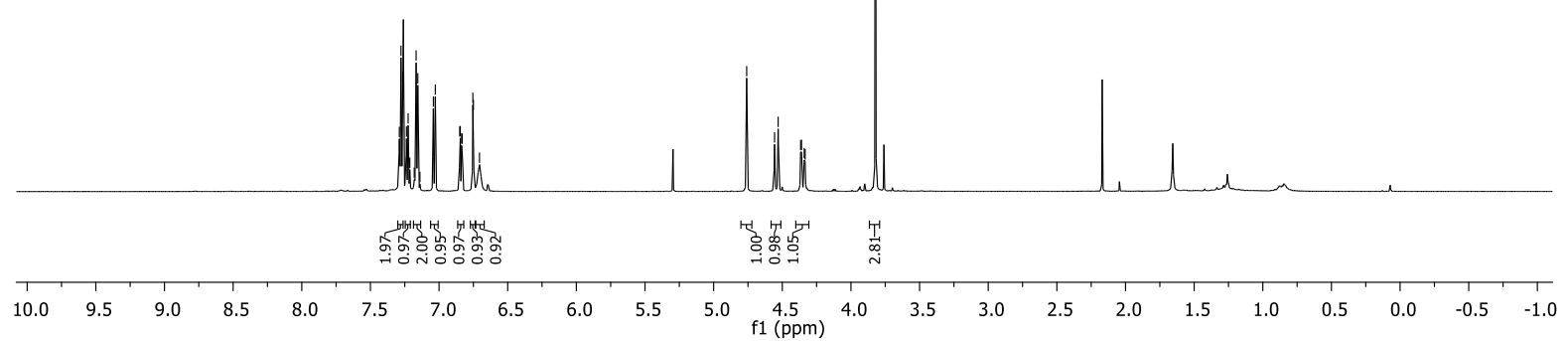


${ }^{13}$ C NMR (151 MHz, $\left.\mathrm{CDCl}_{3}\right)$
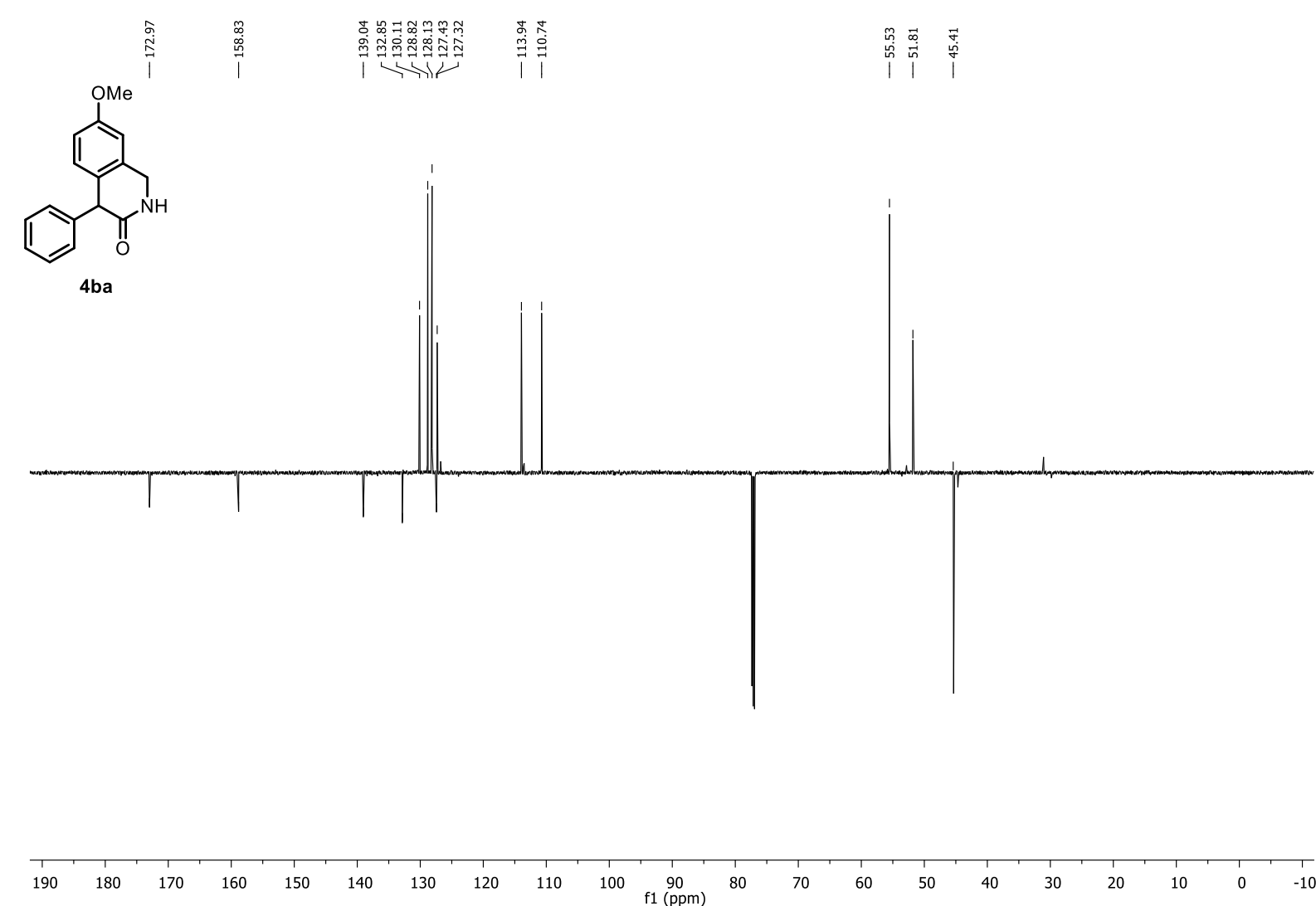

${ }^{1} \mathrm{H}$ NMR $\left(600 \mathrm{MHz}, \mathrm{CDCl}_{3}\right)$

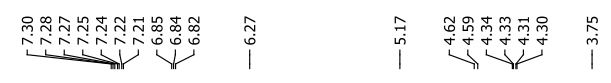<smiles>COc1ccc2c(c1)C(c1ccccc1)C(=O)NC2</smiles>

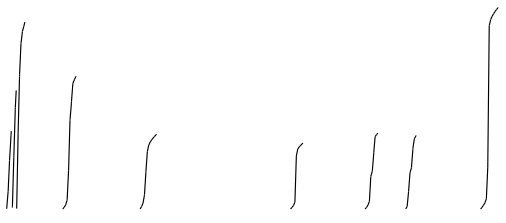

$4 b b$

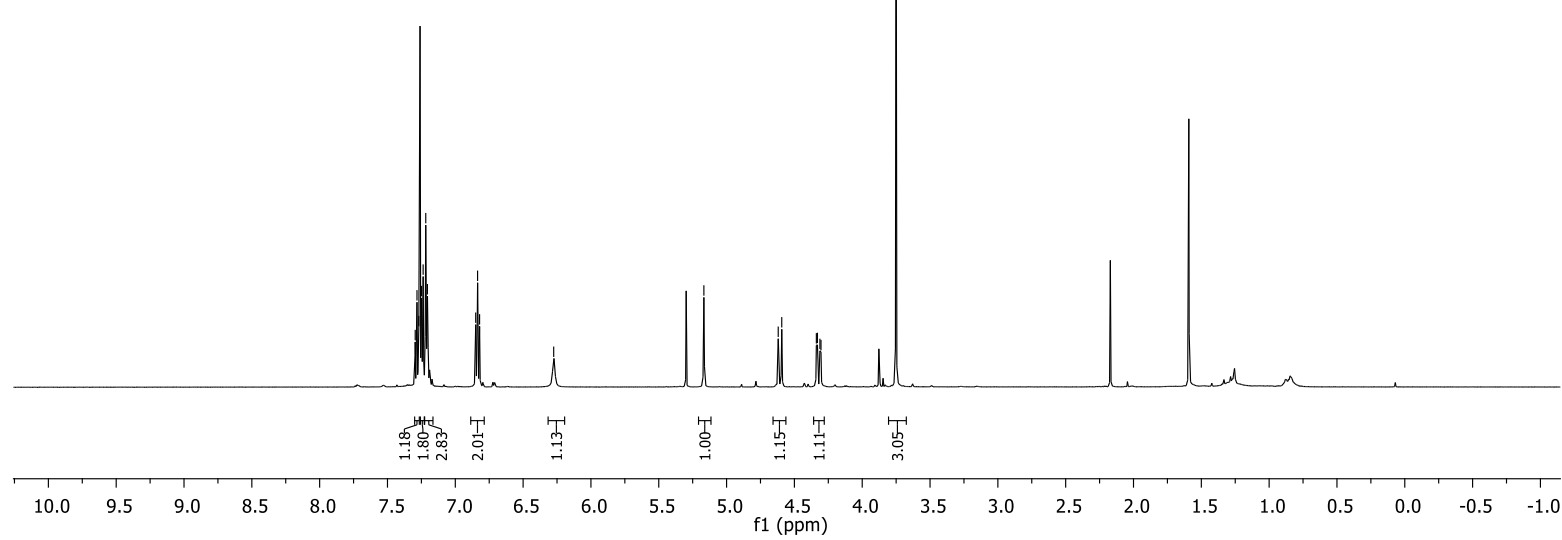


${ }^{13} \mathrm{C}$ NMR $\left(151 \mathrm{MHz}, \mathrm{CDCl}_{3}\right)$

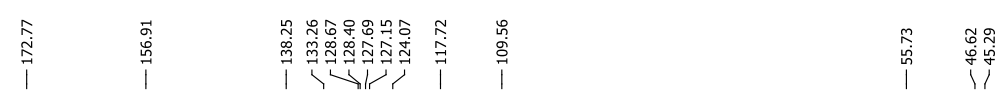<smiles>COc1ccc2c(c1)C(c1ccccc1)C(=O)NC2</smiles>

$4 \mathrm{bb}$

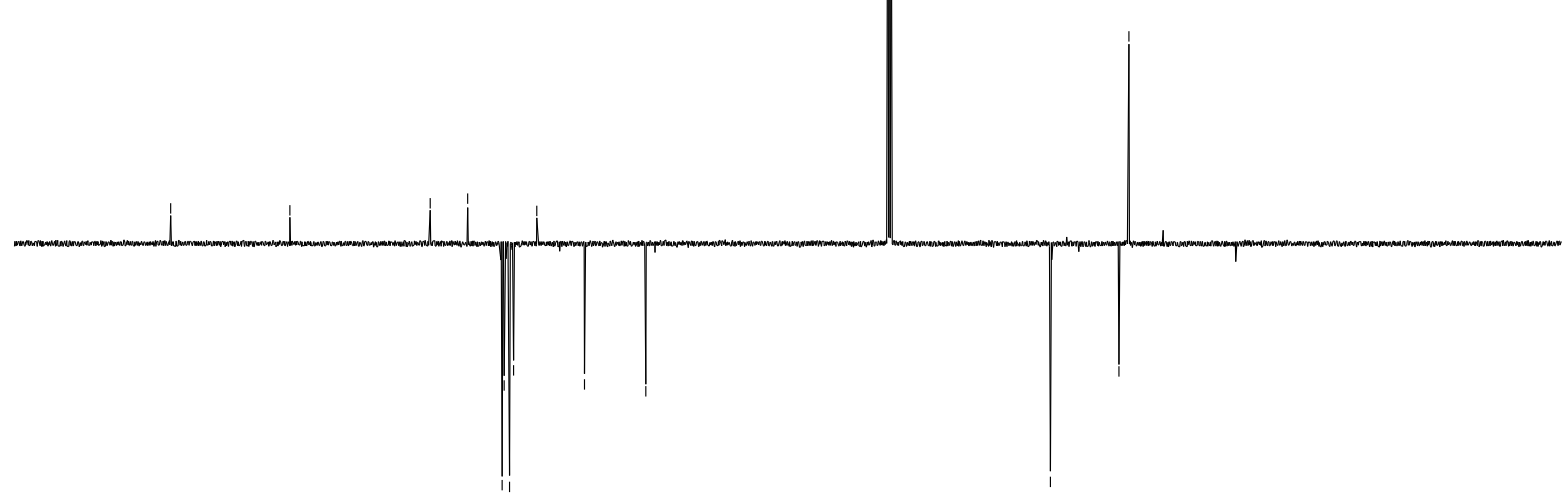

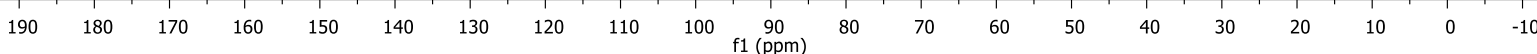

${ }^{1} \mathrm{H}$ NMR $\left(600 \mathrm{MHz}, \mathrm{CDCl}_{3}\right)$

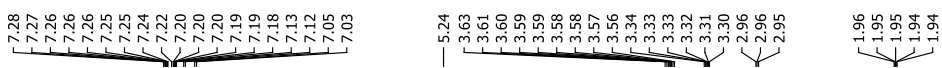<smiles>c1ccc(C2C(N3CCCC3)=NCCc3ccccc32)cc1</smiles>
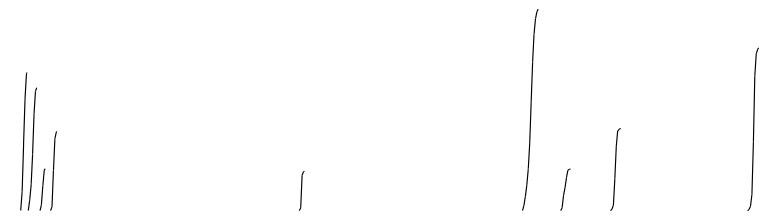

$5 a$

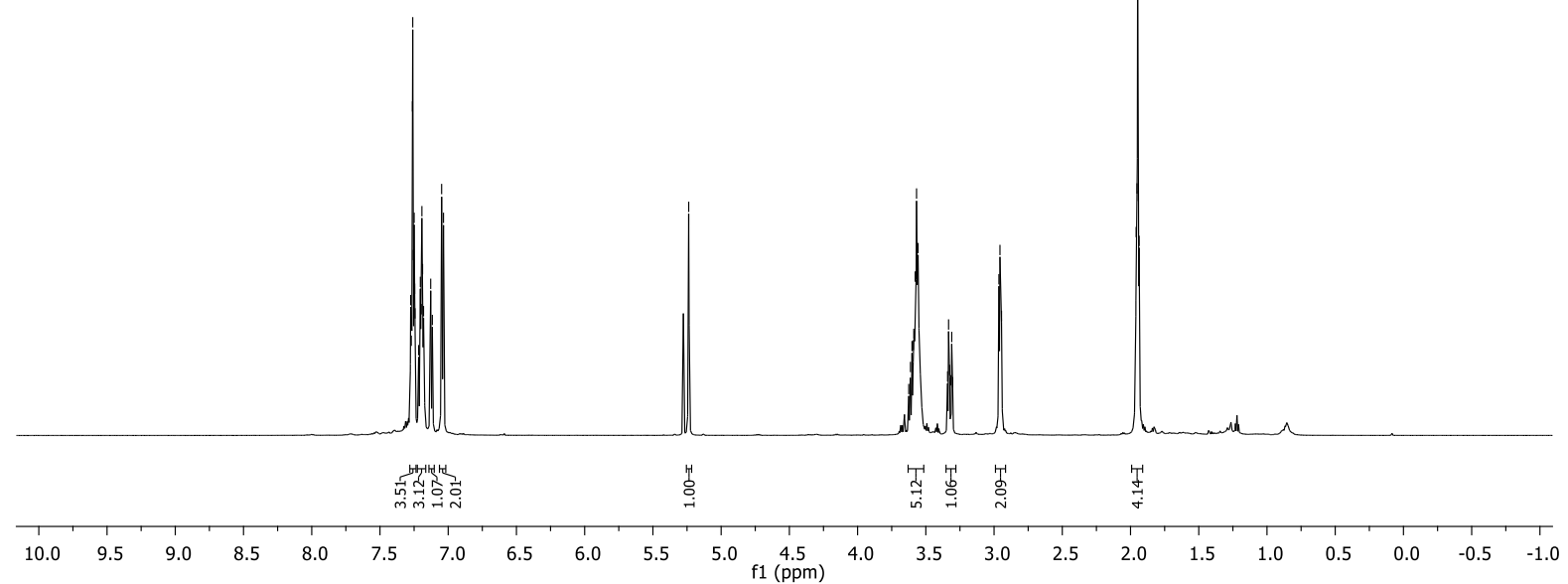


${ }^{13} \mathrm{C}$ NMR $\left(151 \mathrm{MHz}, \mathrm{CDCl}_{3}\right)$

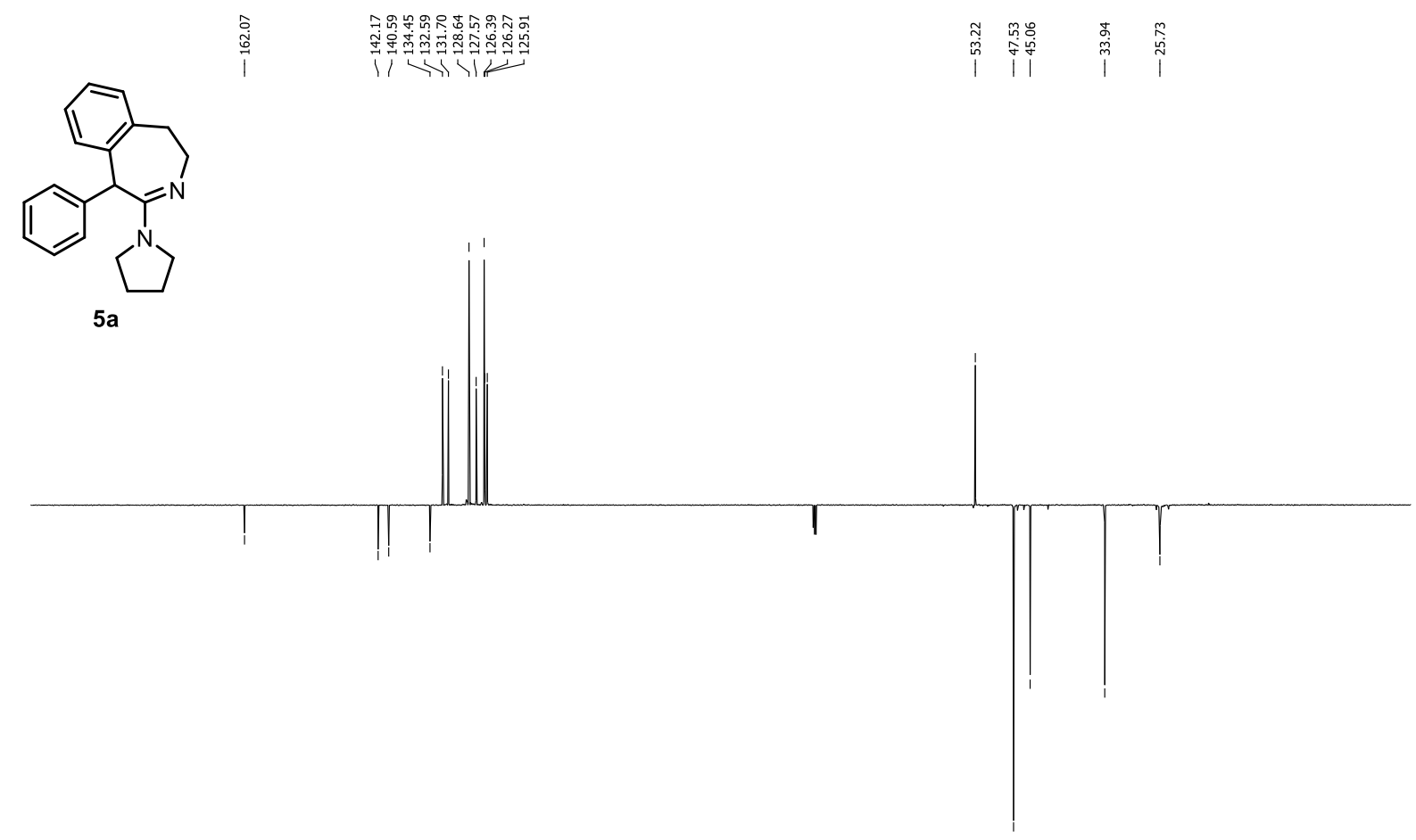

$\begin{array}{lllllllllllllllllllll}190 & 180 & 170 & 160 & 150 & 140 & 130 & 120 & 110 & 100 & 90 & 80 & 70 & 60 & 50 & 40 & 30 & 20 & 10 & 0 & -10\end{array}$

${ }^{1} \mathrm{H}$ NMR $\left(600 \mathrm{MHz}, \mathrm{CDCl}_{3}\right)$

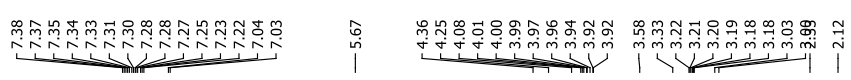

$\underbrace{}_{L^{N}>N^{+}}$

6a
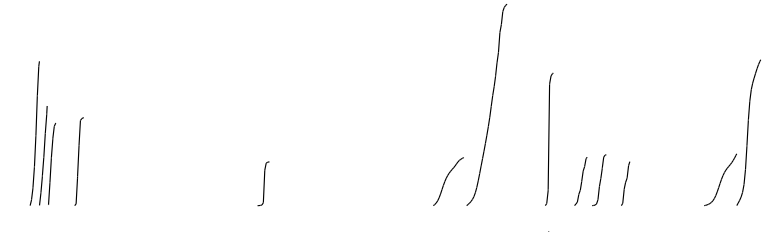

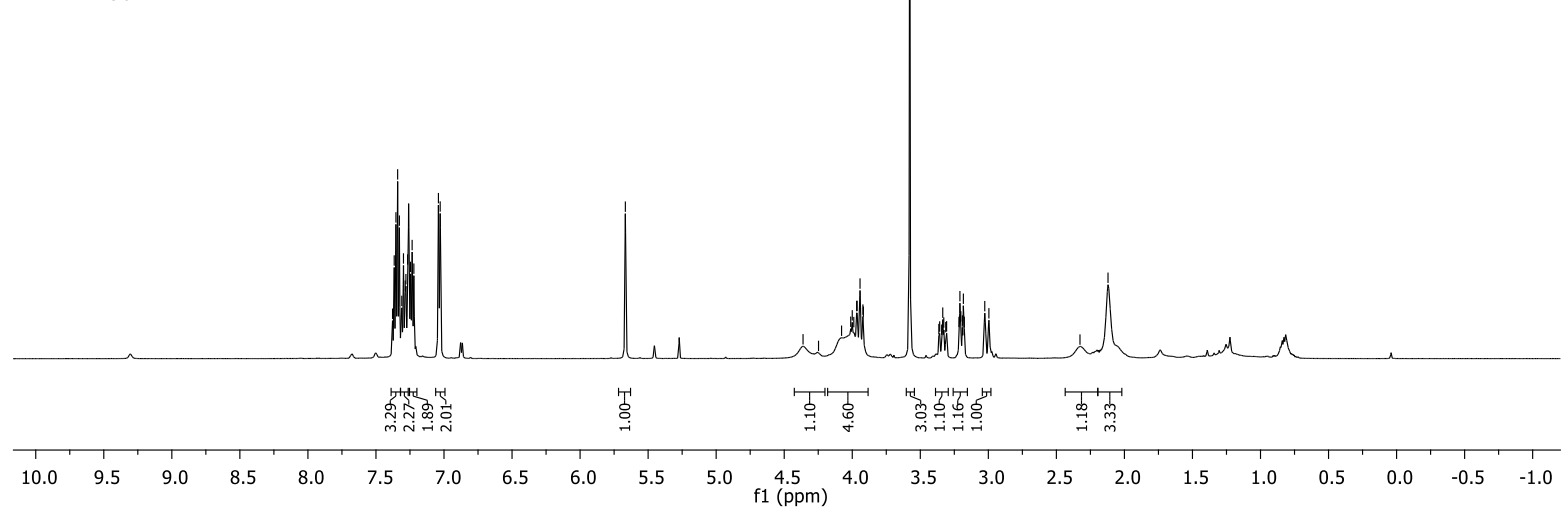


${ }^{13} \mathrm{C}$ NMR $\left(151 \mathrm{MHz}, \mathrm{CDCl}_{3}\right)$

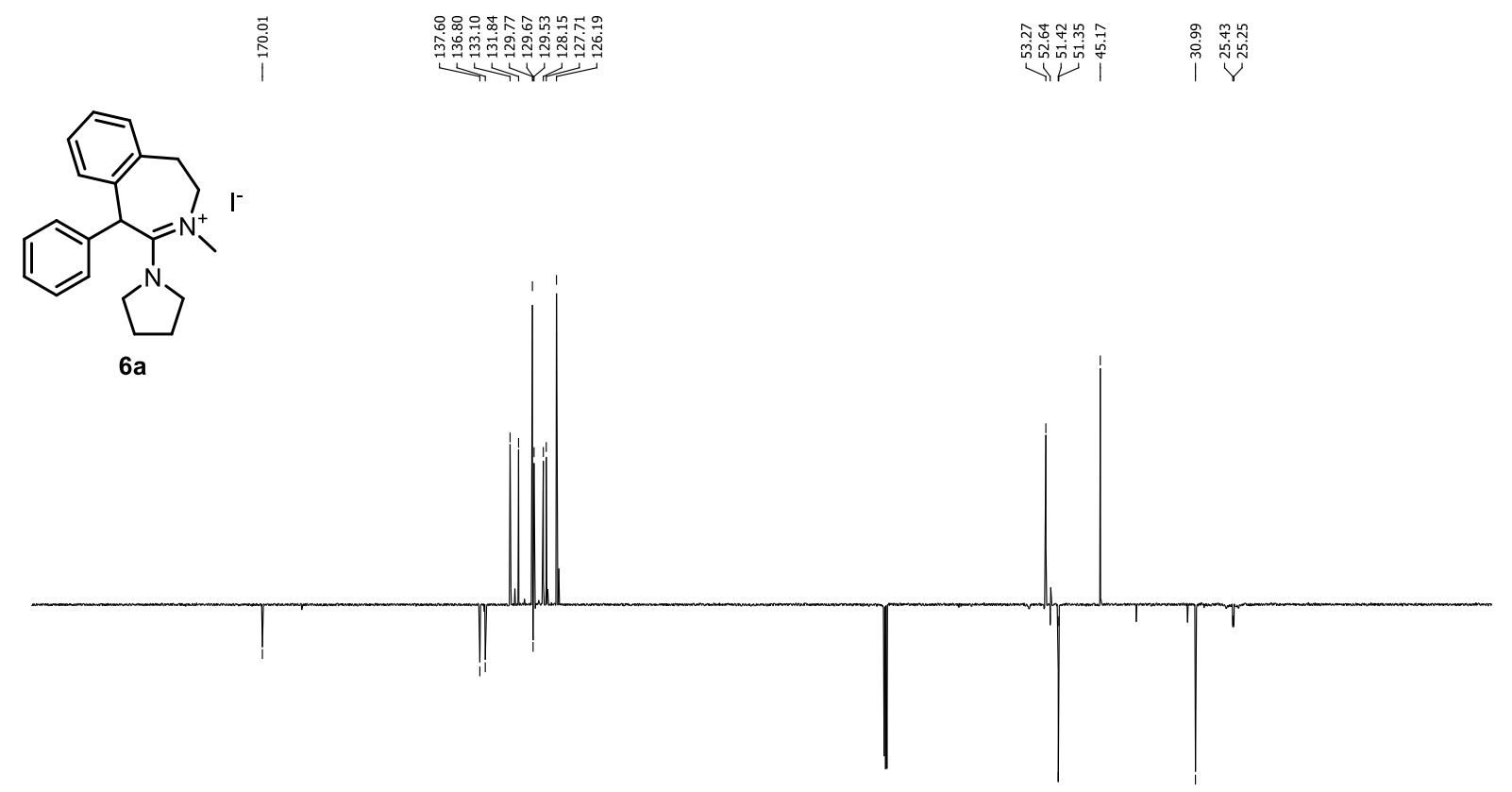

$\begin{array}{lllllllllllllllllllllll}200 & 190 & 180 & 170 & 160 & 150 & 140 & 130 & 120 & 110 & 100 & 100 & 80 & 70 & 60 & 50 & 40 & 30 & 20 & 10 & 0 & -10\end{array}$ 